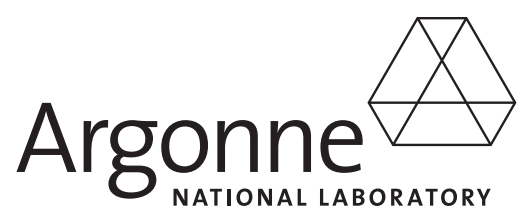

ANL/EVS/AGEM/TR-13-08

\title{
Work Plan for Targeted Investigation at Bendena, Kansas
}

\section{Environmental Science Division}


About Argonne National Laboratory

Argonne is a U.S. Department of Energy laboratory managed by UChicago Argonne, LLC under contract DE-AC02-06CH11357. The Laboratory's main facility is outside Chicago, at 9700 South Cass Avenue, Argonne, Illinois 60439. For information about Argonne and its pioneering science and technology programs, see www.anl.gov.

\section{Availability of This Report}

This report is available, at no cost, at http://www.osti.gov/bridge. It is also available on paper to the U.S. Department of Energy and its contractors, for a processing fee, from:

U.S. Department of Energy

Office of Scientific and Technical Information

P.O. Box 62

Oak Ridge, TN 37831-0062

phone (865) 576-8401

fax (865) 576-5728

reports@adonis.osti.gov

\section{Disclaimer}

This report was prepared as an account of work sponsored by an agency of the United States Government. Reference herein to any specific commercial product, process, or service by trade name, trademark, manufacturer, or otherwise, does not necessarily constitute or imply its endorsement, recommendation, or favoring by the United States Government or any agency thereof. The views and opinions of document authors expressed herein do not necessarily state or reflect those of the United States Government or any agency thereof, Argonne National Laboratory, or UChicago Argonne, LLC. 


\section{Work Plan for Targeted Investigation at Bendena, Kansas}

by

Applied Geosciences and Environmental Management Section Environmental Science Division, Argonne National Laboratory

August 2013

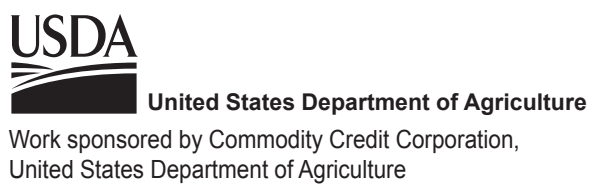




\section{Contents}

Notation.

1 Introduction

2.1 Community Overview................................................................................ 2-1

2.1.1 Water Supply History ………………………........................................ 2-1

2.1.2 History of Grain Storage Operations ....................................................... 2-2

2.1.2.1 Former CCC/USDA Facility ........................................................ 2- 2-3

2.1.2.2 Former Bendena Grain Company Facility ....................................... 2 2-3

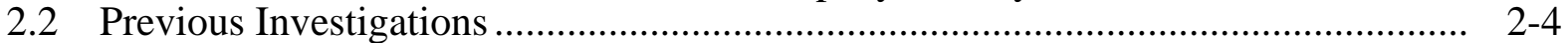

2.2.1 Sampling of Well PWS1 in 1985-1998 ………...................................... 2-5

2.2.2 Preliminary Assessment in 1987 .................................................................. 2-5

2.2.3 Site Inspection in 1987-1988 ............................................................... 2-6

2.2.4 Comprehensive Investigation in 1998 ................................................... 2-7

2.2.5 Sampling of Selected Monitoring Wells in 1999-2005 ................................ 2-8

2.2.6 Supplemental Site Investigation in 2009-2010 .......................................... 2-9

2.2.7 Private Well Sampling in 2013 ………………....................................... 2-10

2.2.8 Additional Investigations Related to Soil and Groundwater

2.3 Geologic and Hydrogeologic Setting.................................................................. 2-12

2.3.1 Regional Geology and Hydrogeology …………......................................... 2-12

2.3.2 Local Setting ................................................................................................ 2-13

2.3.2.1 Site-Specific Geology ................................................................... 2-14

2.3.2.2 Site-Specific Hydrogeology ………………………………........... 2-16

2.3.2.3 Possible External Influences on the Local Groundwater Flow

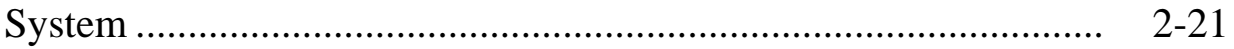

2.3.3 Summary ................................................................................................... 2-22

3 Proposed Technical Program for the Investigation............................................................. 3-1

3.1 Technical Objectives..................................................................................

3.2 Primary Investigation Tasks ……………………............................................. 3-2

3.2.1 Task 1. Conduct Vertical Profiling at the Former CCC/USDA Facility to Determine the Distribution of Carbon Tetrachloride Contamination in Soils and Groundwater.

3.2.2 Task 2. Conduct Vertical Profiling for VOCs Analysis at Selected Locations on the Bendena Ag and Consumer Oil Properties, to Investigate the Vertical and Areal Distribution of Carbon Tetrachloride Contamination and Potential Contaminant Migration Pathways...

3.2.3 Task 3. Determine the Lithologic and Hydrologic Characteristics of the Unconsolidated Units Hosting the Groundwater Flow System

3.2.4 Task 4. Install Permanent Groundwater Monitoring Points to Investigate the Patterns of Groundwater Movement

3.2.5 Task 5. Conduct Groundwater Sampling at the Permanent Monitoring Wells and Nearby Private Wells. 


\section{Contents (Cont.)}

3.3 Investigation Methods.................................................................................. 3-6

3.3.1 Methods for Collecting and Logging Soil Cores ....................................... 3-6

3.3.2 Methods for Sampling and VOCs Analyses of Soils.................................... 3-7

3.3.3 Methods for Groundwater Sampling ……………........................................ 3-. 3-7

3.3.4 Methods for Sampling of the Monitoring and Private Wells.......................... 3-8

3.3.5 Methods for Analysis of Water Samples for VOCs....................................... 3-9

3.3.6 Methods for the Installation of Monitoring Wells ........................................ 3-9

3.3.7 Methods for Handling and Disposal of Investigation-Derived Waste........... 3-9

3.4 Sampling and Reporting Schedule.......................................................................... 3-10

3.5 Quality Assurance and Quality Control.................................................................. 3-11

3.6 Health and Safety ................................................................................. 3-11

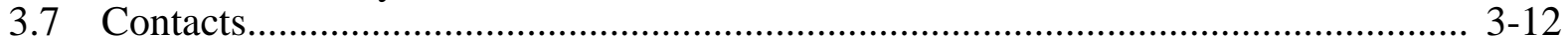

3.7.1 Johnson Farms Contacts …………….......................................................... 3-12

3.7.2 Bendena Ag Contacts............................................................................... 3-12

3.7.3 Consumer Oil Contact............................................................................... 3-13

3.7.4 KDHE Contacts for the Former CCC/USDA Facility ……….......................... 3-13

3.7.5 CCC/USDA Contacts.................................................................................... 3-14

3.7.6 Doniphan County Rural Water District No. 2 Contact ...................................... 3-14

3.7.7 Argonne Contacts.......................................................................................... 3-14

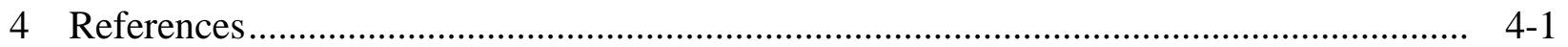

Appendix A: Monitoring Well Registrations...................................................................... A-1

Supplement 1: Titles and Leases for the Former CCC/USDA Facility and Adjacent Properties.

\section{Figures}

1.1 Location of Bendena, Kansas; the former CCC/USDA grain storage facility; and public, domestic, and monitoring wells ........................................................................ 1-4

1.2 Locations of properties of interest in the investigation, showing grain storage structures in 1966

2.1 Locations and current ownership of properties of interest in the investigation, with well PWS1

2.2 Locations of grain storage facilities in 1966 .............................................................. 2-35

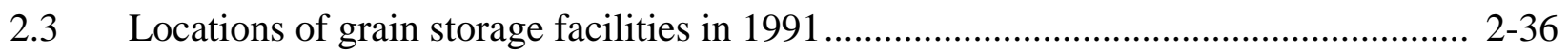

2.4 Sampling locations and results of analyses for carbon tetrachloride in soil gas samples collected during the 1987-1988 KDHE site inspection 


\section{Figures (Cont.)}

2.5 Locations of monitoring wells MW1-MW3 and private wells investigated during the 1987-1988 KDHE site inspection

2.6 Sampling locations during the 1998 KDHE comprehensive investigation

2.7 Shallow soil sampling locations in the 2009-2010 KDHE supplemental site investigation, with carbon tetrachloride results for the Argonne split soil samples.

2.8 Groundwater sampling locations in the 2009-2010 KDHE supplemental site investigation, with carbon tetrachloride results for the KDHE and Argonne split groundwater samples

2.9 Locations of private wells proposed for sampling by the KDHE in 2013.................... 2-42

2.10 Generalized stratigraphic column for the Doniphan County area .............................. 2-43

2.11 Modern topography of the Doniphan County Area ....................................................... 2-44

2.12 West-to-east geologic cross section A-A' across Doniphan County and through Bendena, at the location shown in Figure 2.13 ............................................................. 2-45

2.13 Location of west-to-east geologic cross section A-A' across Doniphan County ........... 2-46

2.14 Local topography of the Bendena area ………............................................................ 2-47

2.15 Locations of hydrogeologic cross sections B-B', C-C', and D-D' at Bendena, with public water supply, domestic, and monitoring wells.

2.16 South-to north hydrogeologic cross section B-B' at Bendena ...................................... 2-49

2.17 West-to-east hydrogeologic cross section C-C' at Bendena …...................................... 2-50

2.18 West-to-east hydrogeologic cross section D-D' at Bendena ....................................... 2-51

2.19 Mechanically contoured potentiometric surface for water levels measured in 1998 in the lower aquifer monitoring wells, including MW7

3.1 Proposed locations for investigative borings 1-11, with locations of existing monitoring wells 


\section{Figures (Cont.)}

3.2 Proposed locations for installation of approximately nine new monitoring wells in the shallow and lower aquifers

3.3 Emergency route from Bendena to the Atchison Hospital in Atchison, Kansas

\section{Tables}

2.1 Analytical results for VOCs in groundwater, 1985-2010

2.2 Analytical results for soil gas samples collected by the KDHE on November 5, 1987

2.3 Construction data for permanent monitoring wells

2.4 Analytical results for VOCs in vertical-profile soil samples collected at location MW4D in March 1998

2.5 Analytical results for VOCs in groundwater samples collected by the KDHE from private wells in February 2013

2.6 Summary of groundwater level measurements for the permanent monitoring wells and well PWS1

3.1 Emergency information for the investigation at Bendena, Kansas................................. 3-15 


\section{Notation}

AGEM Applied Geosciences and Environmental Management

AMSL above mean sea level

BGL below ground level

CCC Commodity Credit Corporation

CPT cone penetrometer

d day(s)

EPA U.S. Environmental Protection Agency

$\mathrm{ft} \quad$ foot (feet)

gal gallon(s)

gpm gallon(s) per minute

hr hour(s)

I.D. inner diameter

in. inch(es)

KDHE Kansas Department of Health and Environment

MCL maximum contaminant level

$\mu \mathrm{g} / \mathrm{kg} \quad$ microgram(s) per kilogram

$\mu \mathrm{g} / \mathrm{L} \quad$ microgram(s) per liter

$\mathrm{mg} / \mathrm{L} \quad$ milligram(s) per liter

mi mile(s)

${ }^{\circ} \mathrm{C} \quad$ degree(s) Celsius

ppbv part(s) per billion by volume

ppm part(s) per million

psi pound(s) per square inch

PVC polyvinyl chloride

RWD 2 Doniphan County Rural Water District No. 2

SDOH Kansas State Department of Health

USDA U.S. Department of Agriculture

VOC volatile organic compound 


\section{Work Plan for Targeted Investigation at Bendena, Kansas}

\section{Introduction}

During the 1950s and 1960s, the Commodity Credit Corporation (CCC), U.S. Department of Agriculture (USDA), operated a grain storage facility in Bendena, Kansas (Figure 1.1). During this time, commercial grain fumigants containing carbon tetrachloride were commonly used by the CCC/USDA and the private grain storage industry to preserve grain in their facilities. In February 1985, carbon tetrachloride was detected in Bendena's public water supply system during routine screening by the Kansas Department of Health and Environment (KDHE) at a level $(10.9 \mu \mathrm{g} / \mathrm{L})$ exceeding the U.S. Environmental Protection Agency (EPA) maximum contaminant level (MCL) and the KDHE risk-based standard of $5.0 \mu \mathrm{g} / \mathrm{L}$ for this contaminant in drinking water (KDHE 1985a,b, 2010b). Subsequent KDHE investigations confirmed the presence of carbon tetrachloride in Bendena's public water supply well (Doniphan County Rural Water District No. 2 well, Figure 1.1) and also detected carbon tetrachloride in soils and groundwater at the former CCC/USDA facility and in groundwater beneath several commercial properties to the south and southeast of the former facility (KDHE 1988, 1998, 2010a).

To determine whether the former CCC/USDA facility represents a source of carbon tetrachloride contamination and its possible relationship to the carbon tetrachloride identified in groundwater, the CCC/USDA has agreed to conduct an investigation at Bendena, in accordance with the Intergovernmental Agreement between the KDHE and the Farm Service Agency of the USDA. The investigation will be performed on behalf of the CCC/USDA by the Environmental Science Division of Argonne National Laboratory, a nonprofit, multidisciplinary research center operated by UChicago Argonne, LLC, for the U.S. Department of Energy.

For this work plan, Argonne compiled historical data related to the CCC/USDA grain storage operations and previous investigations of the carbon tetrachloride contamination at Bendena, as well as other information pertinent to understanding this site. Through a review of all available documents, the KDHE (1988) identified the former CCC/USDA grain storage facility and the grain storage facility formerly operated by the Bendena Grain Co. as potential source areas for the carbon tetrachloride contamination. The former CCC/USDA facility was located on property now owned and operated as a grain storage facility by Johnson Farms, Inc.; the former Bendena Grain Co. facility was located on property that is now owned by Bendena 
Ag., Inc. (Figure 1.2). Previous investigations and the potential source areas are discussed in Section 2.

On the basis of the analyses of the historical data, and in keeping with ongoing discussions with the KDHE and key property owners that affect critical site access, the following specific technical objectives are proposed for the targeted investigation at Bendena:

- Investigate the carbon tetrachloride contamination previously identified in soils and groundwater; evaluate the former CCC/USDA facility (on property now owned by Johnson Farms) as a potential source of contamination to the local groundwater.

- Investigate the distribution of carbon tetrachloride contamination in subsurface soils and groundwater beneath the properties currently owned by Bendena Ag and the Consumer Oil Co., south and southeast of the former CCC/USDA facility.

- Verify the lithologic and hydrologic characteristics of the unconsolidated geologic sequence hosting the groundwater flow system in the vicinity of the former CCC/USDA facility.

- Identify and document the patterns of groundwater flow and potential contaminant migration in the local hydrogeologic system.

- Update the inventory and status of local private wells; conduct limited private well sampling as appropriate, subject to access, to identify potential downgradient receptors.

The detailed scope of work is outlined in Section 3. The results of this study will provide the basis for determining, in consultation with the CCC/USDA and KDHE program managers, what future CCC/USDA actions might be necessary to achieve no further action status for the Bendena site.

Argonne issued a Master Work Plan (Argonne 2002) that has been approved by the KDHE. The Master Work Plan describes the general scope of all investigations at former 
CCC/USDA facilities in Kansas and provides guidance for these investigations. In addition to Section 3.3 of this Work Plan, the Master Work Plan (Argonne 2002) should be consulted for complete details of plans for work associated with the former CCC/USDA facility at Bendena. 


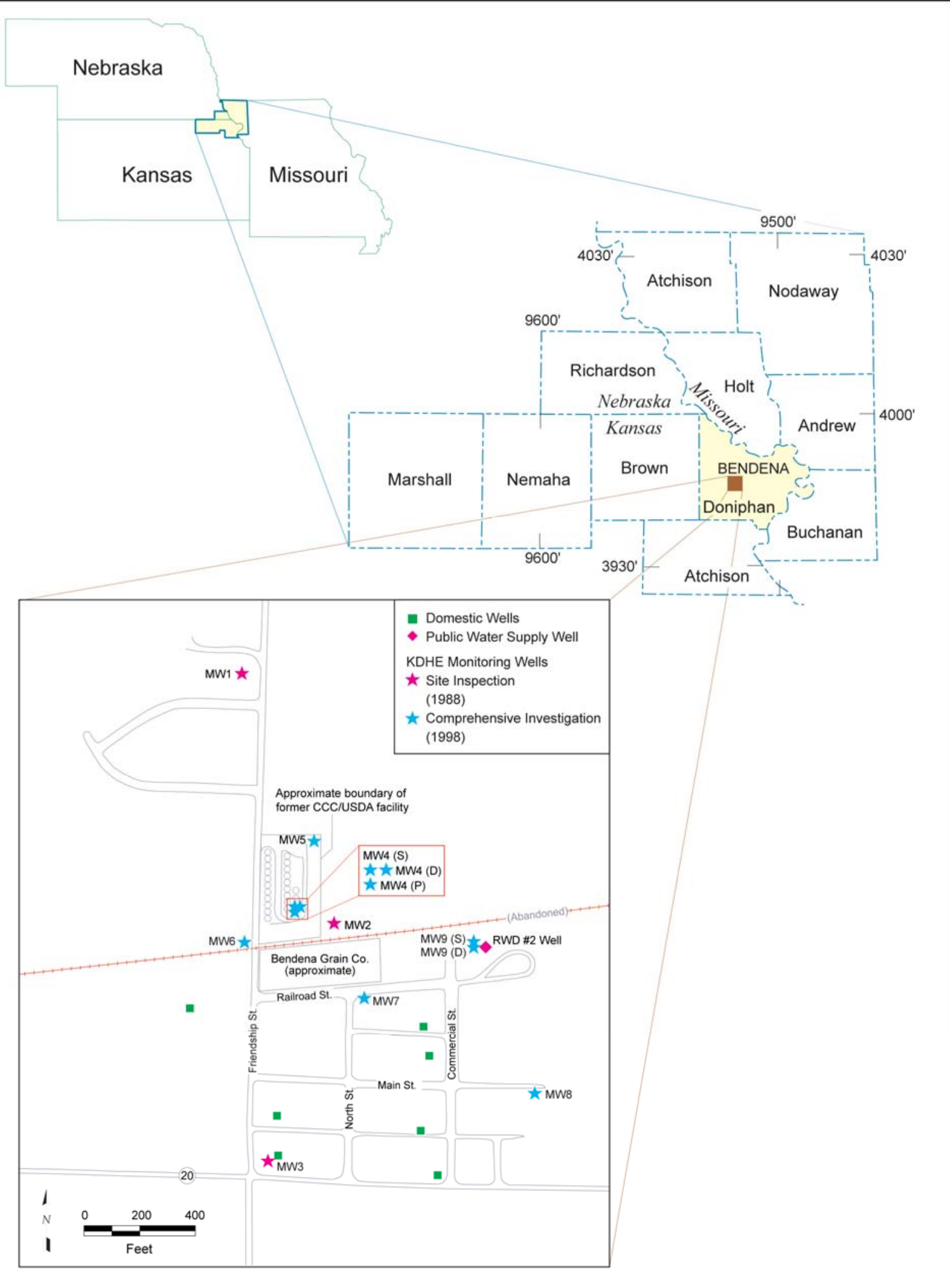

FIGURE 1.1 Location of Bendena, Kansas; the former CCC/USDA grain storage facility; and public, domestic, and monitoring wells. 


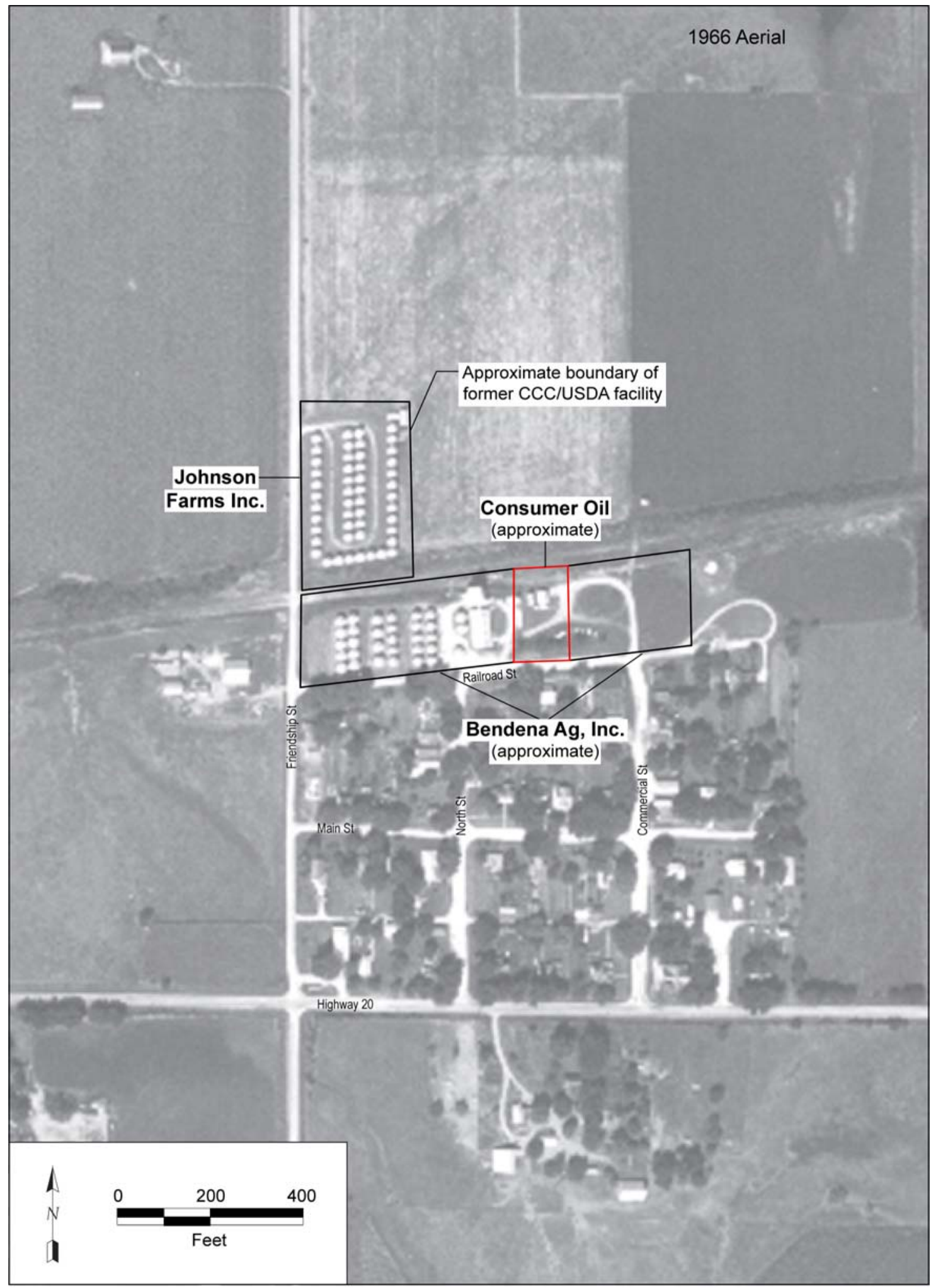

FIGURE 1.2 Locations of properties of interest in the investigation, showing grain storage structures in 1966. Source of photograph: USDA (1966). 


\section{Background and Previous Studies}

\subsection{Community Overview}

Bendena, Kansas, founded in 1877, is an unincorporated rural town of approximately 117 persons (2010 Census) in northeastern Kansas (Independence Township, Section 33, Township 3 South, Range 20 East, Doniphan County), approximately 11 mi north of Atchison, Kansas, and $22 \mathrm{mi}$ west of St. Joseph, Missouri (Figure 1.1). Businesses in the town include Bendena Ag, Inc.; the Consumer Oil Co.; Urban Construction, Inc.; and several others. The community also has a small grocery store, a bank, a post office, and a church. Sometime after the mid 1960s, a small housing development was constructed northwest of the original town. Since then, several individual newer homes have been constructed in the area. Historically, the Chicago and Rock Island Pacific Railroad served the community, but service has been discontinued, and the tracks have been removed.

Bendena is presently served by the Doniphan County Rural Water District No. 2 (RWD 2) system, which obtains its water supply from the Doniphan County RWD 5 and Atchison County RWD 5 systems. The town does not have a sanitary sewer system; all of the homes and businesses have individual septic systems.

\subsubsection{Water Supply History}

Prior to 1964, Bendena residents obtained water exclusively from private wells. In 19621963, the Doniphan County RWD 2 was formed to provide a public water supply by reconditioning a former railroad well near the northeast corner of the town for use as a municipal well (PWS1; Figure 2.1). Well PWS1 is a hand-dug, brick-walled well approximately $23 \mathrm{ft}$ in diameter at the surface. The well decreases in size with depth and is approximately $73 \mathrm{ft}$ deep. Well PWS1 was approved for use as a public water source by the Kansas State Department of Health in November 1964 (SDOH 1964).

As early as the mid 1970s, high concentrations of nitrate were detected in well PWS1. The average nitrate concentration from 1970 to 1983 was $36 \mathrm{mg} / \mathrm{L}$ (equivalent to parts per million, ppm), exceeding the EPA MCL of $10 \mathrm{mg} / \mathrm{L}$ for this substance in drinking water (KDHE 1988). In February 1985, carbon tetrachloride was also detected (at $10.9 \mu \mathrm{g} / \mathrm{L}$ ) in a sample from 
the municipal distribution system (Table 2.1). Because carbon tetrachloride is a suspected carcinogen, the KDHE initiated monitoring of the RWD 2 supply for volatile organic compounds (VOCs).

Shortly thereafter, the KDHE mandated changes to the RWD 2 supply system to bring the concentrations of nitrate and carbon tetrachloride in the water delivered to consumers into compliance with state drinking water standards (KDHE 1985a,b, 1986a,b). The actions taken included connection of the RWD 2 distribution system to the Doniphan County RWD 5 system to permit blending of the waters from well PWS1 and RWD 5 to achieve lower nitrate concentrations in the mixed waters, as well as the installation of an air stripping unit to treat the discharge from well PWS1 for carbon tetrachloride. These modifications were completed in 1988-1989 (KDHE 2010a).

Through the early 1990s, nitrate concentrations continued to rise in well PWS1. Consequently, the fraction of water pumped from well PWS1 and blended with water from RWD 5 progressively decreased. By 1992, blending of the PWS1 and RWD 5 supplies had become economically impractical, and in late 1993 or early 1994, RWD 2 stopped using well PWS1 and began purchasing water exclusively from Doniphan County RWD 5 (KDHE 2010a).

After the use of PWS1 was terminated, RWD 2 continued to purchase water from Doniphan County RWD 5. RWD 2 has also augmented its municipal supply via a connection to the Atchison County RWD 5, to increase the reliability of its supply and to improve delivery pressures in portions of the distribution system (Godfrey 2013).

\subsubsection{History of Grain Storage Operations}

Reviews of historical property documents, aerial photographs, and reports of interviews with individuals associated with the Bendena site indicate that grain storage operations were historically conducted on two adjacent parcels of land in the northwestern portion of the town during the period (prior to 1985) when grain fumigants containing carbon tetrachloride were in common use by the CCC/USDA and the private grain storage industry. These operations were run by the CCC/USDA and the Bendena Grain Company. 


\subsubsection{Former CCC/USDA Facility}

Property and lease documents on file at the office of the Doniphan County Registrar of Deeds (included in Supplement 1, on the compact disc [CD] inside the back cover of this report) indicate that from May 1, 1954, until October 13, 1973, the CCC/USDA leased a parcel of land, approximately 1.5 acres, for a grain storage facility on the east side of Friendship Road and immediately north of the former railroad right-of-way. The property was originally leased from owner W.C. Albers and subsequently from the estate of Mr. Albers and then his son, Herbert Albers. After the termination of the CCC/USDA operations in 1973, the property remained in the ownership of Herbert Albers until September 1986, when it was conveyed to Paul and (wife) Mary Linn Johnson. In February 1993, ownership of the property was transferred to Johnson Farms, Inc., a Kansas corporation owned by Paul and Mary Johnson.

The detailed history of the grain storage activities on this property is not known; however, an aerial photograph shows that 49 round grain bins were located at the facility in 1966, during its occupation by the CCC/USDA (Figure 2.2). A later aerial photograph shows that 20 of the original bins and several larger, newer bins were present at the site in 1991 (Figure 2.3). At present, 18 of the original bins, 2 slightly smaller bins, and 7 larger bins are located on the property, which continues to be used by Johnson Farms for grain storage (Figure 2.1).

\subsubsection{Former Bendena Grain Company Facility}

Figures 2.2 indicates that 29 round grain bins, a grain elevator, a warehouse building, and several other structures were located on property immediately to the south and southeast of the former CCC/USDA facility in 1966. Property records indicate that prior to September 1981, the facilities in this area were located on right-of-way property owned by the railroad, and hence no lease or other legal documents pertaining to its occupancy are on record. Grain storage operations had been conducted on this property since the early 1900s by the Bendena Grain Company (KDHE 1988). Edwin Dennis Ford purchased half of the site facilities in 1968 and the other half in 1981 from the previous owners, Ben and Doris Ainley, who are deceased. The Bendena Grain Company, Inc., was established as a Kansas corporation by E.D. and Carolyn Ford in July 1980. In September 1981 the Fords obtained ownership of the parcel occupied by the grain storage facility, and in 1984 they acquired a second parcel to the east on the former 
railroad right-of-way. The intervening parcel was obtained by the Consumer Oil Co. in November 1981 (Supplement 1).

In December 2004 the Bendena Grain Company was dissolved as a corporation. The parcels formerly owned by the Fords are now owned by Bendena Ag, Inc., and occupied by Bendena Ag and Urban Construction, Inc. Bendena Ag is owned by Patrick Urban.

Figure 2.3 shows that 25 round grain bins, the elevator, the warehouse building, and numerous other structures remained on the Bendena Grain property in 1991. None of the original round grain bins remain presently; however, the elevator and warehouse building are still present and in use at the site (Figure 2.1).

Efforts are continuing to augment the limited documentation available to the CCC/USDA and refine the representation of the Bendena Ag property boundaries in this Work Plan.

\subsection{Previous Investigations}

To date, the carbon tetrachloride contamination at Bendena has been addressed by the following KDHE investigations:

- 1985-1998: Sampling of well PWS1

- 1987: Preliminary assessment

- 1987-1988: Site inspection

- 1998: Comprehensive investigation

- 1999-2005: Sampling of selected monitoring wells

- 2009-2010: Supplemental site investigation

- 2013: Private well sampling 
These prior investigations are discussed below.

\subsubsection{Sampling of Well PWS1 in 1985-1998}

On February 26, 1985, well PWS1 was sampled as part of a statewide investigation to determine the extent of VOCs contamination in public water supplies. Carbon tetrachloride was detected at $10.9 \mu \mathrm{g} / \mathrm{L}$ (KDHE 1987). Follow-up KDHE sampling of well PWS1 on March 11 and March 22, 1985, confirmed the initial analysis, with carbon tetrachloride concentrations of $10.9 \mu \mathrm{g} / \mathrm{L}$ to $14.0 \mu \mathrm{g} / \mathrm{L}$ being identified in samples from the well and the RWD 2 distribution system (Table 2.1).

Sampling of well PWS1 and the RWD 2 distribution system for VOCs analysis was conducted periodically from 1985 to 1989, during which time the KDHE directed RWD 2 to notify its customers of the carbon tetrachloride contamination and take actions to develop a new source of water free from any contamination (KDHE 1985a,b, 1986a,b). Carbon tetrachloride levels ranging from $6.4 \mu \mathrm{g} / \mathrm{L}$ to $23.8 \mu \mathrm{g} / \mathrm{L}$ were reported for PWS1 during this period.

In 1989, an air stripping unit was installed to treat the carbon tetrachloride contamination. The available records show that after this installation, well PWS1 was sampled only sporadically for VOCs analysis. The reported concentrations of carbon tetrachloride detected at PWS1 from September 1991 to June 1998 (the last reported sampling event) ranged from < $0.7 \mu \mathrm{g} / \mathrm{L}$ in September 1996 to $20.5 \mu \mathrm{g} / \mathrm{L}$ in June 1998 (Table 2.1).

\subsubsection{Preliminary Assessment in 1987}

Under a Multi-Site Cooperative Agreement with the EPA Region VII, in October 1987 the KDHE conducted a pre-National Priorities List preliminary assessment of the Bendena groundwater contamination site. This assessment included a physical site inspection, a review of local property records, and a compilation of analytical data from previous sampling of the Bendena public water supply (KDHE 1987). A soil gas investigation initiated during the time frame of the preliminary assessment report was documented as part of the subsequent site inspection (KDHE 1988). 


\subsubsection{Site Inspection in 1987-1988}

The KDHE completed a site inspection for the Bendena site in September 1988. This study included reviews of local property records and documents, interviews with local residents and public officials, a soil gas survey, sampling of 18 private wells and well PWS1 for VOCs analysis, and the installation and sampling of 3 monitoring wells for VOCs analysis (KDHE 1988). The locations investigated during this study are shown in Figures 2.4 and 2.5.

Soil gas samples were collected at depths of 3-6 ft below ground level (BGL) at 12 locations, to aid in the identification of potential sources of the carbon tetrachloride contamination in groundwater (Figure 2.4). The sampling points were distributed on and near the former CCC/USDA and Bendena Grain Co. grain storage facilities (now the Johnson Farms and Bendena Ag properties, respectively; SG1-SG5), near contaminated well PWS1 (SG6-SG7), and at several other locations throughout the town (SG8-SG12; Figure 2.4) The soil gas samples were analyzed for carbon tetrachloride, 1,1,1-trichloroethane, trichloroethylene, toluene, and total hydrocarbons.

Trace to low levels of carbon tetrachloride, 1,1,1-trichloroethane, and trichloroethylene were detected in the soil gas at all of the sampled locations, with the highest concentrations of carbon tetrachloride occurring near the northern edge of the Bendena Grain property and at the southeast corner of the former CCC/USDA facility (at SG4 and SG3; Table 2.2 and Figure 2.4). The KDHE concluded that no distinct contaminant plume could be identified on the basis of these data, noting that underlying clays might act as a barrier to the upward migration of contaminant vapors (KDHE 1988).

Monitoring wells MW1-MW3 were installed during the site inspection, at completion depths ranging from $71.4 \mathrm{ft}$ to $99.6 \mathrm{ft}$ BGL (Figure 2.5 and Table 2.3). Although the wells were constructed with screened intervals of 10-15 ft, each well was equipped with a continuous gravel pack extending to within 25-26 ft of the ground surface. No carbon tetrachloride was detected at well MW1 or MW3; carbon tetrachloride was identified at a concentration of $62.9 \mu \mathrm{g} / \mathrm{L}$ at MW2 (Table 2.1).

Carbon tetrachloride levels identified in well PWS1 during the site inspection ranged from $13.3 \mu \mathrm{g} / \mathrm{L}$ to $20.3 \mu \mathrm{g} / \mathrm{L}$ (Table 2.1 ). 
The 17 private wells sampled for VOCs in this investigation included 12 wells located outside the immediate physical limits of the town (reported as being in use for domestic or livestock purposes), plus 1 domestic well, 1 lawn and garden well, and 3 wells reported as not in use in the town (Map 6 in KDHE 1988). The approximate locations of these wells are illustrated in Figure 2.5. With only one exception, no carbon tetrachloride was identified in the private wells (Table 2.1). The exception was concentrations of 2.6-2.9 $\mu \mathrm{g} / \mathrm{L}$ detected in the Ron Albers domestic well, roughly $0.5 \mathrm{mi}$ west of Bendena (Figure 2.5). The KDHE reported that a line from the Bendena public water supply had recently been extended to the Albers residence at the time of the site inspection. No apparent link was suggested between the carbon tetrachloride contamination identified at the Albers well and at wells MW2 and PWS1 in the town (KDHE 1988).

\subsubsection{Comprehensive Investigation in 1998}

On behalf of the KDHE, in $1998 \mathrm{BE} \& \mathrm{~K} /$ Terranext conducted a comprehensive investigation at Bendena (KDHE 1998). Activities in this investigation included continuous coring of 6 deep borings to determine the local lithologic sequence and depth(s) to bedrock, the installation of 9 new monitoring wells, sampling of subsurface soils for VOCs analysis at one deep boring location, and sampling of groundwater for VOCs analysis (and nitrate analysis; see Section 2.2.8) at 12 previously existing and new monitoring wells, PWS1, and 4 private wells in the town. The locations investigated in the comprehensive investigation are illustrated in Figure 2.6.

At deep boring location MW4, in the south-central portion of the former CCC/USDA facility (Figure 2.6), 14 soil samples for VOCs analysis were collected at intervals of $4-5 \mathrm{ft}$ to a maximum depth of $67 \mathrm{ft} \mathrm{BGL}$. The results of these analyses (Table 2.4) indicate that quantifiable levels of carbon tetrachloride were detected in 4 samples, at depths ranging from $25 \mathrm{ft}$ to $67 \mathrm{ft}$ BGL and concentrations ranging from $3.2 \mu \mathrm{g} / \mathrm{kg}$ to $100 \mu \mathrm{g} / \mathrm{kg}$. Only one soil sample, collected at 50-52 ft BGL, had a reported carbon tetrachloride concentration $(100 \mu \mathrm{g} / \mathrm{kg})$ that exceeds the current KDHE risk-based standard for this contaminant in soils $(73.4 \mu \mathrm{g} / \mathrm{kg})$.

Nine new monitoring wells (MW4P,S,D, MW5-MW8, MW9S,D; Figure 2.6 and Table 2.3) were installed to intersect 3 potential aquifer units (perched, shallow, and lower) that had been recognized by the KDHE during the comprehensive investigation. The lithologic and hydrogeologic information obtained from these borings is discussed in Section 2.3. Groundwater 
samples for VOCs analysis were collected from the new wells soon after each was installed and developed in March 1998 and again in April 1998, approximately 30 days after the final new well was completed. Groundwater samples for VOCs analysis were also collected as part of this investigation from previously installed KDHE monitoring wells MW1-MW3 in November 1997 and April 1998, as well as from well PWS1 in April 1998 (Figure 2.6). The results of the groundwater analyses are in Table 2.1.

Carbon tetrachloride was detected in groundwater at only three locations during the comprehensive investigation (Figure 2.6 and Table 2.1). At MW4 (at the former CCC/USDA facility), carbon tetrachloride was identified at concentrations of 2.6-18 $\mu \mathrm{g} / \mathrm{L}$ at a depth of 20-30 ft BGL (MW4P) and 210-410 $\mu \mathrm{g} / \mathrm{L}$ at a depth of 60-80 ft BGL (MW4S). The value of $410 \mu \mathrm{g} / \mathrm{L}$ for MW4S in March 1998 represents the highest concentration of carbon tetrachloride identified to date in groundwater at the Bendena site. No carbon tetrachloride was detected in deeper groundwater samples collected at this location (111-126 ft BGL; MW4D). Carbon tetrachloride was also identified in groundwater from monitoring well MW2 (75-120 $\mu \mathrm{g} / \mathrm{L}$; sandpacked from $25 \mathrm{ft}$ to $99.6 \mathrm{ft} \mathrm{BGL}$ ), as well as at trace levels (2.2-2.5 $\mu \mathrm{g} / \mathrm{L}$; at $45-65 \mathrm{ft} \mathrm{BGL})$ in well MW9S, near well PWS1. No carbon tetrachloride was detected in groundwater sampled from four private wells (Holzhey, Johnson, Dickson, Phillips) and PWS1 during the comprehensive investigation (Figure 2.6 and Table 2.1).

\subsubsection{Sampling of Selected Monitoring Wells in 1999-2005}

The limited available data indicate that from 1999 to 2005, selected monitoring wells (MW2, MW4P,S,D, MW5, MW9S,D; locations in Figure 2.6) were sampled periodically by the KDHE for carbon tetrachloride analysis, in conjunction with sampling of the groundwater for nitrate analysis (Section 2.2.8). The available information indicates that groundwater samples for VOCs analysis were also collected from wells MW4P,S,D as part of a June 2009 nitrate sampling event (KDHE 2010a). The results of the VOCs analyses are summarized in Table 2.1.

With one exception, the occurrences of carbon tetrachloride identified from the analyses in 1999-2005 are generally consistent with those observed during the 1998 comprehensive investigation (Section 2.2.4). In June 2003, carbon tetrachloride was identified at $83 \mu \mathrm{g} / \mathrm{L}$ in well MW4D (Table 2.1); this detection is not corroborated by any other VOC analyses reported for this well to date. 


\subsubsection{Supplemental Site Investigation in 2009-2010}

In late December 2009 to early January 2010, the KDHE conducted a supplemental site investigation to document the distribution of nitrate (see Section 2.2.8) and carbon tetrachloride contamination in the soils and groundwater (KDHE 2010a). The study was performed by using direct-push techniques to recover samples of groundwater (for analyses of nitrate, ammonia, and VOCs) and soils (for nitrate and ammonia analyses) at selected locations on the Johnson Farms (former CCC/USDA facility), Bendena Ag, and Consumer Oil properties. With the consent of the KDHE and the property owners and on behalf of the CCC/USDA, Argonne obtained split samples for VOCs analyses of both the soil and groundwater samples collected by the KDHE. The locations investigated during the supplemental site investigation are illustrated in Figures 2.7 and 2.8.

Shallow soil cores were collected by the KDHE to a depth of $5 \mathrm{ft}$ BGL at 4 locations at the former CCC/USDA facility (SS01, SS02, SS11, SS12), 2 locations on the Consumer Oil property (SS25, SS26), and 20 locations on the Bendena Ag property (SS03-SS10, SS13-SS24; Figure 2.7). Samples from these cores were obtained by Argonne (from $5 \mathrm{ft} \mathrm{BGL)} \mathrm{for} \mathrm{the} \mathrm{VOCs}$ analyses. No carbon tetrachloride was detected in any of the soil samples analyzed by Argonne. The KDHE did not analyze the soil samples for VOCs.

The KDHE was able to recover groundwater at 9 out of 10 locations targeted for groundwater sampling (GW01 through GW10; Figure 2.8). At each of these locations, discrete sampling from 3 depth intervals (corresponding to the inferred perched, shallow, and lower aquifer units; see Sections 2.2.4 and 2.3) was originally planned. Depths required to penetrate the lower aquifer (near the top of bedrock) could not be achieved, however, with the available directpush equipment, and perched groundwater was recovered at only 1 (GW05P) of 4 locations initially attempted (GW03, GW05, GW08, and GW09; Figure 2.8). The field sampling plan was therefore altered to focus on retrieving groundwater from the inferred shallow aquifer, at the depth of refusal (from 57-79 ft BGL) of the direct-push rods at each sampling location (KDHE 2010a). The results of analyses of the groundwater samples for VOCs reported by the KDHE, with the corresponding values for split samples analyzed by Argonne, are in Figure 2.8 and Table 2.1.

Carbon tetrachloride was identified in the groundwater at 7 of the 9 sampled locations, and at 6 locations at concentrations greater than the KDHE risk-based standard and the EPA's 
MCL of $5.0 \mu \mathrm{g} / \mathrm{L}$ for this contaminant. The highest concentrations of carbon tetrachloride reported by both the KDHE and Argonne laboratories were detected at locations GW02 $(190-240 \mu \mathrm{g} / \mathrm{L}$, at $69.5-74.5 \mathrm{ft}$ BGL) and GW07 (190-244 $\mu \mathrm{g} / \mathrm{L}$, at $63-67 \mathrm{ft}$ BGL), at the southeast corner of the former CCC/USDA facility (now Johnson Farms) and the eastern edge of the western Bendena Ag property, respectively (Figure 2.8).

\subsubsection{Private Well Sampling in $\mathbf{2 0 1 3}$}

In coordination with the CCC/USDA, in 2012 the KDHE developed a work plan for groundwater sampling for VOCs analysis at the locations of six former CCC/USDA facilities in Kansas identified in the Intergovernmental Agreement between the KDHE and the USDA, including the former Bendena facility. On the basis of previous sampling activities performed at the site and a KDHE review of recent state well completion records, the KDHE identified 18 private wells within roughly $1 \mathrm{mi}$ of Bendena for potential sampling; the locations of wells as identified in the KDHE work plan are shown in Figure 2.9 (KDHE 2012).

To date, Argonne has not received a detailed report of the private well sampling event. Data provided by the CCC/USDA indicate, however, that 8 private wells in the Bendena area were sampled by the KDHE in February 2013 (Table 2.5; CCC/USDA 2013). The well owners named in association with several of the sampled wells either do not correspond with those identified in the KDHE work plan or are incomplete (Figure 2.9 and KDHE 2012). The available results indicate, however, that carbon tetrachloride was detected in only one of the tested wells. A carbon tetrachloride concentration of $4.4 \mu \mathrm{g} / \mathrm{L}$ is reported for the "Albers" well; this observation is consistent with the low level of carbon tetrachloride detected in the Ron Albers domestic well during the $1988 \mathrm{KDHE}$ site inspection (Section 2.2.3).

\subsubsection{Additional Investigations Related to Soil and Groundwater Contamination}

As noted in Section 2.1.1, concentrations of nitrate exceeding the EPA's MCL of $10 \mathrm{mg} / \mathrm{L}$ for this contaminant in groundwater were initially identified at well PWS1 in the early 1970s, and increasing levels of this substance led to the discontinuation of use of PWS1 as a public water supply well in the early 1990s. Elevated nitrate concentrations were also historically identified in a number of the private wells and several monitoring wells sampled by the KDHE 
during the 1988 site inspection and 1998 comprehensive investigation (Sections 2.2.3 and 2.2.4; KDHE 1988, 1998).

The nitrate contamination identified at Bendena is not linked to past operations at the former CCC/USDA grain storage facility.

Limited data available to Argonne indicate that, from 2001 to 2005, selected monitoring wells were sampled for nitrate (and carbon tetrachloride) analysis (KDHE 2010a). In 2005, the KDHE recommended that the area affected by groundwater contamination be divided administratively into two "sites" to facilitate investigation of the carbon tetrachloride and nitrate contamination issues separately, with the nitrate contamination being addressed under the KDHE's State Water Plan. At that time, the nitrate component was added into the State Water Plan's long-term monitoring program. Available documentation shows that periodic sampling of selected monitoring wells and well PWS1 by the KDHE for nitrate analysis occurred through at least 2009 (KDHE 2010a).

In December 2009 and January 2010, the KDHE performed soil and groundwater sampling as part of the supplemental site investigation (Section 2.1.5) to study the potential distribution of nitrate contamination in these media beneath the Johnson Farms, Bendena Ag, and Consumer Oil properties (KDHE 2010a).

Since the completion of the supplemental site investigation, both Johnson Farms and Bendena Ag have received approval from the KDHE for work plans submitted, and/or investigations conducted, to address nitrate contamination identified on these respective properties under individual agreements with the KDHE as part of the state's Voluntary Cleanup and Property Redevelopment Program (Larsen 2012a,b; GSI 2011, 2012).

The CCC/USDA recently became aware of the installation of a series of shallow groundwater monitoring/observation wells in association with an investigation of leaking storage tanks on the Consumer Oil property. No further information is available at this time, and well registrations have not yet been posted on the online Kansas Geological Survey Water Well Completion Records Database.

Although the nitrate studies do not directly address the identified carbon tetrachloride contamination, limited information pertaining to the local geologic and hydrogeologic setting 
provided in several of these reports is incorporated, as appropriate, into the discussions in Section 2.3.

\subsection{Geologic and Hydrogeologic Setting}

\subsubsection{Regional Geology and Hydrogeology}

Bendena lies in the Dissected Till Plains region of the Central Lowlands Province of the Missouri River Basin. In this area, unconsolidated glacial drift materials - primarily finegrained till, with variably abundant lenses, stringers, or beds of coarser-grained glacial outwash, fluvial, and lacustrine materials - deposited during several periods of Pleistocene continental glaciation unconformably overlie an eroded bedrock surface composed primarily of interbedded Pennsylvanian age shales, limestones, and minor sandstones (Aber 1988). Since the retreat of the glaciers, these deposits have been modified by subsequent episodes of uplift and headward erosion along downcutting streams, coupled with the deposition of aeolian silts and clays (loess) on upland areas and alluvial sediments in the floodplains along major stream channels. A generalized stratigraphic column for the Doniphan County area is in Figure 2.10.

The modern topography of the Doniphan County area primarily reflects the more recent history of post-glacial erosion and deposition (Figure 2.11). Upland areas are generally smooth to gently rounded, with wide, gently sloping valleys along the major rivers and streams. Along the margins of the uplands, headward erosion along smaller tributary drainageways has resulted in a relatively dissected, hilly to rolling land surface (Dort 1987).

The regional stratigraphic and topographic relationships outlined above are illustrated schematically in geologic cross section A-A' (Figure 2.12), which extends approximately $17 \mathrm{mi}$ from west to east through Bendena (Figure 2.13). Cross section A-A' was constructed by using lithologic data from test borings drilled and logged by the Kansas Geologic Survey, several public supply wells, and boring logs for several monitoring wells installed by the KDHE at the Bendena site. The logs represented in section A-A' are being provided under separate cover.

Figure 2.12 indicates that Bendena — particularly the former CCC/USDA facility — is located on a relative upland, overlying up to140 ft of loess and glacial till materials. Figure 2.12 further demonstrates, however, that the thickness of the unconsolidated deposits can vary 
significantly over relatively short lateral distances, because of the combined influences of topographic downcutting along drainageways and local variations in the elevation of the underlying bedrock surface.

In Doniphan County, groundwater levels have historically been mapped only in the alluvial deposits along principal streams (Bayne 1973). In these deposits, groundwater flow closely follows the surface elevation gradient downstream. Away from the streams, the highest groundwater elevations correspond with the topographically highest upland areas (Bayne 1973). Groundwater movement from these areas is necessarily downgradient to the lower elevations along the streams. A number of local topographically controlled flow systems might occur across the county. Within these flow systems, groundwater flow paths will be influenced by the distribution of higher-permeability, coarser deposits (sands and gravels) in the glacial materials. These flow paths might be locally complex. This is the pattern of groundwater movement mapped in the analogous glacial deposits of adjacent Brown County (Bayne and Schoewe 1967).

In the upland areas, the thickness of saturated materials can exceed $100 \mathrm{ft}$ (Bayne and Schoewe 1967); water yields are low, however, because of the heterogeneous character of the glacial materials. In areas of thicker sands, moderate yields (20-40 gpm) are obtainable, but elsewhere yields from thin sands interbedded with the tills generally yield supplies of less than 10 gpm.

\subsubsection{Local Setting}

Bendena is located on the southeastern flank of a topographic high trending northwestsoutheast that forms a local topographic and drainage divide through the community (Figure 2.14). The location of the former CCC/USDA facility lies along the gently sloping crest of this divide, at an elevation of about 1,125 ft above mean sea level (AMSL). Surface runoff from the area flows predominantly to the southwest and southeast into two small drainageways that merge approximately $0.25 \mathrm{mi}$ south of the town. About $25 \mathrm{ft}$ of topographic relief exists within the town. 


\subsubsection{Site-Specific Geology}

The primary sources of existing site-specific geologic and hydrologic data for this investigation are the reports of the site inspection and comprehensive investigation studies conducted by the KDHE $(1988,1998)$, which include lithologic data for 12 borings advanced by the auger drilling method. Limited additional lithologic information for the shallower portions of the stratigraphic sequence is also provided in reports for investigations of nitrate contamination on the Johnson Farms property (Larsen 2012a,b). The geologic logs for the borings specifically discussed in this section are in the reports of previous investigations (KDHE 1988, 1998; Larsen 2012b).

During the 1988 site inspection, 3 borings drilled to reported depths of approximately 71-99 ft below ground level (BGL; completed as monitoring wells MW1-MW3; Figure 2.15 and Appendix A) did not fully penetrate the unconsolidated sequence to bedrock. Similarly, several borings more recently advanced and geologically logged on the Johnson Farms property (two of which were completed as monitoring wells MW10P and MW10S; Table 2.3, Figure 2.15, and Appendix A) penetrated to maximum depths of 63-65 ft BGL and did not reach bedrock (Larsen 2012a,b).

In the 1998 comprehensive investigation, 6 borings completed as monitoring wells MW4D, MW5-MW8, and MW9D (Table 2.3, Figure 2.15, and Appendix A) were continuously cored to refusal at or near the top of bedrock, at reported depths of 122-140 ft BGL. However, poor core recoveries in the deeper sections of several of these borings and apparent discrepancies in the lithologic descriptions presented in the geologic core logs (KDHE 1998), KDHE well registration documents (Appendix A), and interpretive diagrams for these borings (KDHE 1998) make it difficult to evaluate the vertical sequence of unconsolidated materials at depths greater than approximately 60-90 ft with confidence. Because of these factors, a detailed stratigraphic interpretation of the deeper subsurface cannot be developed on the basis of available data. In general, Argonne has employed the information presented in the geologic core log for each boring as the basis for the discussions that follow, under the assumption that these represent the most reliable descriptions of the lithologies encountered in previous drilling programs.

Figures 2.16-2.18 present three cross sections (B-B', C-C', and D-D'), based on the sources described above, illustrating the generalized stratigraphic sequence in the vicinity of the former CCC/USDA facility. The lithologic logs for borings included in these sections are in 
reports of previous investigations (KDHE 1988, 1998; Larsen 2012b). The locations of the sections are shown in Figure 2.15.

The available geologic information suggests that the unconsolidated section at Bendena is broadly divisible into two stratigraphic units: (1) an upper unit consisting predominantly of clay to silty clay with some thin sand stringers with depth, but lacking significant deposits of coarsergrained materials, and (2) a more heterogeneous lower unit consisting of interbedded clays and silts with variably abundant disseminated gravel and discontinuous lenses, stringers, or beds of sand and gravel.

The available descriptions of the upper unit are relatively consistent from borehole to borehole. This unit is described primarily as brown, red-brown, or mottled gray clay or silty clay. Near the surface the clays are generally described as somewhat plastic, but they might become stiffer with depth. Most of the materials within the first $10 \mathrm{ft}$ BGL are described as brown in color, becoming more gray-brown or mottled brown with depth. Regional data (Figure 2.12) suggest that $10-15 \mathrm{ft}$ of loess is expected overlying a sequence of till at Bendena. Although it is not possible to identify a loess unit clearly from the available log descriptions, the existing information is generally consistent with this interpretation. The lower, generally stiffer portion of the upper unit (at depths of about 15-20 ft or more) might also represent loess or reflect a transition to fine-grained glacial material. An apparently discontinuous layer of caliche or a thin "hard zone" reported in several borings at elevations of approximately 1,060-1,080 ft AMSL might represent a paleosoil horizon. Minor, thin sand beds or partings are occasionally reported near the base of the clay-silt unit.

The available descriptions of the lower unit suggest that this interval is composed of glacial till. Fine-grained intervals in the unit are described as gray to black or occasionally redbrown clay or silt, often containing disseminated gravel. Interbedded lenses of sand and gravel are heterogeneously distributed and vary in character, ranging from fine gray or brown sand to coarse gray sand with gravel. The sands are often described as subangular to subrounded, consisting of quartz with some feldspar and occasional limestone. The coarse-grained deposits vary in thickness from a few inches to several feet or more; in some cases they are stacked to produce sequences several feet thick. Both the vertical sequence and the relative abundance of interbedded fine and coarse-grained materials vary significantly from borehole to borehole; coupled with the limited core recovery in many of the deeper intervals, this variability precludes 
correlation or determination, with confidence, of the thicknesses of individual coarse-grained or fine-grained deposits across the investigation area.

Except for gray shale encountered at a depth of $132.5 \mathrm{ft}$ BGL at MW6 and at $120 \mathrm{ft}$ BGL at MW8, no other investigative borings have recovered samples of the local bedrock. The top of bedrock illustrated in the cross sections has been interpreted primarily on the basis of auger refusal. The inferred bedrock surface might slope gently to the south (Section C-C'; Figure 2.17); this surface and the suggested thickness of the unconsolidated section are consistent with the regional-scale stratigraphy and mapping of the bedrock surface.

\subsubsection{Site-Specific Hydrogeology}

The KDHE measured groundwater levels in monitoring wells MW4P,S,D, MW5-MW8, and MW9S,D on April 13, 1998, prior to purging and sampling of these wells over a three-day period (Appendix E in KDHE 1998). These data (Figures 2.16-2.18) appear to represent the most recent coincident set of water level measurements made in all of the permanent monitoring points available at Bendena at any given time. Monitoring wells MW10P and MW10S (Larsen 2012b) were installed after the April 1998 measurement event. A compilation of reported groundwater level measurements for the monitoring wells is in Table 2.6.

At locations MW4 and MW9 with multiple wells (Figure 2.17), the measured levels on April 13, 1998, and subsequently (Table 2.6) indicate the presence of variable downward (vertical) hydraulic gradients in the flow system. Where monitoring wells are screened in sands and gravels, the indicated levels fall within the overlying, presumably less permeable finegrained materials, suggesting that at least semi-confined conditions exist for the penetrated sand lenses.

In the comprehensive investigation report (KDHE 1998), the water levels measured in April 1998 were interpreted as representing three distinct water-bearing units: (1) a perched aquifer in the upper portion of the upper (clay-silt) unit (identified only in well MW4P), (2) a shallow aquifer near the base of the upper (clay-silt) unit (wells MW4S and MW9S), and (3) a lower aquifer in the lower (till) unit (wells MW4D, MW5-MW8, and MW9D). This terminology and the hydrostratigraphic interpretation were employed in subsequent reports of the KDHE and other investigations; in some cases they have been used as a basis for targeting borings for 
groundwater sampling across the area, in the absence of more location-specific hydrogeologic data (KDHE 2010a; Larsen 2012a,b). In light of the interpretive and practical significance associated with this terminology, each of these units is reviewed in detail below, on the basis of presently available data, to evaluate its apparent hydrogeologic characteristics.

\section{Perched Aquifer}

The perched aquifer was identified by the KDHE from the observation that during drilling at the MW4 location (Figures 2.16 and 2.17), water entered the borehole at a depth of approximately $25 \mathrm{ft}$ BGL (in the clay-silt unit) and flowed downward. No sand was observed at this depth, and the flow of groundwater was attributed to a thin zone in the clay possessing secondary porosity (KDHE 1998).

The perched aquifer was not reported in soil borings MW5-MW9. Similarly, monitoring well MW10P (Figure 2.16) and an investigative boring completed at a depth equivalent to that of MW4P (25 ft BGL), approximately $250 \mathrm{ft}$ north of MW4P at the former CCC/USDA facility, failed to produce sufficient groundwater for sampling from this targeted unit (Larsen 2012a,b). Three direct-push borings targeted in this unit on the Bendena Ag property to the south and southeast of MW4P (locations GW03, GW08, and GW09; Figure 2.8) also failed to produce groundwater in a reasonable time frame for sampling (KDHE 2010a). Several other investigative borings advanced to the perched aquifer at the former CCC/USDA facility successfully recovered groundwater for sampling, but the observed water accumulation rates were very slow (Larsen 2012a). Together, these observations suggest that the perched aquifer as recognized at MW4 might not be widely or uniformly developed, instead representing potentially localized occurrence(s) of slightly more favorable, saturated permeability within the generally fine-grained upper portion of the clay-silt unit.

The characteristics reported for the perched aquifer at MW4 (KDHE 1998) do not necessarily indicate the presence of a true perched aquifer, in the sense that the materials underlying the zone are not saturated with water. Rather, the permeability of the surrounding fine-grained materials might be sufficiently low to preclude the release of groundwater present in the sediments at a rate conducive to direct observation, or to permit groundwater sampling over a relatively short period. This possibility is supported by observations that the fine-grained materials in the shallower part of the clay-silt unit have generally been described as "sticky" and 
“damp” to "moist” or "wet” (KDHE 1998; Larsen 2012a,b), suggesting that they might be watersaturated.

Only two monitoring wells have been completed in the perched aquifer: MW4P and MW10P, at depths of 25-30 ft BGL on the former CCC/USDA facility (now Johnson Farms) property (Figure 2.15). No potential groundwater flow direction can be estimated for this unit, and no coincident measurements of groundwater levels have been obtained from these wells. Well MW10P was reported as “dry” following its installation in July 2012 (Larsen 2012b).

\section{Shallow Aquifer}

Three monitoring wells have been completed in the shallow aquifer (MW4S, MW9S, and MW10S; Figure 2.15), at depths of approximately 65-82 ft BGL (Table 2.3) - near the base of the upper (clay-silt) unit or the top of the lower (till) unit (Figures 2.16 and 2.17). Lithologic logs for the MW4 and MW9 locations (KDHE 1998) indicate predominantly clay to silty clay in the screened intervals at these wells, with a few thin horizons of wet sand. At MW10, only moist to wet silty clay is indicated (Larsen 2012b). Estimates of the hydraulic conductivity of the thin sands penetrated at MW4S and MW9S, calculated on the basis of grain size analyses, ranged from approximately $10 \mathrm{ft} / \mathrm{d}$ to $22 \mathrm{ft} / \mathrm{d}$ (KDHE 1998).

Poor core recovery at the MW4 location has hampered identification of the potential total thickness of sand stringers that might be present in and near the interval screened in MW4S (60-80 ft BGL). Somewhat better core recoveries at the MW9 location (MW9S, screened at 45-65 ft BGL), however, resulted in reports of thin wet intervals occurring periodically throughout the underlying interval, from $65 \mathrm{ft}$ BGL to the depth of auger refusal at $128 \mathrm{ft}$ BGL. This finding provides little clear hydrostratigraphic basis for distinguishing the vertical limits of the shallow aquifer as a unique water-bearing unit. Similarly, the screened interval indicated for monitoring well MW10S (approximately 45-65 ft BGL; Table 2.3) appears to fall at a depth (and elevation) intermediate between the nearby MW4P (perched aquifer) and MW4S (shallow aquifer) screened intervals.

Lithologic logs for the remaining deep borings (MW5-MW8; KDHE 1998) also show various wet intervals associated with silty clays, fractured clays, and thin sands sporadically distributed both above and below the depths (and elevations) screened in the MW4S, MW9S, 
and MW10S wells, clouding the definition of the shallow aquifer. As noted for the perched aquifer, the available data for the shallow aquifer and surrounding sediments suggest that groundwater saturation might be present throughout much of the subsurface at Bendena, though it might be readily evident only in zones possessing relatively greater primary or secondary permeability.

Three monitoring wells (the minimum required for estimation of a linear flow direction) are currently identified as representing the shallow aquifer; however, no concurrent measurements of the static groundwater levels in these wells have been made to date. Further, the recognized presence of vertical hydraulic gradients and lithologic heterogeneity in the hydrostratigraphic sequence suggest that multiple groundwater level monitoring points completed in each potential water-bearing interval of interest might be required to accurately estimate the vertical and lateral direction(s) of groundwater movement through the local flow system at any given location and depth.

\section{Lower Aquifer}

The lower aquifer, as described in the comprehensive investigation report (KDHE 1998), consists predominantly of dense, low-permeability silts interbedded with layers of more permeable silts and sands of variable thicknesses. The inferred vertical extent of the lower aquifer is not clearly defined. Across the site, individual lenses of coarser-grained materials in the till cannot be correlated reliably among boreholes, but they appear to occur variably throughout the vertical extent of the till (and into the lower portion of the overlying clay-silt unit). The presently existing monitoring wells that penetrate the lower aquifer, MW4D, MW5MW8, and MW9D (Figures 2.16-2.18), are generally completed in the lowest portion of the till complex (at depths of approximately 120-140 ft BGL; Table 2.3) but are not screened consistently relative to lithology, elevation within the unit, or position relative to the inferred bedrock surface. Estimates of the hydraulic conductivity of the thin sands penetrated at MW4D and MW9D, calculated on the basis of grain size analyses, ranged from approximately $6 \mathrm{ft} / \mathrm{d}$ to $11 \mathrm{ft} / \mathrm{d}$ (KDHE 1998).

Three monitoring wells installed by the KDHE in 1988, described as penetrating the lower aquifer (MW1-MW3; Figures 2.15-2.17) were completed in the upper to middle portions of the till complex at depths of approximately 71-99 ft BGL (KDHE 1988, 1998). These wells 
were abandoned by the KDHE in 2006 because of the presence of continuous gravel packs that extended to within 25-26 ft of the ground surface (Table 2.3 and Appendix A), thus providing artificial conduits for the potential vertical migration of groundwater and contaminants between the shallower and deeper portions of the local hydrogeologic system. The KDHE reported that the upper portion of the casing in each well was removed, while the remaining lower casing was plugged with pumpable grout; however, the continuous gravel packs could not be removed (KDHE 2010a).

In the comprehensive investigation report, the KDHE (1998) presented an interpretation of the April 1998 groundwater levels measured in wells MW4D, MW5, MW6, MW8, and MW9D as indicating groundwater flow in the lower aquifer to the east-southeast (Figure 7 of the comprehensive investigation report [KDHE 1998]); water levels measured in wells MW1-MW3 were not included in this evaluation, because of the extended gravel packs noted above. In coming to this conclusion, however, the KDHE also rejected the measured water level for monitoring well MW7, under the assumption that this well is completed in a lower hydrogeologic unit than the surrounding monitoring wells. This assumption is not supported, however, by Figure 2.18, which illustrates that the screened intervals in adjacent wells MW6, MW7, and MW8 are located at consistent elevations and positions with respect to the underlying bedrock surface (although variations are evident in the specific lithologies screened in each deep well).

If the water level measurement for well MW7 is included in the 1998 data set for the lower aquifer monitoring wells, the resulting (mechanically contoured) potentiometric surface shown in Figure 2.19 suggests that ambient groundwater flow in the deepest part of the unconsolidated sequence (the lower portion of the till) might be generally toward the south across much of the Bendena investigation area, from a possible local high at the former CCC/USDA (now Johnson Farms) property. This observation suggests an element of topographic control on the local groundwater flow system, as Figure 2.14 indicates a drop in the local land surface elevation, to levels of approximately 1,060 ft AMSL or less, toward the headwaters of the intermittent creek valley directly south of the town. Nevertheless, the presence of the vertical gradients noted above, as well as variations in the relative permeabilities of the heterogeneously distributed lithologies in the till complex, are likely to influence the actual detailed pathways of groundwater (and potential contaminant) migration throughout the groundwater flow system. 


\subsubsection{Possible External Influences on the Local Groundwater Flow System}

As outlined in Section 2.1.1, since the early-mid 1990s the community of Bendena has received its water supply from a public water system (Doniphan County RWD 2) that obtains water from sources exclusively outside the Bendena area. Prior to 1964, however, the residents of the town obtained groundwater for domestic and other purposes from individual private wells. From 1964 to approximately 1994, groundwater was supplied to the RWD 2 system by well PWS1 at the northeastern corner of the town.

No records pertaining to the specific historic use or potential current use (domestic, stock watering, lawn and garden, etc.) of private wells in the immediate vicinity of the former CCC/USDA facility and the town are presently available to Argonne. However, some private wells remained available for sampling by the KDHE in 1988, 1998, and 2013 (KDHE 1988, 1998, 2012; CCC/USDA 2013).

Limited information reported for well PWS1 indicates that, when it was approved for public water supply use in 1964, it was equipped with a submersible pump capable of flowing up to $35 \mathrm{gpm}$ at a working pressure of 40 psi. Reports further indicate that this well initially (in 1964) filled the 38,000-gal water storage standpipe in the town in approximately $11 \mathrm{hr}$, with little observed drawdown in the well (Duncan 1964). In 1988, the KDHE reported the pumping rate of PWS1 (while operating as a municipal supply well) as 16-20 gpm (KDHE 1988). Historically, the town's water usage from well PWS1 before connection of the municipal system to the RWD 5 supply was reported in 1987 as approximately 3 million gallons per year or about 250,000 gal per month (Novak and Lay 1987). In late 1993 or early 1994, the use of PWS1 as a public supply well was terminated because of persistent high nitrate levels (Section 2.1.1).

The potential impact of the former use of private wells and well PWS1 on the groundwater flow system at Bendena has not been fully evaluated. Because the use of these wells spans much of the period following the termination of the grain storage operations at the former CCC/USDA facility, as well as the later grain storage activities on this property and at the Bendena Grain facilities (during which carbon tetrachloride contamination was detected in the groundwater and at PWS1), the possible hydraulic influence of these wells on the natural patterns of groundwater flow should be considered in evaluating the present distribution of this contaminant and the historic and modern patterns of groundwater flow and possible contaminant migration. 


\subsubsection{Summary}

The presently available site-specific data for the investigation area are consistent with the regional geologic and hydrogeologic setting, suggesting that the local groundwater flow system is developed within a relatively thick sequence of unconsolidated clays, silts, and silty clay till that contain heterogeneously distributed and relatively discontinuous deposits of more permeable silts, sands, and gravels. Previous studies have nominally identified several possible waterbearing units (perched, shallow, and lower aquifers) in this sequence; however, the detailed distribution, internal characteristics, and potential groundwater flow direction(s) in these units and the greater flow system remain unclear. Measurements of the local groundwater levels in a limited number of permanent monitoring wells indicate that significant but variable vertical gradients in the hydrogeologic system, coupled with the lithologic heterogeneity noted above, are likely to influence the detailed pathways for groundwater flow and potential contaminant migration in the saturated sediments. 
TABLE 2.1 Analytical results for VOCs in groundwater, 1985-2010.

\begin{tabular}{|c|c|c|c|c|}
\hline \multirow[b]{2}{*}{$\begin{array}{l}\text { Reported Sampling } \\
\text { Location }\end{array}$} & \multirow[b]{2}{*}{$\begin{array}{l}\text { Sample } \\
\text { Date }\end{array}$} & \multicolumn{2}{|c|}{ Concentration $(\mu \mathrm{g} / \mathrm{L})$} & \multirow[b]{2}{*}{ Notes on Result/Source } \\
\hline & & $\begin{array}{c}\text { Carbon } \\
\text { Tetrachloride }\end{array}$ & Chloroform & \\
\hline \multicolumn{5}{|c|}{ Results for well PWS1 and RWD 2 Distribution System, 1985-1998 } \\
\hline RWD 2 & $2 / 26 / 1985$ & 10.9 & $N R^{a}$ & Plant tap \\
\hline PWS1 & $3 / 11 / 1985$ & 11.5 & NR & \\
\hline PWS1 & 3/11/1985 & 10.9 & NR & \\
\hline RWD 2 distribution & 3/22/1985 & 14 & NR & Consumer Oil Co. \\
\hline PWS1 & $3 / 22 / 1985$ & 11.5 & NR & Godfrey residence \\
\hline RWD 2 distribution & 7/29/1985 & 7.8 & NR & \\
\hline PWS1 & 7/29/1985 & 6.4 & NR & In KDWW'b \\
\hline RWD 2 distribution & 9/11/1985 & 11.1 & NR & \\
\hline PWS1 & 4/15/1986 & 11 & NR & \\
\hline PWS1 & $7 / 23 / 1986$ & 12 & NR & \\
\hline PWS1 & $12 / 17 / 1986$ & 7.7 & NR & \\
\hline PWS1 & 12/17/1986 & 6.4 & NR & \\
\hline PWS1 & $12 / 17 / 1986$ & 14.4 & NR & In KDWW \\
\hline PWS1 & 4/22/1987 & 17.4 & NR & In KDWW \\
\hline PWS1 & 1/12/1988 & 14.8 & NR & \\
\hline RWD 2 distribution & 2/9/1988 & 12.7 & NR & St. John's Church \\
\hline PWS1 & 2/9/1988 & 13.3 & NR & In KDWW \\
\hline PWS1 & $5 / 18 / 1988$ & 20.3 & NR & \\
\hline PWS1 & $5 / 4 / 1989$ & 23.8 & NR & In KDWW \\
\hline PWS1 & 9/17/1991 & 17.2 & NR & In KDWW \\
\hline RWD 2-RWD 5 & 9/17/1991 & $<0.5$ & NR & Blended \\
\hline RWD 2-RWD 5 & 9/17/1991 & $<0.5$ & NR & Blended \\
\hline RWD 2-RWD 5 & 9/25/1991 & 0.8 & NR & Blended \\
\hline RWD 2-RWD 5 & 11/5/1991 & $<0.5$ & NR & Blended \\
\hline PWS1 & 4/22/1992 & 9.1 & NR & \\
\hline RWD 2-RWD 5 & 4/22/1992 & $<0.5$ & NR & Blended \\
\hline RWD 2 distribution & 6/18/1992 & $<0.5$ & NR & \\
\hline RWD 2 distribution & 6/22/1992 & $<0.5$ & NR & \\
\hline RWD 2 distribution & $6 / 22 / 1992$ & $<0.5$ & NR & St. John's Church \\
\hline RWD 2 distribution & 9/11/1992 & $<0.5$ & NR & \\
\hline RWD 2 distribution & $4 / 27 / 1994$ & $<0.5$ & NR & Weiland Grocery \\
\hline PWS1 & 9/4/1996 & $<0.7$ & NR & \\
\hline PWS1 & 4/15/1998 & $<5$ & 56 & \\
\hline PWS1-RWD 2 & $6 / 11 / 1998$ & $N^{c}$ & NR & Sampling point unspecified \\
\hline PWS1-RWD 2 & 6/15/1998 & 20.5 & NR & Sampling point unspecified \\
\hline \multicolumn{5}{|c|}{ Results from the KDHE site inspection (1988) } \\
\hline Albers, R., well & $1 / 12 / 1988$ & 2.9 & NR & \\
\hline Albers, R., well & $5 / 18 / 1988$ & 2.6 & NR & \\
\hline Atwater, J., well & 1/12/1988 & $<0.7$ & NR & \\
\hline Clark, J., well & $4 / 14 / 1988$ & $<0.7$ & NR & \\
\hline Dickson, B., well & $4 / 8 / 1988$ & $<0.7$ & NR & \\
\hline Johnson, C., well & $1 / 12 / 1988$ & $<0.7$ & NR & \\
\hline Johnson, G. feedlot well & $5 / 18 / 1988$ & $<0.7$ & NR & \\
\hline Johnson, R., well & 1/12/1988 & $<0.7$ & NR & \\
\hline Kentzler, M., well & 4/8/1988 & $<0.7$ & NR & \\
\hline Myers, P., well & $1 / 12 / 1988$ & $<0.7$ & NR & \\
\hline Pope, D., well & $1 / 12 / 1988$ & $<0.7$ & NR & \\
\hline Foster, L., well & $1 / 12 / 1988$ & $<0.7$ & NR & \\
\hline
\end{tabular}


TABLE 2.1 (Cont.)

\begin{tabular}{|c|c|c|c|}
\hline \multirow[b]{2}{*}{$\begin{array}{l}\text { Reported Sampling } \\
\text { Location }\end{array}$} & \multirow[b]{2}{*}{$\begin{array}{l}\text { Sample } \\
\text { Date }\end{array}$} & \multicolumn{2}{|c|}{ Concentration $(\mu \mathrm{g} / \mathrm{L}$} \\
\hline & & $\begin{array}{l}\text { Carbon } \\
\text { Tetrachloride }\end{array}$ & Chlo \\
\hline \multicolumn{4}{|c|}{ Results from the KDHE site inspection (1988) (cont.) } \\
\hline Holzhey, G., well & 4/8/1988 & $<0.7$ & NR \\
\hline Rush, D., well (bottom) & $1 / 12 / 1988$ & $<0.7$ & NR \\
\hline Rush, D., well (top) & $1 / 12 / 1988$ & $<0.7$ & NR \\
\hline Rush, F., well & $1 / 12 / 1988$ & $<0.7$ & NR \\
\hline Staudenmaier, C., well & $1 / 12 / 1988$ & $<0.7$ & NR \\
\hline Staudenmaier, P., well & $1 / 12 / 1988$ & $<0.7$ & NR \\
\hline MW1 & 4/14/1988 & $<0.7$ & NR \\
\hline MW1 & $5 / 18 / 1988$ & $<0.7$ & NR \\
\hline MW2 & $5 / 18 / 1988$ & 62.9 & NR \\
\hline MW3 & 4/8/1988 & $<0.7$ & NR \\
\hline MW3 & $5 / 18 / 1988$ & $<0.7$ & NR \\
\hline
\end{tabular}

Results from the KDHE comprehensive investigation (1997-1998)

\begin{tabular}{|c|c|c|c|}
\hline Dickson, B., well & 11/18/1997 & $<1$ & $<1$ \\
\hline Dickson, B., well & 4/13/1998 & $<1$ & 0.99 \\
\hline Holzhey, G., well & 11/18/1997 & $<1$ & $<1$ \\
\hline Holzhey, G., well & 4/13/1998 & $<1$ & $<1$ \\
\hline Johnson well & 4/13/1998 & $<1$ & 57 \\
\hline Phillips well & 4/13/1998 & $<1$ & $<1$ \\
\hline Phillips well & $11 / 18 / 1998$ & $<1$ & $<1$ \\
\hline MW1 & $11 / 18 / 1997$ & $<\overline{1}$ & $<1$ \\
\hline MW1 & 4/13/1998 & $<1$ & $<1$ \\
\hline MW2 & $11 / 18 / 1997$ & 120 & 1.9 \\
\hline MW2 & 3/3/1998 & 120 & NR \\
\hline MW2 & 4/15/1998 & 75 & 2 \\
\hline MW3 & $11 / 18 / 1997$ & $<1$ & $<\overline{1}$ \\
\hline MW3 & 4/13/1998 & $<1$ & $<1$ \\
\hline MW4D & 3/10/1998 & $<1$ & $<1$ \\
\hline MW4D & $4 / 15 / 1998$ & $<1$ & $<1$ \\
\hline MW4P & 3/3/1998 & 18 & 6.4 \\
\hline MW4P & $4 / 15 / 1998$ & 2.6 & $<1$ \\
\hline MW4S & 3/12/1998 & 410 & 10 \\
\hline MW4S & $4 / 15 / 1998$ & 220 & $<1$ \\
\hline MW4S (duplicate) & $4 / 15 / 1998$ & 210 & $<1$ \\
\hline MW5 & 3/3/1998 & $<1$ & $<1$ \\
\hline MW5 & $4 / 15 / 1998$ & $<1$ & $<1$ \\
\hline MW6 & 2/26/1998 & $<1$ & 1.6 \\
\hline MW6 & 4/14/1998 & $<1$ & $<1$ \\
\hline MW7 & 2/26/1998 & $<1$ & $<1$ \\
\hline MW7 & $4 / 14 / 1998$ & $<1$ & $<1$ \\
\hline MW8 & $2 / 25 / 1998$ & $<1$ & $<1$ \\
\hline MW8 & 4/14/1998 & $<1$ & $<1$ \\
\hline MW9D & 3/11/1998 & $<1$ & 1 \\
\hline MW9D & $4 / 15 / 1998$ & $<1$ & $<1$ \\
\hline MW9S & $3 / 12 / 1998$ & 2.5 & 2 \\
\hline MW9S & $4 / 15 / 1998$ & 2.2 & 0.9 \\
\hline
\end{tabular}


TABLE 2.1 (Cont.)

\begin{tabular}{|c|c|c|c|c|}
\hline \multirow[b]{2}{*}{$\begin{array}{l}\text { Reported Sampling } \\
\text { Location }\end{array}$} & \multirow[b]{2}{*}{$\begin{array}{l}\text { Sample } \\
\text { Date }\end{array}$} & \multicolumn{2}{|c|}{ Concentration $(\mu \mathrm{g} / \mathrm{L})$} & \multirow[b]{2}{*}{ Notes on Result/Source } \\
\hline & & $\begin{array}{c}\text { Carbon } \\
\text { Tetrachloride }\end{array}$ & Chloroform & \\
\hline \multicolumn{5}{|c|}{ Results of sampling in selected monitoring wells (1999-2005) } \\
\hline MW2 & 1/15/1999 & 123 & NR & \\
\hline MW4P & 1/15/1999 & 35.7 & NR & \\
\hline MW4S & 1/15/1999 & 231 & NR & \\
\hline MW4D & $1 / 15 / 1999$ & ND & NR & \\
\hline MW2 & 4/19/2001 & 75.8 & NR & \\
\hline MW4P & 4/19/2001 & 49.9 & NR & \\
\hline MW4S & 4/19/2001 & 198 & NR & \\
\hline MW9S & 4/19/2001 & 8.6 & NR & \\
\hline MW9D & $4 / 19 / 2001$ & ND & NR & \\
\hline MW2 & $4 / 24 / 2002$ & 90.7 & NR & \\
\hline MW4P & $4 / 24 / 2002$ & 86.3 & NR & \\
\hline MW5 & $4 / 24 / 2002$ & ND & NR & \\
\hline MW9S & $4 / 24 / 2002$ & 9.1 & NR & \\
\hline MW9D & $4 / 24 / 2002$ & ND & NR & \\
\hline MW2 & 6/13/2003 & 82 & NR & \\
\hline MW4P & 6/12/2003 & 73 & NR & \\
\hline MW4S & 6/12/2003 & 180 & NR & \\
\hline MW4D & 6/13/2003 & 83 & NR & \\
\hline MW5 & 6/12/2003 & ND & NR & \\
\hline MW9S & 6/12/2003 & 11 & NR & \\
\hline MW9D & $6 / 12 / 2003$ & ND & NR & \\
\hline MW2 & $5 / 17 / 2005$ & 160 & NR & \\
\hline MW4P & $5 / 17 / 2005$ & ND & NR & \\
\hline MW4S & $5 / 17 / 2005$ & 160 & NR & \\
\hline MW5 & $5 / 17 / 2005$ & ND & NR & \\
\hline MW9S & $5 / 17 / 2005$ & 12 & NR & \\
\hline MW9D & $5 / 17 / 2005$ & ND & NR & \\
\hline MW4P & $6 / 10 / 2009$ & 160 & NR & \\
\hline MW4S & 6/10/2009 & 0.85 & NR & \\
\hline MW4D & 6/10/2009 & ND & NR & \\
\hline MW4D & 6/10/09 dup & ND & NR & \\
\hline \multicolumn{5}{|c|}{ Results from the KDHE supplemental site investigation (2009-2010) ${ }^{\mathrm{d}}$} \\
\hline GW01S & $12 / 22 / 09$ & $100 / 67$ & $5.2 / 3.4$ & 74-79 ft BGL \\
\hline GW02S & $12 / 22 / 09$ & 240/190 & $6.2 / 4.5$ & $69.5-74.5 \mathrm{ft} \mathrm{BGL}$ \\
\hline GW03S & $12 / 22 / 09$ & $3.5 / 2.5$ & ND/0.3 je & $54-58 \mathrm{ft} \mathrm{BGL}$ \\
\hline GW04S & Dry & Dry & Dry & $54-58 \mathrm{ft} \mathrm{BGL}$ \\
\hline GW05P & $12 / 17 / 09$ & ND/ND & ND/ND & $11-15 \mathrm{ft} \mathrm{BGL}$ \\
\hline GW05S & $12 / 17 / 09$ & $91 / 110$ & $3 / 2.7$ & $53-57 \mathrm{ft} \mathrm{BGL}$ \\
\hline GW06S & $1 / 5 / 10$ & $39 / 50$ & $1.9 / 1.8$ & $65-69 \mathrm{ft} \mathrm{BGL}$ \\
\hline GW07S & $1 / 5 / 10$ & $190 / 244$ & $2.5 / 2.6$ & $63-67 \mathrm{ft} \mathrm{BGL}$ \\
\hline GW08S & 12/18/09 & 20/16 & $1.4 / 1.0$ & 57-61 ft BGL \\
\hline GW09S & 12/21/09 & ND/ND & ND/ND & $56-61 \mathrm{ft} \mathrm{BGL}$ \\
\hline GW10S & 12/21/09 & ND/ND & ND/0.3 J & $60-65 \mathrm{ft} \mathrm{BGL}$ \\
\hline
\end{tabular}




\section{TABLE 2.1 (Cont.)}

a NR, not reported.

b Data available in the KDHE Kansas Drinking Water Watch online database.

c ND, not detected.

d Results of analyses by KDHE/AGEM Laboratory.

e $\mathrm{J}$, estimated concentration below the AGEM Laboratory quantitation limit of $1.0 \mu \mathrm{g} / \mathrm{L}$ for this compound. 
TABLE 2.2 Analytical results for soil gas samples collected by the KDHE on November 5, 1987. ${ }^{\mathrm{a}}$

\begin{tabular}{|c|c|c|c|c|c|c|}
\hline \multirow[b]{2}{*}{ Location } & \multirow{2}{*}{$\begin{array}{l}\text { Sample } \\
\text { Depth } \\
\text { (ft BGL) }\end{array}$} & \multicolumn{5}{|c|}{ Concentration (ppbv) } \\
\hline & & $\mathrm{CCl}_{4}$ & TCA & TCE & Toluene & $\mathrm{THC}$ \\
\hline SG1 & 4 & 0.0008 & 0.002 & 0.02 & 4 & 4 \\
\hline SG2 & 6 & 0.00008 & 0.0008 & 0.002 & $<0.05$ & $<0.05$ \\
\hline SG3 & 5 & 0.01 & 0.001 & 0.008 & $<0.05$ & $<0.05$ \\
\hline SG4 & 4 & 0.2 & 0.001 & 0.02 & $<0.05$ & $<0.05$ \\
\hline SG5 & 3 & 0.0002 & 0.002 & 0.004 & $<0.05$ & $<0.05$ \\
\hline SG6 & 6 & 0.0002 & 0.03 & 0.05 & $<0.05$ & $<0.05$ \\
\hline SG7 & 4 & 0.0002 & 0.002 & 0.002 & $<0.05$ & $<0.05$ \\
\hline SG8 & 5 & 0.003 & 0.001 & 0.002 & $<0.05$ & $<0.05$ \\
\hline SG9 & 6 & 0.0005 & 0.004 & 0.005 & 0.3 & 0.8 \\
\hline SG10 & 5 & 0.0003 & 0.002 & 0.0008 & 0.2 & 0.8 \\
\hline SG11 & 6 & $<0.00002$ & 0.002 & 0.003 & $<0.05$ & $<0.05$ \\
\hline SG12 & 4 & 0.0004 & 0.002 & 0.003 & 0.2 & 2 \\
\hline
\end{tabular}

a Abbreviations: ppbv, parts per billion by volume $B G L$, below ground level $\mathrm{CCl}_{4}$, carbon tetrachloride TCA, 1,1,1-trichloroethane TCE, trichloroethylene $\mathrm{THC}$, total hydrocarbons 
TABLE 2.3 Construction data for permanent monitoring wells.

\begin{tabular}{|c|c|c|c|c|c|c|}
\hline \multirow[b]{2}{*}{ Well } & \multirow[b]{2}{*}{$\begin{array}{c}\text { Year } \\
\text { Installed }\end{array}$} & \multirow{2}{*}{$\begin{array}{l}\text { Screen } \\
\text { Length } \\
\text { (ft) }\end{array}$} & \multicolumn{3}{|c|}{ Depth (ft BGL) } & \multirow{2}{*}{$\begin{array}{l}\text { Reference } \\
\text { Elevation } \\
\text { (ft AMSL) }\end{array}$} \\
\hline & & & Total $^{\mathrm{a}}$ & $\begin{array}{l}\text { Screen } \\
\text { Interval }^{\mathrm{a}}\end{array}$ & $\begin{array}{c}\text { Filter Pack } \\
\text { Intervala }^{\mathrm{a}}\end{array}$ & \\
\hline $\mathrm{MW}^{\mathrm{C}}$ & 1988 & 15 & 99 & 84-99 & $26-99$ & 1130.18 \\
\hline $\mathrm{MW}^{\mathrm{C}}$ & 1988 & 15 & 99.55 & $84.55-99.55$ & $25-99.55$ & 1110.69 \\
\hline $\mathrm{MW}^{\mathrm{C}}$ & 1988 & 10 & 71.4 & 61.4-71.4 & $25-71.4$ & 1086.85 \\
\hline MW4P & 1998 & 10 & 30 & $20-30$ & 18.3 & 1123.17 \\
\hline MW4S & 1998 & 20 & 82 & $60-80$ & $58-82$ & 1123.23 \\
\hline MW4D & 1998 & 15 & 140 & $111-126$ & $108.5-129$ & 1123.23 \\
\hline MW5 & 1998 & 15 & 140 & $115-130$ & $110-133$ & 1124.56 \\
\hline MW6 & 1998 & 15 & 135 & $115-130$ & $111-135$ & 1108.85 \\
\hline MW7 & 1998 & 15 & 140 & 123.138 & $120-140$ & 1116.31 \\
\hline MW8 & 1998 & 15 & 122 & 106.121 & $100-122$ & 1089.59 \\
\hline MW9S & 1998 & 20 & 65 & $45-65$ & $42-65$ & 1107.64 \\
\hline MW9D & 1998 & 15 & 138 & $105-120$ & $102-123$ & 1107.34 \\
\hline MW10P & 2012 & 10 & 25.4 & $15.1-25.1$ & $13-25.4$ & $N R^{d}$ \\
\hline MW10S & 2012 & 20 & 65.4 & $44.85-64.85$ & $48-65.4$ & NR \\
\hline
\end{tabular}

a Data are as reported in KDHE WWC-5 registration forms.

b Elevations for wells MW1-MW9 are as reported in comprehensive investigation (KDHE 1998).

c Wells MW1-MW3 were plugged and abandoned by the KDHE in 2006.

d NR, data not recorded. 
TABLE 2.4 Analytical results for VOCs in vertical-profile soil samples collected at location MW4D in March 1998.

\begin{tabular}{|c|c|c|c|c|c|}
\hline \multirow{3}{*}{$\begin{array}{c}\text { Sample } \\
\text { Depth } \\
\text { (ft BGL) }\end{array}$} & \multirow{3}{*}{$\begin{array}{c}\text { Sample } \\
\text { Date }\end{array}$} & \multicolumn{4}{|c|}{ Concentration $^{\mathrm{a}}(\mu \mathrm{g} / \mathrm{kg})$} \\
\hline & & Carbon & & Methylene & \\
\hline & & Tetrachloride & Chloroform & Chloride $^{b}$ & Tetrachloroethene \\
\hline $1-3$ & $3 / 2 / 98$ & $<6.4$ & $<6.4$ & $<6.4$ & $<6.4$ \\
\hline $5-7$ & $3 / 2 / 98$ & $<6.6$ & $<6.6$ & $<6.6$ & $<6.6$ \\
\hline $10-12$ & $3 / 2 / 98$ & $<6.6$ & $<6.6$ & $2 \mathrm{~J}$ & $<6.6$ \\
\hline $15-17$ & $3 / 2 / 98$ & $<6.6$ & $<6.6$ & 2.1 & $<6.6$ \\
\hline $20-22$ & $3 / 2 / 98$ & $<6.3$ & $<6.3$ & $<6.3$ & $<6.3$ \\
\hline $25-27$ & $3 / 2 / 98$ & 3.5 & $<6.2$ & $<6.2$ & $<6.2$ \\
\hline $30-32$ & $3 / 2 / 98$ & $<6.1$ & $<6.1$ & $<6.1$ & $<6.1$ \\
\hline $35-37$ & $3 / 2 / 98$ & $<6.3$ & $<6.3$ & $<6.3$ & $<6.3$ \\
\hline $40-42$ & $3 / 2 / 98$ & $<6.3$ & $<6.3$ & $<6.3$ & $<6.3$ \\
\hline $45-47$ & $3 / 2 / 98$ & $<6.3$ & $<6.3$ & $<6.3$ & $<6.3$ \\
\hline $50-52$ & $3 / 4 / 98$ & 100 & $<6.2$ & 10 & $<6.2$ \\
\hline $55-57$ & $3 / 4 / 98$ & $<6.3$ & $<6.3$ & 12 & $<6.3$ \\
\hline $60-62$ & $3 / 4 / 98$ & 7 & $<6.3$ & 11 & 1.9 \\
\hline $65-67$ & $3 / 4 / 98$ & 3.2 & $<6.1$ & 11 & $<6.1$ \\
\hline
\end{tabular}

a Data are as reported by the KDHE (1998).

b Methylene chloride was detected in the method blank associated with analysis of the samples. 
Version 01, 08/28/13

TABLE 2.5 Analytical results for VOCs in groundwater samples collected by the KDHE from private wells in February 2013. ${ }^{a}$

\begin{tabular}{|c|c|c|c|c|c|}
\hline \multirow{2}{*}{$\begin{array}{l}\text { Reported } \\
\text { Sampling } \\
\text { Location }\end{array}$} & \multirow{2}{*}{$\begin{array}{c}\text { Sample } \\
\text { Date }\end{array}$} & \multicolumn{2}{|c|}{ Concentration $(\mu \mathrm{g} / \mathrm{L})$} & \multirow{2}{*}{$\begin{array}{l}\text { Methylene } \\
\text { Chloride }\end{array}$} & \multirow[b]{2}{*}{ Notes on Result/Source } \\
\hline & & Tetrachloride & Chloroform & & \\
\hline Albers & $2 / 13 / 13$ & 4.4 & ND & ND & - \\
\hline B. Dickson & $2 / 14 / 13$ & ND & 1.1 & ND & One Dickson well in work plan. \\
\hline R. Dickson & $2 / 14 / 13$ & ND & ND & ND & One Dickson well in work plan. \\
\hline D. Rush & $2 / 13 / 13$ & ND & ND & ND & - \\
\hline Johnson & $2 / 13 / 13$ & ND & ND & ND & Two Johnson wells in work plan. \\
\hline L. Rush & $2 / 13 / 13$ & ND & ND & ND & Name not in work plan. \\
\hline Staudenmaier & $2 / 13 / 13$ & ND & ND & ND & Two Staudenmaier wells in work plan. \\
\hline Thompson & $2 / 13 / 13$ & ND & ND & ND & Name not in work plan. \\
\hline
\end{tabular}

a Data are as provided to Argonne by the CCC/USDA (2013). 
TABLE 2.6 Summary of groundwater level measurements for the permanent monitoring wells and well PWS1.

\begin{tabular}{|c|c|c|c|c|c|c|}
\hline Well & $\begin{array}{c}\text { Reference }\left(\mathrm{TOC}^{\mathrm{a}}\right) \\
\text { Elevation } \\
\text { (ft AMSL) }\end{array}$ & $\begin{array}{l}\text { Reference } \\
\text { Elevation } \\
\text { Source }^{b}\end{array}$ & $\begin{array}{c}\text { Measurement } \\
\text { Date }\end{array}$ & $\begin{array}{l}\text { Measured } \\
\text { Depth to } \\
\text { Water } \\
\text { (ft TOC) })^{\mathrm{a}}\end{array}$ & $\begin{array}{l}\text { Calculated } \\
\text { Water Level } \\
\text { Elevation } \\
\text { (ft AMSL) }\end{array}$ & $\begin{array}{l}\text { Measured } \\
\text { Water Level } \\
\text { Source }^{b}\end{array}$ \\
\hline \multirow[t]{4}{*}{$\mathrm{MW}^{\mathrm{C}}$} & 1130.18 & $\mathrm{SI}, \mathrm{Cl}$ & 4/20/88 & 62.40 & 1067.78 & SI \\
\hline & & & 8/17/88 & 83.30 & 1046.88 & SI \\
\hline & & & 4/13/98 & 57.36 & 1072.82 & $\mathrm{Cl}$ \\
\hline & & & $4 / 13 / 98$ & 57.48 & 1072.70 & $\mathrm{Cl}$ \\
\hline \multirow[t]{8}{*}{$M W 2^{c}$} & 1110.69 & $\mathrm{SI}, \mathrm{Cl}$ & 4/20/88 & 39.90 & 1070.79 & SI \\
\hline & & & $5 / 18 / 88$ & 50.65 & 1060.04 & SSI \\
\hline & & & 8/17/88 & 51.00 & 1059.69 & $\mathrm{SI}$ \\
\hline & & & $11 / 18 / 97$ & 50.96 & 1059.73 & SSI \\
\hline & & & $4 / 13 / 98$ & 44.52 & 1066.17 & $\mathrm{Cl}$ \\
\hline & & & $4 / 15 / 98$ & 44.14 & 1066.55 & $\mathrm{Cl}$ \\
\hline & & & $4 / 24 / 02$ & 53.58 & 1057.11 & SSI \\
\hline & & & $5 / 17 / 05$ & 54.00 & 1056.69 & SSI \\
\hline \multirow[t]{7}{*}{$\mathrm{MW}^{\mathrm{C}}$} & 1085.37 & SI & 4/8/88 & 25.00 & 1060.37 & SSI \\
\hline & & & $4 / 20 / 88$ & 25.00 & 1060.37 & SI \\
\hline & & & 8/17/88 & 27.85 & 1057.52 & $\mathrm{SI}$ \\
\hline & 1086.85 & $\mathrm{Cl}$ & $3 / 20 / 98$ & 21.46 & 1065.39 & $\mathrm{Cl}$ \\
\hline & & & 3/31/98 & 20.84 & 1066.01 & $\mathrm{Cl}$ \\
\hline & & & $4 / 13 / 98$ & 18.32 & 1068.53 & $\mathrm{Cl}$ \\
\hline & & & 4/13/98 & 18.33 & 1068.52 & $\mathrm{Cl}$ \\
\hline \multirow[t]{6}{*}{ MW4D } & 1123.23 & $\mathrm{Cl}$ & $3 / 20 / 98$ & 65.32 & 1057.91 & $\mathrm{Cl}$ \\
\hline & & & $3 / 31 / 98$ & 63.36 & 1059.87 & $\mathrm{Cl}$ \\
\hline & & & $4 / 13 / 98$ & 61.68 & 1061.55 & $\mathrm{Cl}$ \\
\hline & & & $4 / 15 / 98$ & 61.57 & 1061.66 & $\mathrm{Cl}$ \\
\hline & & & $6 / 10 / 09$ & 59.20 & 1064.03 & SSI \\
\hline & & & $7 / 17 / 12$ & 69.80 & 1053.43 & JF2 \\
\hline \multirow[t]{10}{*}{ MW4S } & 1123.23 & $\mathrm{Cl}$ & 3/20/98 & 46.90 & 1076.33 & $\mathrm{Cl}$ \\
\hline & & & $3 / 31 / 98$ & 44.32 & 1078.91 & $\mathrm{Cl}$ \\
\hline & & & $4 / 13 / 98$ & 42.42 & 1080.81 & $\mathrm{Cl}$ \\
\hline & & & $4 / 15 / 98$ & 41.75 & 1081.48 & $\mathrm{Cl}$ \\
\hline & & & $5 / 17 / 05$ & 58.36 & 1064.87 & SSI \\
\hline & & & $5 / 23 / 06$ & 59.51 & 1063.72 & SSI \\
\hline & & & $5 / 30 / 07$ & 43.30 & 1079.93 & SSI \\
\hline & & & $7 / 8 / 08$ & 40.22 & 1083.01 & SSI \\
\hline & & & $6 / 10 / 09$ & 38.48 & 1084.75 & SSI \\
\hline & & & $7 / 17 / 12$ & 45.47 & 1077.76 & $\mathrm{JF} 2$ \\
\hline \multirow[t]{11}{*}{ MW4P } & 1123.17 & $\mathrm{Cl}$ & $3 / 20 / 98$ & 13.63 & 1109.54 & $\mathrm{Cl}$ \\
\hline & & & $3 / 31 / 98$ & 9.92 & 1113.25 & $\mathrm{Cl}$ \\
\hline & & & $4 / 13 / 98$ & 10.65 & 1112.52 & $\mathrm{Cl}$ \\
\hline & & & $4 / 15 / 98$ & 10.81 & 1112.36 & $\mathrm{Cl}$ \\
\hline & & & $4 / 24 / 02$ & 19.70 & 1103.47 & SSI \\
\hline & & & $5 / 17 / 05$ & 14.10 & 1109.07 & SSI \\
\hline & & & $5 / 23 / 06$ & 15.76 & 1107.41 & SSI \\
\hline & & & $5 / 30 / 07$ & 11.45 & 1111.72 & SSI \\
\hline & & & $7 / 8 / 08$ & 14.92 & 1108.25 & SSI \\
\hline & & & $6 / 10 / 09$ & 12.36 & 1110.81 & SSI \\
\hline & & & $7 / 17 / 12$ & 19.30 & 1103.87 & $\mathrm{JF} 2$ \\
\hline
\end{tabular}


TABLE 2.6 (Cont.)

\begin{tabular}{|c|c|c|c|c|c|c|}
\hline Well & $\begin{array}{c}\text { Reference }\left(\mathrm{TOC}^{\mathrm{a}}\right) \\
\text { Elevation } \\
\text { (ft AMSL) }\end{array}$ & $\begin{array}{l}\text { Reference } \\
\text { Elevation } \\
\text { Source }^{b}\end{array}$ & $\begin{array}{c}\text { Measurement } \\
\text { Date }\end{array}$ & $\begin{array}{l}\text { Measured } \\
\text { Depth to } \\
\text { Water } \\
\text { (ft TOC) }^{\mathrm{a}}\end{array}$ & $\begin{array}{l}\text { Calculated } \\
\text { Water Level } \\
\text { Elevation } \\
\text { (ft AMSL) }\end{array}$ & $\begin{array}{c}\text { Measured } \\
\text { Water Level } \\
\text { Source }^{b}\end{array}$ \\
\hline \multirow[t]{11}{*}{ MW5 } & 1124.56 & $\mathrm{Cl}$ & $3 / 20 / 98$ & 66.56 & 1058.00 & $\mathrm{Cl}$ \\
\hline & & & $3 / 31 / 98$ & 64.80 & 1059.76 & $\mathrm{Cl}$ \\
\hline & & & 4/13/98 & 63.15 & 1061.41 & $\mathrm{Cl}$ \\
\hline & & & 4/13-15/98 & 62.99 & 1061.57 & $\mathrm{Cl}$ \\
\hline & & & $4 / 24 / 02$ & 71.29 & 1053.27 & SSI \\
\hline & & & $5 / 17 / 05$ & 68.41 & 1056.15 & SSI \\
\hline & & & $5 / 23 / 06$ & 69.63 & 1054.93 & SSI \\
\hline & & & $5 / 24 / 07$ & 60.57 & 1063.99 & SSI \\
\hline & & & $7 / 8 / 08$ & 68.20 & 1056.36 & SSI \\
\hline & & & $6 / 10 / 09$ & 60.72 & 1063.84 & SSI \\
\hline & & & $7 / 17 / 12$ & 66.15 & 1058.41 & JF2 \\
\hline \multirow[t]{5}{*}{ MW6 } & 1108.85 & $\mathrm{Cl}$ & 3/20/98 & 51.09 & 1057.76 & $\mathrm{Cl}$ \\
\hline & & & $3 / 31 / 98$ & 49.32 & 1059.53 & $\mathrm{Cl}$ \\
\hline & & & $4 / 13 / 98$ & 47.77 & 1061.08 & $\mathrm{Cl}$ \\
\hline & & & $4 / 13 / 98$ & 47.14 & 1061.71 & $\mathrm{Cl}$ \\
\hline & & & $8 / 18 / 09$ & 47.66 & 1061.19 & SSI \\
\hline \multirow[t]{5}{*}{ MW7 } & 1116.31 & $\mathrm{Cl}$ & 3/20/98 & 59.15 & 1057.16 & $\mathrm{Cl}$ \\
\hline & & & $3 / 31 / 98$ & 57.33 & 1058.98 & $\mathrm{Cl}$ \\
\hline & & & $4 / 13 / 98$ & 55.59 & 1060.72 & $\mathrm{Cl}$ \\
\hline & & & $4 / 13 / 98$ & 55.51 & 1060.80 & $\mathrm{Cl}$ \\
\hline & & & 8/18/09 & 55.40 & 1060.91 & SSI \\
\hline \multirow[t]{5}{*}{ MW8 } & 1089.59 & $\mathrm{Cl}$ & $3 / 20 / 98$ & 32.24 & 1057.35 & $\mathrm{Cl}$ \\
\hline & & & $3 / 31 / 98$ & 30.35 & 1059.24 & $\mathrm{Cl}$ \\
\hline & & & $4 / 13 / 98$ & 28.98 & 1060.61 & $\mathrm{Cl}$ \\
\hline & & & $4 / 13 / 98$ & 28.46 & 1061.13 & $\mathrm{Cl}$ \\
\hline & & & $8 / 18 / 09$ & 28.66 & 1060.93 & SSI \\
\hline \multirow[t]{10}{*}{ MW9D } & 1107.34 & $\mathrm{Cl}$ & $3 / 20 / 98$ & 49.68 & 1057.66 & $\mathrm{Cl}$ \\
\hline & & & $3 / 31 / 98$ & 47.82 & 1059.52 & $\mathrm{Cl}$ \\
\hline & & & $4 / 13 / 98$ & 46.14 & 1061.20 & $\mathrm{Cl}$ \\
\hline & & & $4 / 15 / 98$ & 45.97 & 1061.37 & $\mathrm{Cl}$ \\
\hline & & & $4 / 24 / 02$ & 53.37 & 1053.97 & SSI \\
\hline & & & $5 / 17 / 05$ & 52.85 & 1054.49 & SSI \\
\hline & & & $5 / 23 / 06$ & 66.60 & 1040.74 & SSI \\
\hline & & & $5 / 30 / 07$ & 43.95 & 1063.39 & SSI \\
\hline & & & 7/8/08 & 43.19 & 1064.15 & SSI \\
\hline & & & 6/10/09 & 42.88 & 1064.46 & SSI \\
\hline \multirow[t]{10}{*}{ MW9S } & 1107.64 & $\mathrm{Cl}$ & $3 / 20 / 98$ & 32.89 & 1074.75 & $\mathrm{Cl}$ \\
\hline & & & 3/31/98 & 29.61 & 1078.03 & $\mathrm{Cl}$ \\
\hline & & & $4 / 13 / 98$ & 27.97 & 1079.67 & $\mathrm{Cl}$ \\
\hline & & & $4 / 15 / 98$ & 27.02 & 1080.62 & $\mathrm{Cl}$ \\
\hline & & & $4 / 24 / 02$ & 39.70 & 1067.94 & SSI \\
\hline & & & $5 / 17 / 05$ & 41.00 & 1066.64 & SSI \\
\hline & & & $5 / 23 / 06$ & 31.55 & 1076.09 & SSI \\
\hline & & & $5 / 30 / 07$ & 27.65 & 1079.99 & SSI \\
\hline & & & $7 / 8 / 08$ & 27.20 & 1080.44 & SSI \\
\hline & & & $6 / 10 / 09$ & 24.91 & 1082.73 & SSI \\
\hline
\end{tabular}


TABLE 2.6 (Cont.)

\begin{tabular}{|c|c|c|c|c|c|c|}
\hline Well & $\begin{array}{c}\text { Reference }\left(\text { TOC }^{\mathrm{a}}\right) \\
\text { Elevation } \\
\text { (ft AMSL) }\end{array}$ & $\begin{array}{c}\text { Reference } \\
\text { Elevation } \\
\text { Source }^{\mathrm{b}}\end{array}$ & $\begin{array}{c}\text { Measurement } \\
\text { Date }\end{array}$ & $\begin{array}{c}\text { Measured } \\
\text { Depth to } \\
\text { Water } \\
\text { (ft TOC) })^{\mathrm{a}}\end{array}$ & $\begin{array}{l}\text { Calculated } \\
\text { Water Level } \\
\text { Elevation } \\
\text { (ft AMSL) }\end{array}$ & $\begin{array}{c}\text { Measured } \\
\text { Water Level } \\
\text { Source }^{b}\end{array}$ \\
\hline MW10S & Not determined & - & $7 / 17 / 12$ & 52.75 & - & $\mathrm{JF} 2$ \\
\hline MW10P & Not determined & - & $7 / 17 / 12$ & Dry & - & JF2 \\
\hline PWS1 & 1111.41 & $\mathrm{Cl}$ & $\begin{array}{l}4 / 20 / 88 \\
8 / 17 / 88\end{array}$ & $\begin{array}{l}47.00 \\
48.35\end{array}$ & $\begin{array}{l}1064.41 \\
1063.06\end{array}$ & $\begin{array}{l}\mathrm{SI} \\
\mathrm{SI}\end{array}$ \\
\hline
\end{tabular}

a TOC, top of casing.

b Sources:

SI, KDHE site inspection (KDHE 1988)

$\mathrm{Cl}$, KDHE comprehensive investigation (KDHE 1998)

SSI, KDHE supplemental site investigation (KDHE 2010)

JF2, Johnson Farms Phase II comprehensive investigation (Larsen 2012b)

c Wells MW-1, MW-2, and MW-3 were abandoned by the KDHE in early 2006. 


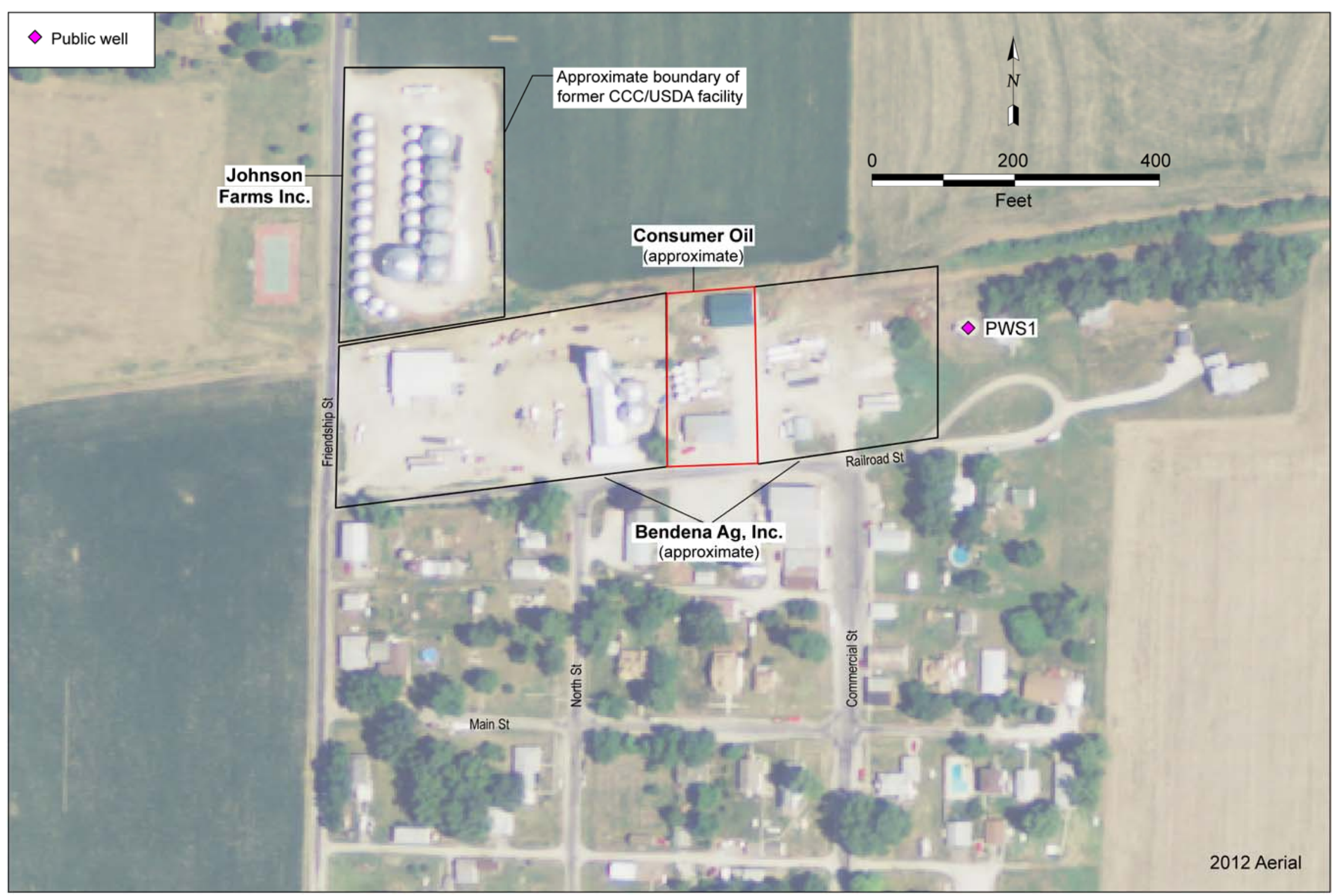

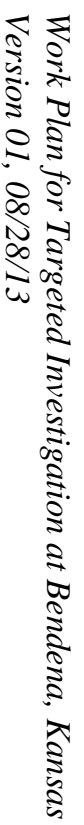

FIGURE 2.1 Locations and current ownership of properties of interest in the investigation, with well PWS1. Source of photograph: NAIP (2012). 


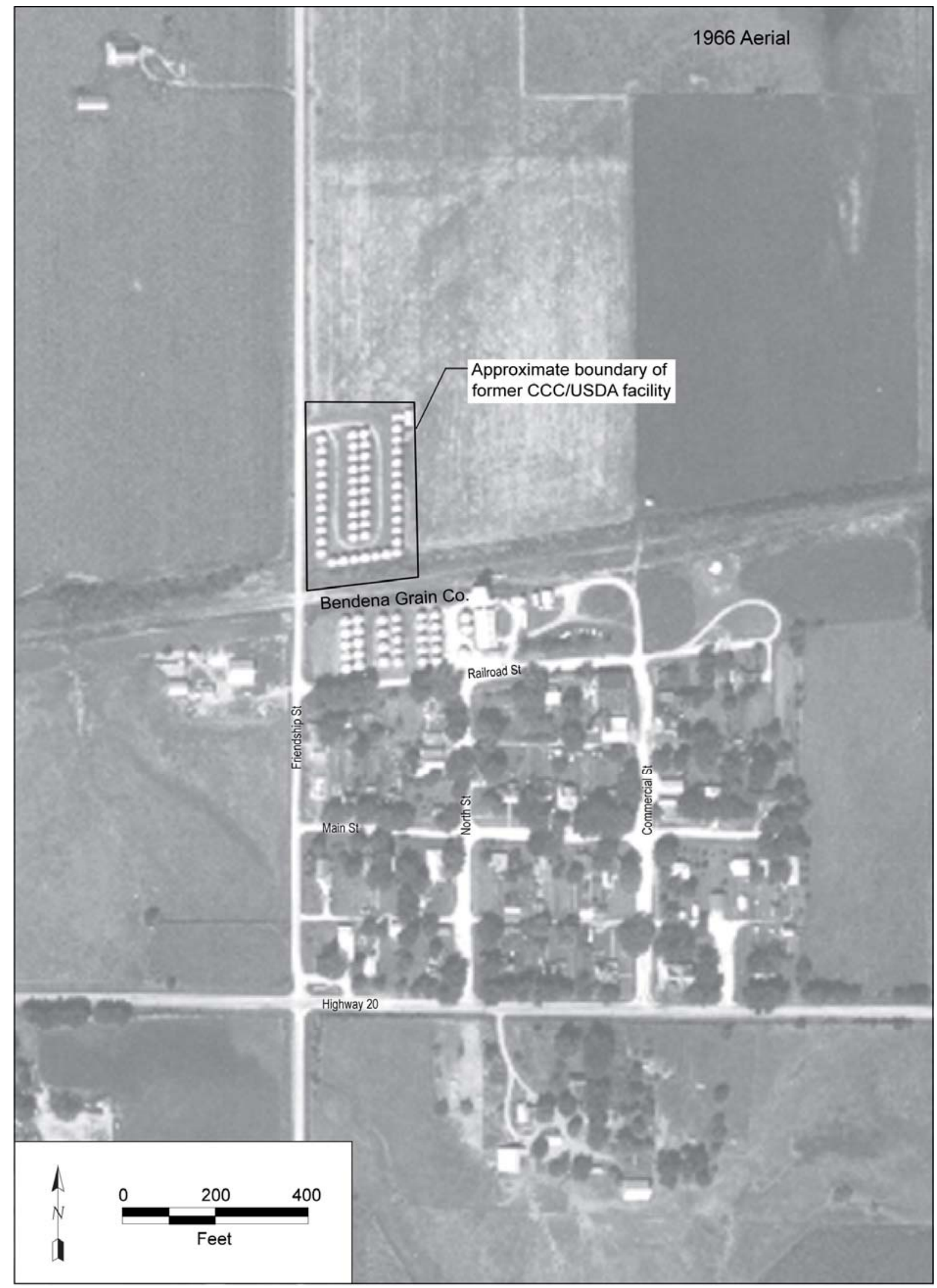

FIGURE 2.2 Locations of grain storage facilities in 1966. Source of photograph: USDA (1966). 


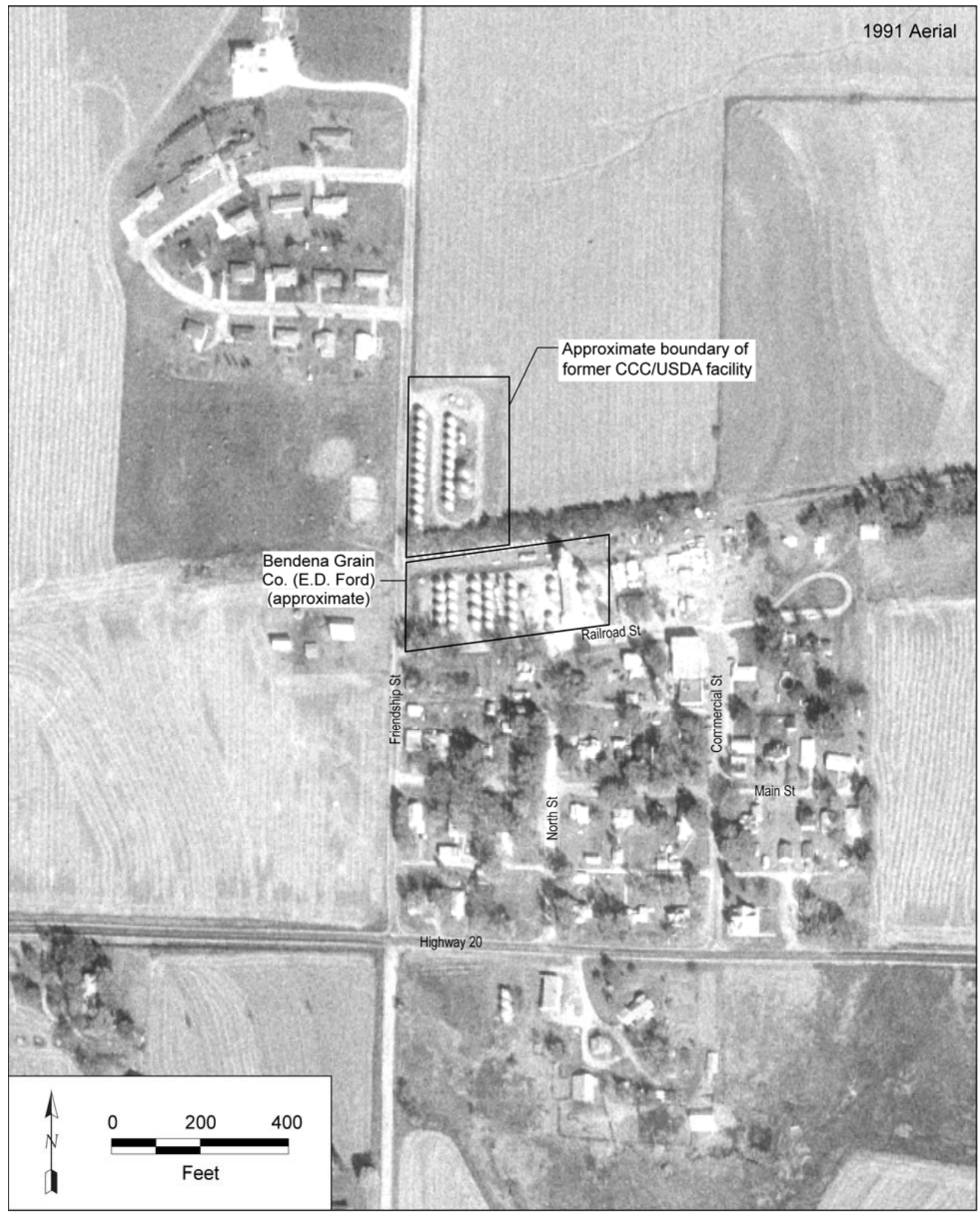

FIGURE 2.3 Locations of grain storage facilities in 1991. Source of photograph: USGS (1991). 


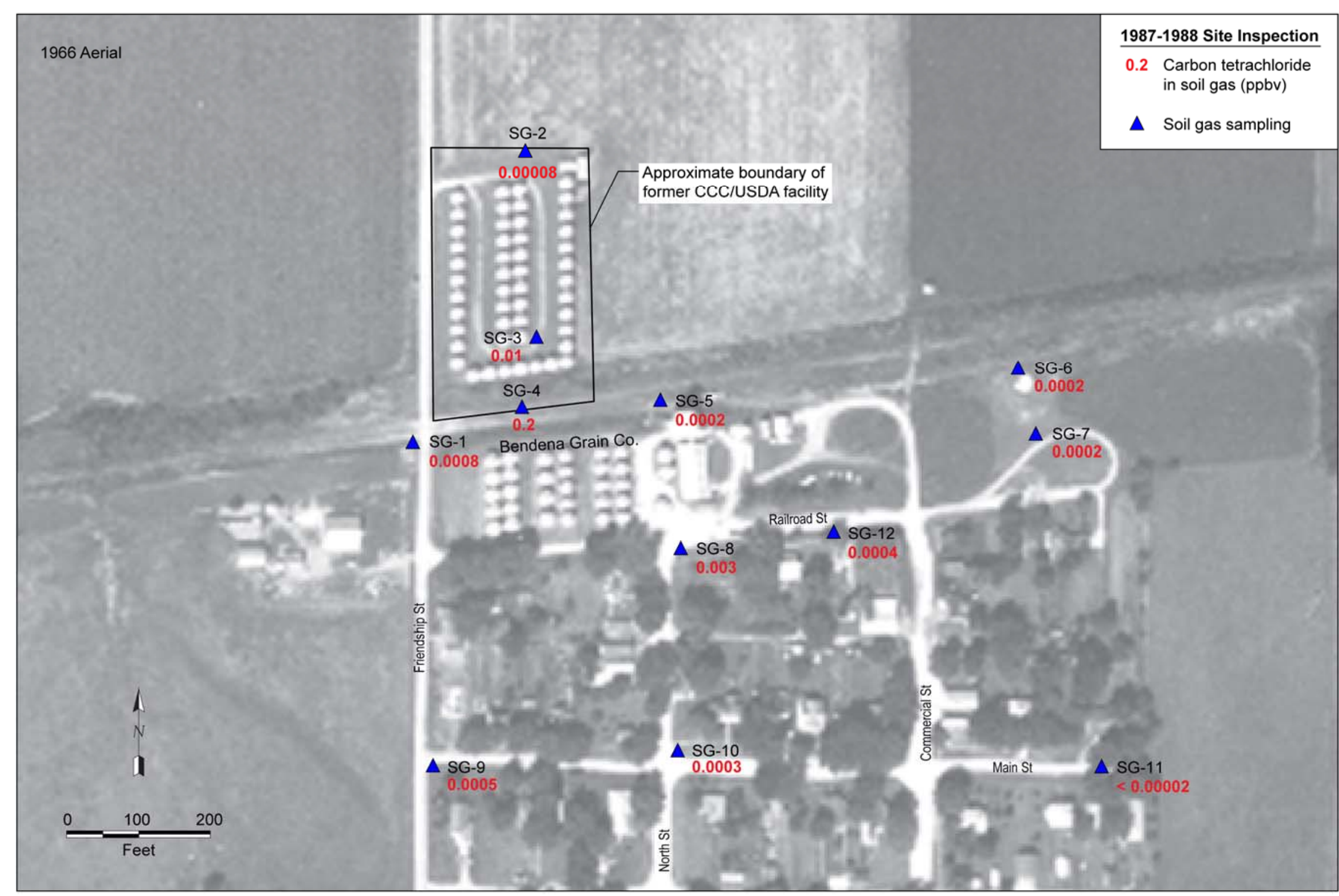

FIGURE 2.4 Sampling locations and results of analyses for carbon tetrachloride in soil gas samples collected during the 1987-1988 KDHE site inspection. Source of photograph: USDA (1966). 


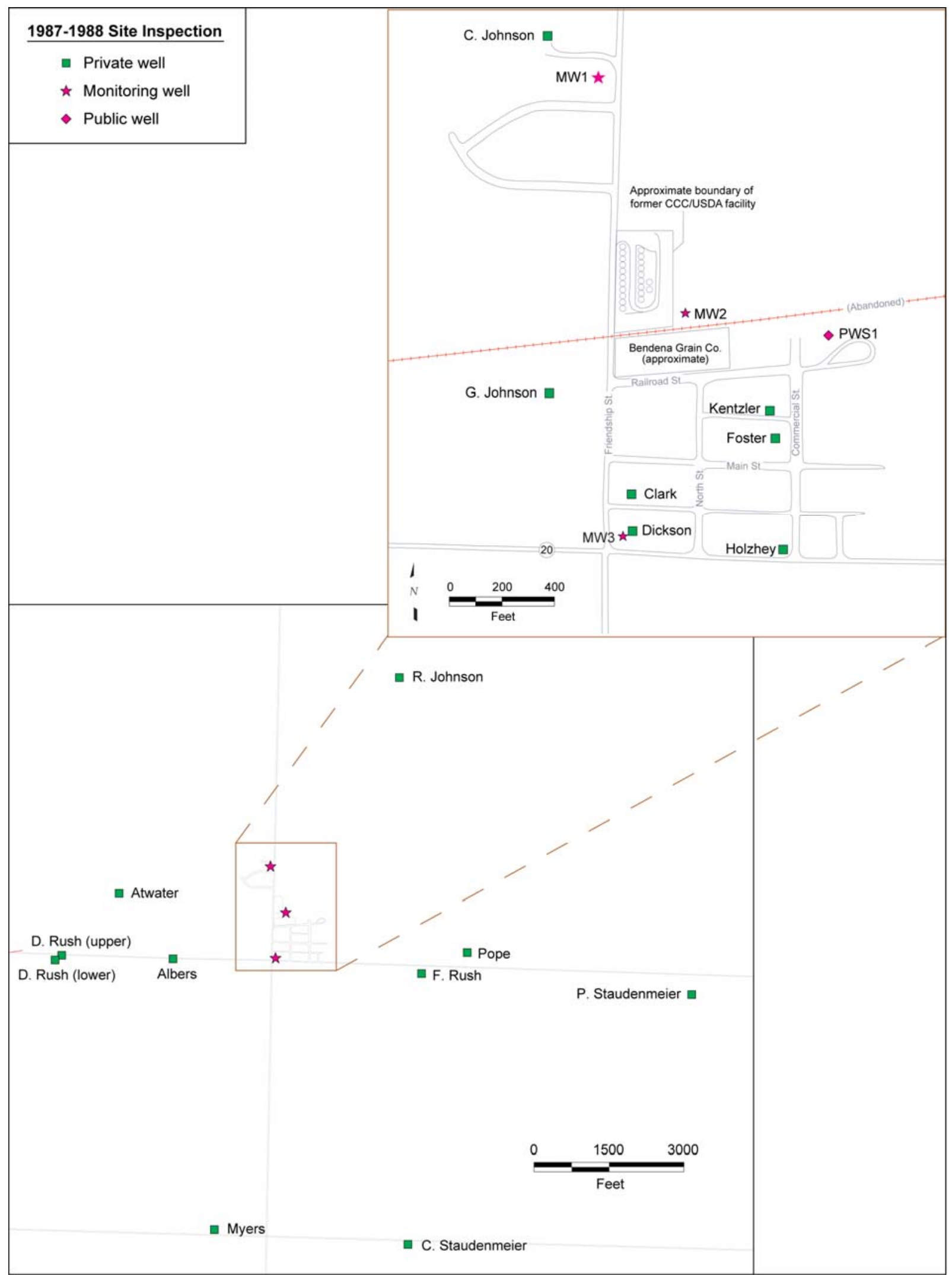

FIGURE 2.5 Locations of monitoring wells MW1-MW3 and private wells investigated during the 19871988 KDHE site inspection. 


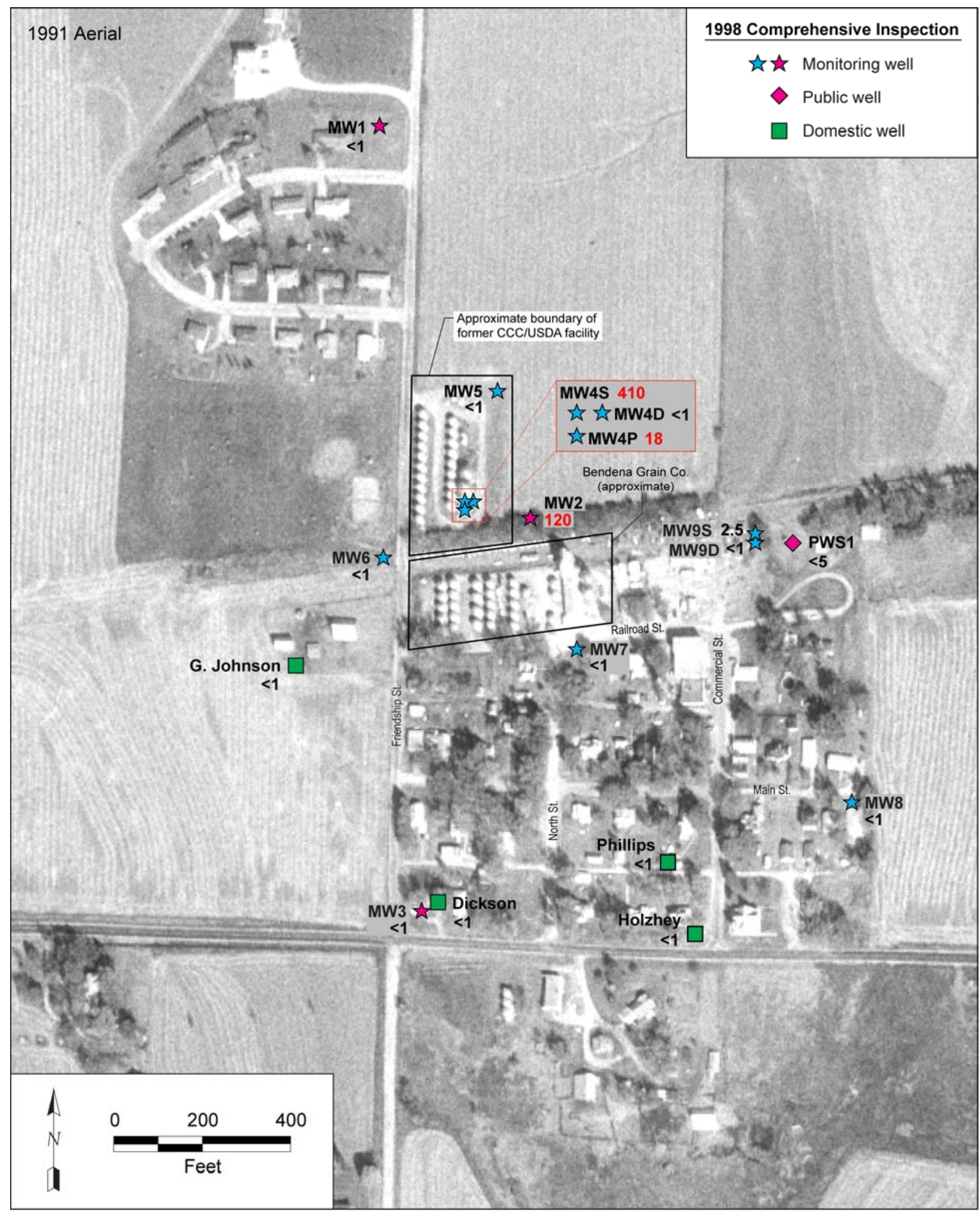

FIGURE 2.6 Sampling locations during the 1998 KDHE comprehensive investigation. Source of photograph: USGS (1991). 


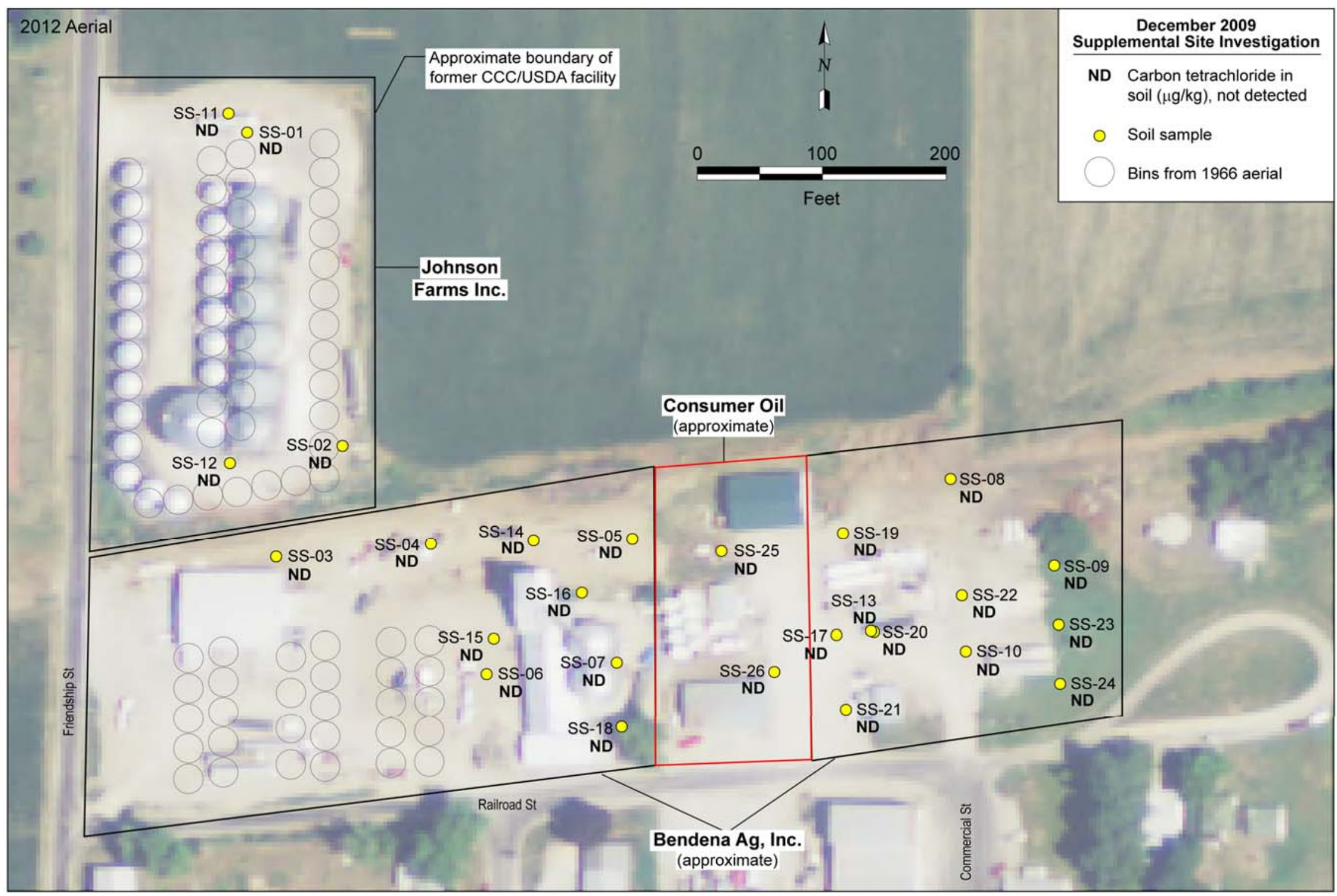

FIGURE 2.7 Shallow soil sampling locations in the 2009-2010 KDHE supplemental site investigation, with carbon tetrachloride results for the Argonne split soil samples. Source of photograph: NAIP (2012). 


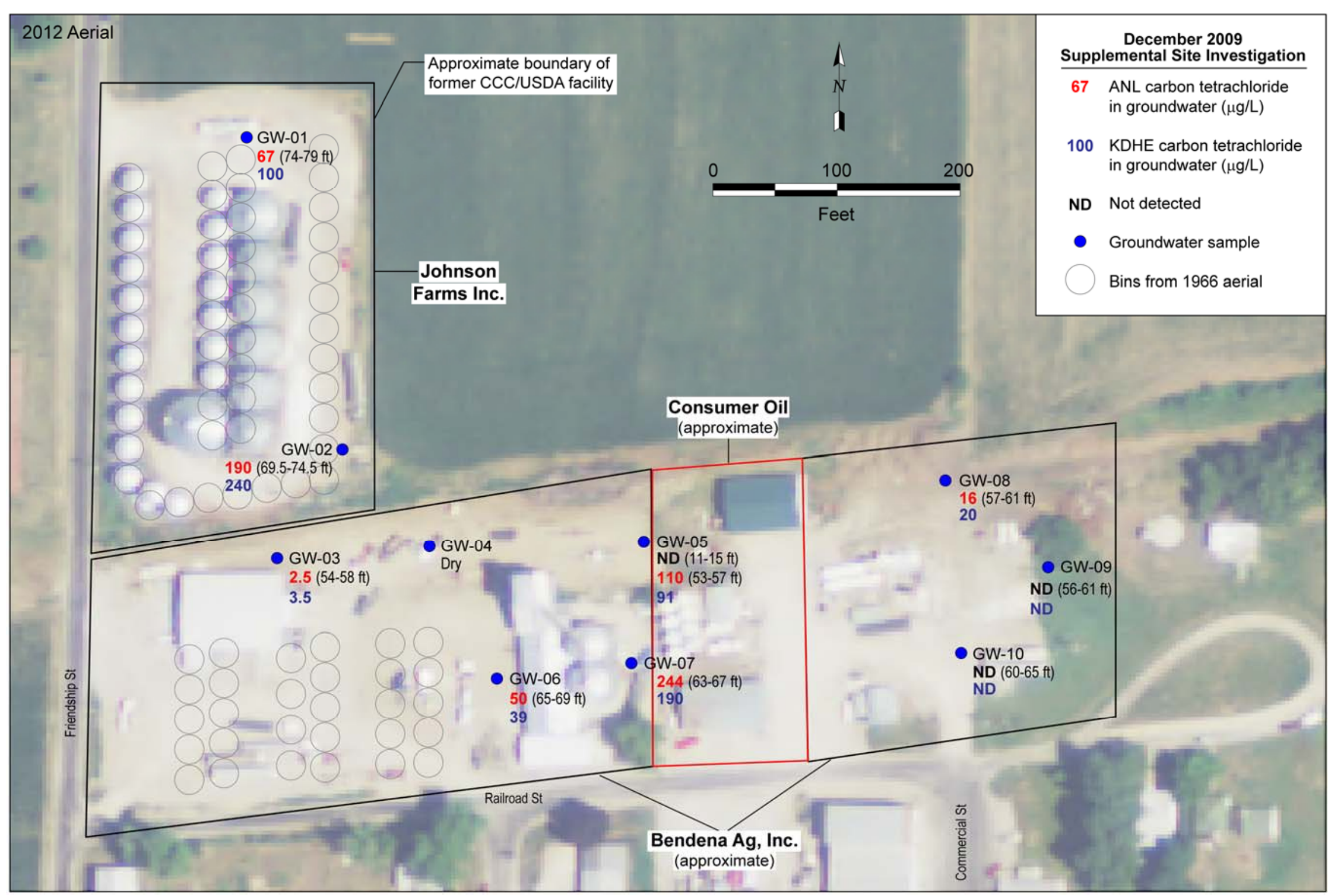

FIGURE 2.8 Groundwater sampling locations in the 2009-2010 KDHE supplemental site investigation, with carbon tetrachloride results for the KDHE and Argonne split groundwater samples. Source of photograph: NAIP (2012). 


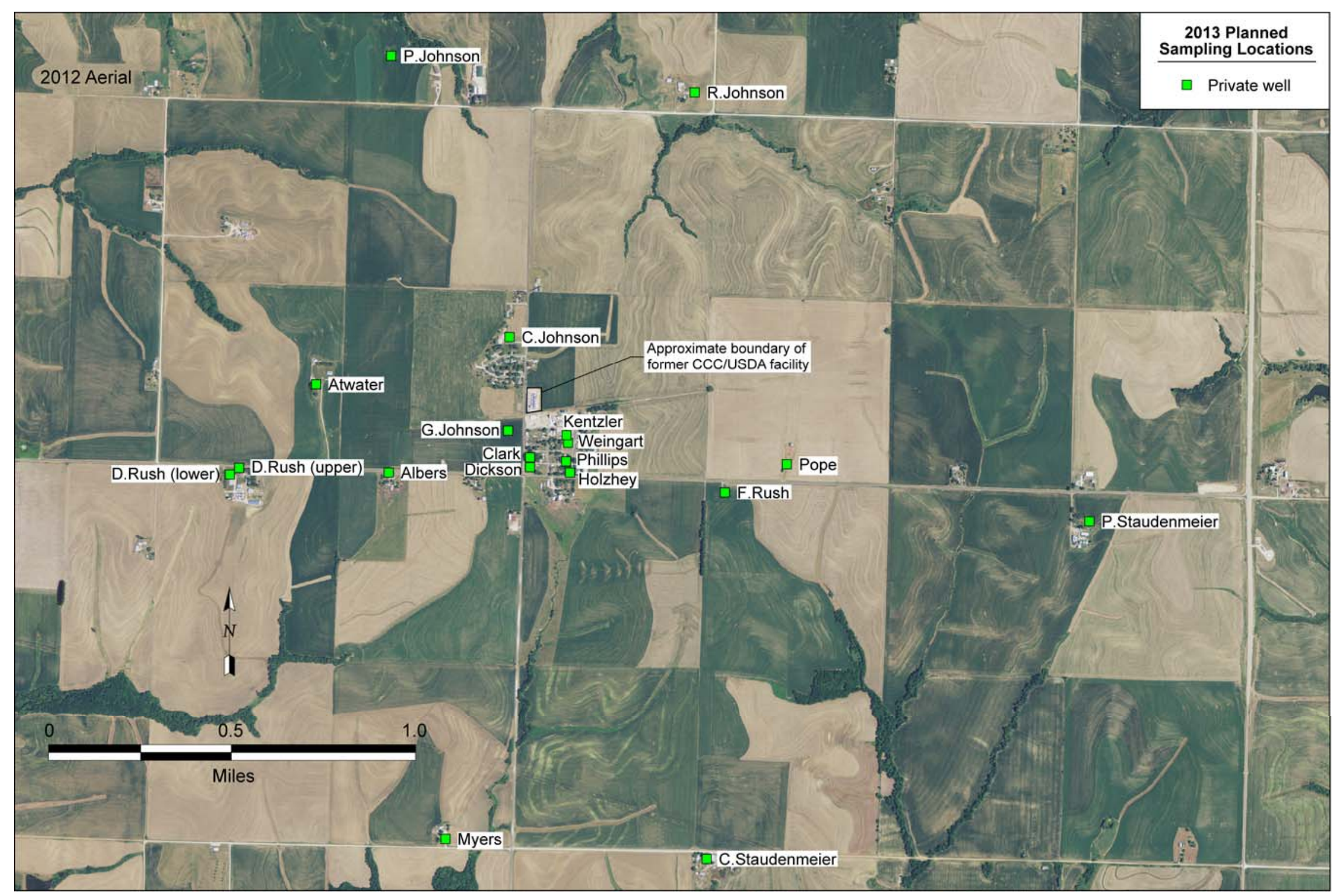




\begin{tabular}{|c|c|c|c|c|}
\hline Epoch & Stratigraphy & Formation or Group & Thickness & Physical Character \\
\hline \multirow{3}{*}{ 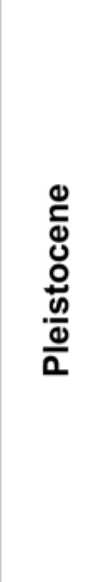 } & & $\begin{array}{l}\text { Alluvium and Terrace } \\
\text { Deposits }\end{array}$ & $0-120 \mathrm{ft}$ & $\begin{array}{l}\text { Clay, silt, sand, and gravel in principal stream valleys. } \\
\text { Coarser materials generally in lower portions of the } \\
\text { deposits. }\end{array}$ \\
\hline & & $\begin{array}{l}\text { Peoria, Gilman Canyon, } \\
\text { and Loveland Formations }\end{array}$ & 0 - $150 \mathrm{ft}$. & $\begin{array}{l}\text { Brown to reddish-brown silt and silty clay loess. } \\
\text { Generally } 10 \text { to } 30 \mathrm{ft} \text {. thick on uplands. Thickness } \\
\text { locally exceeds } 100 \mathrm{ft} \text {. along banks of the Missouri } \\
\text { river. }\end{array}$ \\
\hline & $\frac{0}{0+0}$ & $\begin{array}{l}\text { Independence Formation } \\
\text { (Till and outwash deposits) }\end{array}$ & $0-150 \mathrm{ft}$. & $\begin{array}{l}\text { Gray, blue-gray or brown terrigenous clay, containing wood } \\
\text { fragments, occasional to abundant limestone, quartzite } \\
\text { and other erratics, and lenses to sheet-like bodies of } \\
\text { stratified silt, sand and gravel. Abundance of sheet-like } \\
\text { bodies increases in southwestern portion of the county. }\end{array}$ \\
\hline \multirow{3}{*}{ 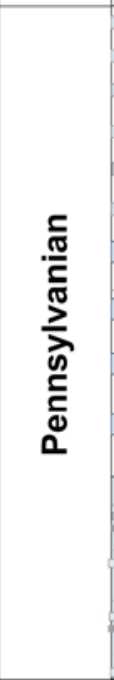 } & 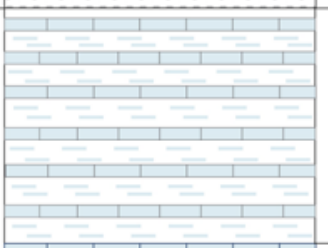 & Wabaunsee Group & $0-350 \mathrm{ft}$. & $\begin{array}{l}\text { Alternating thin beds of limestone and shale. } \\
\text { Contains some sandy shale and sandstone. }\end{array}$ \\
\hline & 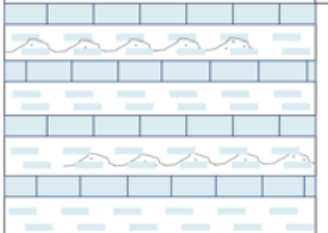 & Shawnee Group & $0-320 \mathrm{ft}$. & $\begin{array}{l}\text { Interbedded thick limestones and shales. } \\
\text { Shale contains some sandstone beds. }\end{array}$ \\
\hline & 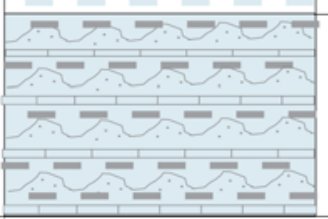 & Douglas Group & $0-250 \mathrm{ft}$. & $\begin{array}{l}\text { Shale, containing thick channel sandstones } \\
\text { and thin limestones. }\end{array}$ \\
\hline
\end{tabular}

FIGURE 2.10 Generalized stratigraphic column for the Doniphan County area. 


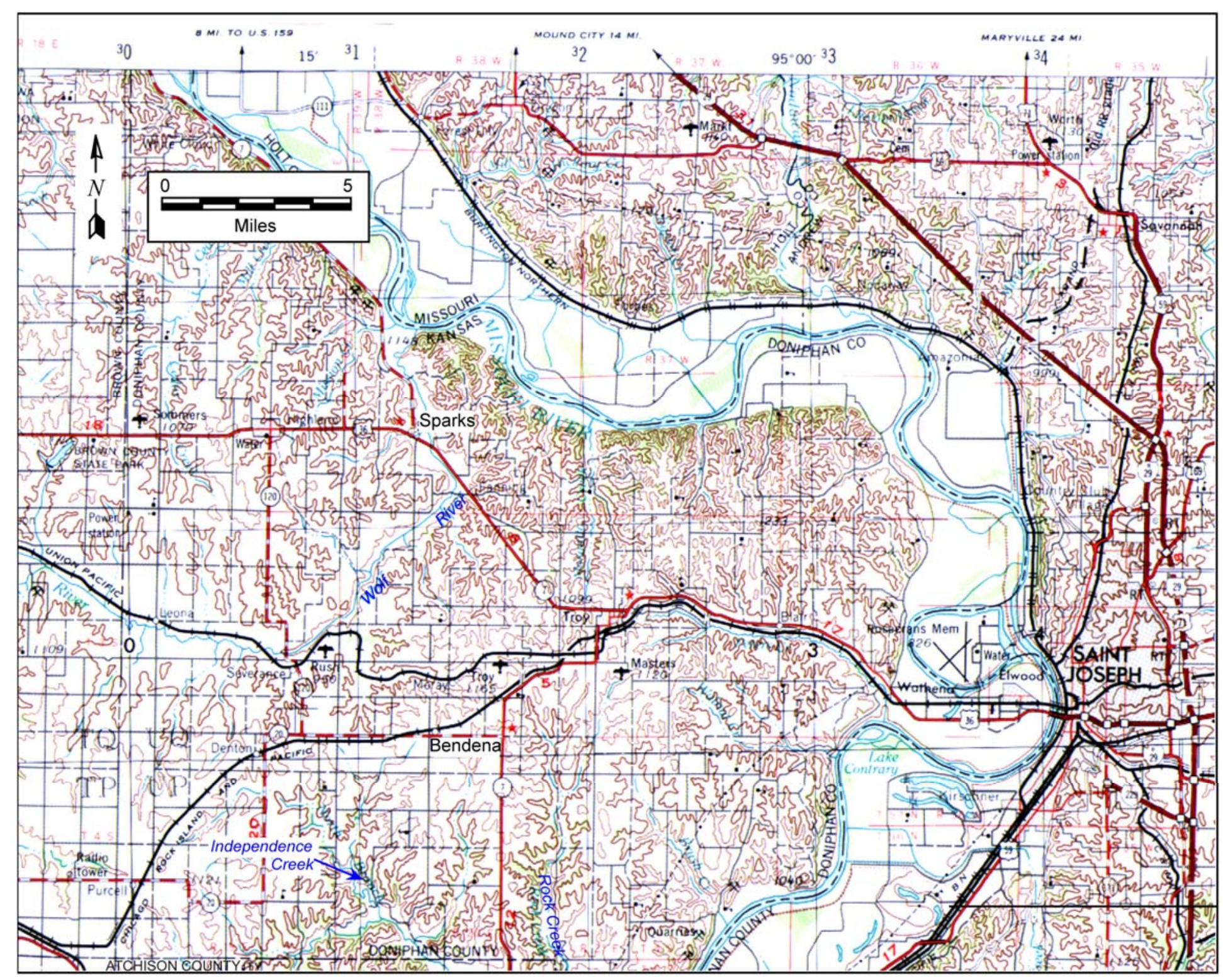

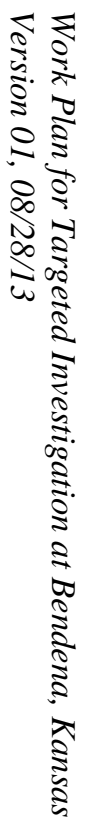

FIGURE 2.11 Modern topography of the Doniphan County Area. Source of map: USGS (1969). 


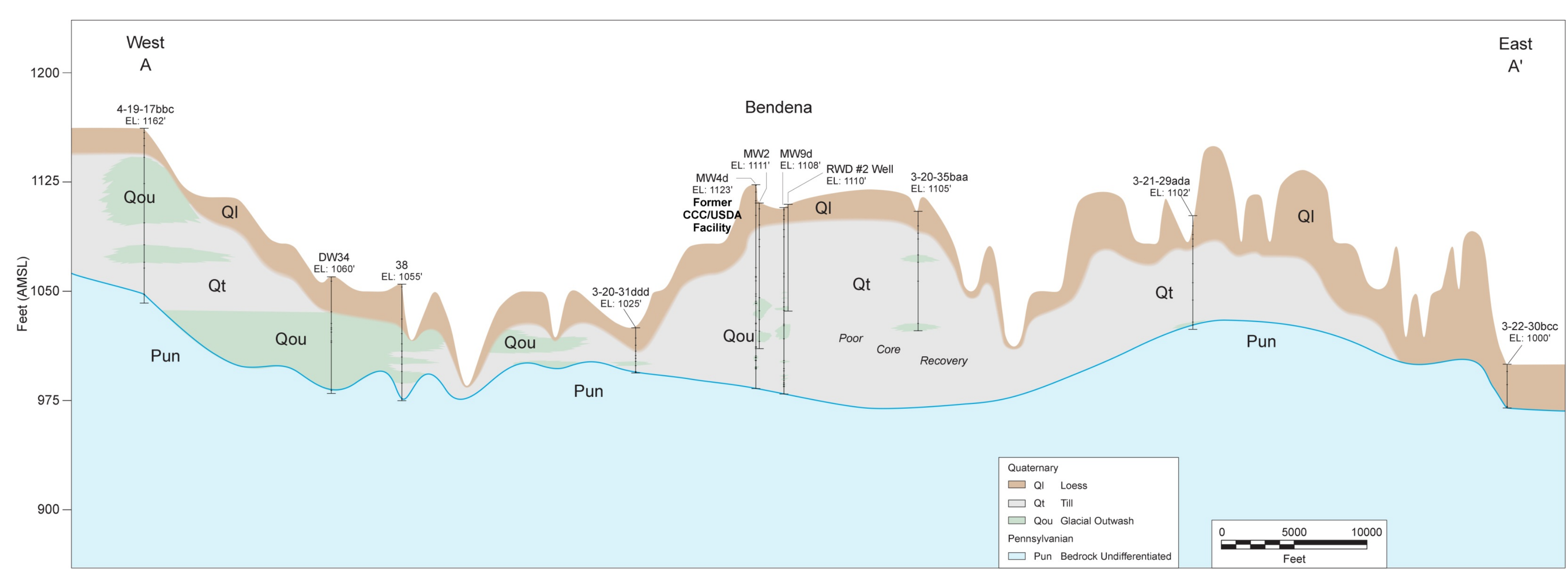

FIGURE 2.12 West-to-east geologic cross section A-A' (vertically exaggerated) across Doniphan County and through Bendena, at the location shown in Figure 2.13. 


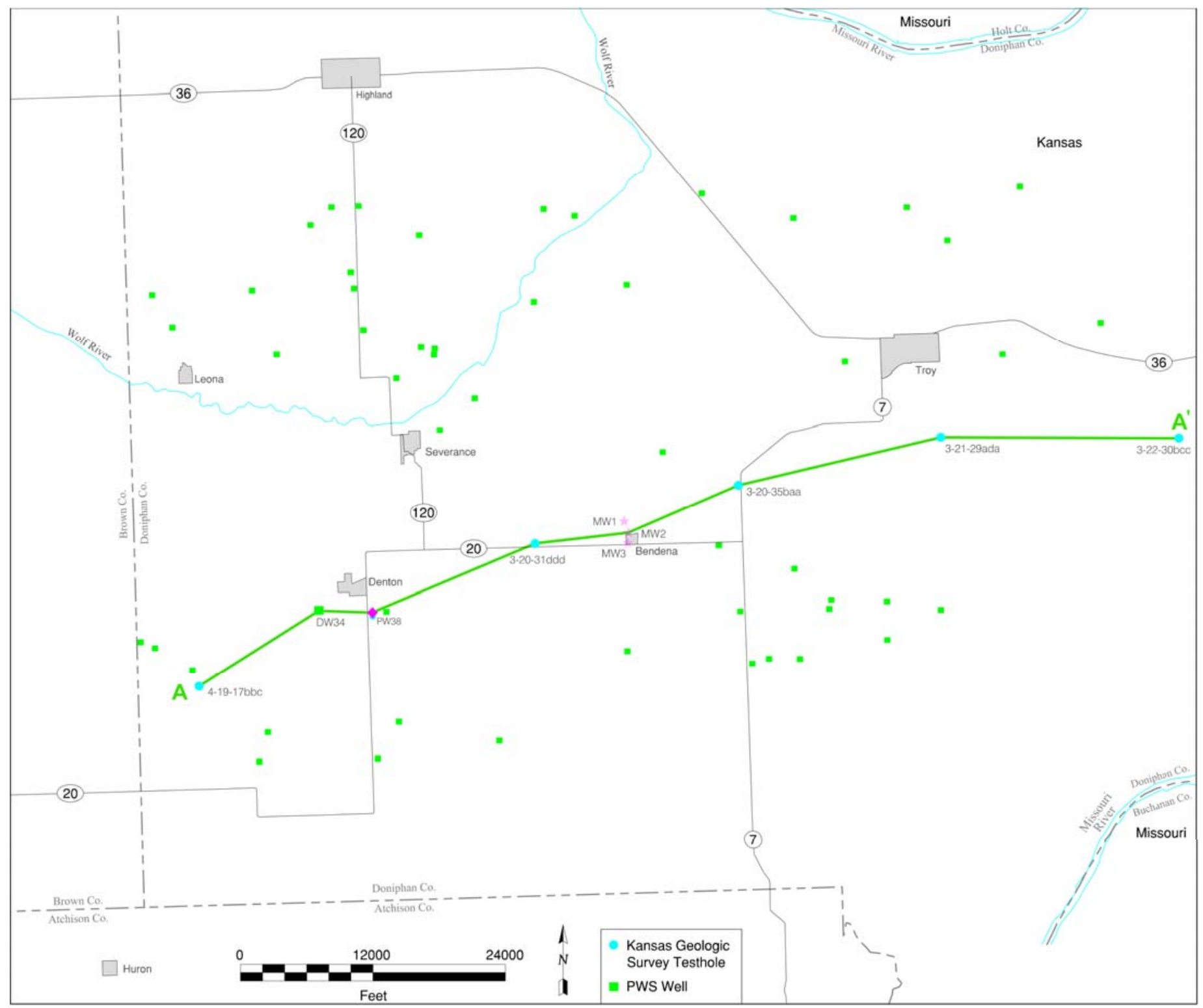

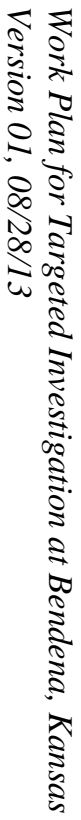

FIGURE 2.13 Location of west-to-east geologic cross section A-A' across Doniphan County 


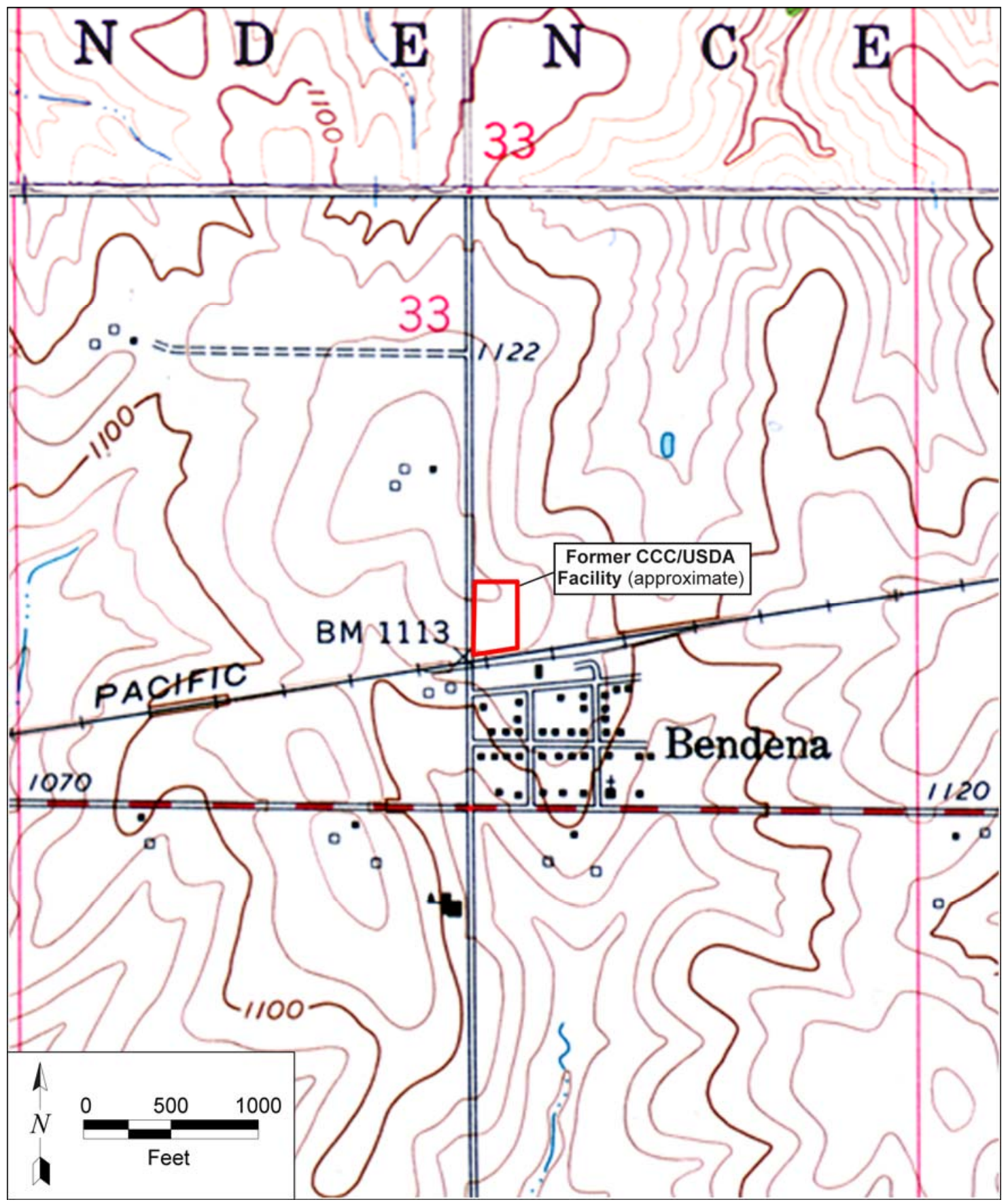

FIGURE 2.14 Local topography of the Bendena area. Source of map: USGS (1969). 


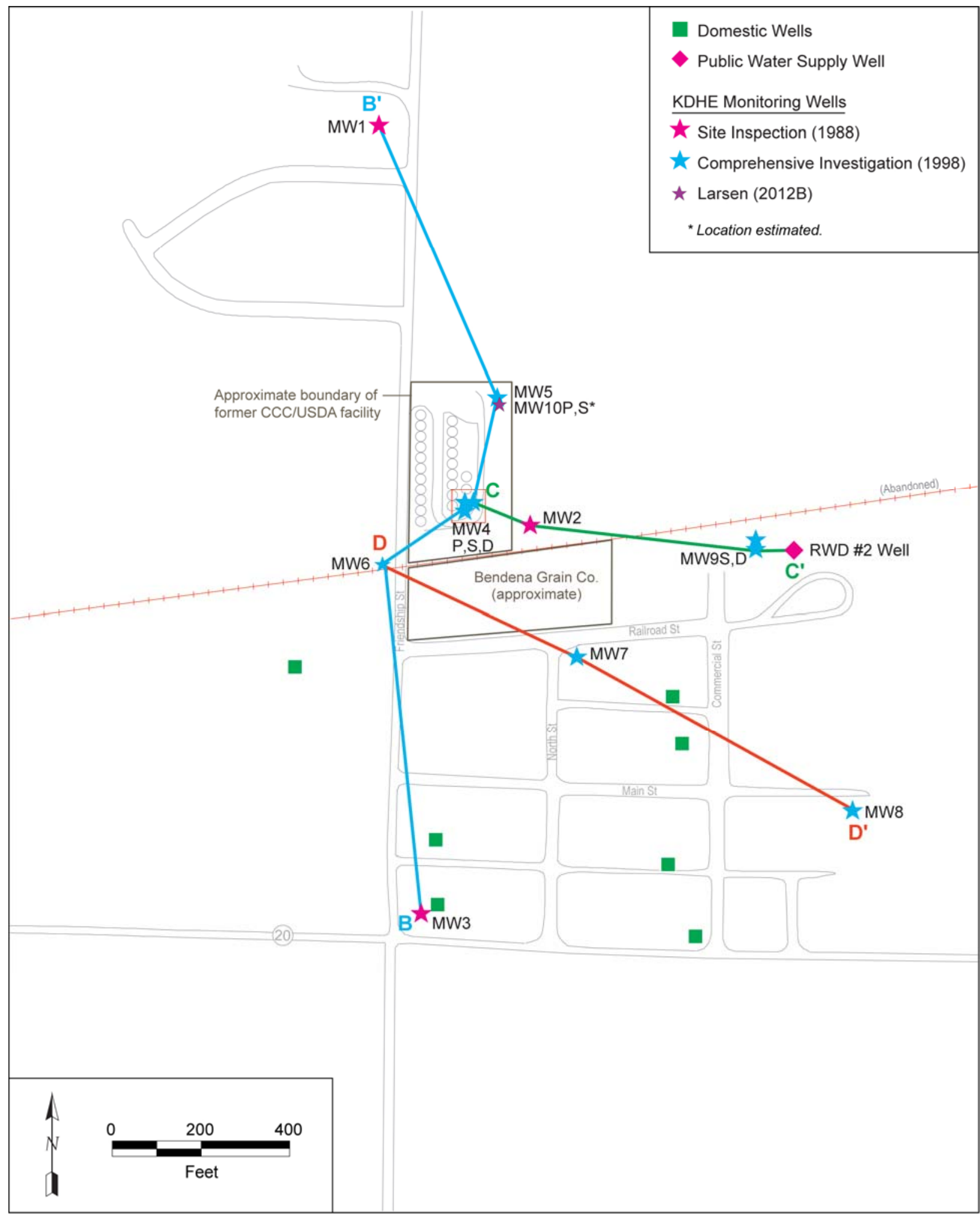

FIGURE 2.15 Locations of hydrogeologic cross sections B-B', C-C', and D-D' at Bendena, with public water supply, domestic, and monitoring wells. 


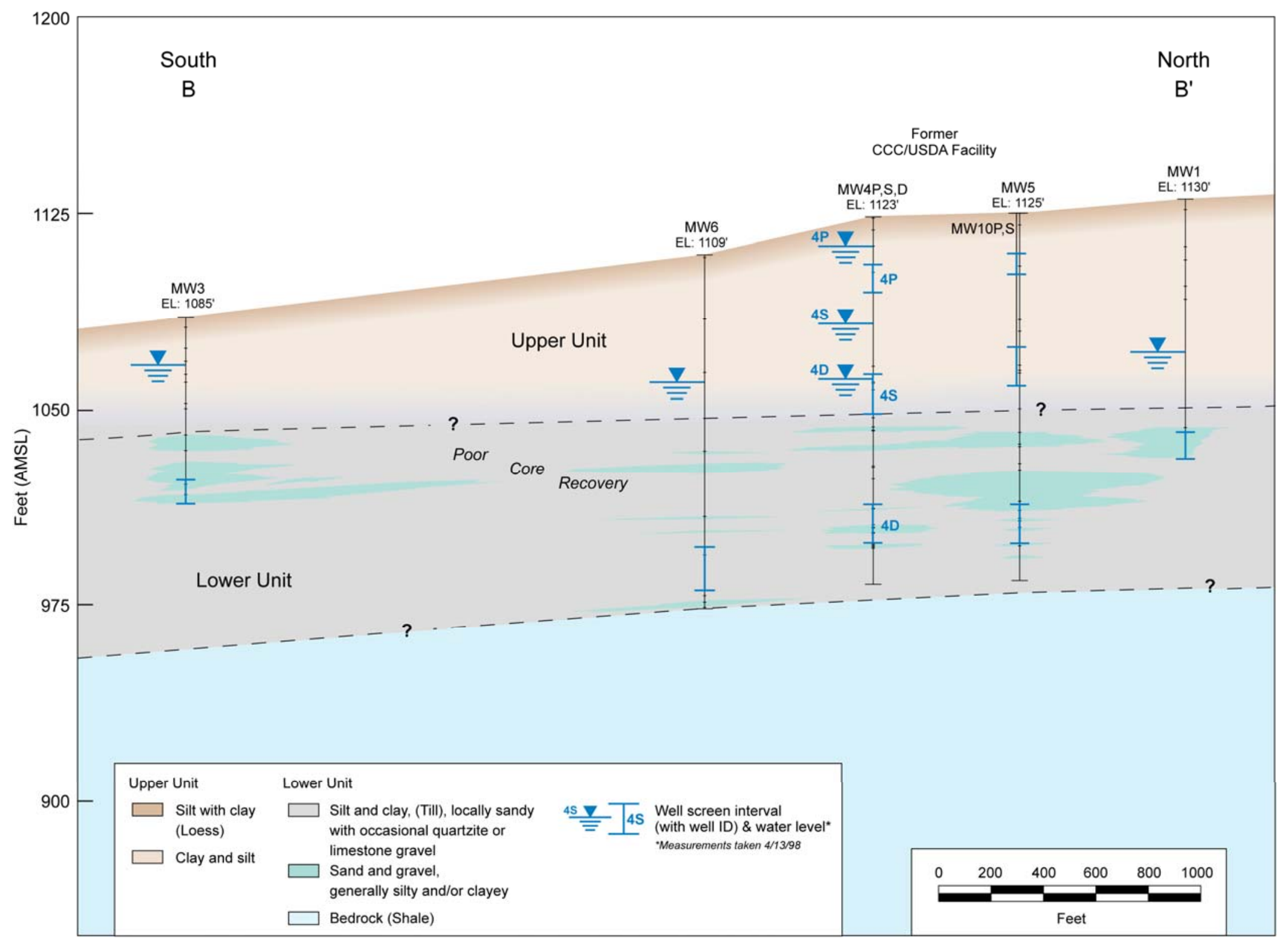

FIGURE 2.16 South-to north hydrogeologic cross section B-B' (vertically exaggerated) at Bendena. 


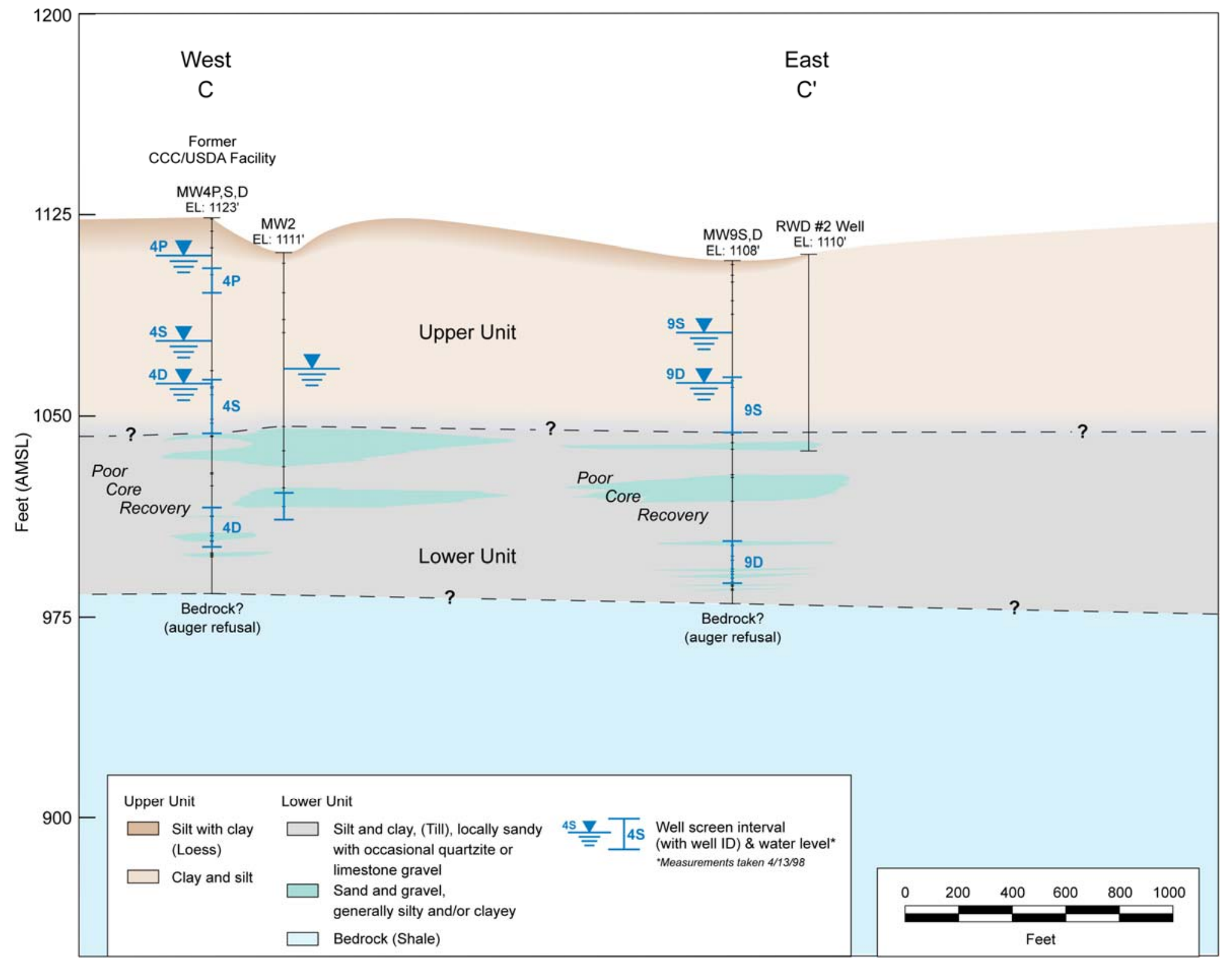




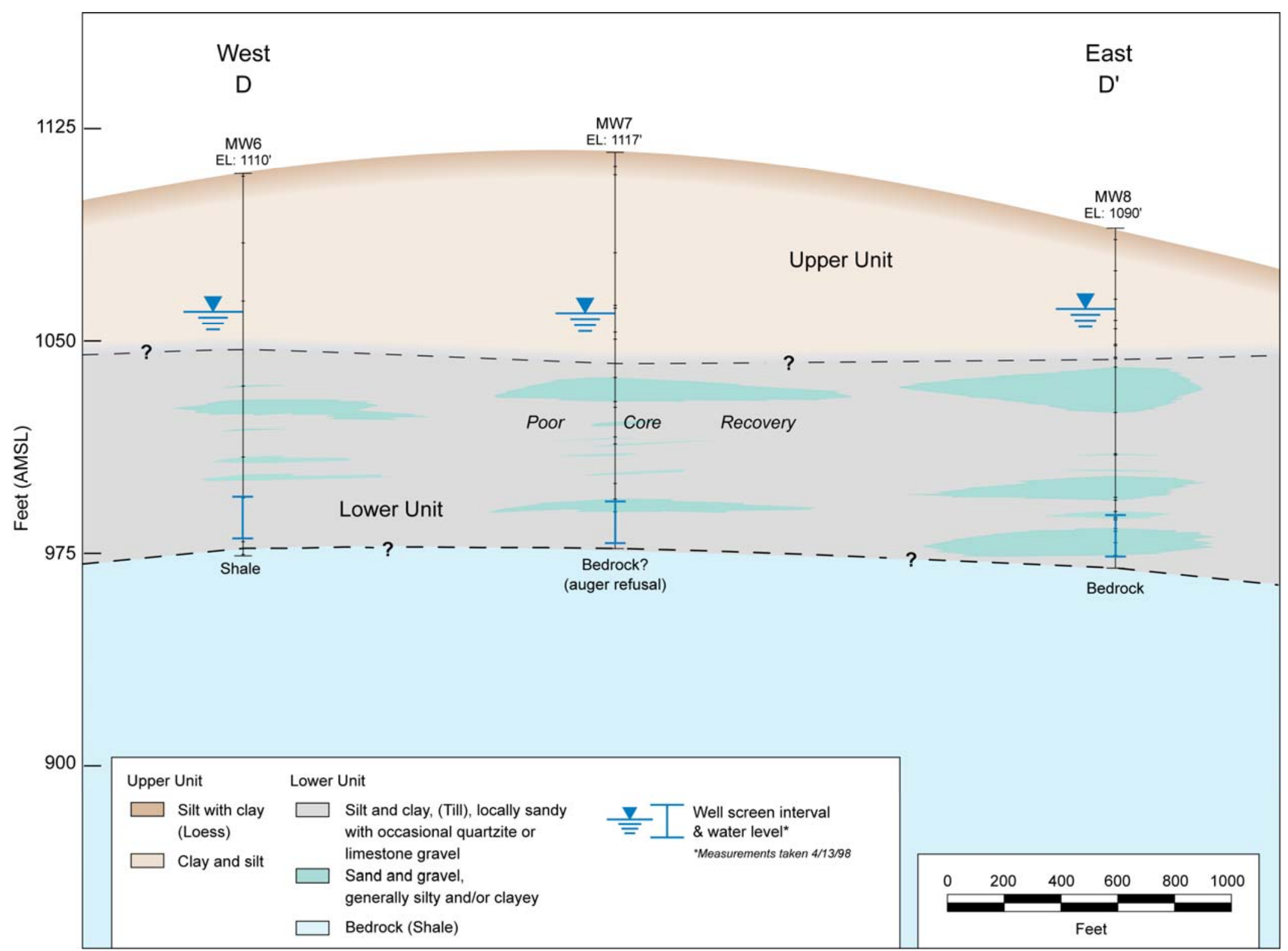




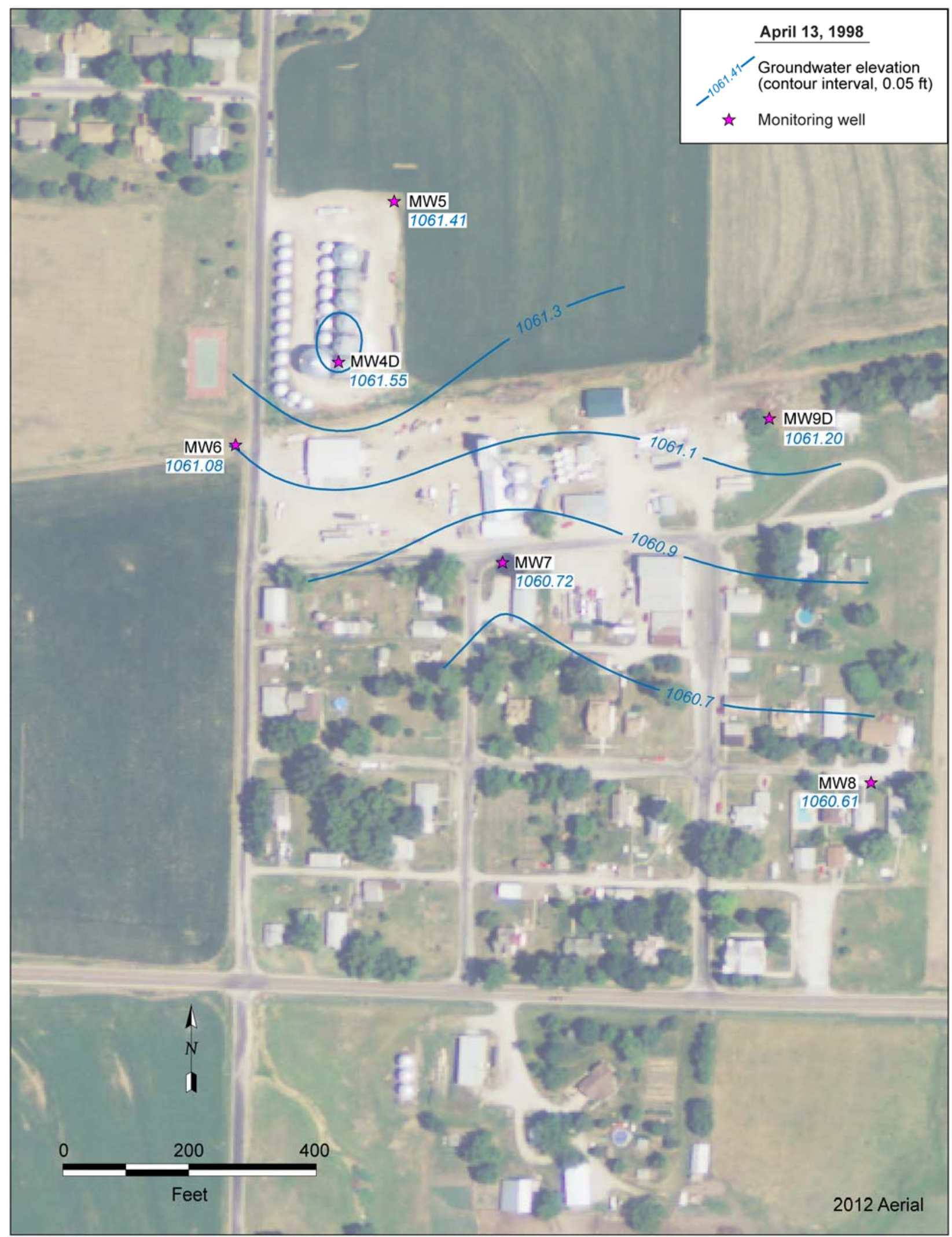

FIGURE 2.19 Mechanically contoured potentiometric surface for water levels measured in 1998 in the lower aquifer monitoring wells, including MW7. Source of photograph: NAIP (2012). 


\section{Proposed Technical Program for the Investigation}

The technical program presented in this section outlines the initial stage of the investigations to be conducted by the CCC/USDA at Bendena. The primary goal of this program is to determine the potential relationship between (1) the carbon tetrachloride contamination previously identified in soil and groundwater at the former CCC/USDA grain storage facility and (2) the carbon tetrachloride historically detected in groundwater at well PWS1, several permanent monitoring wells, and several investigative borings in the vicinity of the former CCC/USDA facility. The work at Bendena will be conducted in phases, in consultation with the CCC/USDA and KDHE project managers, so that the technical efforts at each stage of the investigation can be optimized by incorporating the results obtained earlier in the study.

The historical data evaluation summarized in Section 2, together with discussions among the KDHE, the CCC/USDA, and key property owners that affect critical site access at Bendena, provided the context for development of the Phase I technical program. In keeping with these factors, the present study is focused on investigation of (1) the vertical and lateral distribution of carbon tetrachloride and (2) the hydrogeologic factors affecting the movement of the contaminant beneath the former CCC/USDA facility (on property now owned by Johnson Farms, Inc.) and the adjacent properties to the south and southeast presently owned by Bendena Ag and Consumer Oil (Figure 3.1).

\subsection{Technical Objectives}

To guide this phase of the studies at Bendena, the following specific technical objectives are proposed:

- Investigate the carbon tetrachloride contamination previously identified in soils and groundwater; evaluate the former CCC/USDA facility (on property now owned by Johnson Farms) as a potential source of contamination to the local groundwater.

- Investigate the distribution of carbon tetrachloride contamination in the subsurface beneath the properties currently owned by Bendena Ag and the Consumer Oil Co., south and southeast of the former CCC/USDA facility. 
- Verify the lithologic and hydrologic characteristics of the unconsolidated geologic sequence hosting the groundwater flow system in the vicinity of the former CCC/USDA facility.

- Identify and document the patterns of groundwater flow and potential contaminant migration in the local hydrogeologic system.

- Update the inventory and status of local private wells; conduct limited private well sampling as appropriate, subject to access, to identify potential downgradient receptors.

\subsection{Primary Investigation Tasks}

To achieve these technical objectives, five primary investigation tasks will be addressed in the Phase I field program. The specific activities to be implemented under each task are summarized below. Argonne will review the results of each task as they are received in the field and will consult with the CCC/USDA and KDHE project managers to ensure that the program is meeting the stated technical objectives. To this end, the tasks and investigation locations recommended below, which were selected to accommodate these multiple objectives in a costand time-effective manner, will be subject to further review and possible modification (with the consent of the CCC/USDA and KDHE project managers) as the field activities progress.

Each task described addresses a unique element of the investigative program; however, the tasks do not necessarily define a strict sequence of activities. For logistic reasons and for efficiency in the field, components of one or more primary tasks might be implemented or be in progress at any time during the on-site investigation.

\subsubsection{Task 1. Conduct Vertical Profiling at the Former CCC/USDA Facility to Determine the Distribution of Carbon Tetrachloride Contamination in Soils and Groundwater}

As outlined in Section 2.2, limited previous sampling indicated the presence of carbon tetrachloride contamination in soils and groundwater at the former CCC/USDA facility (now the Johnson Farms property); however, the potential vertical and areal extent of the carbon tetrachloride in these media has not been investigated. To verify these occurrences and provide 
more detailed information on the subsurface distribution of the contaminant, soil and groundwater sampling for VOCs analyses will be performed through the vadose and saturated zone(s) at investigative boring locations 1-4 in Figure 3.1. At each of these locations, continuous soil cores will be collected from the ground surface to the top of bedrock, to establish the detailed lithologic and hydrostratigraphic sequence beneath the former CCC/USDA facility.

Groundwater samples will be collected for VOCs analysis from each identified zone of greater permeability that readily produces groundwater. Soil samples for VOCs analysis will be collected from the less permeable materials encountered, to facilitate examination of the complete vertical distribution of potential carbon tetrachloride contamination at each location. The soil and groundwater sampling will be performed in accord with the procedures in the Master Work Plan (Argonne 2002) and the methods specified in Section 3.3. On the basis of these results, potential additional sampling of the soils and/or groundwater at the former CCC/USDA facility, at locations and depths to be determined in the field, will be discussed, if warranted, with the CCC/USDA and KDHE program managers.

Presently available lithologic information for the former CCC/USDA facility and the surrounding area (Section 2.3) suggests that the coring and sampling activities outlined above can be completed by using the Argonne 22-ton, track-mounted cone penetrometer (CPT) unit. If initial efforts to penetrate the unconsolidated sequence to the approximate top of bedrock with the CPT prove unsuccessful, Argonne will consult with the CCC/USDA and KDHE program managers and will have arrangements in place for potential continuation of the field program by using a conventional (sonic) drilling rig.

\subsubsection{Task 2. Conduct Vertical Profiling for VOCs Analysis at Selected Locations on the Bendena Ag and Consumer Oil Properties, to Investigate the Vertical and Areal Distribution of Carbon Tetrachloride Contamination and Potential Contaminant Migration Pathways}

Carbon tetrachloride contamination has historically been detected in groundwater at a limited number of sampled locations and depths beneath portions of the Bendena Ag property (Section 2.2). To date, no groundwater sampling for VOCs analysis has been performed on the Consumer Oil property, and sampling on the Bendena Ag property has taken place in the absence of location-specific hydrogeologic data that might assist in the identification of potential discrete water-bearing intervals and/or preferred groundwater (contaminant) migration pathways. 
To address these concerns, vertical profiling — to include continuous soil coring and soil and groundwater sampling as outlined in Section 3.2.1 — will be conducted at investigative boring locations 5-11 in Figure 3.1. On the basis of the results from these borings, further sampling on the Bendena Ag and/or Consumer Oil properties might be warranted.

\subsubsection{Task 3. Determine the Lithologic and Hydrologic Characteristics of the Unconsolidated Units Hosting the Groundwater Flow System}

Existing lithologic and hydrologic data pertinent to the former CCC/USDA facility and the adjacent Bendena Ag and Consumer Oil properties are summarized in Section 2.3. On the basis of this information, three potential aquifer units have historically been interpreted to exist within a sequence of silty clays and heterogeneous silty to sandy or gravelly tills that underlie the area. The vertical and lateral extent and more detailed internal characteristics of these inferred units remain unclear, partly because of incomplete lithologic information at several previously investigated locations (Section 2.3.2).

As noted in Sections 3.2.1 and 3.2.2, continuous coring of the unconsolidated materials from the ground surface to bedrock will be performed to obtain a record of the subsurface geology and hydrogeology at investigative boring locations 1-11 on the former CCC/USDA, Bendena Ag, and Consumer Oil properties (Figure 3.1). The hydrogeologic sequences at these locations will be characterized in detail to determine the range of lithologies and types of sedimentary units present and to identify water-bearing and potential confining intervals. The sequences observed will also be compared to evaluate the potential vertical and lateral continuity of the hydrologic units beneath and near the former CCC/USDA facility.

At selected locations and depth intervals to be determined in the field, soil samples will also be collected for the measurement of physical properties including particle size distribution, moisture content, porosity, bulk density, and total organic carbon, to facilitate the possible future quantitative estimation of groundwater and carbon tetrachloride migration rates in the saturated zone(s). 


\subsubsection{Task 4. Install Permanent Groundwater Monitoring Points to Investigate the Patterns of Groundwater Movement}

At present, 11 permanent monitoring wells (MW4P,S,D; MW5-MW8, MW9S,D; MW10P,S; Figure 3.1) are distributed among 3 inferred aquifer units: the perched, shallow, and lower aquifers (KDHE 1998). Sporadic measurements of the groundwater levels in these wells (Table 2.6) suggest temporal variations in the groundwater levels across the site, as well as in the magnitude of potential vertical and lateral hydraulic gradients, that might affect groundwater flow in the saturated interval(s). The present network of monitoring points is insufficient, however, to determine the potential 3-dimensional patterns of groundwater flow in this system with confidence (Section 2.3.2.2).

To develop a more detailed understanding of the local groundwater flow, additional permanent monitoring points will be installed (by using the CPT or a sonic drilling rig) to augment the existing array of wells, at selected boring locations to be investigated in Phase I. On the basis of currently available information, the installation of approximately 9 new wells is proposed, as shown in Figure 3.2, to create vertically distinct networks of multiple wells in both the shallow and lower portions of the groundwater system; specific locations and depth intervals for the completion of monitoring wells will be determined in the field, however, subject to the findings of the continuous coring program as this information becomes available. If other potentially significant, vertically distinct water-bearing intervals (the perched aquifer or as-yetunidentified units) are encountered, these might also be considered for the installation of permanent monitoring wells.

Periodically, groundwater levels will be measured manually in the full suite of existing and new monitoring wells (at least $24 \mathrm{hr}$ after each well has been completed), to document the distribution of hydraulic heads in the local flow system. At selected monitoring points, automatic water level sensors and data loggers will also be installed to acquire continuous records of the water level fluctuations that might occur over a proposed minimum period of one year, to identify any short- or longer-term influences on the local levels and patterns of groundwater flow.

Elevation and location control required for evaluation of the potentiometric surface(s) within the system will be obtained via a professional survey (to be conducted by a licensed surveyor) of all the permanent monitoring points. 


\subsubsection{Task 5. Conduct Groundwater Sampling at the Permanent Monitoring Wells and Nearby Private Wells}

Groundwater samples for VOCs analysis will be collected from all available permanent monitoring points (previously existing and newly installed), following well completion and development and the stabilization of groundwater levels, to determine the relationship of the potential carbon tetrachloride concentrations detected in these wells to the concentrations identified at the vertical-profile sampling locations described in Sections 3.2.1 and 3.2.2.

As outlined in Section 2.2, groundwater sampling has been performed periodically in selected private wells near the former CCC/USDA facility and the Bendena Ag and Consumer Oil properties, most recently in 2013 (KDHE 2012). As part of the Phase I studies, the locations, status, and current ownership and usage of identifiable private wells within the town limits and/or in the immediate vicinity of the above properties will be determined. Subject to access, any identified private wells potentially at risk that were not sampled during the KDHE 2013 sampling event will be sampled during Phase I.

The results of this task will provide a self-consistent set of groundwater data for all of the accessible permanent groundwater monitoring points in the Bendena study area, to serve as a current baseline for the consideration and evaluation of potential future sampling event(s).

\subsection{Investigation Methods}

\subsubsection{Methods for Collecting and Logging Soil Cores}

At each boring location identified in Sections 3.2.1 and 3.2.2, core samples will be obtained in a continuous profile from approximately $2 \mathrm{ft}$ BGL to the top of bedrock (or refusal of the CPT coring equipment). The cores will be collected by using components of the GeoProbe ${ }^{\mathrm{TM}}$ dual-tube or Macro-Core coring systems that have been adapted for use with the Argonne CPT vehicle, or through the use of a sonic drilling rig (if sufficient penetration cannot be achieved with the CPT). All cores will be logged in accord with procedures in the Master Work Plan (Argonne 2002) and will be archived upon completion of the Phase I field program at an Argonne facility for future reference. 


\subsubsection{Methods for Sampling and VOCs Analyses of Soils}

At each boring location discussed in Sections 3.2.1 and 3.2.2, soil samples for VOCs analysis will be collected at approximately 4-ft intervals in the finer-grained (silty-clayey) units identified in the stratigraphic sequence. Samples from additional depths in the cored intervals at each boring might also be collected, as necessary, to adequately represent significant variations observed in the lithologies penetrated.

Soil samples for the determination of VOCs will be placed immediately in jars, sealed, and preserved on dry ice, then shipped overnight to the Applied Geosciences and Environmental Management (AGEM) Laboratory at Argonne for rapid-turnaround (typically 24-hr) analysis, so that interim results can be evaluated in the field as the sampling progresses. The soil samples will be analyzed first by a headspace method with a gas chromatograph and electron capture detector (modified EPA Method 5021), on a rapid-turnaround basis. The soil samples will also be subjected to purge-and-trap sample preparation with analysis on a gas chromatograph-mass spectrometer system (EPA Methods 5030B and 8260 B), as described in the Master Work Plan (Argonne 2002).

\subsubsection{Methods for Groundwater Sampling}

Groundwater sampling from the investigative borings (Sections 3.2.1 and 3.2.2) will follow the procedures in the Master Work Plan (Argonne 2002).

For groundwater sampling with the CPT, the CPT rods will be used to push a sacrificial tip and 0.5-in.-I.D. polyvinyl chloride (PVC) filter screen and riser to the desired maximum sampling depth. The rods will then be partially withdrawn (a maximum distance of $5 \mathrm{ft}$ ) to the desired minimum sampling depth, to expose the screen to the formation waters. Samples will then be recovered from the PVC casing by using a bailer, without purging.

If the use of a sonic drilling rig is required to achieve the Phase I objectives, groundwater samples will be collected by advancing the inner drill string and an outer override casing to the targeted sampling depth, where a grab sample will be collected. Alternatively, if the aquifer produces sufficient water ( $2 \mathrm{gpm}$ or more), then the inner drill sting will be removed, and a stainless steel screen and inflatable packer assembly will be advanced into the boring. The outer sonic casing will be vibrated upward the desired distance (no more than $5 \mathrm{ft}$ ) to expose the 
screen, and the packer will be inflated to isolate the screened zone. After purging of a minimum of three times the volume of the isolated sampling zone, the groundwater sample will be collected.

The samples will be collected in laboratory-approved containers and immediately placed in a cooler at $4^{\circ} \mathrm{C}$, pending shipment and analysis for VOCs as described in Section 3.3.5.

\subsubsection{Methods for Sampling of the Monitoring and Private Wells}

Water level measurements and sampling of the accessible private wells and all monitoring wells will be conducted in accord with the procedures outlined in the Master Work Plan (Argonne 2002), as follows:

- The well number, the well owner's name, or both will be recorded, with any additional relevant information or observations obtained at the time of sampling.

- If possible, the static groundwater level and then the total depth will be measured and documented for each well.

- Each well will be purged before it is sampled. For functional private wells, the existing, dedicated pump will be used for purging. For monitoring wells and all other private wells, a submersible pump provided by Argonne (RediFlo or similar; subject to physical access and the permission of individual well owners) will be used for purging. Groundwater from each well will be purged until field parameters of $\mathrm{pH}$, temperature, conductivity, oxidationreduction potential (ORP), and dissolved oxygen (DO) are stable. If possible, a minimum of three well volumes will be purged. The field parameters and volume purged will be documented.

- The wells will be sampled after adequate recharge has occurred, but no more than $24 \mathrm{hr}$ after purging. 
- Groundwater samples will be collected in laboratory-approved containers and placed immediately in a cooler at $4^{\circ} \mathrm{C}$, pending shipment and analysis for VOCs as described in Section 3.3.5.

\subsubsection{Methods for Analysis of Water Samples for VOCs}

Water samples for the determination of VOCs will be preserved and shipped overnight, on ice at $4^{\circ} \mathrm{C}$, to the AGEM Laboratory at Argonne for purge-and-trap sample preparation and analysis on a gas chromatograph-mass spectrometer system (EPA Methods 5030B and 8260B). To ensure reproducibility, a minimum of $10 \%$ of the water samples (to be selected in the field) will also be sent to a second laboratory (TestAmerica, Inc., South Burlington, Vermont) for verification analysis with the EPA's Contract Laboratory Program methods, in accord with the procedures in the Master Work Plan (Argonne 2002).

\subsubsection{Methods for the Installation of Monitoring Wells}

Monitoring wells will be installed according to the general procedures in Sections 6.4.3-6.4.6 of the Master Work Plan (Argonne 2002). The wells will be constructed by using nominal 1-in.-diameter (for wells installed with the CPT) or 2-in.-diameter (for drilled wells) PVC casing and 0.01-in. mill slot PVC screen of appropriate length for the desired interval and depth of completion. A 10/20 (or \#20) filter pack will be used, extending approximately $2 \mathrm{ft}$ above the screened interval. A bentonite pellet seal will be installed above the filter pack, and the remaining annulus will be grouted to the surface by using a tremie pipe.

All wells will be constructed in accordance with KDHE guidelines. Surface completions will consist of KDHE-approved flush mounts or aboveground housings, per the request of the property owner(s) for each well location. After installation, each well will be developed in accordance with the Master Work Plan (Argonne 2002).

\subsubsection{Methods for Handling and Disposal of Investigation-Derived Waste}

The approach to handling and disposal of waste soil and water is as follows: 
- Soil cores collected during sampling will be retained in core boxes for lithologic description and research. The cores will be transported to and stored at an Argonne facility for further reference.

- Waste soil from drilling activities (if generated) will be stored on-site in 55-gal drums or a roll-off container. A representative sample will be collected and analyzed by a KDHE-certified laboratory.

- A Solid Waste Disposal Authorization will be obtained from the KDHE for disposal of soil in a permitted landfill. If analytical data indicate that the soils cannot be placed in a permitted landfill, alternative disposal methods will be proposed to the KDHE for review.

- Wastewater will be stored on-site in 55-gal drums or polyurethane containers. If acceptable to the KDHE, the wastewater will be aerated prior to sampling and analysis for VOCs and nitrate.

- The results of the wastewater analyses will be discussed with the KDHE program manager, and an appropriate disposal method will be determined.

\subsection{Sampling and Reporting Schedule}

The proposed investigation is planned for fall 2013. The CCC/USDA and Argonne will notify the KDHE, Johnson Farms, Bendena Ag, and Consumer Oil a minimum of two weeks before the proposed field activities begin.

A report will be completed and submitted to the KDHE within 90 days upon completion of the investigation and after Argonne completes its quality review of the investigational data. The report will follow the guidelines for site monitoring established by KDHE Policy BER-RS036 (KDHE 2005). Accordingly, the report will include, at minimum, the following:

- A narrative of work conducted

- Recommendations for further action(s) at the site, if warranted 
- Maps depicting sample locations, groundwater flow directions(s), and contaminant levels

- Tables that include all analytical and field data

- Laboratory analytical data reports

- All relevant field documentation

- Quality assurance and quality control data

\subsection{Quality Assurance and Quality Control}

Procedures necessary to maintain the quality of data will be implemented throughout the proposed investigation. Descriptions of the quality assurance and quality control methods are in Section 4 of the Master Work Plan (Argonne 2002).

\subsection{Health and Safety}

A site-specific health and safety plan will be prepared, approved by the Argonne field safety coordinator, and brought to the site for reference during the investigation.

An Argonne health-safety-environmental protection representative will visit the site during field activities to observe, monitor, and report on operations.

The general health and safety plan for use during this project, which is in Section 3 of the Master Work Plan (Argonne 2002), addresses all anticipated safety issues for activities at the Bendena site. Specific emergency information for use at the site is given below.

Doniphan County has emergency 911 service. All emergency calls, including police, fire, and ambulance calls, will be directed for an appropriate response from this number. No medical facilities exist at Bendena. The nearest hospital with emergency medical facilities is in 
Atchison, Kansas (approximately $15 \mathrm{mi}$ from Bendena). Driving directions to the hospital are in Figure 3.3. Additional emergency information is in Table 3.1.

\subsection{Contacts}

\subsubsection{Johnson Farms Contacts}

President

Doug Johnson

Johnson Farms, Inc.

930 170th Road

Bendena, KS 66008

jfinc@rainbowtel.net

785-988-4321 (office)

KDHE Program Manager

Deanna Ross, PG

Remedial Section/Voluntary Cleanup

Bureau of Environmental Remediation

Kansas Department of Health and Environment

1000 SW Jackson Street, Suite 410

Topeka, KS 66612-1367

dross@kdheks.gov

785-296-8064 (office)
Consultant

Lisa Larsen, PG

Larsen \& Associates, Inc.

1311 E. 25th Street, Suite B

Lawrence, KS 66046

Lisa@LarsenEnvironmental.com

785-841-8707 (office)

785-331-9162 (cell)

KDHE Project Manager

Andrea Schiller

Remedial Section/Voluntary Cleanup

Bureau of Environmental Remediation

Kansas Department of Health and Environment

1000 SW Jackson Street, Suite 410

Topeka, KS 66612-1367

aschiller@kdheks.gov

785-296-0489 (office)

\subsubsection{Bendena Ag Contacts}

\section{Owner}

Patrick W. Urban

Bendena Ag, Inc.

202 Railroad Street

Bendena, KS 66008

785-988-4271 (office)
Legal Counsel

Edward L. Robinson

Joseph \& Hollander, LLC

500 North Market

Wichita, KS 67214

erobinson@josephhollander.com

316-262-9393 (office)

\section{Consultant}

Brian M. Conrad

Geotechnical Services, Inc.

435 Westport Road, Suite 23

Kansas City, MO 64111

bconrad@gsinetwork.com

816-561-1061 EXT. 151 (office)

913-558-2464 (cell) 


\subsubsection{Consumer Oil Contact}

President

Ms. Kay Jones

Consumer Oil Company, Inc.

P.O. Box 216

Bendena, KS 66008

coil@rainbowtel.net

785-988-4459 (office)

\subsubsection{KDHE Contacts for the Former CCC/USDA Facility}

\author{
KDHE Program Manager \\ Chris Carey, PG \\ Site Restoration Unit Chief \\ Remedial Section \\ Bureau of Environmental Remediation \\ Kansas Department of Health and Environment \\ Curtis State Office Building \\ 1000 SW Jackson Street, Suite 410 \\ Topeka, KS 66612-1367 \\ CCarey@kdheks.gov \\ 785-296-0225 (office) \\ 785-250-4750 (cell) \\ Section Chief of the Remedial Section \\ Rick L. Bean, LG \\ Chief, Remedial Section \\ Bureau of Environmental Remediation \\ Kansas Department of Health and Environment \\ Curtis State Office Building \\ 1000 SW Jackson Street, Suite 410 \\ Topeka, KS 66612-1367 \\ rbean@kdheks.gov \\ 785-296-1675 (office)
}

KDHE Project Manager

Holly Burke

Remedial Section/Site Restoration Unit Bureau of Environmental Remediation Kansas Department of Health and Environment Curtis State Office Building 1000 SW Jackson Street, Suite 410

Topeka, KS 66612-1367

hburke@kdheks.gov

785-296-6242 (office) 


\subsubsection{CCC/USDA Contacts}

Project Manager

Caroline H. Roe

Environmental Protection Specialist

Farm Service Agency

Conservation and Environmental Programs

Division

U.S. Department of Agriculture

STOP 0513

1400 Independence Avenue, SW

Washington, D.C. 20250-0513

Caroline.Roe@wdc.usda.gov

(202) 720-9964 (office)

(202) 720-4619 (office)
Legal Counsel

Gary M. Fremerman

Senior Counsel

Office of the General Counsel/Conservation and Environment Division

U.S. Department of Agriculture

Room 2340 South Building

1400 Independence Avenue, SW

Washington, DC 20250

gary.fremerman@ogc.usda.gov

202-720-8041 (office)

\subsubsection{Doniphan County Rural Water District No. 2 Contact}

Chairman

Gary Godfrey

Doniphan County Rural Water District No. 2

P.O. Box 136

Bendena, KS 66008

785-988-4482 (office)

\subsubsection{Argonne Contacts}

Lorraine M. LaFreniere, $\mathrm{PhD}$, Manager

Applied Geosciences and Environmental Management Section

Environmental Science Division

9700 South Cass Avenue

Argonne, IL 60439-4843

630-252-7969

lafreniere@anl.gov

Robert A. Sedivy

Bendena Technical Project Manager

Environmental Science Division

9700 South Cass Avenue

Argonne, IL 60439-4843

402-465-9021

rsedivy@anl.gov
James Hansen

Community Relations Representative

Environmental Science Division

Argonne National Laboratory

955 L'Enfant Plaza SW, Suite 6000

Washington, DC 20024

202-488-2453

hansenj@anl.gov 
TABLE 3.1 Emergency information for the investigation at Bendena, Kansas. ${ }^{a}$

\begin{tabular}{|c|c|c|}
\hline Resource & Telephone Number & Name \\
\hline All Emergencies & 911 & - \\
\hline Medical Care & $\begin{array}{l}855-479-4477 \\
\text { (toll free) }\end{array}$ & $\begin{array}{l}\text { Atchison Hospital } \\
800 \text { Ravenhill Drive } \\
\text { Atchison, Kansas }\end{array}$ \\
\hline Fire Protection (nonemergency) & $785-985-3711$ & $\begin{array}{l}\text { Doniphan County Sheriff } \\
219 \text { South Main Street } \\
\text { Troy, Kansas }\end{array}$ \\
\hline Police (nonemergency) & $785-985-3711$ & $\begin{array}{l}\text { Doniphan County Sheriff } \\
219 \text { South Main Street } \\
\text { Troy, Kansas }\end{array}$ \\
\hline Industrial Hygiene & $630-252-3310$ & Argonne-Industrial Hygiene \\
\hline Safety & $630-988-9706$ & $\begin{array}{l}\text { EVS Divisionc Field Safety Coordinator } \\
\text { (Monte Brandner) }\end{array}$ \\
\hline \multirow[t]{4}{*}{ Management } & $630-252-4878$ & $\begin{array}{l}\text { EVS Division }{ }^{C} \text { Environmental, Safety, and Health } \\
\text { Coordinator (Bill Gasper) }\end{array}$ \\
\hline & $630-252-7969$ & AGEM Program Manager (Lorraine LaFreniere) \\
\hline & $\begin{array}{l}630-252-1275 \\
630-408-7114\end{array}$ & AGEM Field Project Manager (David Surgnier) \\
\hline & $402-465-9021$ & AGEM Technical Project Manager (Robert Sedivy) \\
\hline Security & $\begin{array}{l}630-252-5737 \\
630-252-5731\end{array}$ & $\begin{array}{l}\text { Argonne-Operations Security (workdays) } \\
\text { (after hours and weekends) }\end{array}$ \\
\hline Poison Control & $\begin{array}{l}800-222-1222 \text { or } \\
913-588-6633\end{array}$ & $\begin{array}{l}\text { Mid-America Poison Control Center, } \\
\text { University of Kansas Medical Center }\end{array}$ \\
\hline Utilities Survey & $\begin{array}{l}800-344-7233 \\
800-D I G-S A F E\end{array}$ & Kansas One Call, Wichita, Kansas \\
\hline
\end{tabular}

a Post this table in the field operations base.

b The route from Bendena to the Atchison Hospital is shown in Figure 3.3.

c Environmental Science Division at Argonne. 


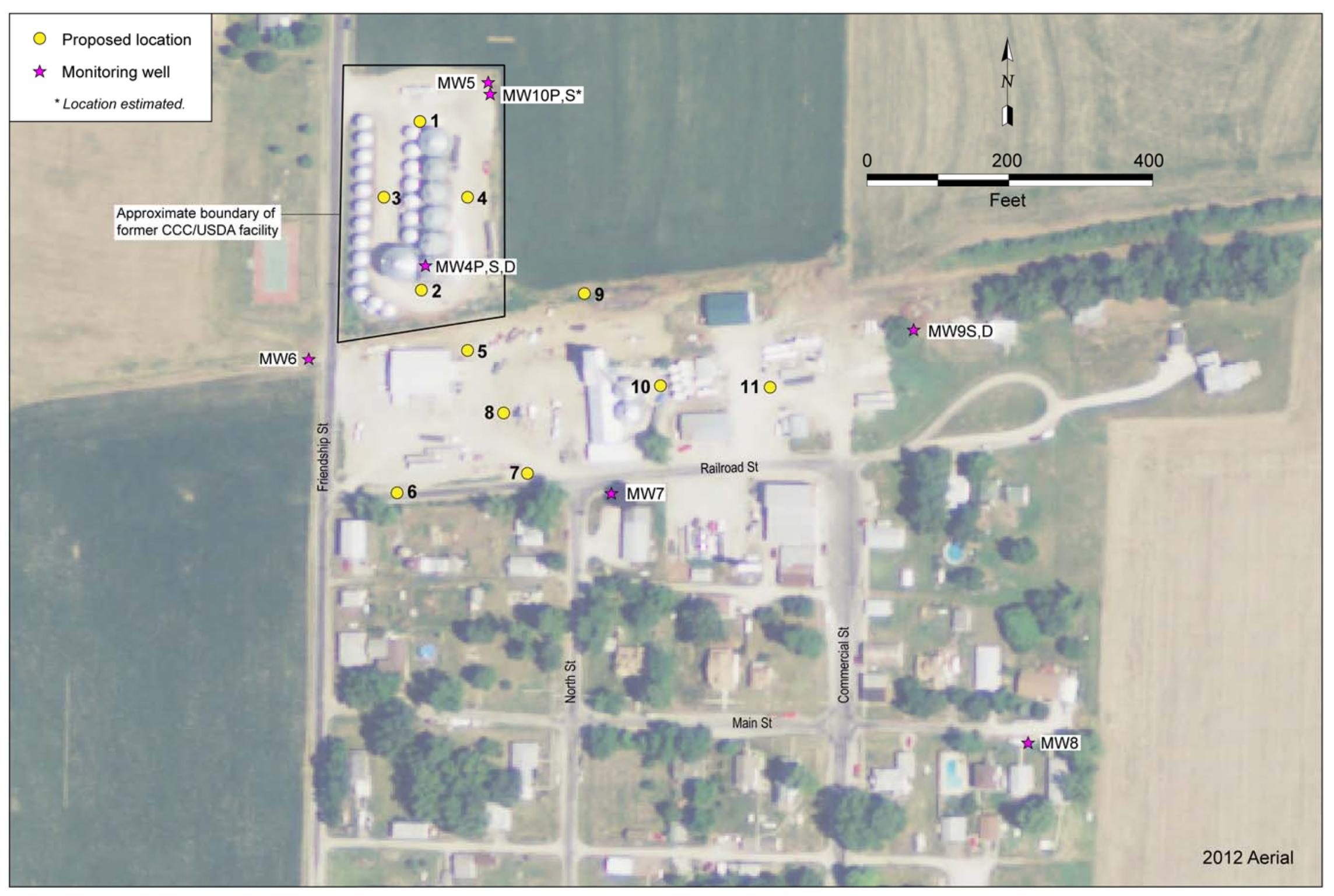

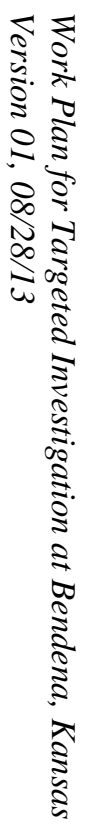

FIGURE 3.1 Proposed locations for investigative borings 1-11, with locations of existing monitoring wells. Source of photograph: NAIP (2012). 


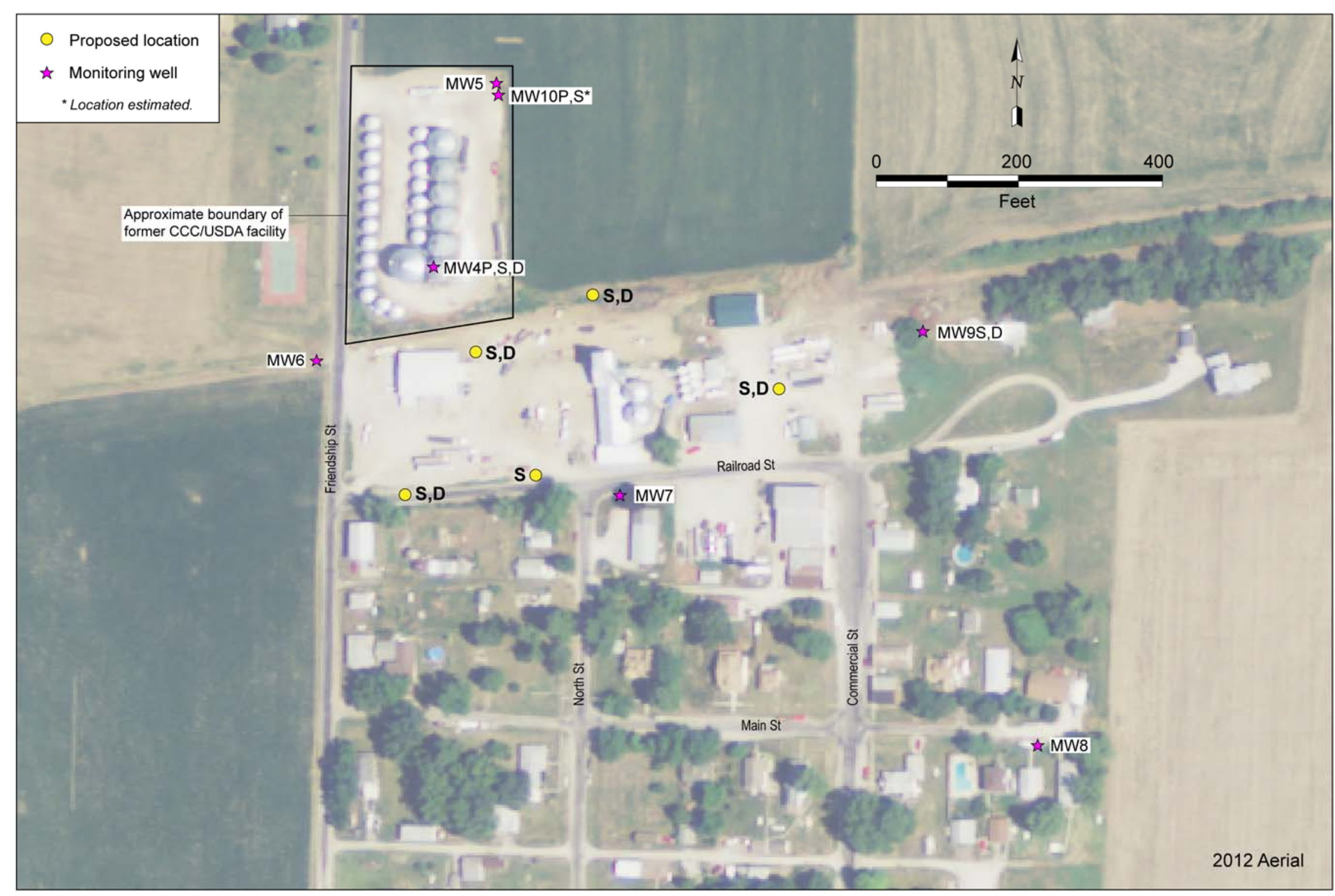

FIGURE 3.2 Proposed locations for installation of approximately nine new monitoring wells in the shallow and lower aquifers. Source of photograph: NAIP (2012). 


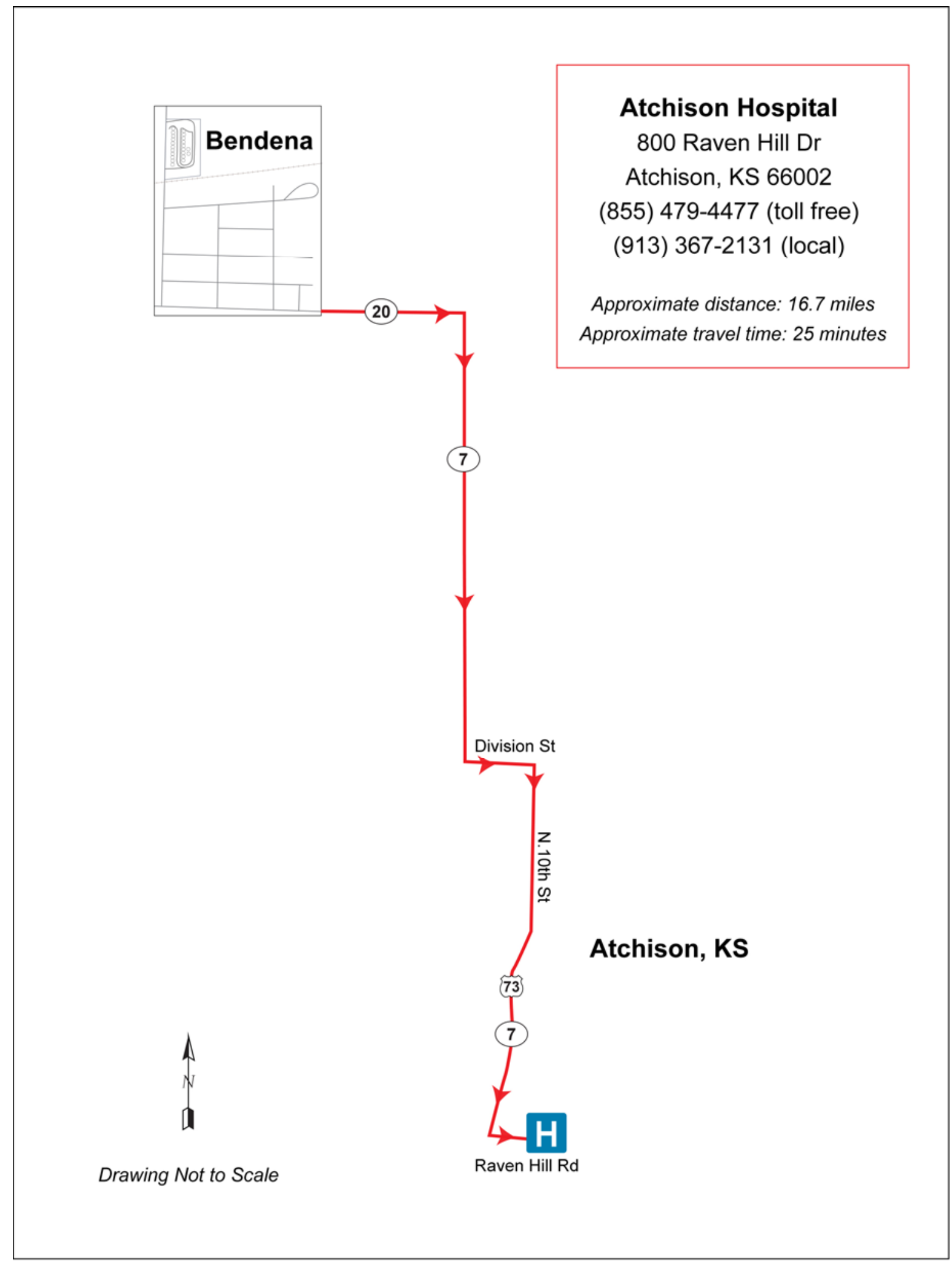

FIGURE 3.3 Emergency route from Bendena to the Atchison Hospital in Atchison, Kansas. 


\section{References}

Aber, J., 1988, “The Glaciation of Kansas” Heritage of the Great Plains 21(4):1-36.

Argonne, 2002, Final Master Work Plan: Environmental Investigations at Former CCC/USDA Facilities in Kansas, 2002 Revision, ANL/ER/TR-02/004, prepared for the Commodity Credit Corporation, U.S. Department of Agriculture, Washington, D.C., by Argonne National Laboratory, Argonne, Illinois, December.

Bayne, C., 1973, Geohydrology of Doniphan County, Northeastern Kansas, Hydrogeologic Investigations Atlas HA-462, U.S. Geological Survey, U.S. Government Printing Office, Washington, D.C.

Bayne, C., and W. Schoewe, 1967, Geology and Ground-Water Resources of Brown County, Kansas, Bulletin 229, Kansas Geological Survey, University of Kansas, Lawrence, Kansas.

CCC/USDA, 2013, data transmittal from C. Roe (Commodity Credit Corporation, U.S. Department of Agriculture, Washington, D.C.), to L.M. LaFreniere (Environmental Science Division, Argonne National Laboratory, Argonne, Illinois), summarizing the results of KDHE groundwater sampling for VOCs analyses at selected private wells in the Bendena area on February 13-14, 2013, May 23.

Dort, W., 1987, "Salient Aspects of the Terminal Zone of Continental Glaciation in Kansas," pp. 55-66 in Quaternary Environments of Kansas, Guidebook 5, Kansas Geological Survey, University of Kansas, Lawrence, Kansas.

Duncan, J.H., 1964, Engineers Report File - Bendena - Water, Doniphan County Rural Water District No. 2, memorandum from Duncan (Kansas State Department of Health, Topeka, Kansas), to the Kansas State Department of Health files, describing supplemental field notes pertaining to the 1964 final inspection of the new Doniphan County RWD 2 public water supply (PWS1, related piping, etc.), December 1.

Godfrey, G., 2013, telephone conversation between Godfrey (Chairman, Doniphan County Rural Water District No. 2, Bendena, Kansas) and J. Hansen (Environmental Sciences Division, 
Argonne National Laboratory, Argonne, Illinois), concerning past and current sources of municipal water used by the town of Bendena, July 29.

GSI, 2011, Supplemental Investigation Work Plan Voluntary Cleanup Investigation and Interim Removal Revision 1.0 Bendena Ag East and West Facilities, prepared by Geotechnical Services, Inc., Kansas City, Missouri, for Bendena Ag, Inc., Bendena, Kansas, November 4.

GSI, 2012, Supplemental Investigation Work Plan Addendum Response to KDHE Comments Supplemental Voluntary Cleanup Investigation Bendena Ag East and West Properties, Bendena, Kansas, prepared by Geotechnical Services, Inc., Kansas City, Missouri, for Bendena Ag, Inc., Bendena, Kansas, February 22.

KDHE, 1985a, letter from Gyula F. Kovach (Manager, Bureau of Water Protection, Kansas Department of Health and Environment, Topeka, Kansas) to Gary Godfrey (Chairman, Board of Directors, Doniphan County Rural Water District No. 2, Bendena, Kansas), transmitting results of VOCs analyses on samples collected from the district's well No. 1 on February 26, March 11, and March 22, with recommendation for notification of results to district customers, April 9.

KDHE, 1985b, letter from Gyula F. Kovach (Manager, Bureau of Water Protection, Kansas Department of Health and Environment, Topeka, Kansas) to Gary Godfrey (Chairman, Board of Directors, Doniphan County Rural Water District No. 2, Bendena, Kansas), transmitting a directive from the KDHE to RWD 2 to obtain a contaminant-free water source for the district within 180 days, September 5.

KDHE, 1986a, letter from Gyula F. Kovach (Manager, Bureau of Water Protection, Kansas Department of Health and Environment, Topeka, Kansas) to Gary Godfrey (Chairman, Board of Directors, Doniphan County Rural Water District No. 2, Bendena, Kansas), requesting a plan and schedule to provide contaminant-free water to district customers, February 20.

KDHE, 1986b, letter from Gyula F. Kovach (Manager, Bureau of Water Protection, Kansas Department of Health and Environment, Topeka, Kansas) to Gary Godfrey (Chairman, Board of Directors, Doniphan County Rural Water District No. 2, Bendena, Kansas), extending the deadline for supplying contaminant-free water to district customers until November 1, 1986, March 13. 
KDHE, 1987, Preliminary Assessment of the Doniphan County Rural Water District No. 2, Bendena, Kansas (EPA ID\# KSD981710304), prepared by G.P. Belt (Environmental Technician, Bureau of Environmental Remediation, Kansas Department of Health and Environment, Topeka, Kansas), December 15.

KDHE, 1988, Final Site Inspection Report for the Doniphan County Rural Water District \#2 (EPA/KDHE I.D. \#KSD981710304), prepared for U.S. Environmental Protection Agency, Region VII, Kansas City, Kansas, by Technical Services Section, Bureau of Environmental Remediation, Kansas Department of Health and Environment, Topeka, Kansas, September 15.

KDHE, 1998, Public Water Supply Site Comprehensive Investigation, Bendena, Kansas, prepared for the Bureau of Environmental Remediation, Kansas Department of Health and Environment, Topeka, Kansas, by BE\&K/Terranext, Lenexa, Kansas, May 21.

KDHE, 2005, Scope of Work for Site Monitoring, Policy BER-RS-036, Remedial Section, Bureau of Environmental Remediation, Kansas Department of Health and Environment, Topeka, Kansas, December (http://www.kdheks.gov/ber/policies/BER_RS_036_SOW.pdf).

KDHE, 2010a, Bendena RWD \#2, PWS \#1 Site, Bendena, Kansas, Supplemental Site Investigation Report, prepared by T. Wells, Geology Associate, Bureau of Environmental Remediation, Remedial Section/State Response \&and Property Redevelopment Unit, Kansas Department of Health and Environment, Topeka, Kansas, May.

KDHE, 2010b, Risk Based Standards for Kansas: RSK Manual - 5th Version, Bureau of Environmental Remediation, Kansas Department of Health and Environment, Topeka, Kansas, June (www.kdheks.gov/remedial/rsk_manual_page.htm).

KDHE, 2012, Private Well Sampling Work Plan for Former CCC/USDA Grain Storage Sites, memorandum from P. Chaffee to C. Carey (Site Restoration Unit, Remedial Section, Bureau of Environmental Remediation, Kansas Department of Health and Environment, Topeka, Kansas), December 3.

Larsen, 2012a, Results of the Comprehensive Nitrate and Ammonia Investigation Conducted at the Johnson Farms, Inc., 924 Friendship Road, Bendena, KS, prepared for Johnson Farms, Inc., Bendena, Kansas, by Larsen and Associates, Inc., Lawrence, Kansas, February 8. 
Larsen, 2012b, Results of the Phase II Comprehensive Nitrate and Ammonia Investigation Conducted at the Johnson Farms, Inc., 924 Friendship Road, Bendena, KS, prepared for Johnson Farms, Inc., Bendena, Kansas, by Larsen and Associates, Inc., Lawrence, Kansas, July 17.

Novak and Lay, 1987, letter from D.J. Novak (Novak and Lay Engineers/Surveyors, Hiawatha, Kansas) to Karl Mueldener (Kansas Department of Health and Environment, Topeka, Kansas), describing alternatives for providing contaminant-free water to Doniphan County Rural Water District No. 2 customers, including description of selected alternative and preliminary engineer's cost estimate, March 25.

SDOH, 1964, letter from J. Burris (Kansas State Department of Health, Environmental Health Services, Topeka, Kansas) to G. Clark (Chairman, Rural Water District No. 2, Bendena, Kansas) with recommendations regarding operations and approving the RWD 2 water supply, November 17.

USDA, 1966, Photo YZ-3GG-66, U.S. Department of Agriculture Photographic Laboratory, Salt Lake City, Utah, September 9.

USGS, 1969, Bendena Quadrangle, Doniphan County, Kansas, U.S. Geological Survey 7.5-Minute Topographic Map Series, U.S. Government Printing Office, Washington, D.C.

USGS, 1991, Digital Orthophoto Quadrangle, DI000034765, U.S. Geological Survey, EROS Data Center, Sioux Falls, South Dakota, on CD-ROM, October 16. 


\section{Appendix A:}

\section{Monitoring Well Registrations}




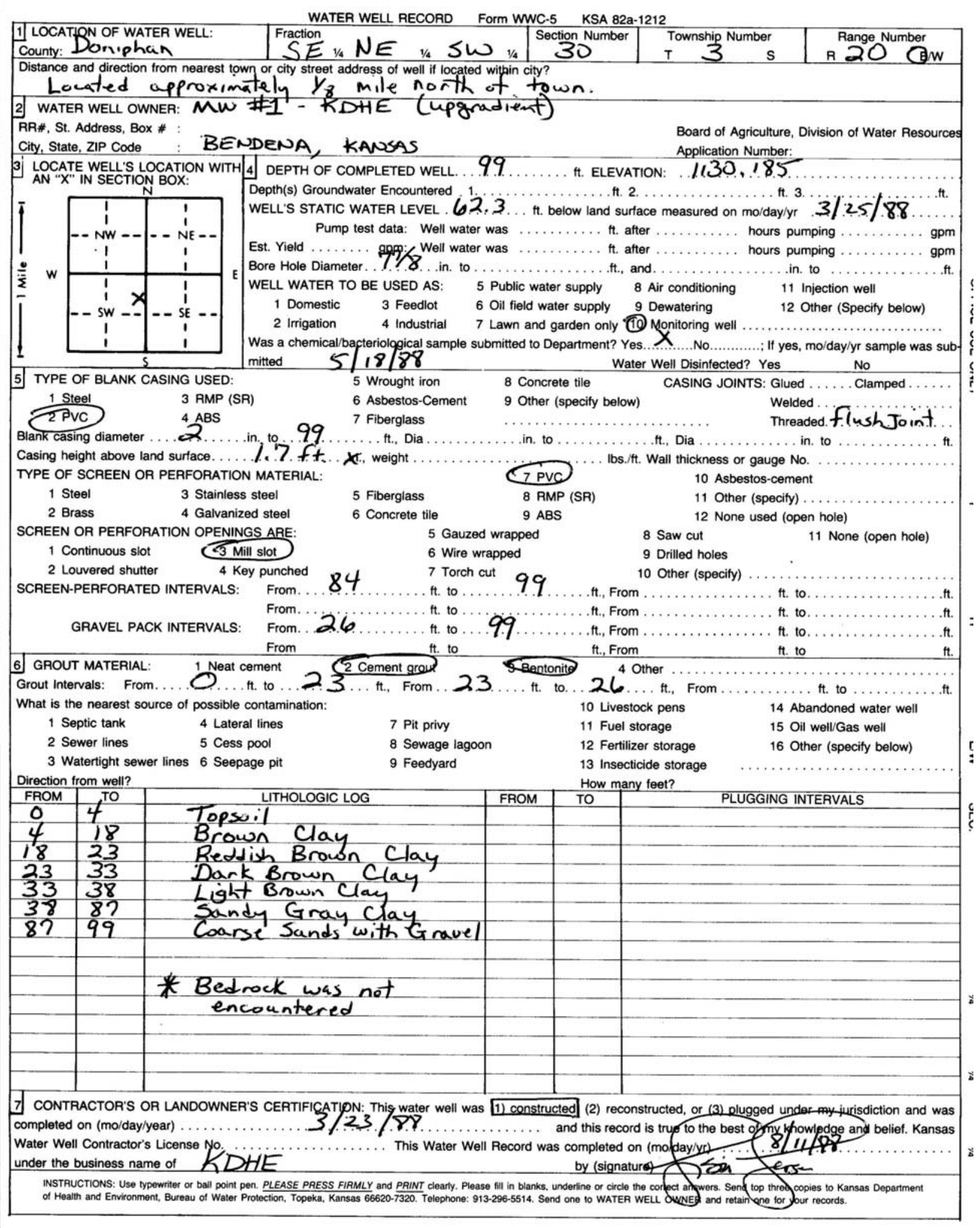


WATER WELL PLUGGING RECORD Form WWC.5P KSA82a-1212 IDNO.

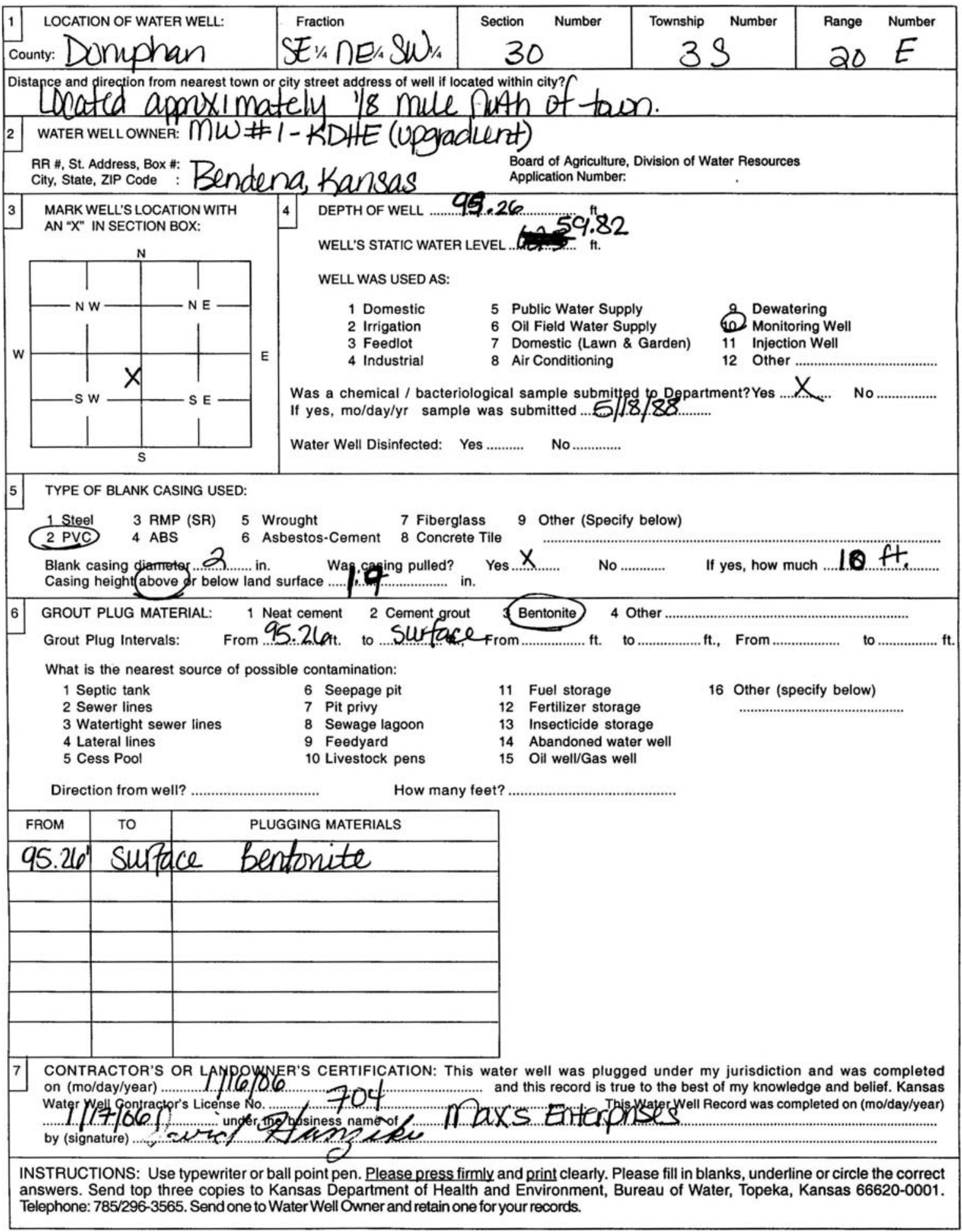




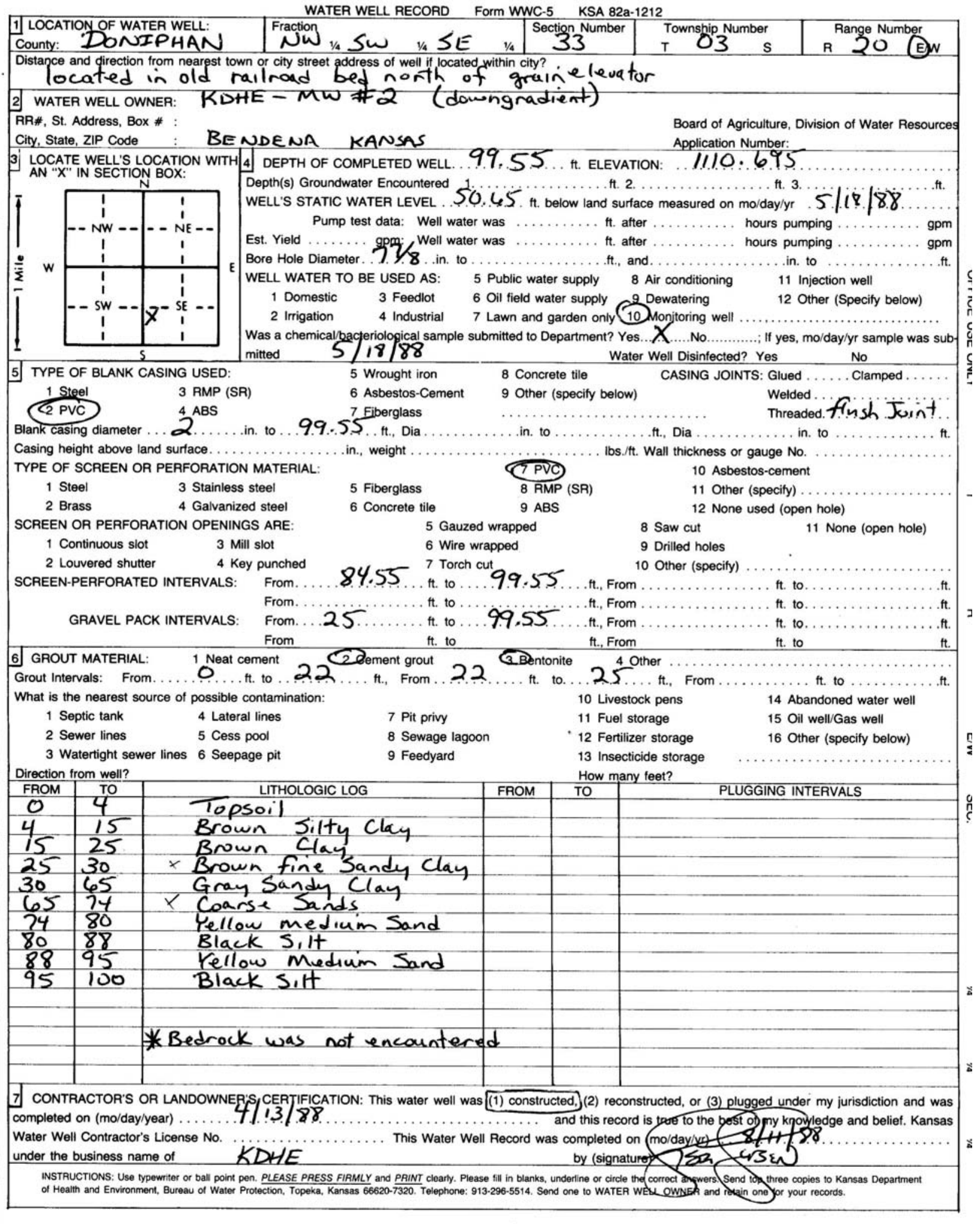


WATER WELL PLUGGING RECORD FOrm WWC.5P KSA82a-1212 IDNO.

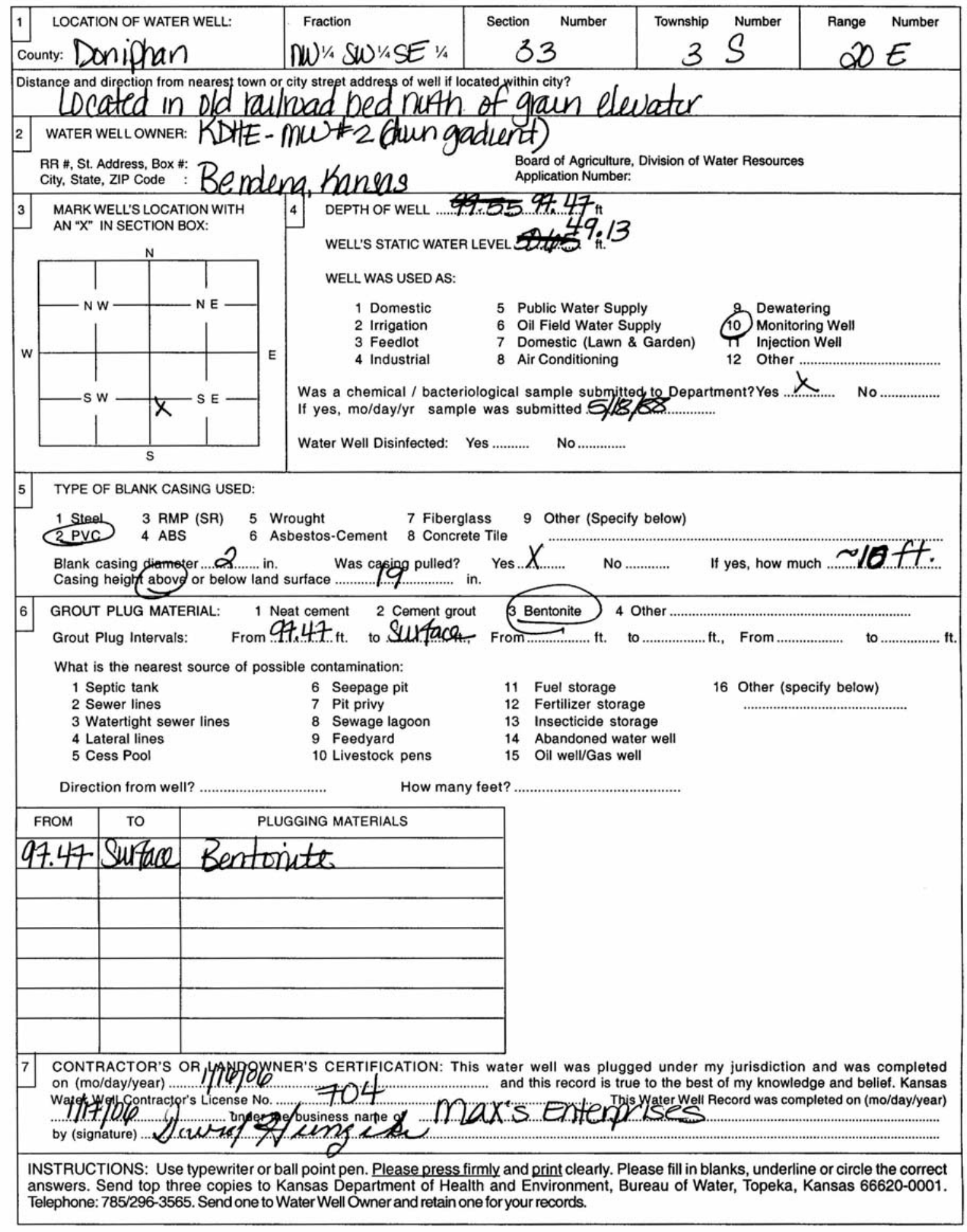




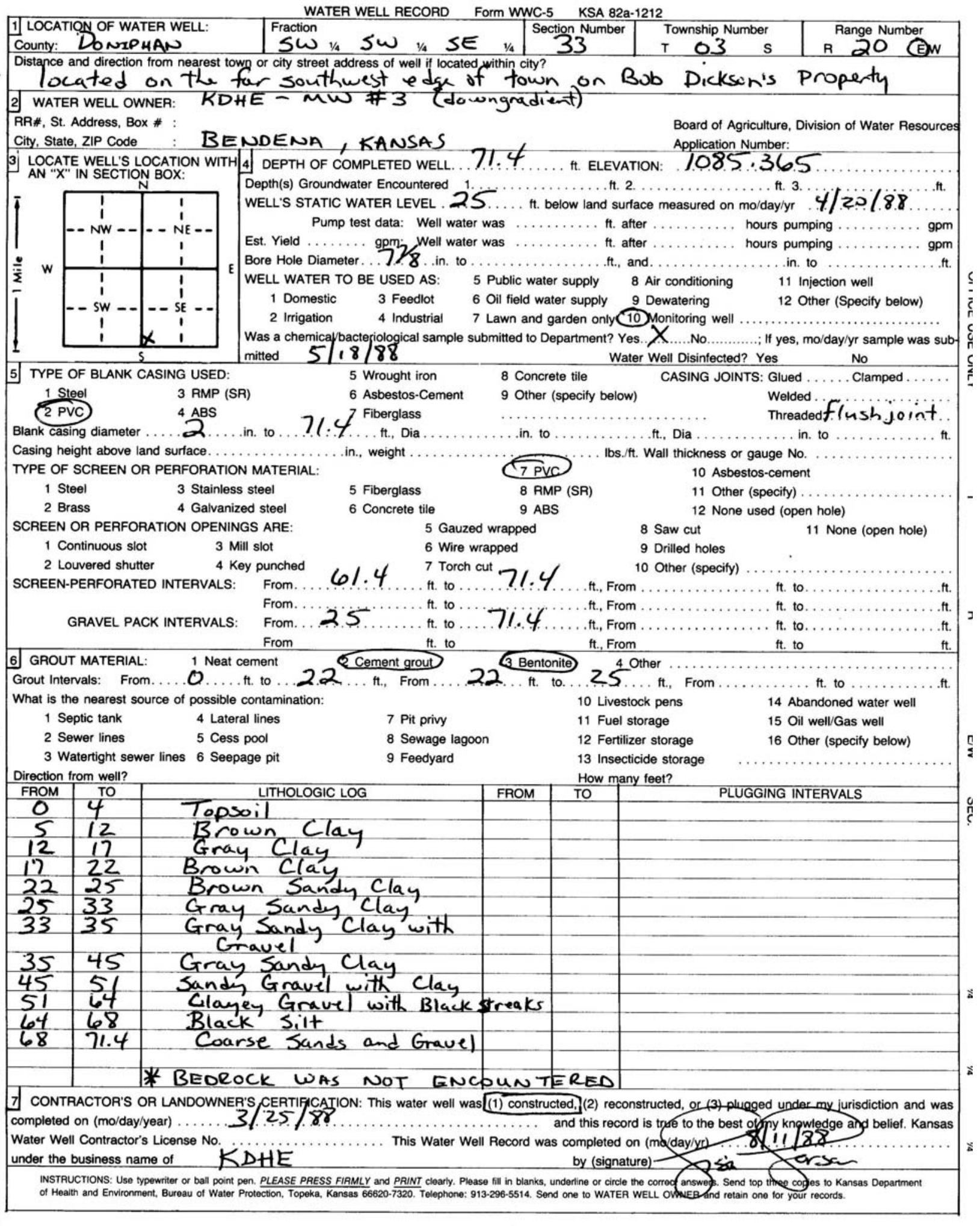


WATER WELL PLUGGING RECORD FOrm WWC-5P KSA82a-1212 IDNO. 2456

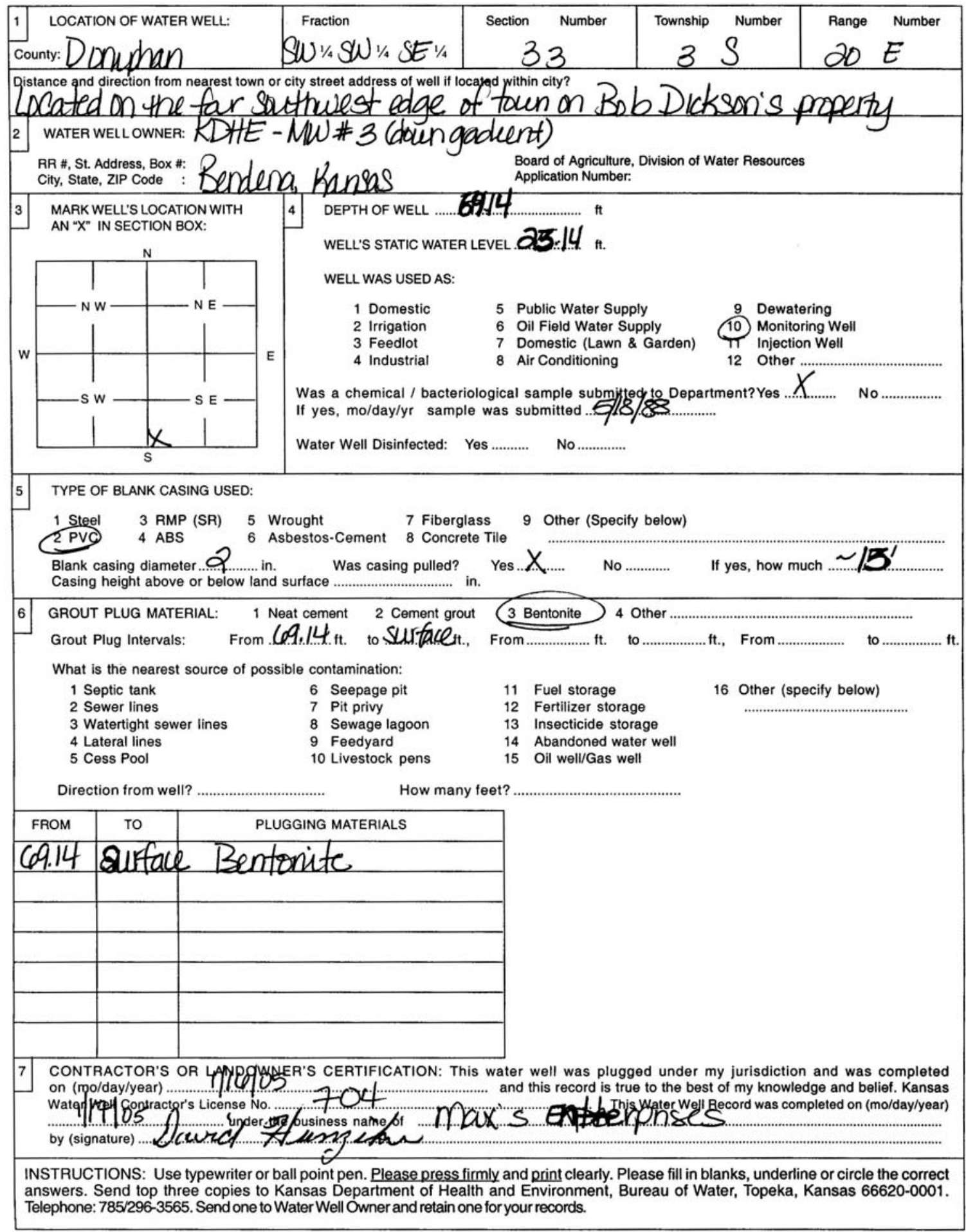


WATER WELL RECORD Form WWC-5 KSA 82a-1212

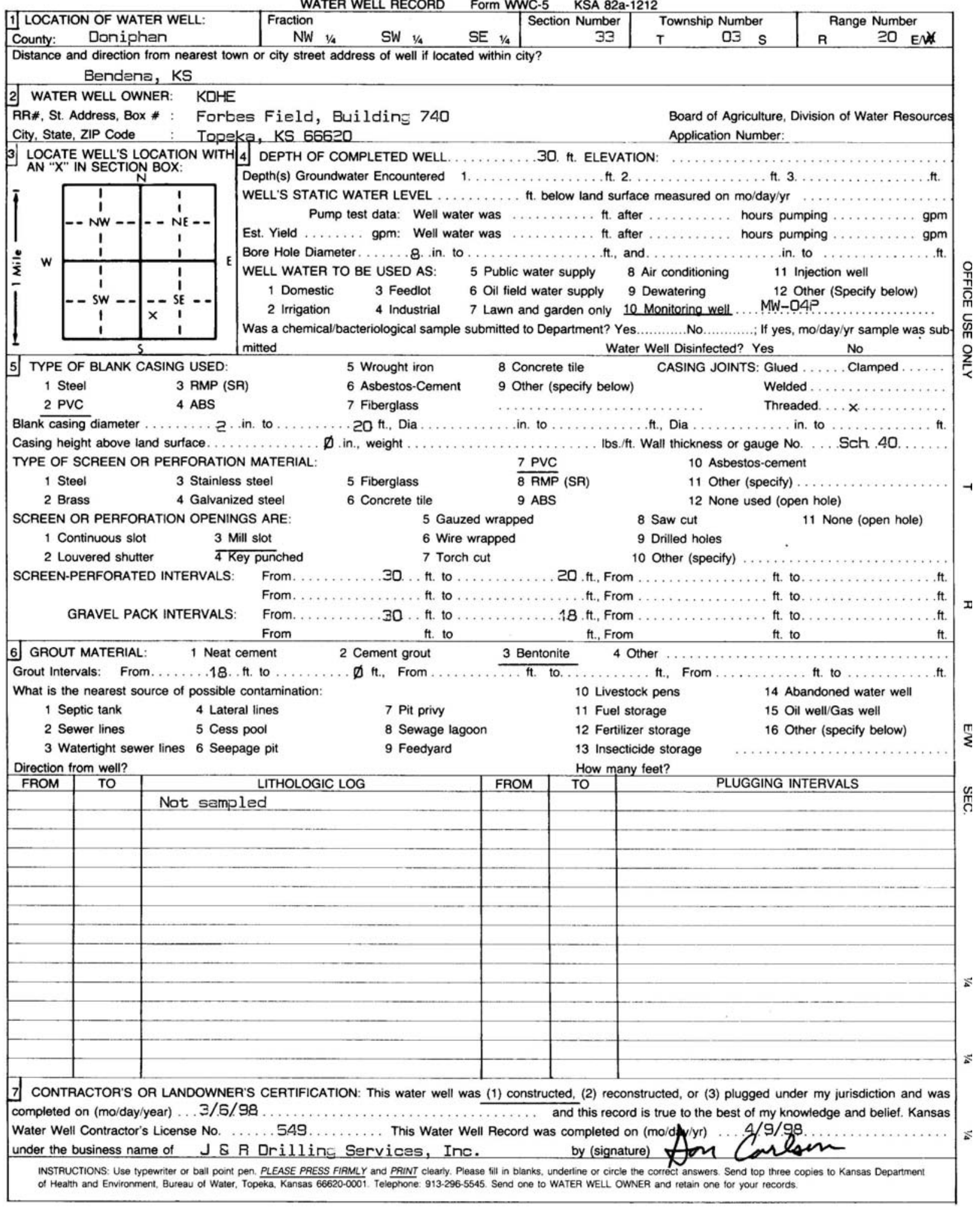




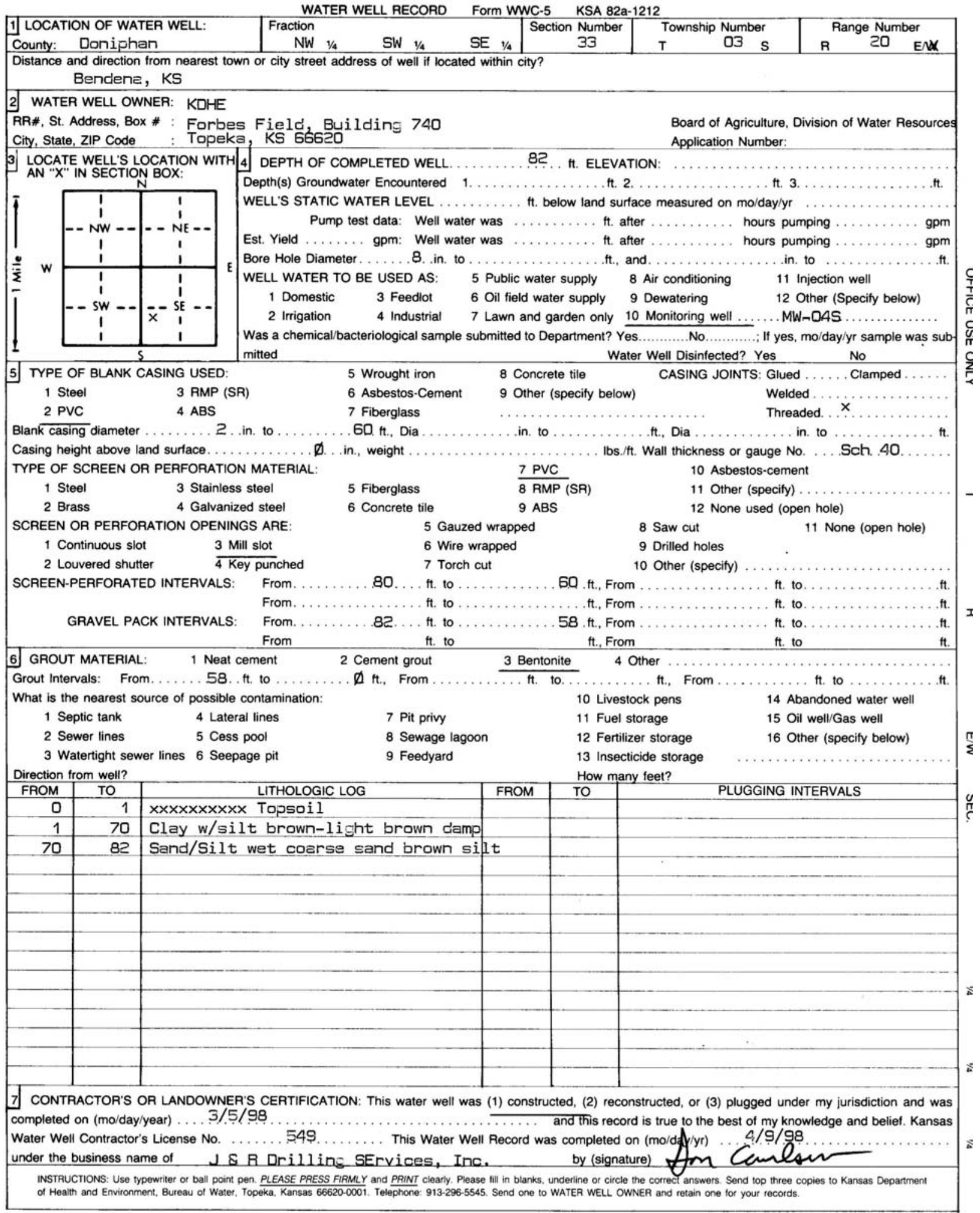


WATER WELL RECORD Form WWC-5 KSA 82a-1212

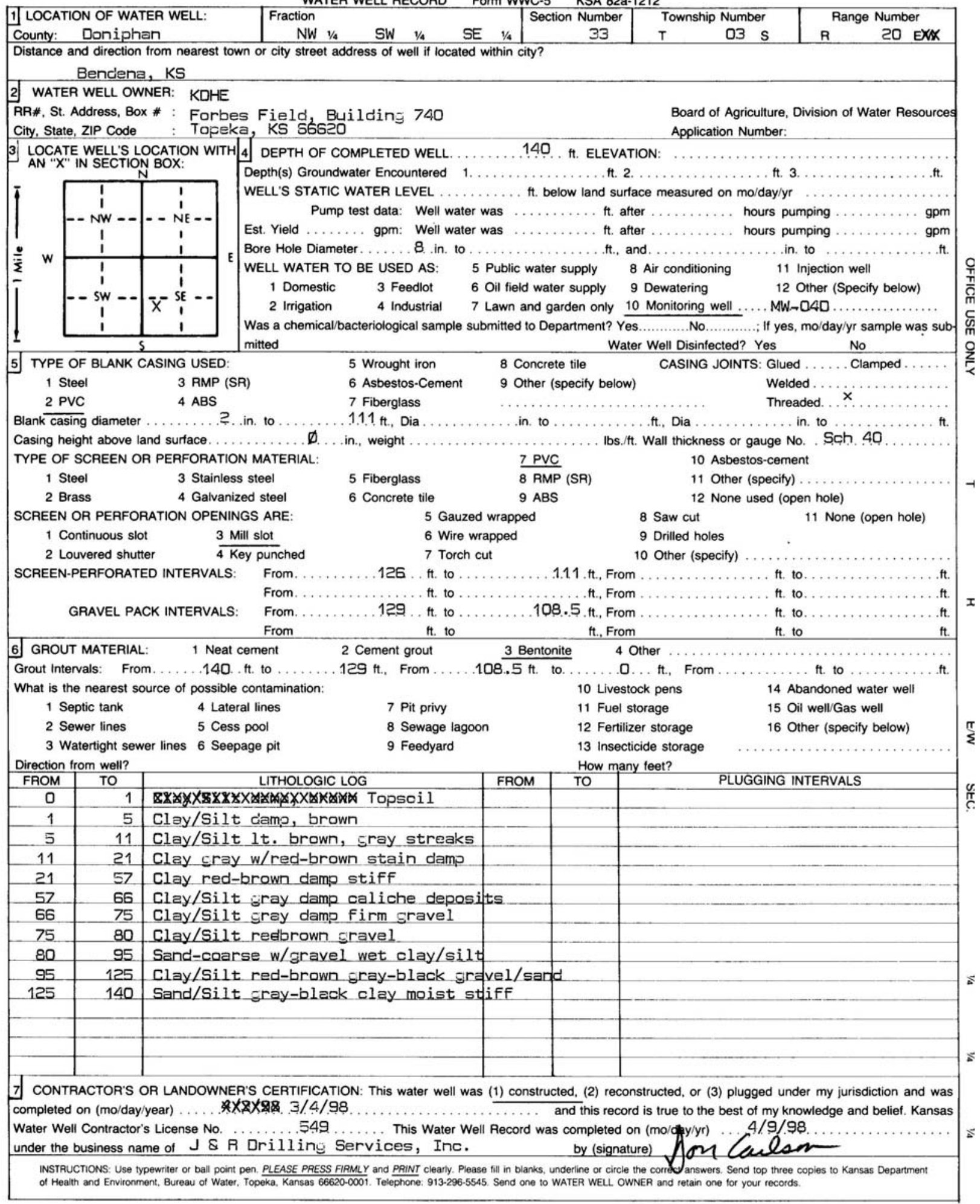




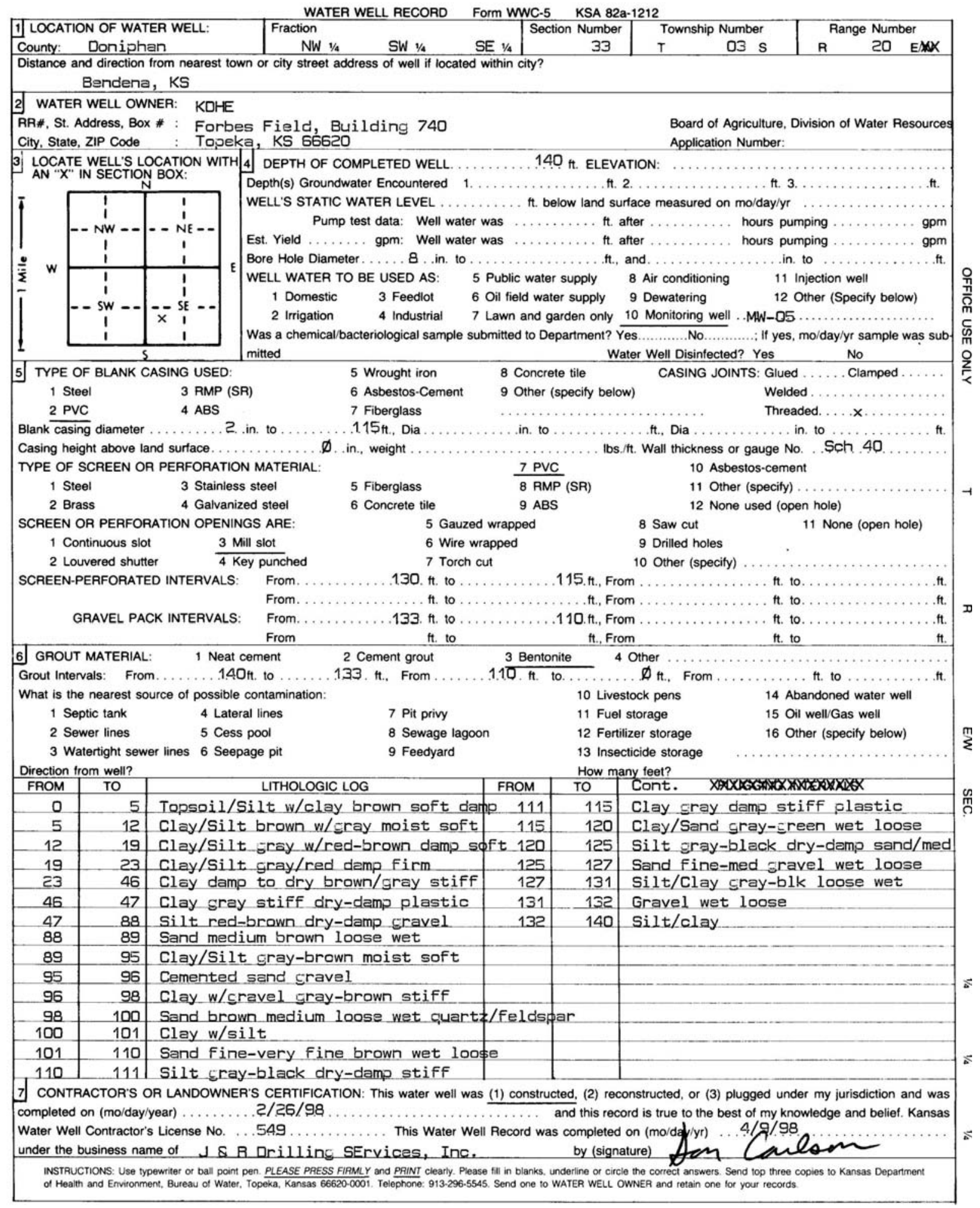




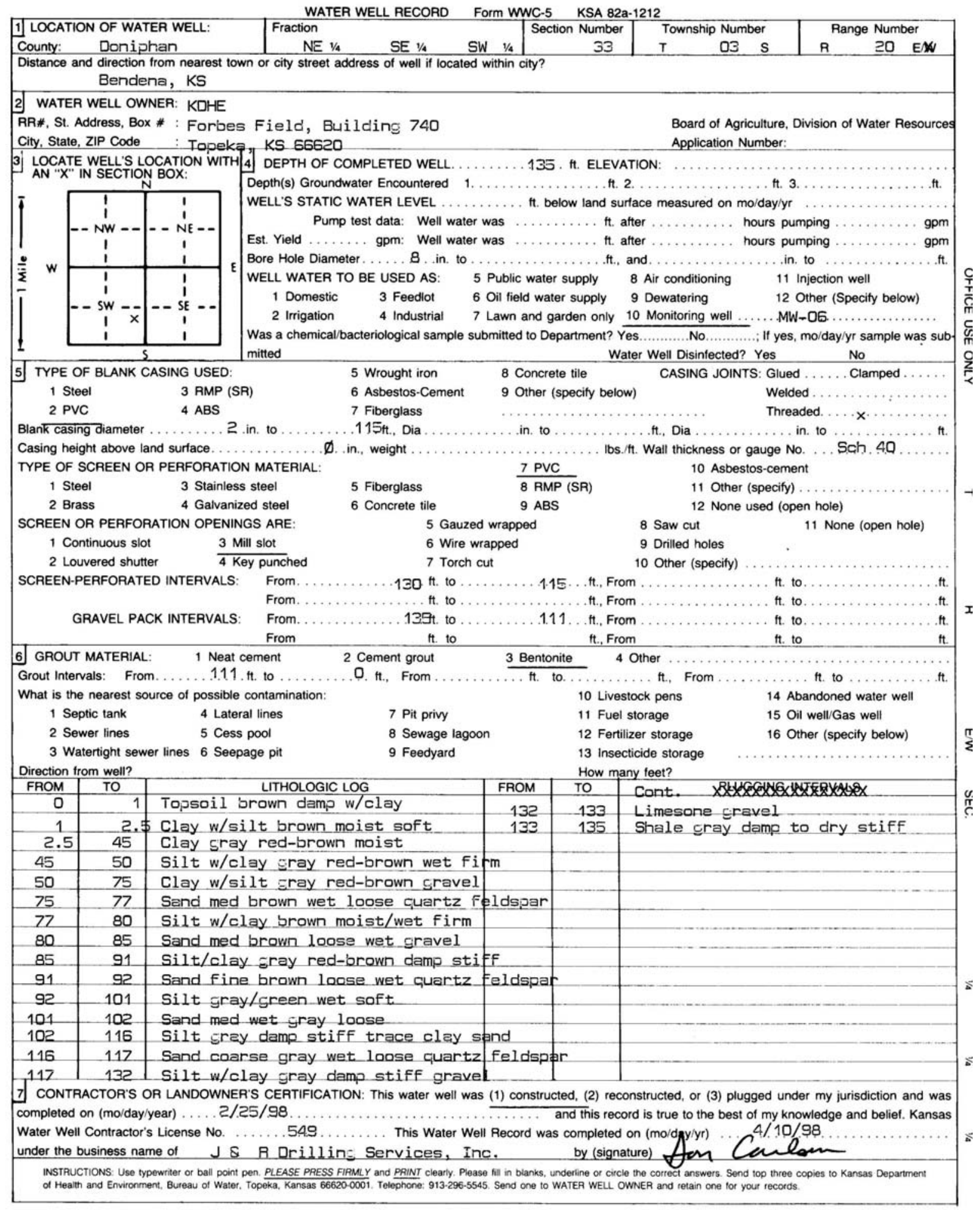


WATER WELL RECORD Form WWC-5 KSA 82a-1212

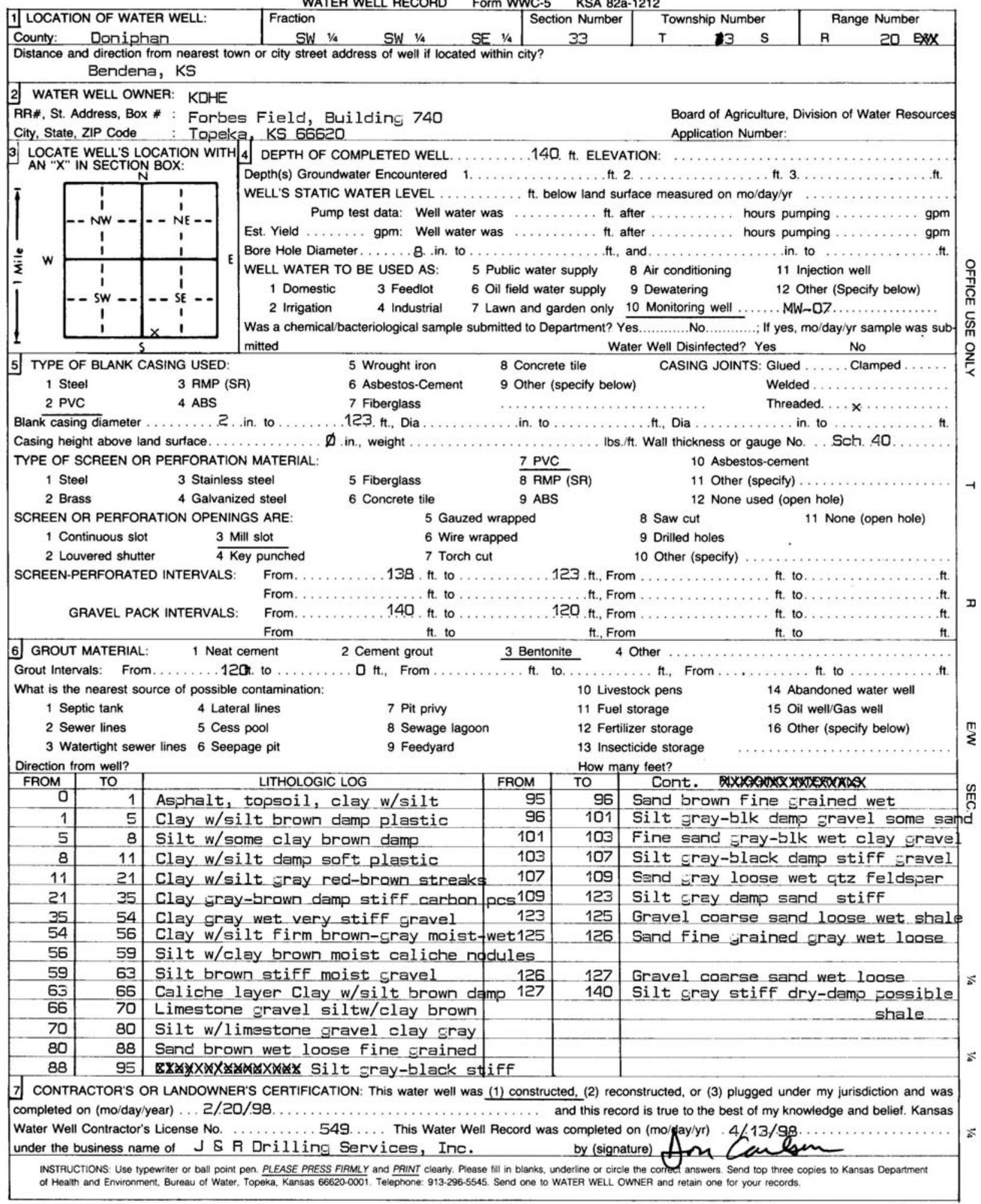




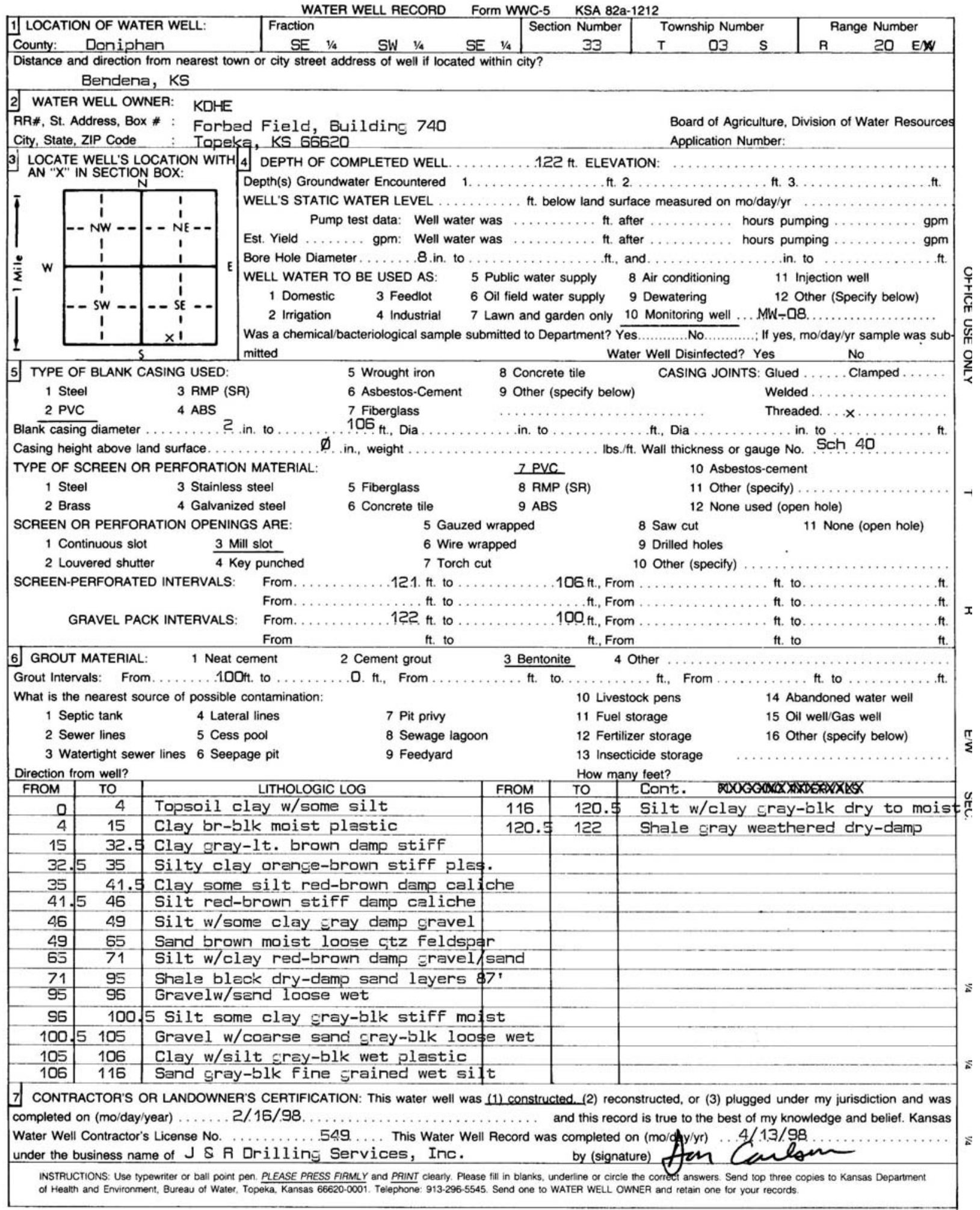




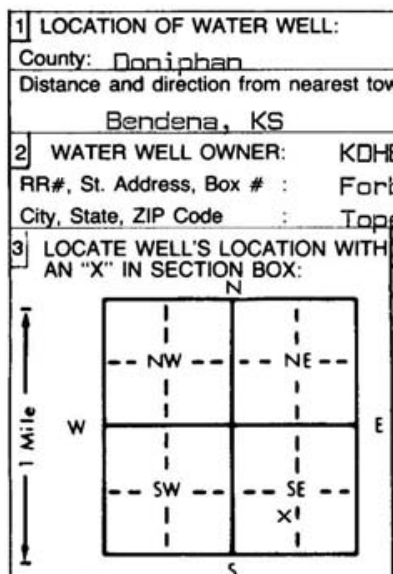

5 TYPE OF BLANK CASING USED 1 Steel 3 RMP (SR) 2 PVC

4 ABS

Blank casing diameter .......... . in. to

Casing height above land surface. .

TYPE OF SCREEN OR PERFORATION MATERIAL

1 Steel

2 Brass

3 Stainless steel

4 Galvanized steel

SCREEN OR PERFORATION OPENINGS ARE:

1 Continuous slot

2 Louvered shutter

3 Mill slot

4 Key punched Fraction mitted

WATER WELL RECORD Form WWC-5 KSA 82a-1212

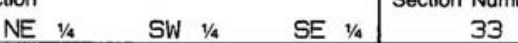

\begin{tabular}{cc|cc}
\multicolumn{2}{c|}{ Township Number } & \multicolumn{2}{|c}{ Range Number } \\
T & 03 S & R & 20
\end{tabular}

city street address of well if located within city?

T

03

21

Forbes Field, Building 740

Board of Agriculture, Division of Water Resources Application Number:

4 DEPTH OF COMPLETED WELL. .........65. ft. ELEVATION

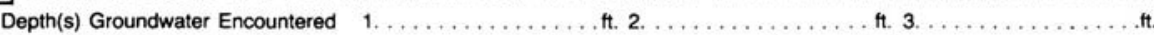

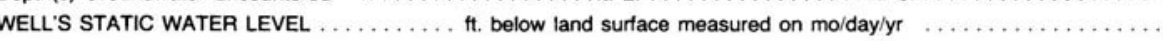

Pump test data: Well water was $\ldots \ldots \ldots \ldots$ th. after $\ldots \ldots \ldots$ hours pumping ......... gpm

Est. Yield $\ldots \ldots \ldots$ gpm: Well water was $\ldots \ldots \ldots \ldots$ tt. after $\ldots \ldots \ldots$ hours pumping $\ldots \ldots \ldots$ gpm

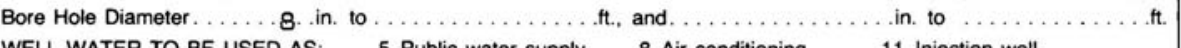

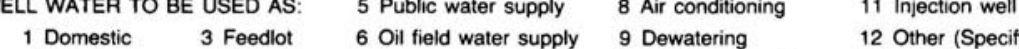

2 Irrigation $\quad 4$ Industrial 7 Lawn and garden only 10 Monitoring well .......MW-- 095.

Was a chemical/bacteriological sample submitted to Department? Yes............No............; if yes, mo/day/yr sample was sub-

Water Well Disinfected? Yes

\begin{tabular}{|c|c|c|}
\hline 5 Wrought iron & 8 Concrete tile & CASING JOINTS: Glued \\
\hline 6 Asbestos-Cement & 9 Other (specify below) & Welded \\
\hline 75 Fiberglass & .. & Threaded...x \\
\hline
\end{tabular}

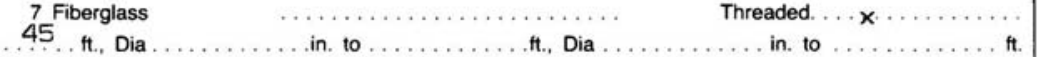

$₫$.in., weight ................. Ibs./ft. Wall thickness or gauge No. Sch. $40 \ldots \ldots \ldots$

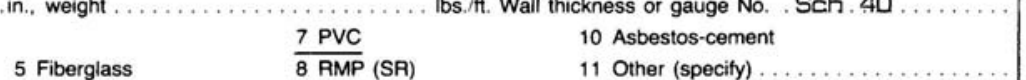

6 Concrete tile 9 ABS

12 None used (open hole)

5 Gauzed wrapped

8 Saw cut

11 None (open hole)

6 Wire wrapped

7 Torch cut

9 Drilled holes

10 Other (specify) $\ldots \ldots \ldots \ldots \ldots \ldots \ldots \ldots \ldots \ldots \ldots \ldots \ldots \ldots \ldots \ldots \ldots$

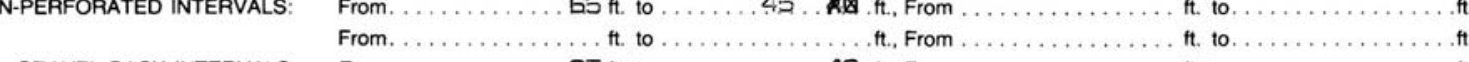

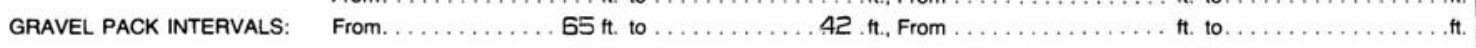

From th. to

it. to

th., From

$\frac{3 \text { Bentonite }}{\text {.... th. to. }}$

6 GROUT MATERIAL: 1 Neat cement

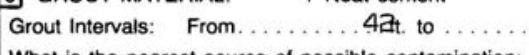

What is the nearest source of possible contamination:

1 Septic tank 4 Lateral lines

2 Sewer lines $\quad 5$ Cess pool

2 Cement grout
0. ft., From

4 Other

ft. From $\ldots \ldots \ldots \ldots \ldots \ldots \ldots \ldots \ldots \ldots$ tt. to

3 Watertight sewer lines 6 Seepage pit

7 Pit privy

8 Sewage lagoon

9 Feedyard
10 Livestock pens

11 Fuel storage

12 Fertilizer storage

13 Insecticide storage

How many feet?

Direction from well?

\begin{tabular}{|r|c|c|c|c|c|}
\hline FROM & TO & \multicolumn{1}{|c|}{ LITHOLOGIC LOG } & FROM & TO & PLU feet? \\
\hline 0 & 25 & Clay w/minor silt soft sray damp & & & \\
\hline 25 & 65 & Clay continued becomina firm & & & \\
\hline
\end{tabular}

14 Abandoned water well

15 Oil well/Gas well
16 Other (specify below)

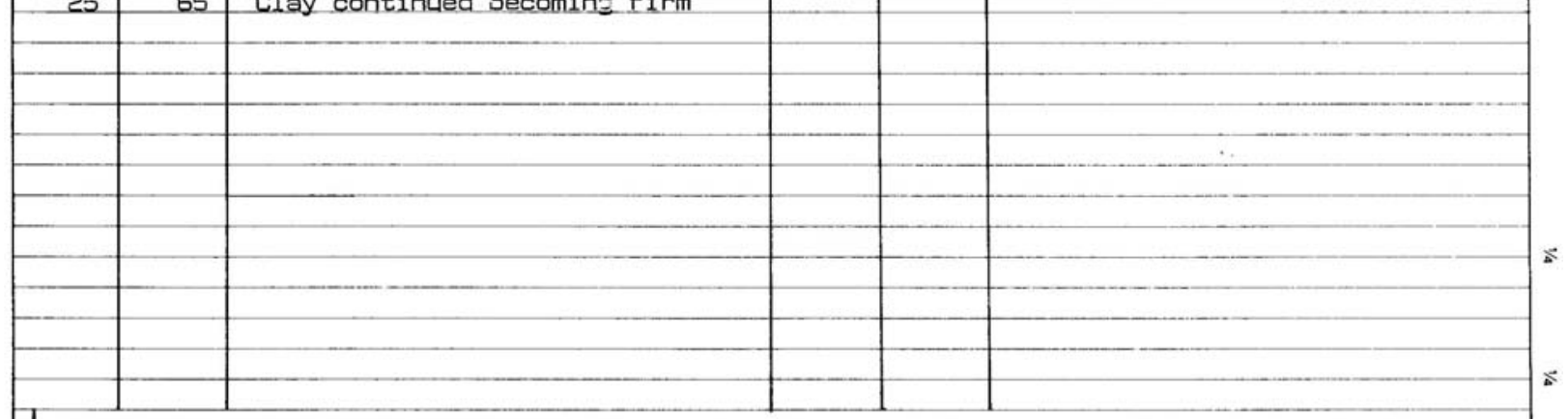

7 CONTRACTOR'S OR LANDOWNER'S CERTIFICATION: This water well was (1) constructed, (2) reconstructed, or (3) plugged under my jurisdiction and was completed on (mo/day/year) . . . $3 / 10 / 98 \ldots \ldots \ldots \ldots \ldots \ldots \ldots \ldots \ldots \ldots \ldots \ldots \ldots$ and this record is true to the best of my knowledge and belief. Kansas

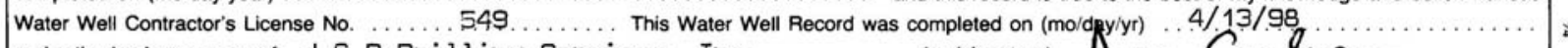
under the business name of $J \&$ R Drilling Services, Inc.

by (signature) INSTRUCTIONS: Use typewriter or ball point pen. PLEASE PRESS FIRMLY and PRINT clearly. Please fill in blanks, underline or circle the correch answers. Send top three copp
of Health and Environment, Bureau of Water, Topeka, Kansas 66620-0001. Telephone: 913-296-5545. Send one to WATER WELL OWNER and retain one for your records. 
WATER WELL RECORD Form WWC-5 KSA 82a-1212

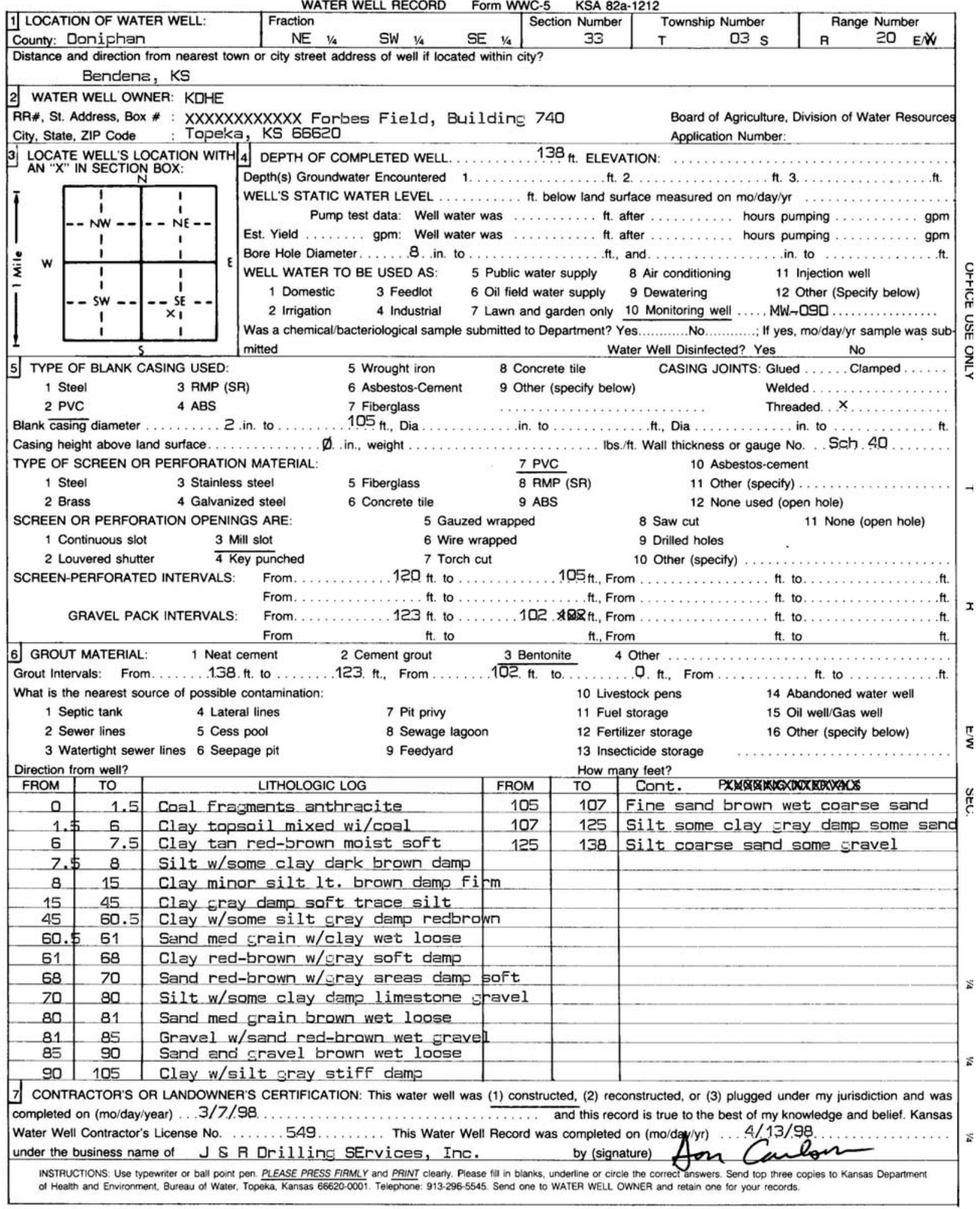


WATER WELL RECORD \begin{tabular}{|ll|}
\hline 1 LOCATION OF W \\
County: \\
\hline $\begin{array}{l}\text { Distance and direction fon } \\
\text { located within city? } 924\end{array}$ \\
\hline 2 WATER WELL O \\
RR\#, St. Address, B \\
City, State, ZIP Code
\end{tabular}

Form WWC-5

Division of Water Resources; App. No.

Section Number Township Number Range Number

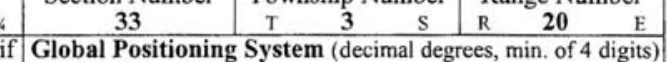

Latitude: NA

Longitude: NA

Elevation: NA

Datum: NA

Data Collection Method: legal survey

924 Friendship Road

MW10P

ft. 2 ft.

\section{DEPTH OF COMPLETED WELL 25.10}

Depth(s) Groundwater Encountered 1

WELL'S STATIC WATER LEVEL ........ fry. below land surface measured on mo/day/yr ...7/17/12..

ft. 3

$\mathrm{ft}$.

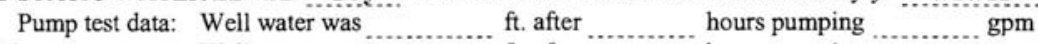
Est. Yield gpm: Well water was $\quad \mathrm{ft}$. after $\quad$ hours pumping gpm WELL WATER TO BE USED AS: 5 Public water supply 8 Air conditioning 11 Injection well 1 Domestic 3 Feed lot 6 Oil field water supply 9 Dewatering 12 Other (Specify below) 2 Irrigation 4 Industrial 7 Domestic (lawn \& garden) (10)Monitoring well

Was a chemical/bacteriological sample submitted to Department? Yes ..... No _. X ; If yes, mo/day/yrs Sample was submitted Water Well Disinfected? Yes No $\mathbf{X}$.

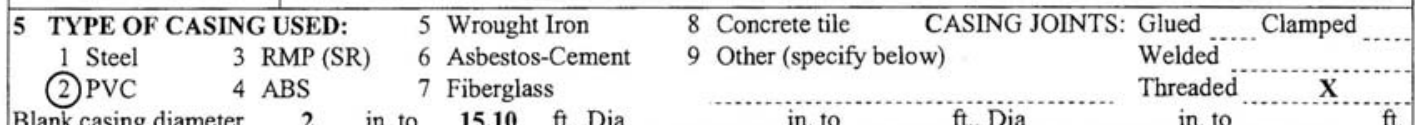
Blank casing diameter $2 \ldots$ in. to 15.10 ft., Dia

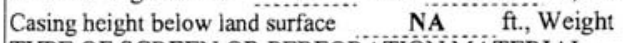
in to $\mathrm{ft}$ in to $\mathrm{lbs} . / \mathrm{ft}$. Wall thickness or gauge No.

TYPE OF SCREEN OR PERFORATION MATERIAI:

1 Steel 3 Stainless steel 5 Fiberglass (7) PVC 9 ABS 11 Other (specify)

2 Brass 4 Galvanized steel 6 Concrete tile 8 RM (SR) 10 Asbestos-Cement 12 None used (open hole) SCREEN OR PERFORATION OPENINGS ARE:

1 Continuous slot (3) Mill slot 5 Gauze wrapped 7 Torch cut 9 Drilled holes 11 None (open hole)

2 Louvered shutter 4 Key punched 6 Wire wrapped 8 Saw Cut 10 Other (specify)

SCREEN-PERFORATED INTERVALS: From $15.10 \quad \mathrm{ft}$. to $25.10 \ldots \mathrm{ft}$. From

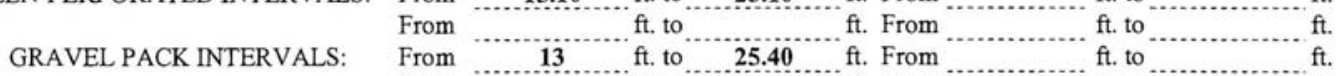

From ft. to ft. From $\quad$ ft.

6 GROUT MATERIAL: 1 Neat cement 2 Cement grout (3) Bentonite (4) Other Concrete: 0-1ft

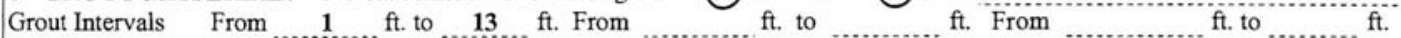
What is the nearest source of possible contamination:
1 Septic tank
4 Lateral lines 7 Pit privy 10 Livestock pens 13 Insecticide Storage
2 Sewer lines
5 Cess pool 8 Sewage lagoon (11) Fuel storage 14 Abandoned water well
16 Other (specify below)

3 Watertight sewer lines 6 Seepage pit 9 Feedyard 12 Fertilizer storage 15 Oil well/gas well

Direction from well? W How many feet? $\mathbf{5 0 f t}$

\begin{tabular}{|l|c|l|l|l|l|}
\hline FROM & TO & \multicolumn{1}{|c|}{ LITHOLOGIC LOG } & FROM & TO & PLUGGING INTERVALS \\
\hline $\mathbf{0}$ & $\mathbf{5}$ & Brown soft silty clay & & & \\
\hline $\mathbf{5}$ & $\mathbf{2 5 . 4 0}$ & Brown w/ gray mottling, iron staining & & & \\
\hline & silty clay & & & \\
\hline & & & & \\
\hline & & & & \\
\hline & & & & \\
\hline & & & & \\
\hline & & & \\
\hline
\end{tabular}




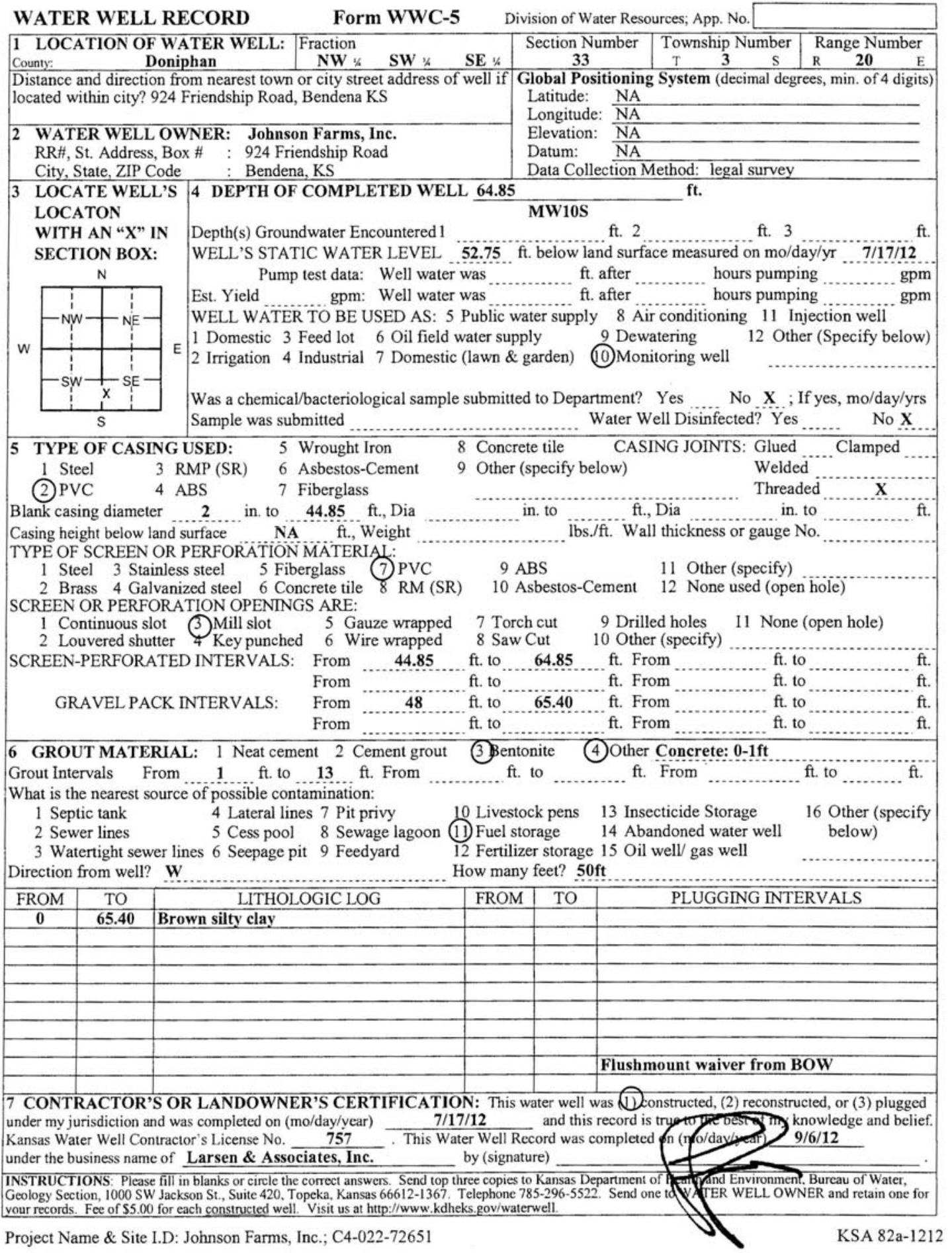




\section{Supplement 1:}

Titles and Leases for the Former CCC/USDA Facility and Adjacent Properties 
Titles 


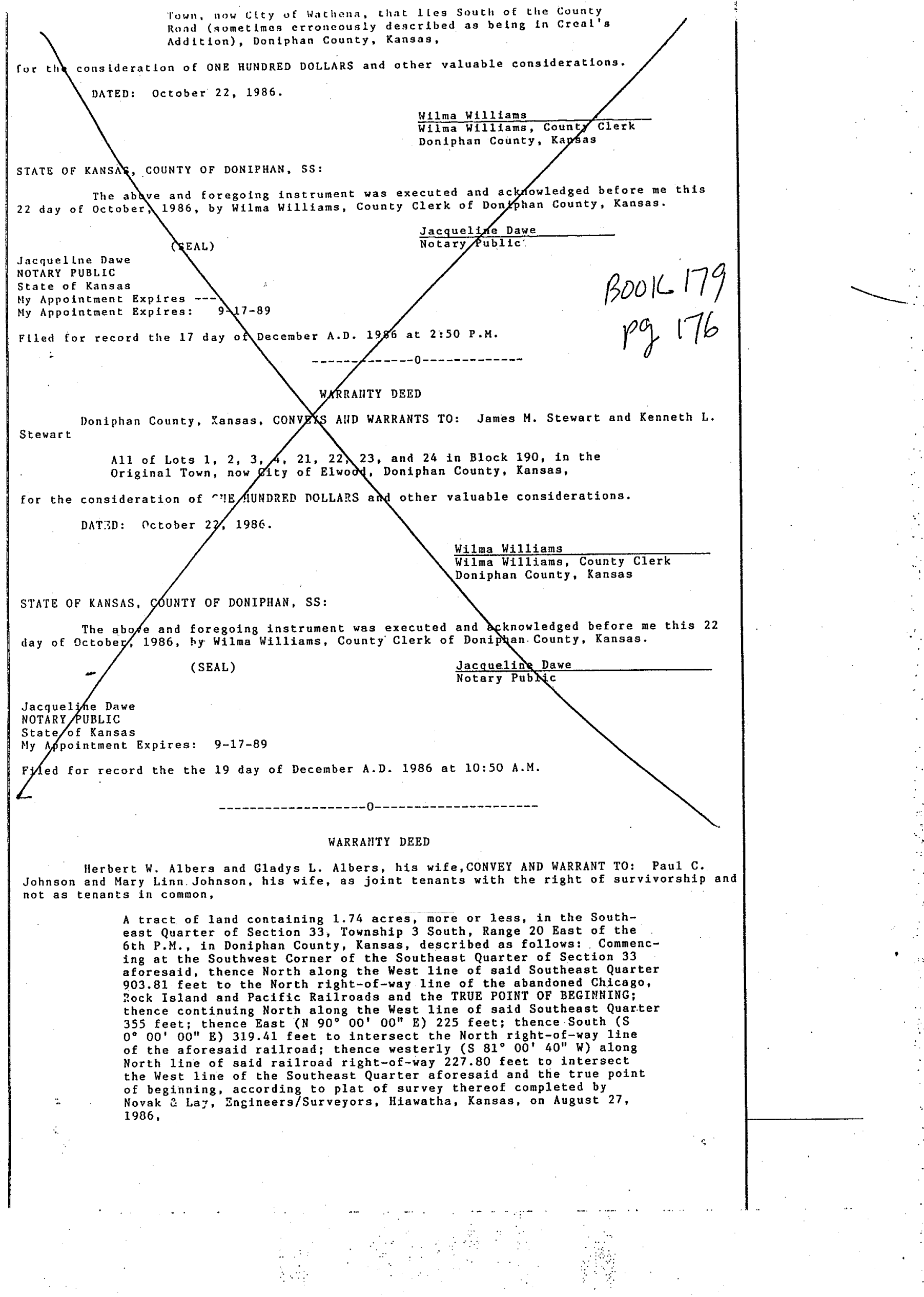




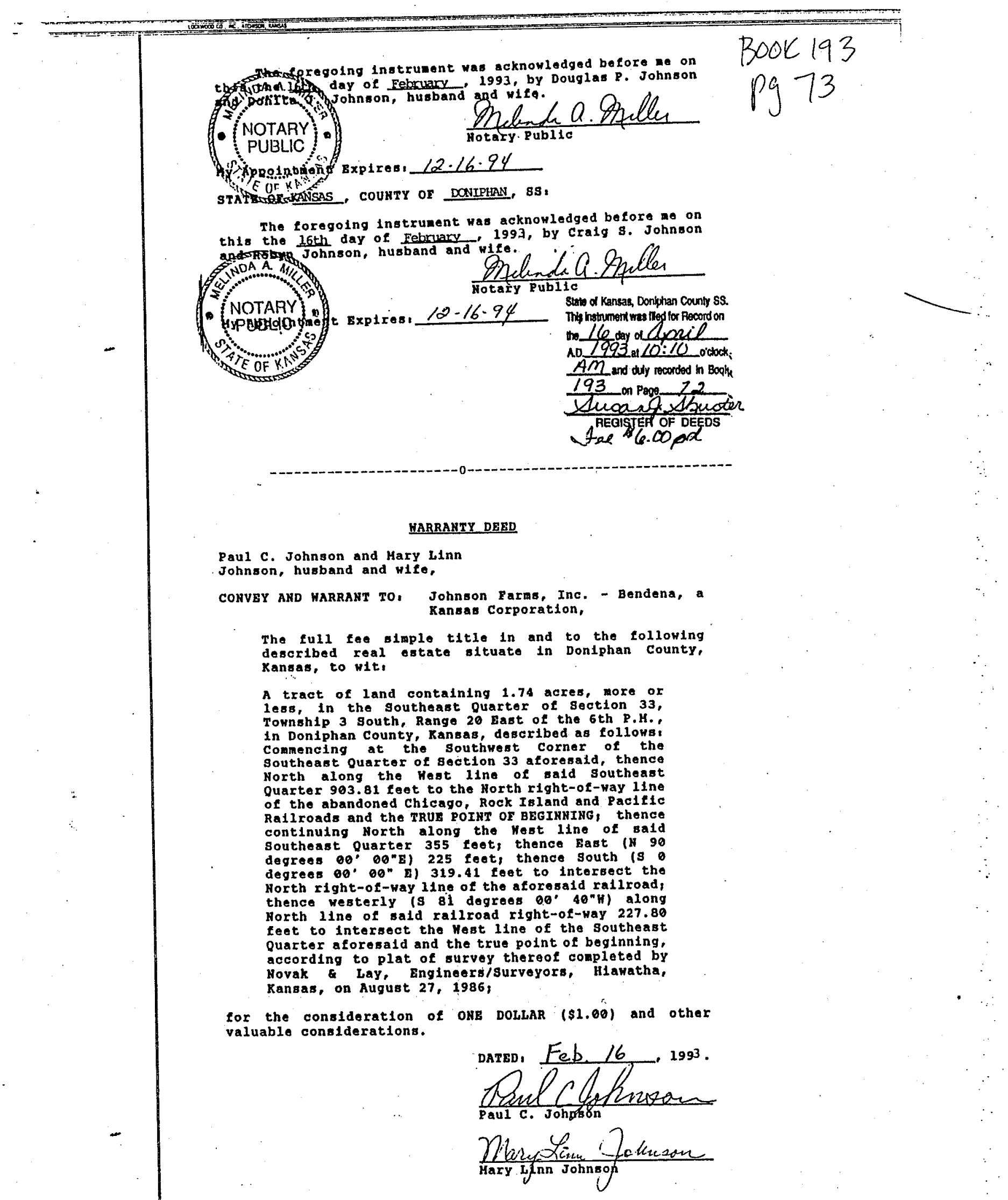


Leases 


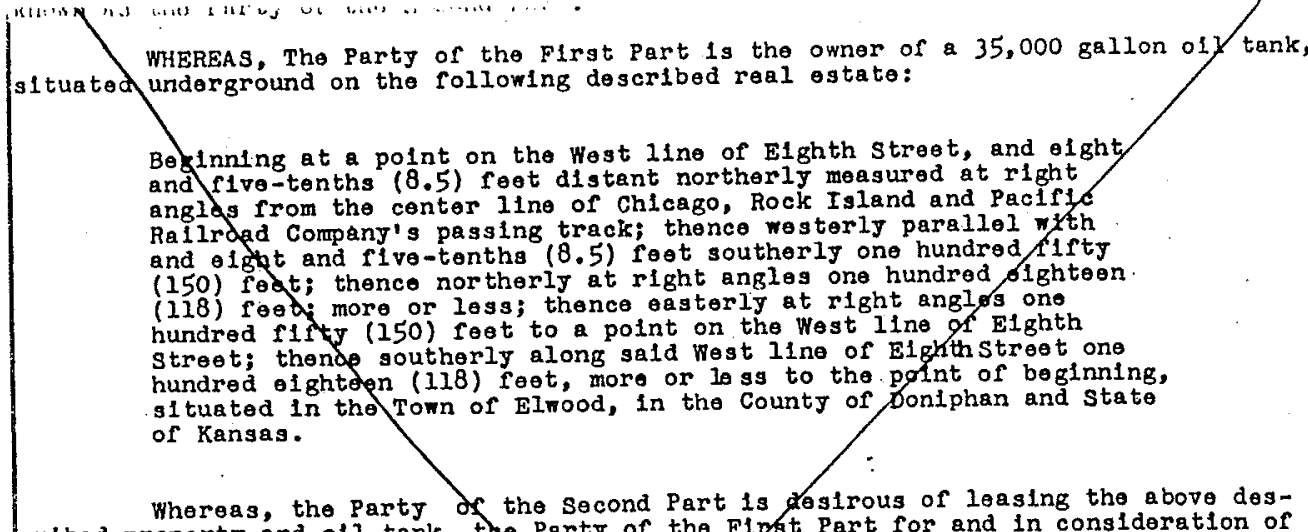
crlbed property and oll tank, the Party or the Fly to him pald by the Party of the One Dollar $(\$ 1.00)$ and other value to the Party of the segond Part sald deseribed property for a perlod of one jear from the date df this lgase.

It is hereby agreed by the Paxty for the First Part that the Party of tho second Part shall have the option to respr this lease at the explration thereor for an additional period of one year.

\section{Witness our hands this 17 th day of Xay, 1954.}

Cash 011 Company

STATE OF MISSOYRI COUNTY OF BUCHANAN SS:

(CORP)

By Bob sutt1e

(SEAL)

Party of tho First Part

Reriners Potroloum Compant

by Q. H. Saas, Secy

party of the Sacond Part

Subscribed and sworn to before me a Notary Public within and the county of Buchanay, state of Missour1, this 26 th day of May, 1954.

My Complission Explres May 7, 1955

(SEAL)

Filed for record the 26 day of May A. D. 1954 at 2 P. M.

U. S. DEPARTMENT OF AGRTCULTURE

AGRCULTURE STABTITZATION AND CONSERVATION

COMMODITY CREDIT CORPORATION

LEASE OF PROPERTY

mHIS LEASE, made and entered into this 1st day of May, 1954, by and botween

W. C. Albers of Bendena, Kanas, Lessor, and Comodity crealt Corporation, Lesser.

WITNESSETH THAT:

1. The Lessor leases to the Lessee, and the Lessee hereby leases from the Lessor upon the terms and conditions herelnafter stated, the following described real estate (hereinafter called "property") situated in the county of poniphan and atate of Kanses:

Tract in $\sin ^{2} 33-3-20$

$310^{\prime} \times 194^{\prime}$

containing 13 acres, more or loss.

2. The term of the lease ghall be for a perlod of 5 years, commenolng the 1st day of May, 1954, and ending the lst day of May, 1959, with the right of the tesseo, during such term or any extension thereof, to terminate sald lease, and ilablitty for any further rent, on the - day of May of any year, by glving 30 days previous notice in writing to the Lessor. 
3. As rent for sald property, the Lessee shall pay the Lessor - Dollars (\$103.50) per year, such rent to be payablo In advance, but to be apportionablo in tho event the loase is terminated as provided in paragraph 2 hereof:

4. The Lessor warrants that he is the owner of the property, has the right to give the Lesseo possession under this lease, and rill, so long as this lease remalns in effect, warrant and defend the Lessee's possession agalnst anj and all persons whomsoover.

5. The Lesses shall have the right, during this lease, to erect storage structures, or faclilties, make alterations, instali scalos, rences, or signs, in or upon the premises hereby leased and, at the explration of gald lease or any renewal or extension thereof or at anj time this lease is in erfect, may remove sald storage structures, faclifties, scales, fences, or signs or any part thereof, whether or not such structures, racliftios, scales, fences or slgns havo become legalis a fixture.

6. The Lessoe shall not assign this lease without the written consent of the Lessor. The Lessee, may, however, sublet the structures on the premises leased hereunder, or any one or more of them for the term of the lease or any part thereof upon such torms and conditions as Lessee may wish to so sublet.

7. The Lessee, if required by the Lessor, shall upon the explration of this lease, or reneral thereof, restore the premises to the same condition as that existing at the time of entering upon the same under this lease, reasonable and ordina - wear and tear and damages by the elements or by circumstances over which the Losseo has notcontrol excepted: Provided, horever, That if the Lessor requires such restoration, the Lessor shall give written notice thereof to the Lessee 30 dejs before the termination of the lease.

8. The Lessor grants and gives the Lesses the option as a consideration of this loase and for the further consideration of one dollar, the recelpt of which $1 \mathrm{~s}$ hereby acknowledged, to renew sald lease for a perlod of 5 years from the lessor, his helrs, executors, administrators, and assigns, for the sum of one Hundred three $\&$ $50 / 100$ Dollars $(\$ 103.50)$ per jear.

\section{9. (Marked out on orlginal)}

10. In the event anj increased tax assessment is made against the Lessor or the property by virtue of the erection of storage structures and facilities thereon by the Lesseo, the Lessor agrees to cooperate fully in any contest of such increased assessment which the Lessee foels should be made. The Lesseo agrees that the rental hereunder shall be adjusted uprard by the amount of any such increased tax assessment which the Lessor and Lessee mutually agree to be proper or which is determined to be legally valid in court procerdings.

11. No member of or Delegate to Congress or Resident Commissioner, shall be admltted to any share or part of this lease or purchaso or to any benefit that may arise therofrom, but this proplsion shall not be construed to extend to this leaso or purchase if mado with a corporation for its general benefit.

12. The Lessor warrants that he has not emplojed anj person to sollc1t or socure this lease upon any agreement for a commission, percentage, brokerage, or contingent foo and that no such consideration or paynent has been or wil be made. Breach of this warranty shall give CCC the right to annul the lease, or, in its discretion, to deduct from the rental or purchase price the anount of such comission, percentage, brokerage or contingent fees. This warranty shall not apply to conmlsalons payable by the Lessor if the lease is secured or made through a bona-fide agent maintained by the Lessor for the purpose of leasing or seliling his property.

(Seal) W. C. Alberg. IESSOR COMMODITY CREDIT CORPORATION, LESSE

(To be reproduced In ASC stato orfice) By I. H. Libol

Chairman, Doniphan County Asc Committeo

State of Kansas, Donfphan County, ss.

BE IT REMEMBERED, That on this 26 th day of May A. D. 1954 before me, the undersigned, a Notary Public in and for the County and State aforesaid, came W. $C$. Albers to personaliy known to be the same person who executed the within instrument of writing, and such persons duly acknowledged the execution of the same.

IN WITNESS WHEREOF, I have hereunto set my hand and affixed my Notarial seal, on the day and jear last above written.

My Commisgion expires 5-23-54

(SEAL)

N. V. Wakeman

Filed for record the 26 day of May A. D. 1954 at $3: 30$ P. M. 
recolded in Vol. $S$ at $P$ age 16 in the records of the Probate Court of Doniphan County, Kenge that the sald Katherine The owned a lifo ostate in the following dercribed real estate, to wit:

The Eat Halr of the Northwest Quarter of the Northwest Quapter except 20 feet for road way, and also the Northesst Quarter of the Southwest Quarter of the Northwest Querter, except the Zast 20 feet and also, tha East 20 feet of the Northwest Querter of the Southwest Quarter of the lorthwest Quarter, all in Section 17, Township 3, Range 21. Dontphan County, Kansas.

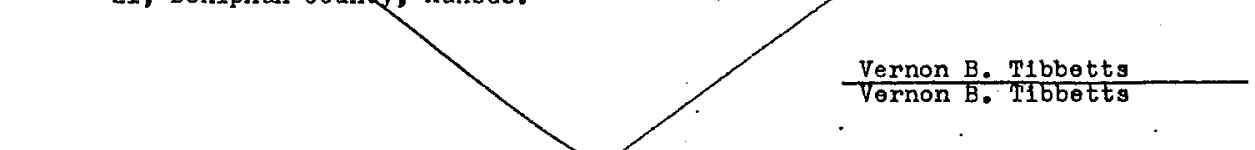

STATE OF KANSAS DONIPHAN COUNTY SS:

Be It remembered that on this 17 th das of Apr11, 1959 , before me the undersigned, a Notary Publio in and for the County and state aforesald came Vernon $B$. T1bbetts who is personaliJ knosn to me to be the same person who signed the within and foregoing affidavit and who was then by me duly byom as to the truth of the statements therein contained and who then duly acknowledged his oxecution of the same for the purposes and intents therein expressed.

In witness hereor I have hereunte placed my hend and notarial seal, the day and jear last abope stated.

Comm. Exp: $160.5,1960$

(SEAL)
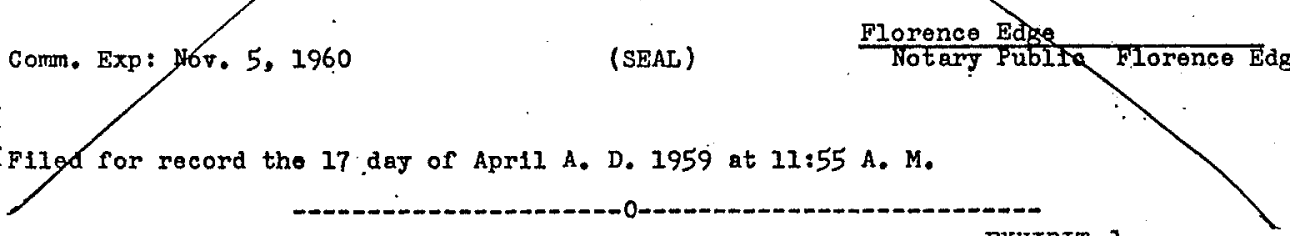

EXTENSION OF LEASE

$$
\underset{(7-G R)}{\text { EXHIBI }}
$$

"This extension of Lease made and entered into this 28 day of April, 1959 , by and between W. C. Albers, herelnafter called Lessor, and CommoditJ Credit Corporation, herelnafter alled Lessee. .

\section{HITNESSETH}

WHEREAS, The parties hereto have heretofore entered into a certain Leaso deted May 1, 1954, wherein the Lessor leased to the Lessee the following described property.

Tract in the SW corner of the sWl of 33-3-20 along road directiy

- North of the 'Eendena Elevator $3101 \mathrm{X} 194$

for a term onding May 1, 1959, and

WHEREAS, it is desirod by tho parties horeto to extend the sald loaso for an additional term under the same terms and conditions:

NOW THEREFORE, It is mutualiy understood and agreed by and between the parties hereto as follows:

(1) The sald Loase $1 \mathrm{~s}$ hereby extanded for an additional term beginning May 1 , 1959 and ending May $1,1964$.

(2) Lessors Grant and give to the Lesseo the option of further renewal of the lease for a additional perlod of five (5) yoars onding May 1 , 1969 under the same terms and conditions of the lease as extended herein provided the Iessee gives the Lessors written notice to ronow at loast thirty $(30)$ days prior to the time the lease, as extended, would otherwise explre.

IN WITNESS WHEREOF, THE partios hereto have executed this extension of Lease on the day first above written.

W. C. Albors
Lessor
Myrtle R. Albors
Lessor's Spouse
COMMODITY CREDTT CORPORATION

NOTE: It $1 \mathrm{~s}$ necessary to have the above agreoment acknowlodged and rocorded.

I, George D. Glark do hereby cortify that W. C. Albers and Myrtlo R, Albers, to me known to be the person (or Persons) who oxocuted the foregoing instmument, personaliy appeared before me and acknowledged that he (she or they) executed the same as his (her or their) freo act and deed and, in case said instrument was oxecuted the same as his (her or thelr) froe act and deed and, in cose said instmument was executed on behalf of. a corporation, that he (she or the g) as

(Insert neme of offlcer(s) and his (her or thalr) offlalal titio(s) Thame of Corporation) was (wore duly authorlzed by the Board of Directors of sald 
corporation to oxecute the said instmument on bohalf of sald corporation and to afflx the corporate soal theroto.

Given under my offlelal hand and soal this day of Apr11 28, 1959,

My Conmission Explros Julg 13, 1961.

(SEAL)

$\frac{\text { George D. Clark }}{\text { Notary Pribl10 }}$

Filed for record the 28 day of April A. D. 1959 at 1:45 P. M.

LEASE

7 THIS LEASE; made and entered into th1s 24 day of April 1959, by and between Mr.D. M. Tucker \& Mrs. B. A. Tucker, Husband \& Wife, of Doniphan County, Kens. hereinafter called tho Lessors; and the Ged. Wi Kerford Quarry Coa; a Delawaro Corpopation, $415 \mathrm{Uthh}$, Atchison, Kansas, hereinafter called the Lesseo.

KITNESSETH :

Ledgors, for and in conslderation of the sum of one dollar and other valuable consideratide, recolpt of which is hereby acknowledged, and of the covgrants and agreements on the part of the Lesseo hereinafter contalned, hereby grayt, demise, lesse and let to sald Lessee, for the purpose of exercising the exclusive right to mine, quarry an remove rock, to bulld roads and structures, and to orect such spoil

banks as are reatonably necessary in the opinion of the Lesseo to produce, save, and
take care of sald products, all that certain tract of Land describodas allows, to wit

B1ocks 85, 8ो, 99, 100, 101, 102, 103, 104, 105, 106, 120, 121, 122,

Inclusive, al in the town site of Palermo, Doniphan County, Kansas.

The lessee shall bay to the Lessors, or thelr order as full compensation for the rights and priviloges herein granted, and, for all/rock quarried and removed by the Lesseo, a royalty of eight cents per ton for all rock quarrled and disposed of by the Lessee off the preslises. The amount of such royalty, to be pald by the Lessee, shall be determined bf the duly authorized agents or inspectors of any gover.mont, person, corporation, or d ther parties, to whom ans such rock may be sold or delivered, and, such measurements shall be conclusive/upon the parties hereto in the computation of royalt1es payable hereunder. The lessoe shall make payment of the rojalties, herein reserved, monthl, on or before the twenty-fifth day of each month for all rock quarried and removed fom the premisef during the preceding calendar month.

The Lesser shall have the right of ingross to and egress from the premises herein above described, for the conduct of quapry operations, and for the transportation of rock therefrom, with the right) at their option, to orect cruahing equipment, to move in machinery and all equipmant/as found to be necessary for prosecution of business, to constmuct and operate a raylroad spur track to the quarry, and, any such track, machinery, structures and Impropements placed thereon by the Lesseo, incldent to such operations, shall remain pertonal property of the Lessee, and may be removed by the Lesseo.

This lease shall commence on the 24th dax of April, 1959, and ahall continue for a perlod of one year. The Lessors expressly gtant, to the Lessee, the right, privilege and option to renew and extend this leash and contract for - successive sdditionel periods of one joar oach sald renewal odtion and privilege is to be considered as automatically exerc1 fod on each successive renewal dato unloss Lesseo gives a thirty day notice, in wrifing, before the explpation of the then existing term, of 1 ts desire and intent to cancel, and, the giving of notioe shali cancel this lease. The malling of such notice by Registered United dtates Mall, to the Lessors, their successors of assigns, at the address last known to the Lesseo, shall be suffictent notice hereunder.

Lessee shall. Indernglfy and forever hold Lessors blampless for injurles or damages of any kind whatsoover, (but not to the leased premlshs), wh1ch may arise out of; in connection with, of, as a result of this lease or Lessel's operations on sald premises under this lease, and, Lessee shell and covenents and agrees to malntain, at ali times, during the $\mathrm{I}_{\mathrm{f}} \mathrm{e}$ of this 10 ase and contract, public 11 bility insurance in gmounts not less than $\$ 100,000$ dollars for personal injuries in. anj one accident, $\$ 50,000$ dollars for $/$ injury to any one person (Including death,) and $\$ 25,000$ doliars for property damagep.

Lessors bereby agrees and warrant that they have a good and vald title to the property heyein described, and, that they will warrant and defend some unto said Lessee, ifs hoirs and assigns.

Lesoors further agree that Lessee shall have the right, at any timd to redeem for Zessors, by payment of mortgages, taxes or other liens, on the alpre descrlbed land, in the event of def ault of payment by Lessors, and be suborgated to the rights of the holder thereof.

It is furthor agreed that during the term of this lease, there shall acchue, to the Lessors, a minlmum annual royalty of $\$ 100$ dollars per year, which shall

appl on earned rojalty payments, when an as such rovalty payments become due, oif rogelty payments, whether annual minimum or earned, shall be construed as

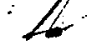


. The Lessor grants and gives the Lessee the option as a consideration of this lease and for the further consideration of one dollar, the receipt of whicb is heroly acknowledged, to renew sald lease for a perlod of 5 years frum tho Lesfor, his helr, executors, administrators, and assigns, for the sum of two hundred Dgdiars $(\$: 00.00)$ per year.

\section{Marked out}

10. In the event any increased tax assessment is made agelinst the Lessor or the property by virtue of the erection of storage structures and racilities therea by the Lessee the Lessor agrees to cooperate fully in any contest of such increased asgessment whifh the Lessee feels should be made. The Lesser agreef that the rental -hereunder shalf be adjusted upward by the amount of any such incroased tax assessment which the Lessof and Lessee mutually agroe to be proper or which is deternined to be legally valid in court proceodings.

11. Lo member of or Delegate to Congress or

Besident Commissioner, shall be admitted te any share or part of this lease or pypchase or to any benefit that may arise therdfrom, but this provision shall not by construed to extend to this lease or purchase if made with a corporation for its geferal benefit.

12. The Lessor warrants that he has not employed any person to solicit or secure this lease upop any agreement for a commssion, percentage, brokerage, or contingent fee and that if such consideration or payment has been or wiII bo made. Breach of this warranty shall give CCC the right to annul the lease, or, in its discr etion, to deduct from the rental or purchase p 1 ce the amount of such commission, percentage, brokerage, or cdntingent fees. Thls warranty shall not apply to conmissions payable by the Lessor if the lease is gecured or made through a bona-fide agent maintalned by the Lesisor for be purpose of leasing or selling his property.

(Seal) Fred B. Smith, LESSOR Fred $\overline{3}$, Smith

(Seal) Merle Smith, Lessor Merle Sinith

\section{Loinel W. Seana, Witness}

Lolnel W. Sesna

I, Leroy Hodder, do hereby certify that Fred B. Smith \& Merle Smith, COMMODITY CREDIT CORPORATION, LESSEE

$$
\text { By } \frac{\text { Robt. A. Myers }}{\text { Robt. A. Myers }}
$$

Chairman, Doniphan ASC County Committee Contracting officer

be the person (or persons) who executed the foregoing instrument. to me known to peared before me and acknowledged that he (she or they) executed the same as his (her or thelr) free act and deed and in case said instrument was executed on behalf of a corporation, that he (she or they) al kobt. A. Myers, Chairman

ASC County Committios insert name of officer(s) and his her or theip) official title(s) (Name of Corporation)

authorlzed by the Board of Directors of sald corporation to execute the said instrument on behalf of ald corporation and to affix the corporete seal thereto.

Given under my official hand and seal this day pf July 26, 1963.

(SEAL).

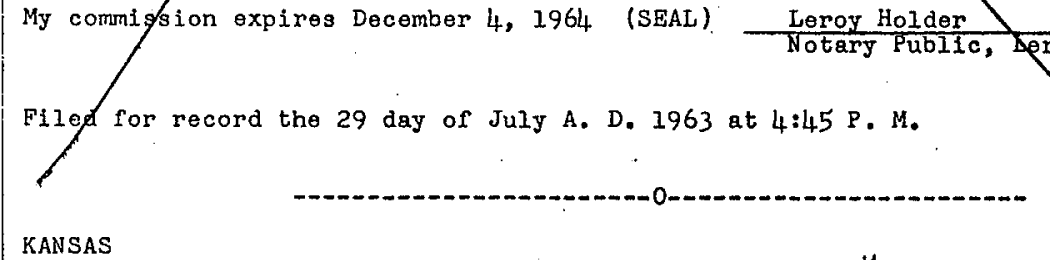

FORM K-1-BI-3

EXTENSION OF LEASE

"This Extension of Lease made and entered into this 22 day of July, 1963 by and between W. C. Albers, hereinafter called Lessor, and Commodity Credit Corporation, herelnafter called Lessee.

WETW WSSETH, Whereas, the parties hereto have heretofore entered into a certain lease dated May 1, 1954, wherein the Lessor leased to the Lessee the following described property:

Tract in the SW corner of the SW $\frac{1}{4}$ of Sec. 33-3-20 along road

directly North of the Bendena Elevator $310^{\prime} \mathrm{X} 194^{\prime}$

For a term ending May 1, 1959, and

Whereas, it is desired by the parties hereto to extend the sald lease for an additional term under the same terms and conditions: 


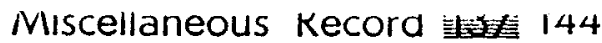

Now therefore, It is mutually understood and agreed by and between the

(1) The sald lease is hereby extended for an additional term beginning May 1, 1964, and anding May 1, 1969.

(2) Lessors grant and give to the Lessee the option of further renewal of the lease for an additional period of five (5) years onding May 1, 1974; under the same terms and conditions of the lease as oxtended hereln provided the lesseo gives the Lessors written notice to renew at least thirty (30) days prior to the time the lease, ex extended, would otherwise expire.

Lease on the day first above written.

W. C. Albers
W. C. Albers Lessor
Myrtle Albers
Lessor's Spouse, Myrtle Albers
COMMODITY GREDIT COHPORATION

I, Edward Swinford, do hereby certify that W. C. Albers \& Myrtle Albers, to me known to be the person (or persons) who executed the foregolng instrument, personally appeared before me and acknowledged that he (she or they) executed the same as his (her or their) free act and deed and, in case said instrument was executed the same as his (her or their) free act and deed and, in case sald instrument was executed on behalf of a corporation, that he (she or they) as Robt. A. Myers

Chairman ASC County Committeo (Insert name of Officer(s)
Has duly authorized
and his (her or their) official titlo(s) (Name of Corporation)

by the Board of Directors of said corporation to execute the said instrument on behalf of said corporation and to affix the corporate seal thereto.

Given under my official hand and seal. this day of July 22, 1963, my commission expires Jan. 26, 1967

$3-1-63$

(SEAL)

Edward Swinford

Filed for record the 29 day of July A. D. 1963 at $4: 45$ P. M.

KAISAS

FORM $\mathrm{K}-1-\mathrm{BI}-3$

\section{EXTENS ION OF LEASE}

"This Extension of Lease made and entered into this 25 day of July, 1963 by and betheen Ralph C. Moser, hereinafter called Lessor, and Commodity gredit Corporation, serelnafter called Lesse日.

WITHESETH. Whereas, the parties hereto have heretof hre entered into a certain lease dated Sept. 16, 1954, wherein the Lessor leased to the Lessee the rollowing described preperty:

Approximatel 56,000 square feet. A strip 160 feet wide on the West side, $S E_{4}$ fec. 19-3-21, containing 7.3 acres, more or $10 s$. For a term ending Datober 1, 1959, and

Whereas, it is desired by the partyes hereto to extend the said lease for an additional term under the same terns and conditions:

Now therefore, it is mutually understood and agreed by and between the parties hereto as rollows:

(1) The said lease is hereby extended for en additional term beginning October 1, 1964, and ending Octobor 1,1969

(2) Lessors igrant and give to the Lessee the optidy of further renewal of the lease for an additfonal period of five (5) years ending Octaber 1 , 1974 , under the same terms and/conditions of the lease as extended herein provided the Lessee gives the Lessoys written notice to renew at least thirty (30) deys prior to the time the lease, gs extended, would otherwise expire.

In fitiness whereof, the partios hereto have executed this Extension or Lease on the day first above written.

Ralph C. Moser

Ralph C. Moser, Lessor

Mrd Ralph C. Moser

Mra. Ralph G. Moser, Lessor's Spouse
COMMODITY CREDIT GORPORATION

By Robt. A. Myers Contracting Offlcer, Robt.A. Myers

$=$ 


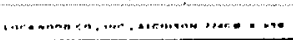

$R C-51+363^{\prime \prime} A^{\prime \prime}-343-K$

DECLARATION OF EXPIRATION OF MINERAL RESERVATION

KNOW ALL MEN BY THESE PRESENTS: That Whoreas in that certain special farranty deed deted the 30 th day of December, A.D. 1947, Between the Federal Land Bank of Wichilta, Wichita Kansas, a corporation, as First Party, and Amor \& Hattlo E. Schaurflor, HeW,

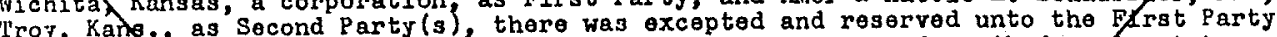
an interest in the minerals in, upon and under the following described repl estate gituated in the County of Doniphan and State of Kans.., to-wit:

Twp 2 South, Rge 21 East

Section 30: South 50 acres of the NE$\frac{1}{4}$

to which deed referance is hereby made for a more particular description of the interest in the minerald so reserved.

Now, lHEREFORE the First Party states that there was no production of minerals from said premises and that the premises were not beyig developed or operated at the end of the primary term of said reservation, and tyat such reservation has expired and is of no further force and effect.

ITWESS the signature of the Bank this 3rd gay of March, 1969.

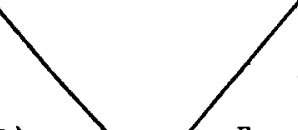

IHE FEDERAL LAND BANK OF WICHI'AA, Wichita, Kansas, a corporation,

ATPEST:

\section{(CORP SEAL)}

ron T. Rowe11

By F.L. Buellesfeld

Byron T. Rowell, Kssistant Secretary

STATE OF RANSAS

COIIIY OF SEDGWICK

SS.

Before mo, the undersigned, a Notary Public in and for said County and State, on this 3rd day of. March, $\not 969$, personally appeared F.L. Buellesfeld, to me personally known and known to me to fe the identical person who subscribed the name of the maker thereof to the foregoing instrument as 1 ts Vice President, and he being by me duly sworm did say that he $/ \mathrm{s}$ such officer and that the seal affixed to said instrument is sworn did say that the same was signed and sealed in bethe corporate seal of said corporation and that the same wath corpordion by authorlty of its board of directors, and he acknowledifed to me that he execated the same as his free and voluntary act and deed, and as the freo and volumtaz act and deed of such corporation, for the uses ald purposes set forth and specified therein.

WITyess my hand and seal the day and year last above written.
(SEAL)
Hy coptmisaion expires: $4-2-72$
Filód for record the 17 day of March A.D. 1969 at 9 A.M.
KANSAS
FORI K-I-BI-3

TIIS RXTEHSION OF LEASE made and entered into this 13 day of March, 1969, by and between W.C.Albers Estate, Herbert W. Albers, Executor, hereinafter called Lessor. and Commodity Credit Corporation, hereinafter called Lesseo. WITNESSETH

WHEREAS, the parties hereto have heretofore entered into a certain leasë datela May 1 , 1954, wherein the Lessor leased to the Lesseo the following described property: (Enter legal description of the property)

$$
\begin{aligned}
& \text { Tract in the SW corner of the SWt of Sec. } 33-3-20 \\
& \text { along road directly North of the Bendene Elevator } \\
& 310^{\circ} \times 194^{\prime}
\end{aligned}
$$

for a term ending April 30, 1974, and

WHEREAS, It is desired by the parties hereto to extend the sald lease for an additional term under the same terms and conditions:

NOW, THEREFORE, it is mutually understood and agreed by and between the partios heroto as follows:

(1) The said lease is hereby extended for an additional term beginning May 1,1969 , and ending Apri1 30, 1974 .

(2) Lessors grant and give to the Lessee the option of further renewal of the lease for an diditional perlod of five (5) years ending April 30 , 1979, under the same temin and conditions of the lease as extended here197, rovided the Lessee gives the Lessors written notice to renew at leas thirty (30) days prior to the time the lease, as extended, would otherwise expire. 
IN WINNESS WHEREOF, the parties hereto have executed this Extension of Lease on the day rirst above written.

(Lessor's Spouse)

W. C. Albers Estate

Herbert W. Albers, Executor (Lessor) Herbert W. Albers, Exceutor

COMMODITY CREDTT CORPORATION

By Robert A. Myerg

ChaIman Doniphan 1 SC County

Committeo, Robert A. Myers

\section{ACKNO WLEDGMENT}

I, Hillman Hull, do hereby certify that Herbert W. Albers, to mo known to be the person who executed the foregoing instrument, personaliy appeared before me and acknowledged that he executed the same as his pree act and doed and, in case said instrument was executed the same as his free act and deed and, in oase said instrumen was executed on behalf of a corporation, that he (she or they) as Robert A. Myers, Chairman Doniphan ASC County Committee

(Insert name of officer(a) and his (her or their) official title(s)(Name of Corporation) was duly authorized by the Board of Directors of said corporation to execute the said instrument on behalf of sald corporation and to affix the corporate seal thereto.

Given under my official hand and seal this day of 13 th of March, 1969,

(SDAL)

Hillman Hull

Notary. Public

Hiliman Iluli

Troy, Kansas 66087

My Commission expires October 12, 1972

Filed for record the 28 day of March A.D. 1969 at 9 A.M.

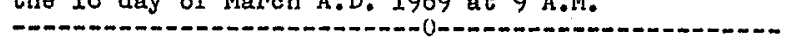

DEDD

.$<$

- IPIS DEED, Made this 21 st day of February, 1969, between UNIO/ PACIFIC RAILROAD COMANY, a corporation of the State of Utah, party of the first part, and CIIY OF LLWOOD, municipal corporation of the State of Kansas, party of the second part:

\section{WTPKSSETH, That}

WIIEREAS the party of the second part desires to acglire an easement for street purposes, dyer, upon and along, and for the construction, maintenance and oper ation of water and pewer lines and other public utilities ander the surface of, the property hereinafter described; and

WHEREAS, the platy of the first part is willing to donate said easement to the party of the second pert for the purposes aforegaid;

NOW, THEREFORE, in consideration of the premises, the sald party of the firsf part does by these presents gtant, donate, conyey and confirm unto the said party of the second part, and unto its successors and psigns, a PERPEIUAI EASEMENT, for the purposes aforesaid, over, upon, long and under the surfece of the property of the party of the first part in the Colpaty of Dohiphan, State of Kansas, described as follows, to wit:

The south 66 feet of $\mathrm{he} N 1 / 2 \mathrm{NE} 1 / 4$, Section 36

Township 3 South, Rongd 22 East of the Sixth Principal

Meridian, Doniphan/Count), Kansas, except the west 460

feet thereof.

RESERVING, however, to the party of the first part, its successors and assichs, the right to construct at any and alI times and o maintain railroad tracks, pipe linfs and telephone and telegraph and electric power pole and wire lines over, under and across (but in such a way gs not unreasonably to inderfere with) said streot, water and sewer lines or other public utilities in the locabion above described, it being understood that the right so reserved to the party of the first part, its successors and assigns, is retainga along with the general right of he party of the first part, its successors and asfigns, to the use of the premises heresin described for any purpose not inconsistent with the use by the party of the second part of said easement for the purposes hprein defined.

This egsement is made subject to all outstanding superior rights, including those for highrays and other roadways and rights of way for irrigation and drainage ditches, pipe lines, pole and wire lines, and the right of renewals and extensions of the same

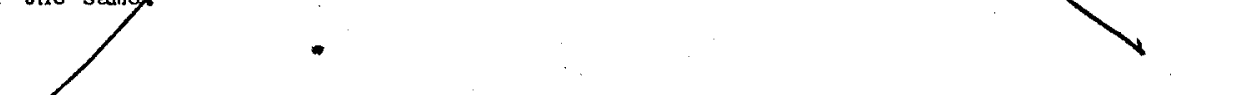


WHEREAS, Commodity Credit Corporation, herelnafter called "CCC," as the lessee, leased from W. C. Albers, as lessor, pursuant to an Instrument dated May 1 , 1954, hereinafter called "the lease," a tract of real estate located in Doniphan County, Kansas that was erroneously described in the lease as follows:

$$
\begin{aligned}
& \text { Tract in SW } 1 / 4,33-3-20 \\
& 310^{\prime} \times 194^{\prime} \text { contalnting } 11 / 2 \text { acres, more or less; }
\end{aligned}
$$

AND WHEREAS, the lease was recotded In the office of the Register of Deeds of Doniphan County, Kansas, in Book 133 on page 557 and was extended and renewed fro time to time as shown by instruments recorded in the office of the Register if Deeds of Donlphan County, Kansas, In Book 137 on page 466, in Book 144 on page 574, and In Book 152 on page 487 ; and

WHEREAS, the tract of land leased by CCC was erroneously described in the lease and all extenstons and renewals thereof, and sald tract should have been described in the lease and all extensions and renewals thereof as follows:

$$
\begin{aligned}
& \text { Tract in the Southwest Quarter of the } \\
& \text { Southeast Quarter of Section } 33-3-20 \\
& \text { along the road directly north of the } \\
& \text { Bendena Elevator consisting of a tract. } \\
& \text { measuring } 310 \text { feet by } 194 \text { feet and con- } \\
& \text { taining } 1 / 2 \text { acres, more or less; }
\end{aligned}
$$

AND WHEREAS, the lease and all extensions and renewals thereof have been terminated and are of no force and effect and same should be released and discharged

NOW THEREFORE, Comodity Credit Corporation disclaims, releases, and discharges any and all interest in the real estate located in the State of Kansas and in Doniphan County, which it acquired under the lease and the extensions and renewal thereof, pursuant to Instruments recorded in the records of Doniphan County, Kansas in Book 133 on page 557 , in Book 137 on page 466, in Book 144 on page 574, and in Book 152 on page 487 . This instrument $1 \mathrm{~s}$ executed on behalf of CCC pursuant to dele gation of authority published in the Federal Reglster (26 FR 3900).

COMMODITY CREDIT CORPORATION

By: Frank A. Mosier State Executive Director Kansas State ASCS Office

\section{ACKNOWLEDGMENT}

STATE OF KANSAS

COUNTY OF RILEY SS.

On this 19 day of Oct, 1973, before me, the undersigned Notary Public in and for the county and state aforesaid, personally appeared Frank A. Mosier, State Executive Director, Agricuitural Stabilization and Conservation Service, United States Department of Agriculture, who is personally known to me and personally known to be such State Executive Director, and the same person who executed the within and foregoing instrument, and he duly acknowledged the execution of the same for and on behalf of and as the free act and deed of Comodity Credit corporation.

IN WITNESS WHEREOF, I have hereunto set my hand and affixed my official seal the day and year last above written.

\section{(SEAL)}

Corrine 1. Kanode

\section{CORRINE L. KANODE}

NOTARY PUBLIC

Riley County, Kansas

My Commission Expires

January 19,1976

My comission explres Jan 19, 1976

Filed for record the 24 day of October A.D. 1973 at 9:00 A.M.

\section{ADDENDUM TO LEASE}

The-existing Lease dated August 31, 1972, between the undersigned Lessor and Lessee is hereby amended to include the following additfonal paragraphs:

1. To secure certain borrowings, Lessee may mortgage Its leasehold intere ft

hereunder by mortgage or deed of trust to Cont inental I11inols National Bank and Trust Company of chicago or 1 ts nominee (the "Cont inental Bank"). In the event of any default by lessee in the performanco 6 any of Its obligations hereunder, Lessor will give written notice to the Contirental Bank at the following address:

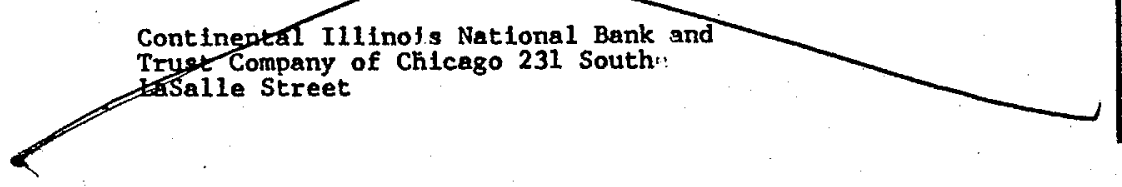




\section{Railroad Right-of-Way Abandonment}


Commencing one thousand forty-one and eighty hundredths feet North of the Southwest Corner of the Southeast

Duarter of Section Twenty-three (23). Township Two (2)

South, Range Nineteen (19) East to a point on the centerline of U.S. 36 HIghway; running thence easterly along the centerifne of U.S. 36 Highway a distance of one thousand two hundred seventy-one and thirty hundredths feet to point;

Thence South at right angles to the centerline of the U.S 36 Highway sixty feet to the South right-of-way ine of sald highway for a point of beginning:

Thence on an angle of $3^{\circ}$ to the left three hundred fifty feet to a half inch iron bar:

Thence on an angle of $17^{\circ} 20^{\prime}$ to the left one hundred

sixty-five feet to a half inch iron bar;

Thence on an angle of $73^{\circ} 20^{\circ}$ to the left three hundred

fifty feet to a half inch iron bar;

Thence northerly on an interior angle of $95^{\circ} 00^{\prime}$, folis

Hindred thirty-two and fifteen hundredths feet to the

Sdith right-of-way line of said U.S. Highway 36 ;

Thence westerly two hundred two feet along said South

right-of-way line to a spike and a washer.

Thence northerly at an exterior angle of $89^{\prime} 28^{\prime}$, sixty

feet afong said right-of-way 1ine; Thence/westerly along

sald right-of-way line two hundred thirty-one and forty

hundredths feet to the point of beginging.

for the sum of one Dollar (Sf.00) and other good and valuable consideration.

EXCEPT AND SUBJECT TO: Grantok John 5 . May, by $A$ special warranty above, releases his 1 ien on the money judgment obtained for his client fh Case No. $77 \mathrm{C} 68$ in the District Court of Doniphan County, Kansas, which was later converted by Sheriff's Sale to an interest in the real estate above described, upon the condityon that such lien immediately attach to the proceeds of this conveyance. This special wax ranty is also expressly 11 inited to the warranty that grantors have not permitted or llowed any other encumbrance to affect the title now held by Bill N. Glenn as recelver of The Industrial Loan and Investment Company of Sedalia, Missouni during the time that grantors may havg had an interest in the bbove real estate.

Dated December 1, 1981

STATE OF KANSAS, Atchison/COUNTY, ss

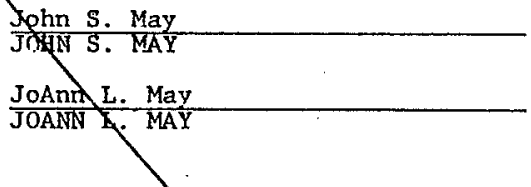

BE IT REMEMBERED, That on this 1st day of Decentier A.D. 1981, before me, the undersigned, a Notary Public in and for the County and State aforesaid, came John S. May and -JoAnn L. May, his wife who/are personally known to me to be the same persons who executed the within instrument of writing and such persons duly acknowledged the execution of the same.

last above WTites wh

IN WITNES 8 WHEREOF, I have hereunto set my hand and afixed my seal, the day and year

last above written.

(SEAI)

Amelia R. Ostergard
AMELIA R. OSTERAARD

AMELIA R. OSTERGARD

Atchis on/County, Ks

My Appt. Exp. July i6, 1982

Filed for record the 7 day of January A.D. 1982 at $3: 45$ P.M.

\section{OQUITCLAIM DEED}

KNOW ALL MEN BY THESE PRESENTS That WILLIAM M. GIBBONS, Trustee of the Property of the CHICAGO, ROCK ISLAND AND PACIFIC RAILROAD COMPANY, Debtor, as Trus tee and not as an individual Grantor herein, pursuant to Court Order No. 234, dated May 5, 1980, entered by the United states District Court for the Northern District of Illinois, Eastern Division, in the matter of the Chicago, Rock Island and Paciffc Raflroad Company, Debtor, No. 75 B 2697, and pursuant to every other power and authority to him pertaining, in consideration of the sum of TEN

DOLLARS $(\$ 10,00)$ and other good and valuable consideration, the receipt whereof is hereby acknowledged, does hereby OUITCLAIM and CONVEY subject to the reservations and exceptions hereinafter set forth, unto EDWIN DENNIS FORD and CAROLYN KAY FORD as Tenants in Common, herein designated as "Grantee," all of Grantor's right, title and interest, estate, clain and demand, if any, in the following described parcel of land situated in the County of Doniphan,

State of Kansas, to wit:

Being a rectangular tract of land lying and located in

the West Half of the Southeast Ouarter of Section 33,

Township 3 South, Range 20 East, Doniphan County, Kansas,

more particularly described as follows:

Commencing at the southwest corner of the Southeast Quarter of Section 33; thence North $90^{\circ}$ East along the south line of 
said section for a distance of 30 feet, more or less, to a point; thence North $0^{\circ}$ Fast for a distance of 1,015 feet, more or less, to a point on Grantor's southerly right-of-way line as is presently evidenced, monumented, or recorded in Bendena, the POINT OF BEGINNING: thence North $81^{\circ} 30^{\circ}$ East, more or less, along Grantor's southerly right-of-way line for a distance of 4.40 feet more or less, to a point; thence North $0^{\circ}$ East for a distance of 175 feet, more or less, to a point; thence South $81^{\circ} 30^{\circ}$ West, more or less, and parallel with the center1ine of Grantor's track for a distance of $\mathbf{4 4 0}$ feet, more or less; thence South $0^{\circ}$ East for a distance of 175 feet, more or less, to the POINT OF BEGINNING; said parcel being the west 440 feet of that interest granted to the C.K. \& N. Railway Company by John Albers and W1fe, by deed recorded in Book 21, Page 352, and a 25-foot strip of land adjolning said west 440 feet, being the south 25 feet of that interest granted to the C.K. \& N. Rallway Company by John Albers and Wife by deed recorded in Book 21 , Page 410 , sald parcel containing 1.77 acres, moreor less.

The Grantor reserves unto himself, his successors and assigns, all of the codil ofl, gas, casinghead gas, Iigntte, sulphur, water and all other ores and minerals of every kind and hature in addition to the foregoing, underlying the surface of the property herein described. together with the full right, privilege and license at any and all times to explore or drill For and to protect, conserve, mine, take, extract, remove and market any and all such products

The frantor reservè for himself, his successors and assigns, an easement for the continued maintenance, operation and use of all existing driveways, roads, conduits, sewers, water mains, gas Iines, electric power lines, communication 1ines, wires and other utilities and easements of any kind whatsoever on said'premises, whether or not of record, whether owned operated, used or maintained by frantor, the Grantor's 1icensees or others, with the reasonable right of entry for the purpose of repalring, reconstructing and replacing same; and the (rrantor further reserves unto himself, his successors and assigns, the right and privilege to convert existing. 11censes, leases and agreements, for driveways, roads, conduits, sewers, water mains, gas IInes, electric power ifnes, wire and other utilities to permanent easements bv issuance of a sultable grant in recordable form.

The frantor reserves for himself, hls successors and assigns, a perpetual easement on, over and across that portion of the property represented by hatched lines and designated "Right-of-Way Easement" on Exhibit "A" dated October 28, 1981, attached hereto and by reference made a part hereof, including, but not limited to, the right to maintain, repair, renew ube and operate any track or tracks and appurtenances now existing upon or across the property herein described and to conbtruct, maintain, repair, renew, use and operate any additional track or tracks and appurtenances across the property herein described, and also the right to insta 11, maintain, use and operate wires or cables, together with poles or other structures supporting the same above the surface of said property, or pipes or conduits or other structures beneach the surface of the property.

The Grantee, its successors and assigns, covenant and agree that it shall neither do nor cause to be done any act that will unreasonably impede the flidw of drainage water over the property herein described so as to adversely affect rail operations. This covenant shall in no way be construed to prohibit the frantee from erecting buildings or other improvements on the property herein described, provided that the drainage equivalent to that existing at the time of this conveyance is maintained, whether naturaliy or by other means. This covenant

sha11 run with the land and be binding upon the frantee, its sucdessors and assigns.

The frantor further reserves unto himself, his successors, grantees and assigns, exclusive perpetual easements for the installation, maintenance and use of pipelines, telephone, radio, radar or laser transwilssion systems, wires, fibers, conduits, utility and energy transmission lines of every kind and character with all necessary supporting structudes and devices which may be constructed or installed on, under, over, across and along any portion of the property herein described lying within fifty feet of the centerline of the main railroad tracks as now existing and represented on Exhibit "A" dated October 28, 1981 . Such installation, maintenance and use shall not interfere with the Grantee's use of the surface. The failure of frantor, his successors, grantees or assignees to exercise easement rights hereby reserved prior to January 1,1991 shall in no event be construed to be a nonuser causin. said easements to terminate, but if Grantor shall fall to convey or assign to others the easement rights hereby reserved by January 1, 1991, then the easements reserved in this paragraph shall automatically cease and determine.

Notwithstanding any other provision of this instrument, the Grantor sha11 in no event incur liability to the Grantee for failure of or defect in the title or estate of the Grantor in and to the property herein described.

This conveyance is made pursuant to the terms of a Purchase Agreement dated September 1, 1981 and the terms thereof shall survive delivery of this Quitclaim Deed. October, 1981 .

IN WITNESS WHEREOF, this instrument is executed by the Grantor this 30 day of WILLIAM M. GIBBONS, Trustee of the Property of the CHICAGO, ROCK ISLAND AND PACIFIC RAILROAD COMPANY, Debtor

WITNESS :

WIIIIam M. Gibbons 
APPROVED

K. B. Nelson

General At torney

STATE OF ILLINOIS)

COUNTY OF COOK ) SS

On this 30 day of October, A.D., 1981, before me, the undersigned, a Notary Public in and for said County, in said State, personally appeared WILLIAM M. GIBBONS, Trustee as aforesaid, to me known to be the identical person named in and who executed the for egoing instrument and acknowledged he executed same as his voluntary act and deed.

(SEAL)

Mary C. Peloso

My Commtssion Expires: May 22, 1984

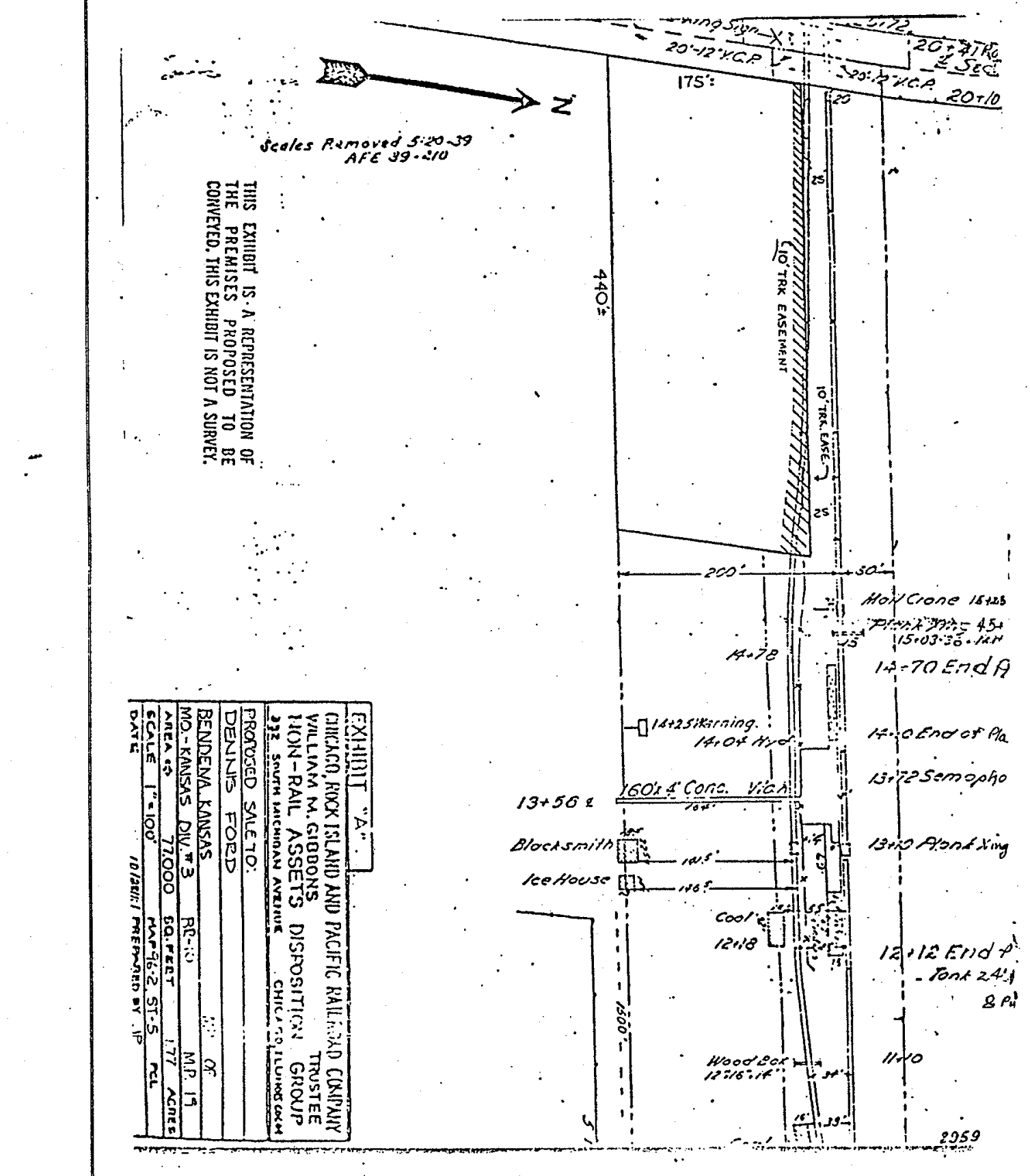

Filed for record the 13 day of January A.D. 1982 at 12:45 P.M. 


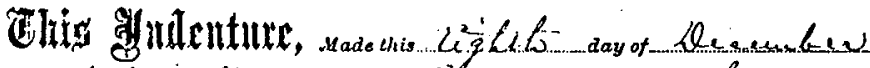

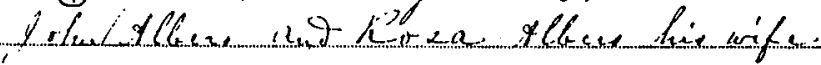

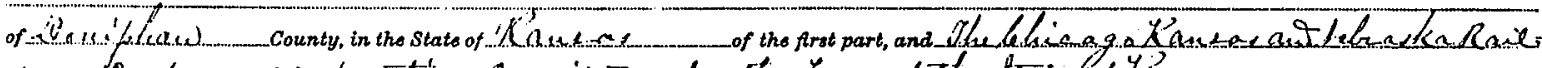

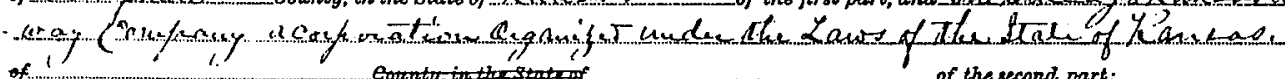

ZHlitutgstlh, That said part ise of the first part, in consideration of the sume of

............................

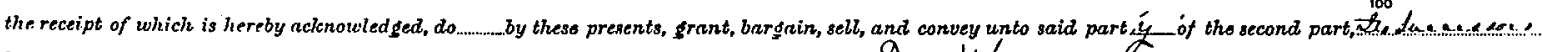

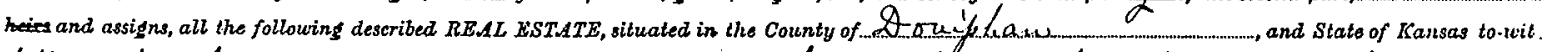

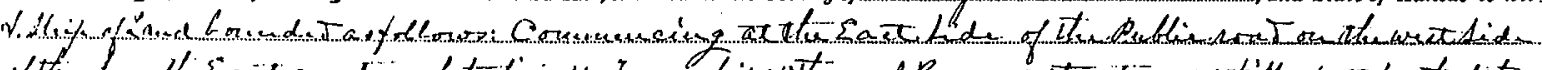

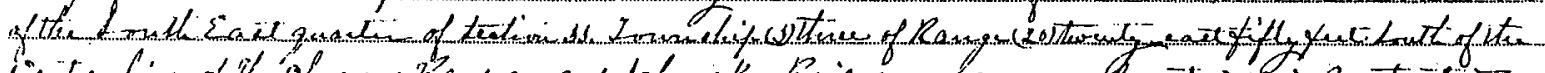

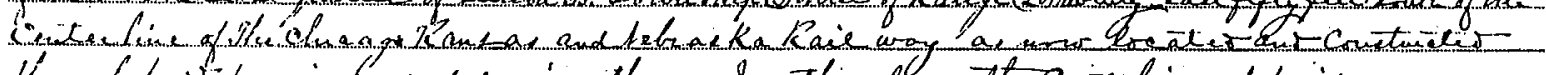

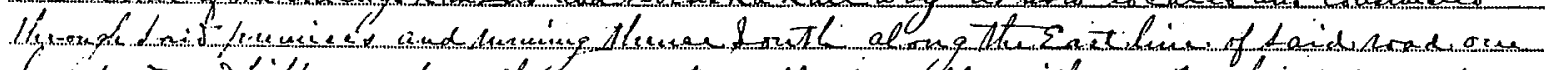

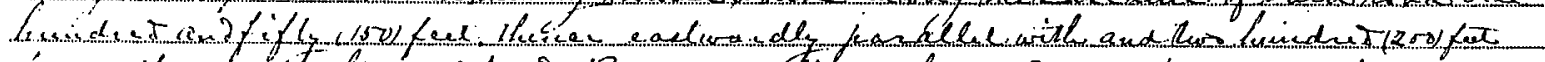

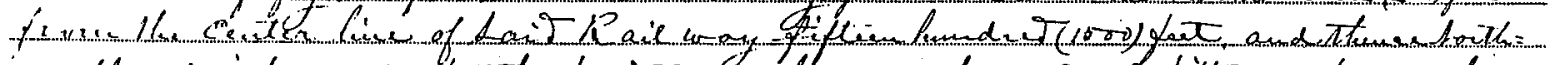

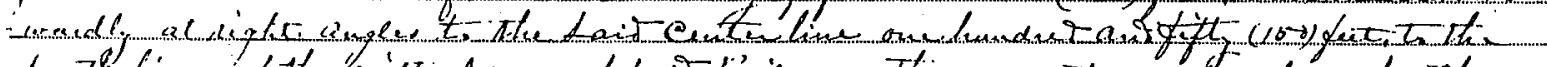

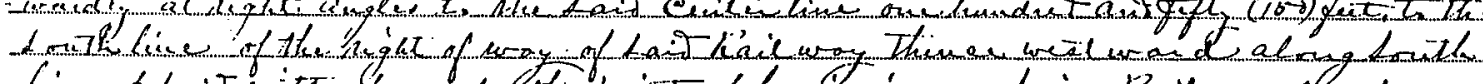

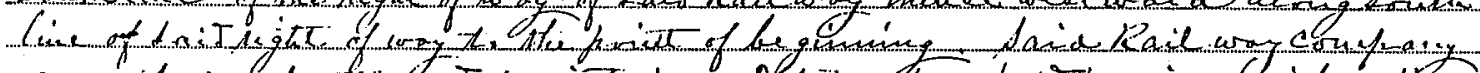

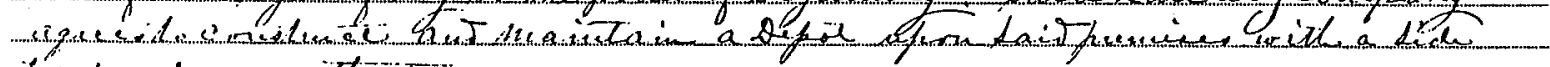

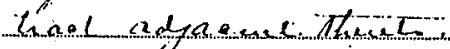

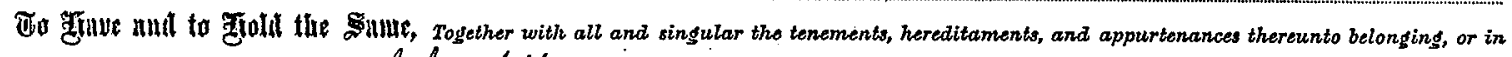

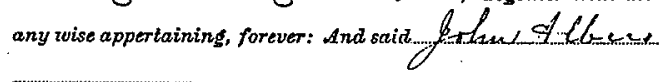
exfectors, or administrators, do . . hereby covenant, promise, and agree, to and with said part.y of ihe second part, that at the detivery of these presents

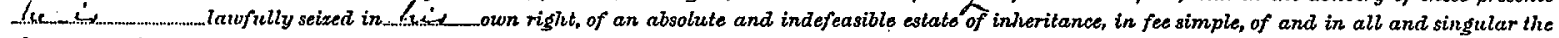

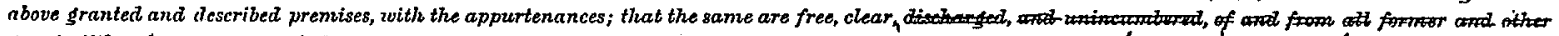

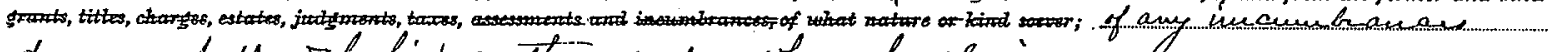

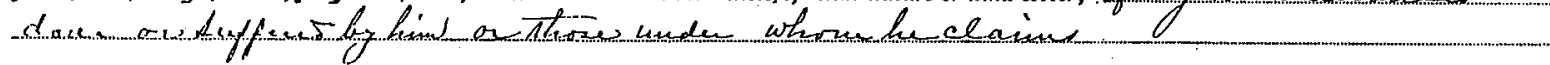

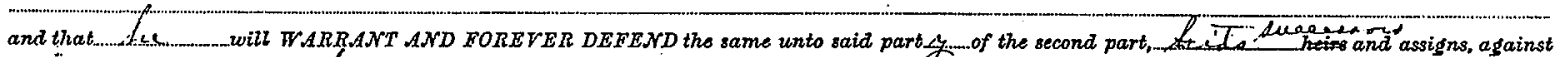

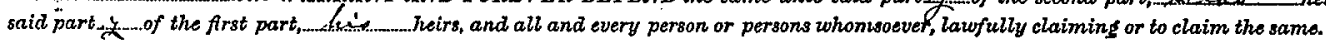

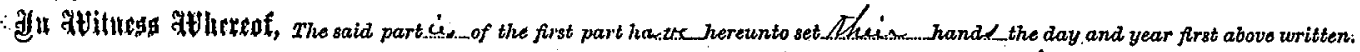

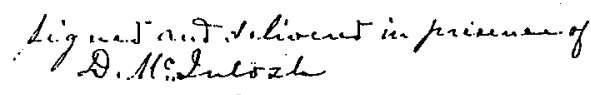

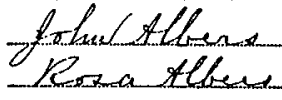

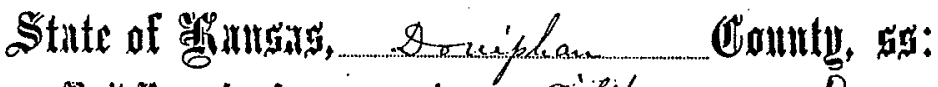

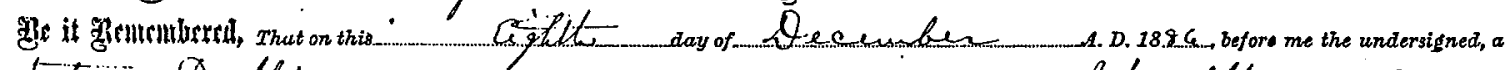

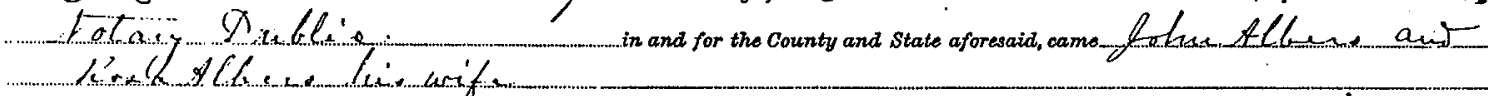

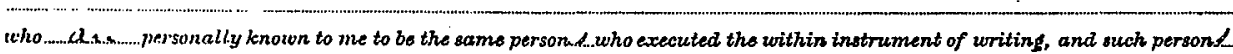
the exceution of the same.

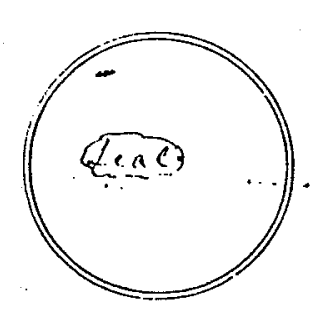

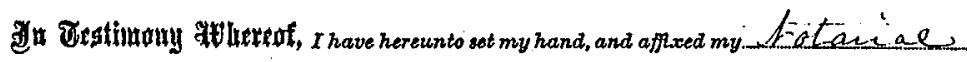
seal, the day and year last above written.

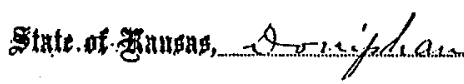

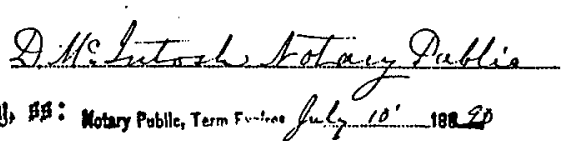

I hereby certify the foregoing to be a full, true and perfect copy of the original instrument which was filed with mee for

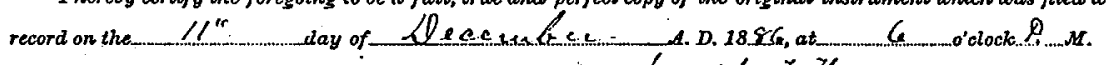

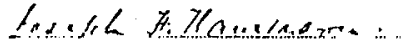




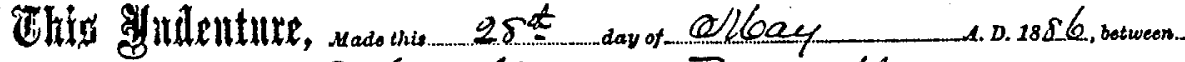

Folim alber and Rexa albers.

Slaubaud \&ed \&efo

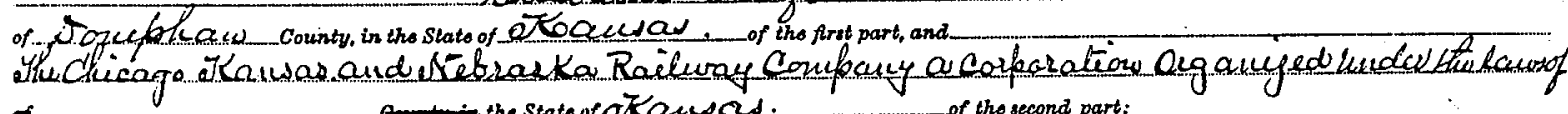

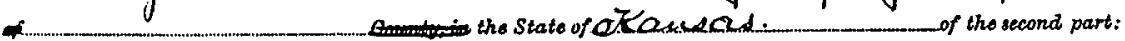

Ittitutssth, That said mart $L$ ies of tha first part, in consideration of the sum of

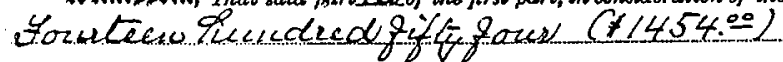

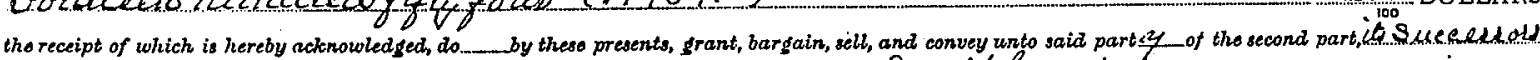

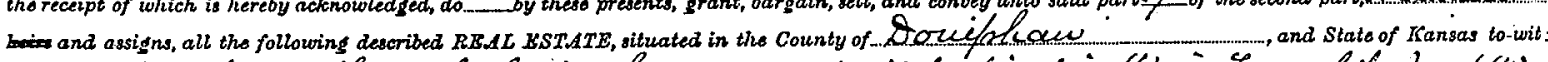

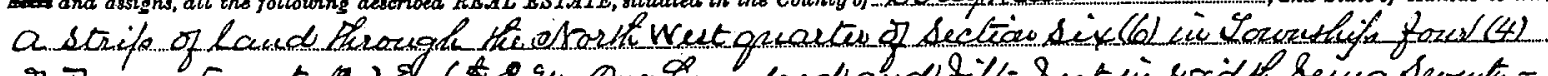

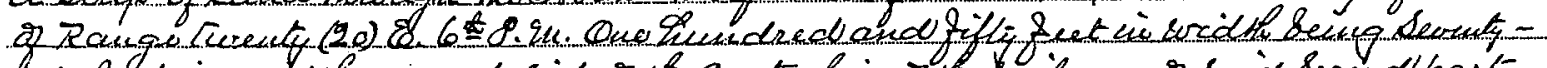

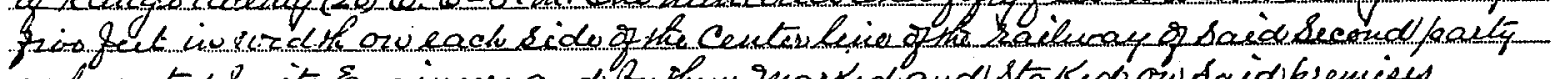

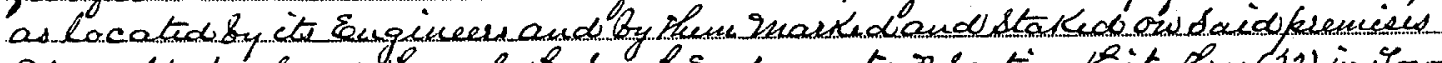

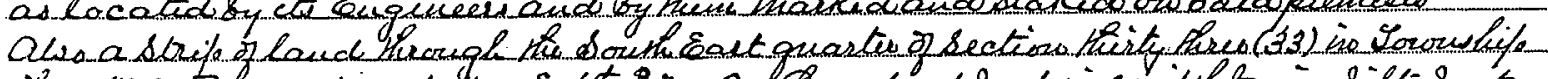

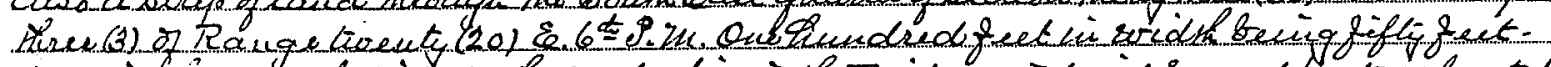

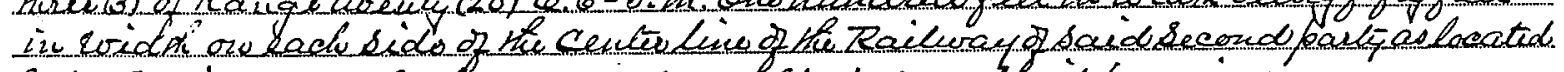

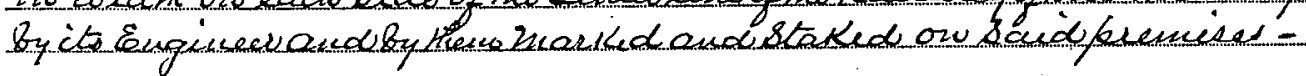

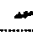

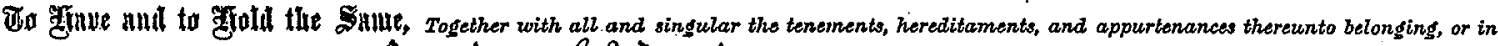
any wiso appertaining, forever: snd said \&aetie of he first fart

wectors, or administrators do - Terehy covenant promises and asree, to and with sidd partef of the second part, that at the delivery of these presents Her and and in all and singular the above granted and described premises, with the appurtenances; that the same are free, ilear,

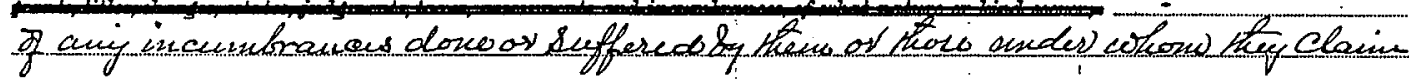

and that the - will WARRAST AND FOREVER DEFEND the same unto said party of the second part, ins SAsceessolu and assigns, against said part Lis of the first part, ffecis. . heirs, and all and every person or persons whonssoever, lawfully olaiming or to olaim the samo.

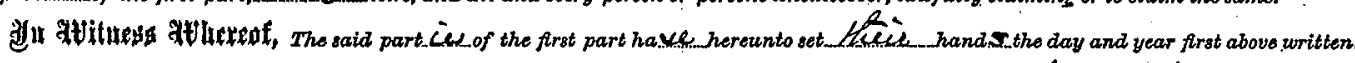
Tolm celbers

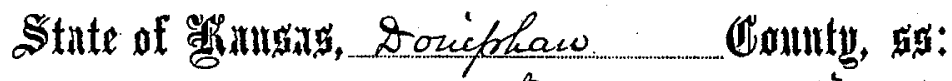

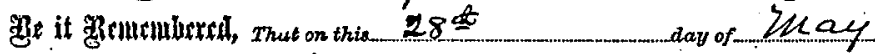
QA - - tayey of elele

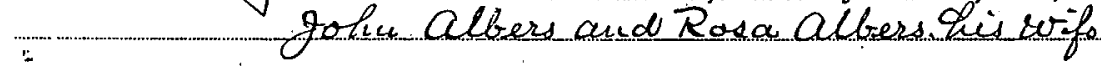

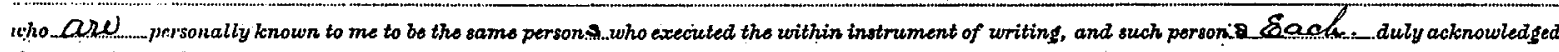
the execution of the same.

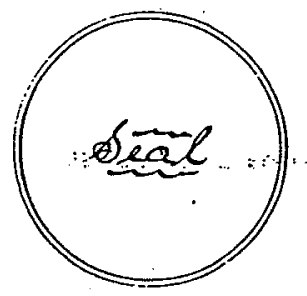

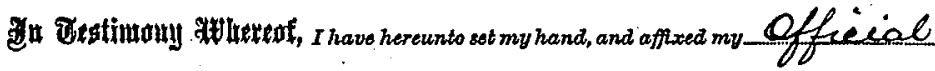
seal, the days and year last abovo writtem.

State of :

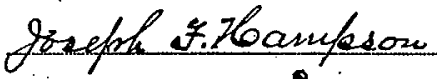

I hereby certify the foregoing to be a full, true and perfect copy of the original instrument which was filed with mo for

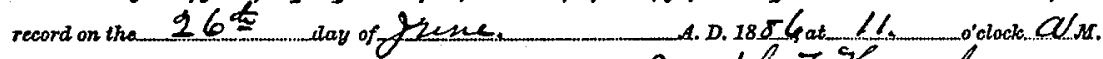

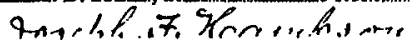


KNOW ALL MEN BY THESE PRESENTS That MILLIAM M. GIBBONS, Trustee of the Broperty of the CHICAGO, ROCK ISLAND AND PACIFIC RAILROAD COMPANY, Debtor, as Trustee and not as an individual Grantor herein, pursuant to Court Order No. 234, dated May 5, 1980 , entered by the United States District Court for the Northern District of Illinols, Eastern Division, in the Matter of the Chicago. Rock fsland. and Pacific Railroad Company, Debtor, No. 75 B 2697, and pursuant to every other power and authority to him pertaining, in consideration of the sum of TEN DOLLARS $(\$ 10.00)$ and other good and valuable consideration, the receipt whereof is hereby acknowledged, does hereby QUITCIAIM and CONVEY, subject to the reservations and exceptions hereinafter set forth, unto CONSUMER OIL COMPANY, INC., herein designated as "Grantee," all of Frantor's right, title and interest, estate, claim arid demand, if any, in the following described parcel of land situated in the County of Doniphan, State of Kansas, to wit:

Being a rectangular tract of land $1 \mathrm{y}$ ing and located in

the West Ha1f of the Southeast Quarter of Section 33 .

Township 3 South, Range 20 East, Doniphan County,

Kansas, more particularly described as follows:

Commencing at the southwest corner of the Southeast

Ouarter of Section 33; thence North $0^{\circ}$ East for a

distance of 1,015 feet, more or less, to a point on

Grantor's southerly right-of-way line produced as is

presently evidenced, monumented, or recorded in Bendena;

thence North $81^{\circ} 30^{\prime}$ East, more or less, along Grantor's

southerly right-of-way line for a distance of 470 feet,

more or less, to the Point of Beginning; thence North

$81^{\circ} 30^{\prime}$ East, more or less, a long said right-of-way

line for a distance of 120 feet, more or less, to a

point; thence North $00^{\circ}$ East for a distance of 175 feet, more or less, to a point; thence South $81^{\circ} 30^{\prime}$ West, more or less, parallel with Grantor's north right-of-way line for a distance of 120 feet, more or less, to a point; thence South $00^{\circ}$ East for a distance of 175 feet, more or less, to the Point of Beginning; containing .482 acre, more or less.

The Grantor reserves unto himself, his successors and assigns, all of the coal, oil, gas, casinghead gas, lignite, sulphur, water and al1 other ores and minerals of every kind and nature in addition to the foregoing, underlying the surface of the property herein described, together with the full right, privilege and license at any and all times to explore or drili for and to protect, conserve, mine, take, extract, remove and market any and all such products.

The Grantor reserves for himself, his successors and assigns, an easement for the con-

tinued maintenance, operation and use of all existing driveways, roads, conduits, sewers,

water mains, gas lines, electric power lines, communication lines, wixes and other utilities and easements of any kind whatsoever on said premises, whether or not of record, whether owned, operated, used or maintained by Grantor, the Grantor's Iicensees or others, with the reasonabic right of entry for the purpose of repairing, reconstructing and replacing same; and the Grantof further reserves unto himself, his successors and assigns, the right and privilege to convert existing licenses, leases and agreements, for driveways, roads, condults, sewers, water mains , gas lines, electric power lines, wire a of a suitable grant in recordable form.

The grantor reserves for himself, his successors and assigns, a perpetual easement on, over and across that portion of the property represented by hatched Iines and designated "Right-of-Way Easement" on Exhibit "A" dated June 12, 1981 and attached hereto and by reference made a part hereof, including, but not limited to, the right to maintain, repalr, renew, use and operate any tract or tracks and appurtenances now existing upon or across the property herein described and to construct, maintain, repair, renew, use and operate any additional tract or tracks and appurtenances across the property herein described, and also the right to install; maintain, use and operate wires or cables, together with poles or lother structures supporting the same above the surface of said property, or pipes or conduits or other structures beneath the surface of said property.

The Grantee, its successors and assigns, covenant and agree that it shall neither do nor cause to be done any act that will unreasonably impede the flow of drainage water over the property herein described so as to adversely affect rail operations. This covenant sha11 in no way be construed to prohibit the Grantee from erecting buildings or other improvements on the property herein described, provided that the drainage equivalent to that existing at the time of this conveyance is maintained, whether naturally or by other means. This covenan shal1 run with the land and be binding upon the Grantee, its successors and assigns.

The Grantor further reserves unto himself, his successors, grantees and assigns exclusive perpetual easements for the installation, maintenance and use of pipelines, telephone, radio, radar or laser transmission systems, wires, fibers, conduits, utility and energy transmission lines of every kind and character with al1 necessary supporting structures and devices which may be constructed or installed on, under, over, across and along any portion of the property herein described lying within fifty feet of the centerline of the main railroad tracks now existing and represented on Exhibit "A" dated June 12, 1981. Such installation, maintenance and use shall not interfere with the Grantee's use of the surface. The failure of Grantor, his successors, grantees or assignees to exercise easement rights hereby reserved prior to January 1, 1991 shall in no event be construed to be a nonuser causing said easements to terminate, but if Grantor shall fail to convey or assign to others the easement rights hereby reserved by January 1, 1991, then the easements reserved in this paragraph shall automatically cease and determine.

Notwithstanding any other provision of this instrument, the Grantor shall in no event incur liability to the Grantee for fidlure of or defect in the title or estate of the Grantor in and to the property herein described.

This conveyance is made pursuant to the terms of a Purchase Agreement dated september If, 1981 and the terms thereof shall survive delivery of this Quitclaim Deed. 
1981.

WITNESS:

Tricia Lee Brown

Títle \& Closing officer

Approved

K. B. Nelson

STATE OF ILLINOIS)

COUNTY of COOK ; SS

On this 18 day of November, A.D., 1981, before me, the undersigned, a Notary Public in and for said County, in said State, personally appeared WILLIAM M. GIBBONS, Trustee as aforestid to me known to be the identical person named in and who executed the foregoing instrument and acknowledged he executed isame as his voluntary act and deed.

(SEAL)

My Commission Expires: May 22, 1984

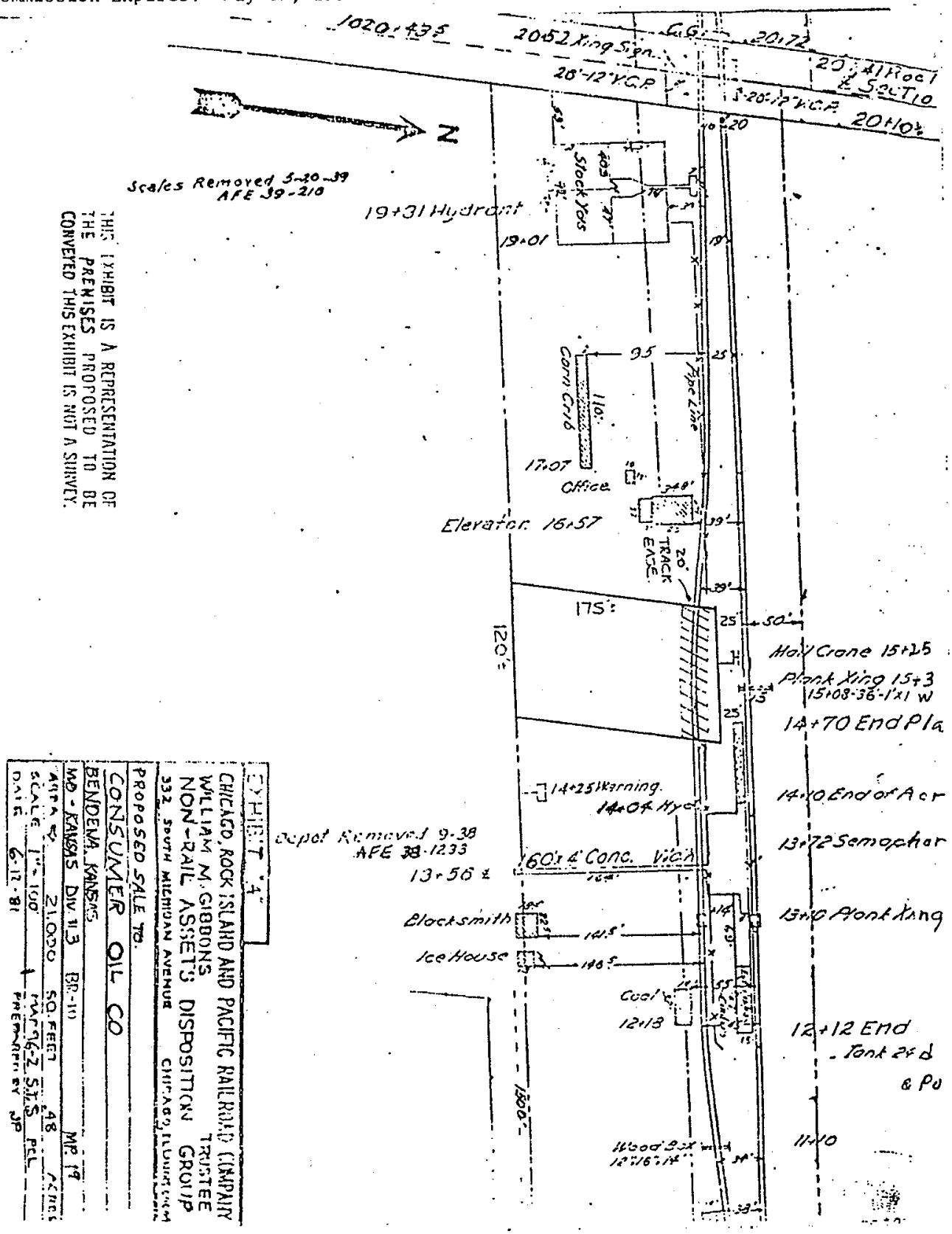

WILLIAM M. GIBBONS Trustee of the Wroperty of the CHICAGO BOCK ISLAND AND PACIFIC RAILROAD COMPANY, Debtor

William M. Gibbons

Trustee 
State of Ransas

Dohiphan County

SS ;

BE IT REMEMBERED that on this 15th day of February, 1984 before me a Notary Public o the coupty and state aforesald, came Charles F. Gentet and Betty J. Gentet, husband and wife, who are gersonally known to me to be the same persons who signed the within and foregolng General Waxranty Deed and who then duly acknowledged their execution thereof.

stated. IN ITNESS WHEREOF, I have hereunto set $m y$ hand and seal the day and year last aboye

\section{THELMA HORNER}

Doniphan County, Ks

Commission expires: $1-\$ 2-87$

Filed for record the 15 dey of February A.D. 1984 at $3: 20$ P.M.

(SEAL)

\section{The lma Homer}

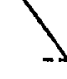

\section{QUIT CIAIM DEED}

THIS IPDFITURE, Made this 17th day of February, A.D. 1994, between RICHARD L. LANTER

and DONNA LOUISE LANTER, HUSBAND ANP WIFE, of Atchis on county, in the fstate of Kansas, of the first part. and DONNA LOUISE LANTER, FUSTEE OF THE DONNA LOUISE LANTER REVDCABLE I.IVING TRUST, of Atchison County, in the State of Rnsas of the second part:

VITNESSETY, That said parties of the first nart, in consideration of the sum of me and No/100 DOLLARS, the receipt of which is hereby acknowledged do by these presents, REMISE RELEASE AND QUIT-CLAIM, unto said party of the second nart, hey successors and assigns, all the following-described real estate situated in the rounty of Honiphan and state of yansas, to-wit:

The Southeast Quarter ( $\mathrm{SE}_{4}^{2}$ ) of Section Thirty-three (3.3), Township

Eour (4), Range Nineteen (19), Noninhay County, Kansas, and

The Southwest Quarter (SW

Four (4), Range Nineteen (19), Confaintng 32 ) acres more or less, Doniphan County, Kansas

The South Half of the Northwest/nutrter of Section 33, Township 4, Range 19, Doniphan County $;$ kansak SUBJECT TO, THE TERMS OF A BEVOCABLF AYVINI TRUST AGREEMENT DATFD
February 17,1984 .

TO HAVE AND TO HOLD THF SAMT, Tghether with all and singular the tenements, hereditaments and appurtenances thereun to belonging or in anywise dppertaining, forever.

IN NITNESS WHEREOF, The said parties of the first pdt have hereunto set their hands, the day and year first above writtey.

STATE OI KANSAS, ATCHISOY COUNTY, SS.

BE IT REMAMBKED, That on this 17 th day of February A.D. 1984. before me, the undersigned, Notary pubyAc in and for the County and State aforesaid, came RICHARD L. LANTER and DONNA LOUISE LANTER HUSBAND AND WIFF, who are personally known to me to be the same persons who executed the y/thin instrument of writing, and such persons duly ackndpledged the execution
of the same.

IN TESTIMONY WHEREOF, I have hereunto set my hand and affixed my seal, the day and year last abgue written.

\section{(SEAL) \\ My Appointment Expires: $1-28-85$}

Filg for record the 21 day of February A.D. 1984 at 9:00 A.M.

\section{Evelyn Lorenz}

Richard L. Lanter

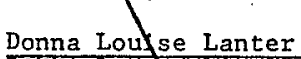

Donna Loulse Lanter

CORPORATTON QUIT-CLAJM DEED

THIS INDENTURE, made on the 13th day of February, A.D., One Thousand Nine Hundred and Eighty-four, by and between SCF, Inc., a corporation, duly organized under the laws of the State of Missouri, of the County of Jackson, State of Missouri, Party of the First Part, and Edwin Dennis Ford and Carolyn K. Ford, husband and wife, of the County of Doniphan, State of Kansas, Parties of the Second Part, (Mailing address of said first named grantee is Wathena, Xansas 66090).

WITNESSETH, that the said Party of the First Part, in consideration of sum of One Dollar $(\$ 1.00)$, to it in hand paid by the Parties of the Second Part, the receipt of which 
is hereby acknowledged, does, by these presents REMISE, RELEASE and FOREVER QUIT-CLAIM unto the said Parties of the Second Part, the following described lots, tracts or parcels of land, lying, being and situate in the County of Doniphan and State of Kansas, to-wit:

Commencing at the Southwest Corner of the Southeast OneQuarter (SEt) of Section 33, Township 3 South, Range 20 East of the Sixth Principal Meridian, Doniphan County, Kansas; thence North 0 degrees 00 minutes 00 seconds East (assumed bearing), along the west line of sald Southeast Ouarter, 650.63 feet; thence North 81 degrees 00 minutes 40 seconds East parallel to the center line of the Chicago, Rock Is1and and Pacific Rallroad, 590.37 feet; thence North 0 degrees 00 minutes 00 seconds, East, parallel to the West line of said Southeast Quarter, 143.91 feet to a point 10 feet South of the centerline of the industrial spur track to the True Point of Beginning; thence Easterly along a line 10 feet South of and parailel with said spur track, 301 feet, more or less; thence North 0 degrees 00 minutes 00 seconds, East, parallel to the West line of sald Southeast Quarter, 23 feet, more or less, to a point 25 feet South of the centerline of the main track of the Chicago Rock Island and Pacific Railroad Company; thence Westerly along a line 25 feet South of and parallel with said main track centerline 300 feet, more or less, thence South 0 degrees 00 minutes 00 seconds West, parallel to the West line of said Southeast Quarter, to the True Point of Beginning:

TO HAVE AND TO HOLD THE SAME, with all the rights, immunities, privileges, and appurtenances, thereto belonging, unto the safd Parties of the Second Part and unto their heirs and assigns forever; so that neither the said Party of the First part, nor any other person or persons, for it or in its name or behalf, shall or will hereafter claim or demand any right or title to the aforesaid premises or any part thereof, but they and each of them shall, by these presents, be excluded and forever barred.

IN WITNESS WHEREOF. The said Party of the First Part has caused these presents to be signed by its Vice-President and attested by its Secretary, and the corporate seal to be heret attached, the day and year first above written.

ATTEST:

(CORP SEAL)

SCF, INC.

Linda 5 . Moser

By F. Craig Lack

Secretary

STATE OF MISSOURI )

COUNTY OF BUCHANAN)

SS.

On this 13th day of February, 1984, before me, appeared F. Craig Lack, to me personally known, who being duly sworn, did say that he is the Vice-president of SCF., Inc., a corpration, and that the seal affixed to the foregoing instrument is the corporate seal of said corporation and that sald instrument was signed and sealed in behalf of said corporation by authority of its Board of Directors, and the said F. Craig Lack acknowledged said instrument to be the free act and depd of said corporation.

IN WITNESS WHEREOF, I have hereunto set my hand and affixed my notarial seal at my office in St. Joseph, Missouri, the day and year last above written.

(SEAL)

Stephen J. Briggs (Stephen J. Briggs) Notary Public with and for said County and

My Commission Expires: February 19, 1985

Filed for record the 21 day of February A.D. 1984 at 10:30 A.M.

\section{WARRANIY DEED}

Clarence Nold and Amanda Doris Nold, husband and wife, CONVEY AND WARRANT TO: Laipp1 Farms, Inse a Kansas Corporation

The North Half of the South Half of the Southwest

Quarter of Section Nine (9), Towaship Three (3),

Rage. Twenty-Two (22), and the South Half of the

Northyalf of the Southwest Quarter of Section

Nine (9) Township Three (3), Range Twenty-Two

(22), Doniphan Cotnty, Kansas,

for the consideration of ONE DOLLAR and ether valuable considerations.
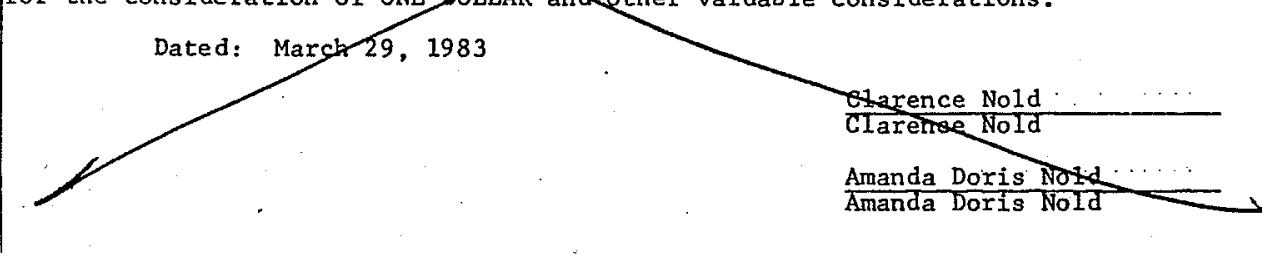
Court Documents Related to

Railroad Right-of-Way Abandonment of Easement 
IN THE DISTRICT COURT OF DONIRHAN COUNTY, KANSAS.

THE BOARD OF COUNTY COMMISSIONERS OF

DONIPHAN COUNTY, KANSAS,

vs.

LONNIE L. BEAR, ET AL., VS.

JAMES L. RUDDY, ET AL.,
PLAINTIFF, No. 91 C 26

DEFENDANTS, INTERVENOR-DEFENDANTS.

\section{ORDER NUNC PRO TUNC}

Now on this the $23^{\text {rd }}$ day of

March 1993, the above entitled matter comes on for hearing before the court on the motion of William R. McQuillan. The movant appears in person. There are no other appearances.

Thereupon, the motion is presented to the Court and the Court, after consideration thereof, FINDS that no notice of hearing upon the motion is required and that the same should be heard without notice instanter on this date and IT IS BY THE COURT SO ORDERED.

Thereupon, evidence is introduced upon the motion, and the Court, after consideration thereof, FINDS: 1. All allegations contained in the Motion are true.

2. On October 13, 1992, a Journal Entry of same date was entered in the above proceeding wherein the Court ordered and decreed that the individual Intervenor-Defendants 
Page 2 - Order Nunc Pro Tunc, Board of County Commissioners of Doniphan County, Kansas vs. Lonnie L. Bear, Et al, vs. James L. Ruddy, Et al, Case No. 91 C 26

were the owners of the fee simple title in and to certain tracts of reai estate. Specifically paragraphs 6 and 21 of said Journal Entry provided as follows, to wit:

"6. (a) As to that property referred to in the Petition as Exhibit 16 and described as all of the abandoned railroad right-of-way in the West 60 acres of the Southwest Quarter and middle 50 feet of right-of-way in the East 100 acres of the southwest Quarter less highway right-of-way all in Section 33, Township 3, Range 20 East and referred to by the Doniphan County Appraiser's office as parcel no. INO15A 1183303010 005.00 , except as to that part thereof in the West 60 acres described in paragraph 5 above, the south half of this former servient estate is located on real estate owned by intervenor-defendants, George H. Johnson, Jr. and Erma L. Johnson, by virtue of a deed recorded in Book 166 Page 81. By reason of abandonment of the easement premises the full fee simple title thereto should be quieted in intervenor-defendants, George H. Johnson, Jr. and Erma L. Johnson, and against defendant, Dirt and Grave1, Inc.

"(b) BY virtue of the provisions of subparagraph (a) above, the parcel number set forth therein should be stricken from the records in the Doniphan County Appraiser's Office and such parcel should be included in the parcel number of the former servient estate, number 1183303001010.00 .

"(c) The former servient estate lying on the North one-half of easement premises described in subparagraph (a) above is presently titled in fee simple in intervenor-defendant, Caudle Development Co., Inc., by virtue of a deed recorded in Book 171 Page 338. By reason of abandonment of the rallroad right-of-way the full fee simple title thereto should be quieted in intervenor-defendant, Caudle Development Co., Inc. and against the defendant, Dirt and Gravel, Inc. 
Page 3 - Order Nunc Pro Tunc, Board of County Commissioners of Doniphan County, Kansas vs. Lonnie L. Bear, Et al, vs. James L. Ruddy, Et al, Case No. 91 C 26

"(d) By virtue of the provisions of subparagraph (c) above, the above described parcel number should be stricken from the records in the Doniphan County Appraiser's office and such parcel should be included in the parcel number of the former servient estate, number $1183303001003.00 . "$

"21. (a) The property referred to in the Petition as Exhibit No. 43 and described as abandoned railroad right-of-way in the East Half of the Northeast Quarter and the southeast Quarter except the North 75 feet less highway right-of-way and referred to by the Doniphan County Appraiser's office as parcel no. 13203 01001005 and 1320304007001.00 and Denton $14 \mathrm{C}$, $14 \mathrm{C}-1,14 \mathrm{C}-2$ is located on the former servient estate of the City of Denton, Kansas and Atchison County Farmers Union Cooperative Association by virtue of deeds recorded in Book 166, Page 597 and 600 and the former servient estate of Roberts Bros. Inc., a Kansas Corporation by virtue of deed recorded in Book 159, Page 567. The North 74 feet exception above referred to consists of land deeded by the railroad's bankruptcy trustee to the said Roberts Bros. Inc. by Quit Claim Deed recorded in Book 171, Page 282. By reason of abandonment of the railroad right-of-way the dominant estate has been finally terminated and extinguished and, the full fee simple title to that portion of such tract lying south of the centerline of the former mainline railroad tracks should be quieted in intervenor-defendants, City of Denton and Atchison County Farmers Union Cooperative Association, and against the defendant, Dirt and Gravel, Inc.

"(b) By virtue of the provisions of subparagraph (a) above, the parcel number described therein should be stricken from the records in the Doniphan County Appraiser's office and that portion of such parcel lying south of the centerline of the former mainline railroad tracks should be included in the parcel numbers of the former servient estate, numbers 132030 $4003001.00,1320304007002.00$ and 1320304017 002.00 . 
Page 4 - Order Nunc Pro Tunc, Board of County Commissioners of Doniphan County, Kansas vs. Lonnie I. Bear, Et al, vs. James L. Ruddy, Et al, Case No. 91 C 26

"(c) The former servient estate lying North of the centerline of the former mainline railroad tracks of said easement premises described in subparagraph (a) above is presently titled in fee simple in intervenordefendant, Robert Bros. Inc, a Kansas Corporation, by virtue of deeds recorded in Book 171, Page 282 and Book 159, Page 567. By reason of abandonment of the railroad right-of-way the full fee simple title thereto should be quieted in intervenor-defendant, Robert Bros. Inc., a Kansas Corporation, and against the defendant, Dirt and Gravel, Inc.

"(d) By virtue of the provisions of subparagraph (c) above, the above described parcel number should be stricken from the records in the Doniphan County Appraiser's office and that portion of such parcel lying North of the centerline of the former mainline railroad tracks should be included in the parcel number of the former servient estate, number 132-030-10-01-1."

3. Due to clerical mistake the real

estate descriptions and related references set forth in said paragraphs 6 and 21 of the October 13, 1992, Journal Entry were erroneously set forth and pursuant to K.S.A. 60-260(a) an order Nunc Pro Tunc should enter herein correcting the said real estate descriptions and related references and that paragraphs 6 and 21 of the aforesaid Journal Entry dated October 13, 1992, should read as follows, to wit:

"6. (a) As to that property referred to in the Petition as Exhibit 16 and described as all of the abandoned railroad right-of-way in the West 60 acres of the southwest Quarter and middle 50 feet of right-of-way in the East 100 acres of the Southwest quarter less 
Page 5 - Order Nunc Pro Tunc, rard of County Commissioners of Doniphan County, Kansas vs. Lonnie L. Bear, Et al, vs. James L. Ruddy, Et al, Case No. 91 C 26

highway right-of-way all in Section 33, Township 3, Range 20 East and referred to by the Doniphan County Appraiser's Office as parcel no. INO15A 1183303010 005.00 , except as to that part thereof in the West 60 acres described in paragraph 5 above, the south half of this former servient estate is adjoining and located on real estate owned by intervenor-defendants, George $H$. Johnson, Jr. and Erma I. Johnson, by virtue of a deed recorded in Book 166 Page 81. By reason of abandonment of the easement premises the full fee simple title thereto should be quieted in intervenor-defendants, George H. Johnson, Jr. and Erma L. Johnson, and against defendant, Dirt and Gravel, Inc.

"(b) By virtue of the provisions of subparagraph (a) above, the parcel number set forth therein should be stricken from the records in the Doniphan County Appraiser's office and such parcel should be included in the parcel number of the former servient estate, number 1183303001010.00 .

"(c) The former servient estate lying on the North one-half of easement premises described in subparagraph (a) above is adjoining and located on land presently titled in fee simple in intervenor-defendant, Caudle Development Co., Inc., by virtue of a deed recorded in Book 171 Page 338. By reason of abandonment of the railroad right-of-way the full fee simple title thereto should be quieted in intervenor-defendant, Caudle Development Co., Inc. and against the defendant, Dirt and Gravel, Inc.

"(d) By virtue of the provisions of subparagraph (c) above, the above described parcel number should be stricken from the records in the Doniphan County Appraiser's office and such parcel should be included in the parcel number of the former servient estate, number 1183303001003.00 .

"(e) As to all of that property referred to in the Petition as Exhibit 16 and described as all of the abandoned railroad right-of-way in the West 60 acres of 
Page 6 - Order Nunc Pro Tunc, Board of County Commissioners of Doniphan County, Kansas vs. Lonnie L. Bear, Et al, vs. James L. Ruddy, Et al, Case No. 91 C 26

the Southwest Quarter and middle 50 feet of right-of--way in the East 100 acres of the southwest quarter less highway right-of-way all in section 33, Township 3, Range 20 East and referred to by the Doniphan County Appraiser's Office as parcel. No. INO15A 1183303010 005.00 , except as to that part referred to in paragraphs (a) - (d) above, such land is adjoining and located on the former servient estate of Paul $\mathrm{C}$. Johnson and Mary Linn Johnson by virtue of a deed recorded in Book 166 , Page 381 . By reason of the abandonment of the railroad right-of-way, the dominant estate has been finally terminated and extinguished and the full fee simple title to such real estate should be quieted in intervenordefendants, Paul C. Johnson and Mary Linn Johnson, and against defendant, Dirt and Gravel, Inc."

"21. (a) The property referred to in the Petition as Exhibit No. 43 and described as abandoned railroad right-of-way in the East Half of the Northeast Quarter and the Southeast Quarter except the North 75 feet less highway right-of-way and referred to by the Doniphan County Appraiser's Office as parcel no. 13203 01001005 and 1320304007001.00 and Denton $14 \mathrm{C}$, $14 \mathrm{C}-1,14 \mathrm{C}-2$ is located on the former servient estate of the City of Denton, Kansas and Atchison County Farmers Union Cooperative Association by virtue of deeds recorded in Book 166, Page 597 and 600 and the former servient estate of Roberts Farms, Inc., formerly Roberts Bros. Inc., a Kansas Corporation by virtue of deed recorded in Book 159, Page 567. The North 75 feet exception above referred to consists of land deeded by the railroad's bankruptcy trustee to the said Roberts Bros. Inc. by quit Claim Deed recorded in Book 171, Page 282. By reason of abandonment of the railroad right-of-way the dominant estate has been finally terminated and extinguished and, the full fee simple title to that part of the land described and referred to in the aforesaid Exhibit No. 43 which is described as follows:

Beginning at the Northwest Corner of the first tract of land described and conveyed to the 
Page 7 - Order Nunc Pro Tunc, Board of County Commissioners of Doniphan County, Kansas vs. Lonnie I. Bear, Et al, vs. James L. Ruddy, Et al, Case No. 91 C 26

Atchison County Farmers Union Cooperative Association by William H. Gibbons as Trustee for the Chicago, Rock Island and Pacific Railroad Company in that certain quit claim Deed recorded in the office of the Doniphan County Register of Deeds in Book 166 at Page 597, thence North on the East line of $F$ street, 138 feet, more or less, to a point on the South right-of-way line of North street, thence in a Northeasterly direction along the South right-of-way line of North street, 640 feet, more or less, to the West right-of-way line of $D$ street, thence south and East along the West right-of-way line of $D$ street, 150 feet, more or less, to the Northeast corner of the aforementioned first tract described in the deed recorded in Book 166, Page 597, thence Southwesterly along the North line of the aforementioned first tract 718 feet, more or less, to the point of beginning.

AND，

Commencing at a point which is the Northwest Corner of the second tract of land described and conveyed by the Atchison County Farmers Union Cooperative Association, a cooperative corporation to the City of Denton, Kansas in a deed duly recorded in the office of the Doniphan County Register of Deeds in Book 166 at Page 600, thence North along the East right-of-way line of $D$ street to the centerline of the old main track of the Chicago, Rock Island and Pacific Railroad Company (CRI\&P), thence Northeasterly 695 feet, more or less, along the centerline of the main track and parallel to the North line of the aforementioned second tract described in the deed recorded in Book 166, Page 600, a distance of 695 feet, more or less, thence southeasterly to a point which is the Northeast Corner of the aforementioned second 
Page 8 - order Nunc Pro Tunc, Board of County Commissioners of Doniphan County, Kansas vs. Lonnie L. Bear, Et al, vs. James L. Ruddy, Et al, Case No. 91 C 26

tract described in the deed recorded in Book 166, Page 600, thence Southwesterly along the North line of the aforementioned second tract described in the Deed recorded in Book 166, Page 600 to the point of beginning;

should be quieted in intervenor-defendants, City of Denton and Atchison County Farmers Union Cooperative Association, and against the defendant, Dirt and Gravel, Inc.

" (b) By virtue of the provisions of subparagraph (a) above, the parcel number described therein should be stricken from the records in the Doniphan County Appraiser's office and that portion of such parcel specifically described in subparagraph (a) last above should be included in the parcel numbers of the former servient estate, numbers 1320304003 $001.00,1320304007002.00$ and 1320304017002.00 .

"(c) As to that part of the former servient estate lying North of the centerline of the former mainline railroad track of said easement premises described in subparagraph (a) above which adjoins land presently titled in fee simple in intervenor-defendant, Roberts Farms, Inc., formerly Roberts Bros. Inc., a Kansas Corporation, by virtue of deeds recorded in Book 171, Page 282 and Book 159, Page 567, to wit:

Commencing at the southeast corner of a tract of land conveyed by William Gibbons as Trustee of the Chicago, Rock Island and Pacific Railroad Company (CRI\&P) to Roberts Brothers Inc. in a deed duly recorded in the office of the Doniphan County Register of Deeds in Book 171 at Page 282, thence South to the center line of the old main track of the CRI\&P, then Southwesterly along the center line of the main track 1,395 feet more or less, to a point, thence North to the Southwest corner of the aforementioned tract described in the deed 
Page 9 - Order Nunc Pro Tunc, Board of County Commissioners of Doniphan County, Kansas vs. Lonnie L. Bear, Et al, vs. James L. Ruddy, Et al, Case No. 91 C 26

recorded in Book 171, Fage 282, thence Northeasterly 1,395 feet more or less to the point of beginning,

by reason of the abandonment of the railroad right-ofway, the full fee simple title thereto should be quieted in intervenor-defendant, Roberts Farms, Inc., formerly Roberts Bros. Inc., a Kansas Corporation, and against the defendant, Dirt and Gravel, Inc.

"(d) By virtue of the provisions of subparagraph (c) above, the above described parcel number should be stricken from the records in the Doniphan County Appraiser's Office and that portion of such parcel specifically described in subparagraph (c) above should be included in the parcel number of the former servient estate, number 132-030-10-01-1."

IT IS THEREFORE BY THE COURT CONSIDERED, ORDERED AND ADJUDGED:

A. The findings hereinbefore made constitute and are a part of the judgment and decree herein entered.

B. That paragraphs 6 and 21 of the October 13, 1992, Journal Entry and the real estate set forth therein should read as follows, to wit:

"6. (a) As to that property referred to in the Petition as Exhibit 16 and described as all of the abandoned railroad right-of-way in the west 60 acres of the Southwest Quarter and middle 50 feet of right-of-way in the East 100 acres of the southwest quarter less highway right-of-way all in section 33, Township 3, Range 20 East and referred to by the Doniphan County 
Page 10 - Order Nunc Pro Tunc, Board of County Commissioners of Doniphan County, Kansas vs. Lonnie L. Bear, Et al, vs. James I. Ruddy, Et al, Case No. 91 C 26

Appraiser's office as parcel no. INol5A 1183303010 005.00 , except as to that part thereof in the West 60 acres described in paragraph 5 above, the south half of this former servient estate is adjoining and located on real estate owned by intervenor-defendants, George $H$. Johnson, Jr. and Erma I. Johnson, by virtue of a deed recorded in Book 166 Page 81. By reason of abandonment of the easement premises the full fee simple title thereto should be quieted in intervenor-defendants, George H. Johnson, Jr. and Erma L. Johnson, and against defendant, Dirt and Gravel, Inc. $\cdots$

By virtue of the provisions of subparagraph (a) above, the parcel number set forth therein should be stricken from the records in the Doniphan County Appraiser's office and such parcel should be included in the parcel number of the former servient estate, number 1183303001010.00 .

"(c) The former servient estate lying on the North one-half of easement premises described in subparagraph (a) above is adjoining and located on land presently titled in fee simple in intervenor-defendant, Caudle Development Co., Inc., by virtue of a deed recorded in Book 171 Page 338. By reason of abandonment of the railroad right-of-way the full fee simple title thereto should be quieted in intervenor-defendant, caudle Development Co., Inc. and against the defendant, Dirt and Gravel, Inc.

"(d) By virtue of the provisions of subparagraph (c) above, the above described parcel number should be stricken from the records in the Doniphan County Appraiser's office and such parcel should be included in the parcel number of the former servient estate, number 11833030 vl 003.00 .

"(e) As to all of that property referred to in the petition as Exhibit 16 and described as all of the abandoned railroad right-of-way in the West 60 acres of 
Page 11 - Order Nunc Pro Tunc, Board of County Commissioners of James L. Ruddy. Kt al, Ls. Lonnie L. Bear, Et al, vs.

the Southwest Quarter and middle 50 feet of right-of--way in the East 100 acres of the southwest quarter less highway right-of-ray all in section 33, Township 3, Range Appraiser's office to by the Doniphan County 005.00 , except as as parcel No. INO15A 1183303010 (a) - (d) above, such that referred to in paragraphs the former servient estate 1 s adjoining and located on Linn Johnson by virtue of Page 381. By reason of the aband recorded in Book 166, right-of-way, the dominant abandonment of the railroad terminated and extinguished estate has been finally extinguished and the full fee simple title defendants, Paul should be quieted in intervenoragainst defendant. Johnson and Mary Linn Johnson, and

"21. (a) The property referred to in the Petition as Exhibit. No. 43 and described as abandoned railroad right-of-way in the East Half of the Northeast Quarter and the southeast Quarter except the North 75 feet less highway right-of-way and referred to by the Doniphan County Appraiser's office as parcel no. 13203 $14 \mathrm{C}-1,14 \mathrm{C}-2$ and 1320304007001.00 and Denton 14C, the city of is located on the former servient estate of Union Cansas and Atchison County Farmers in Bociation by virtue of deeds recorded estate 166, Page 597 and 600 and the former servient Inc Inc." a Kansas Corporation by virtue of deed recorded in Book 159, Page 567. The North 75 feet exception above referred to consists of land deeded by the railroad's bankruptcy. trustee to the said Roberts Bros. Inc. by Quit Claim Deed recorded in Book 171, Page 282. By reason of abandonment of the railroad right-of-way the dominant estate has been finally terminated and extinguished and, the full fee simp?a titls to that part of the land described and referred to in the aforesaid Exhibit No. 43 which is described as follows: 
Page 12 - Order Nunc Pro Tunc, Board of County Commissioners of Doniphan County, Kansas vs. Lonnie L. Bear, Et al, vs. James L. Ruddy, Et al, Case No. 91 C 26

Beginning at the Northwest Corner of the first tract of land described and conveyed to the Atchison County Farmers Union Cooperative Association by William H. Gibbons as Trustee for the Chicago, Rock Island and Pacific Railroad Company in that certain quit claim Deed recorded in the office of the Doniphan County Register of Deeds in Book 166 at Page 597, thence North on the East line of $F$ Street, 138 feet, more or less, to a point on the South right-of-way line of North street, thence in a Northeasterly direction along the South right-of-way line of North Street, 640 feet; more or less, to the West right-of-way line of $D$ street, thence south and East along the West right-of-way line of D street, 150 feet, more or less, to the Northeast corner of the aforementioned first tract described in the deed recorded in Book 166, Page 597, thence. Southwesterly along the North line of the aforementioned first tract 718 feet, more or less, to the point of beginning.

AND，

Commencing at a point which is the Northwest corner of the second tract of land described and conveyed by the Atchison County Farmers Union Cooperative Association, a cooperative corporation to the City of Denton, Kansas in a deed duly recorded in the office of the Doniphan County Register of Deeds in Book 166 at Page 600, thence North along the East right-of-way line of $D$ street to the centerline of the old main track of the Chicago, Rock Island and Pacific Railroad Company (CRI\&P), thence Northeasterly 695 feet, more or less, along the centerline of the main track and parallel to the North line of the aforementioned second tract described in the deed recorded in Book 166, Page 600 , a distance of 695 feet, more or less, thence Southeasterly to a point which is the Northeast 
Page 13 - Order Nunc Pro Tunc, Board of County Commissioners of Doniphan County, Kansas vs. Lonnie L. Bear, Et al, vs. James I. Ruddy, Et a1, Case No. 91 C 26

Corner of the aforementioned second tract described in the deed recorded in Book 166 , Page 600, thence Southwesterly along the North line of the aforementioned second tract described in the Deed recorded in Book ibo, Page 600 to the point of beginning;

should be quieted in intervenor-defendants, City of Denton and Atchison County Farmers Union Cooperative Association, and against the defendant, Dirt and Gravel, Inc.

""(b) By virtue of the provisions of subparagraph (a) above, the parcel number described therein should be stricken from the records in the Doniphan County Appraiser's office and that portion of such parcel specifically described in subparagraph (a) last above should be included in the parcel numbers of the former servient estate, numbers 1320304003 $001.00,1320304007002.00$ and 1320304017002.00 .

"(c) As to that part of the former servient estate lying North of the centerline of the former mainline railroad track of said easement premises described in subparagraph (a) above which adjoins land presently titled in fee simple in intervenor-defendant, Roberts Farms, Inc., formerly Roberts Bros. Inc., a Kansas Corporation, by virtue of deeds recorded in Book 171, Page 282 and Book 159, Page 567, to wit:

Commencing at the southeast corner of a tract of land conveyed by William Gibbons as Trustee of the Chicago, Rock Island and Pacific Railroad Company (CRI\&P) to Roberts Brothers Inc. in a deed duly recorded in the office of the Doniphan County Register of Deeds in Book 171 at Page 282 , thence south to the center line of the old main track of the CRI\&P, then Southwesterly along the center line of the main track 1,395 feet more or less, to a point, thence North to the southwest corner of the aforementioned tract described in the deed 
Page 14 - Order Nunc Pro Tunc, Board of County Commissioners of Doniphan County, Kansas vs. Lonnie L. Bear, Et al, vs. James L. RuddY, Et al, Case No. 91 C 26

recorded in Book 171, Page 282, thence Northeasterly 1,395 feet more or less to the point of beginning,

by reason of the abandonment of the railroad right-ofway, the full fee simple title thereto should be quieted in intervenor-defendant, Roberts Farms, Inc., formerly Roberts Bros. Inc., a Kansas Corporation, and against the defendant, Dirt and Gravel, Inc.

"(d) By virtue of the provisions of subparagraph (c) above, the above described parcel number should be stricken from the records in the Doniphan County Appraiser's office and that portion of such parcel specifically described in subparagraph (c) above should be included in the parcel number of the former servient estate, number 132-030-10-01-1."

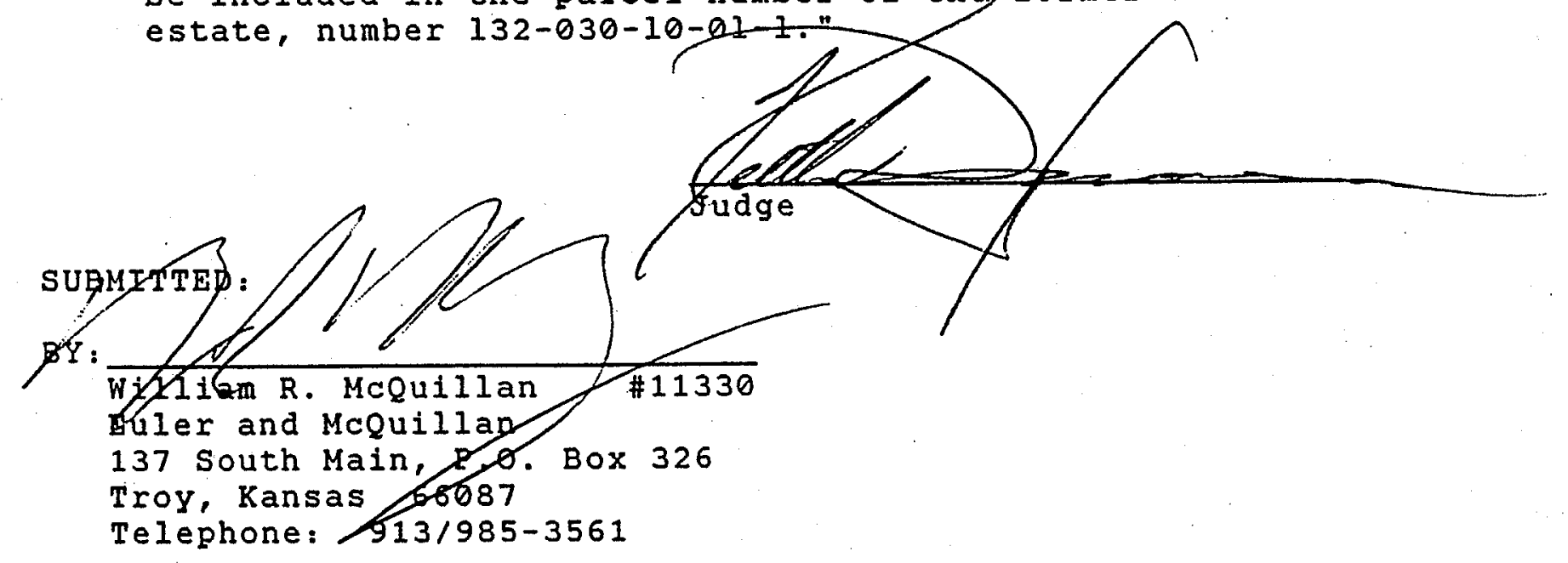


Articles of Incorporation and Dissolution for Bendena Grain Company, Inc. 
PQ JUL 16 AM $7: 55$ FULO
JACKHY GWER
SECRETAFY STATE
KANSAS

\section{ARTICLES OF INCORPORATION}

으

BENDENA GRAIN COMPANY

Pursuant to the provisions of

the Kansas General Corporation Code, the undersigned do hereby organize and incorporate a Kansas Corporation as follows: FIRST: The name of the corporation

is: BENDENA GRAIN COMPANY.

SECOND: The address of the

corporation's registered office in Kansas is: Highway 36 East, Wathena, Doniphan County, Kansas 66090.

THIRD: The name of the corporation $s$ Resident Agent at the above address is: Edwin Dennis Ford. FOURTH: This corporation is organized FOR PROFIT and the nature of the business to be conducted, and the purposes of the corporation, is to engage in any lawful act or activity for which corporations may be organized under the Kansas General corporation Code as such statement of purposes is contemplated and intended in K.S.A. Supp. 17-6002(3) specifically including, but in no way limited to, the agricultural and horticultural business, including but not limited to, the producing, planting, raising, harvesting or gathering of wheat, corn, grain sorghums, barley, oats, rye and potatoes and the milking of cows for dairy purposes, all as intented and contemplated in K.S. 1.1 . 1-5901.

FIFTH: The corporation is

authorized to issue only one class of stock, namely, Common stock. The total number of shares of common stock which the corporation shall have authority to issue is Five Hundred Thousand $(500,000)$ shares and the par value of each of such shares shall be One Dollar $(\$ 1.00)$.

\section{SIXTH: The name and mailing}

addresses of the incorporators are: 


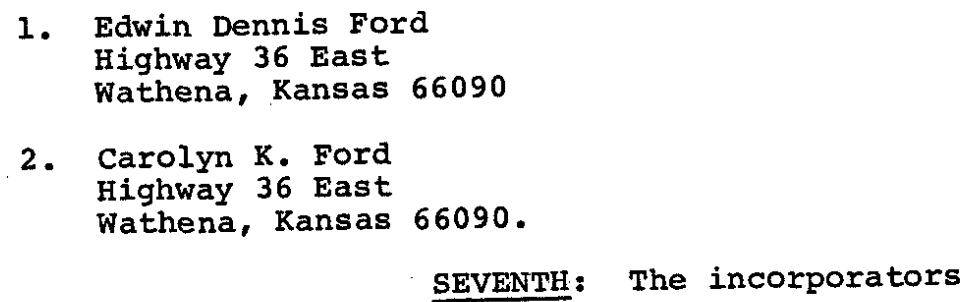

shall adopt the original by-laws of the corporation and shall elect the initial Board of Directors and perform such other acts as are authorized and provided in K.S.A. 17-6007 and 6008; thereafter, the Board of Directors shall be authorized to make, alter or repeal the by-laws of the corporation all as authorized in K.S.A. Supp. 17-6009 subject, however, to the right of the stockholders to likewise make, alter or repeal such by-laws.

IN WITNESS WHEREOF, the undexsigned incorporators have hereunto set their hand on this the 8 th day of JuIy; 1980

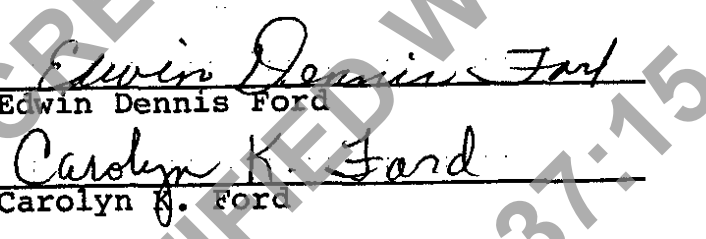

STATE OF KANSAS, COUNTYOF DONIPHAN, SS:

The foregoing instrument was acknowledged before me on this the 8 th day of July, 1980, by Edwin Dennis Ford and Carolyn Ko Ford.
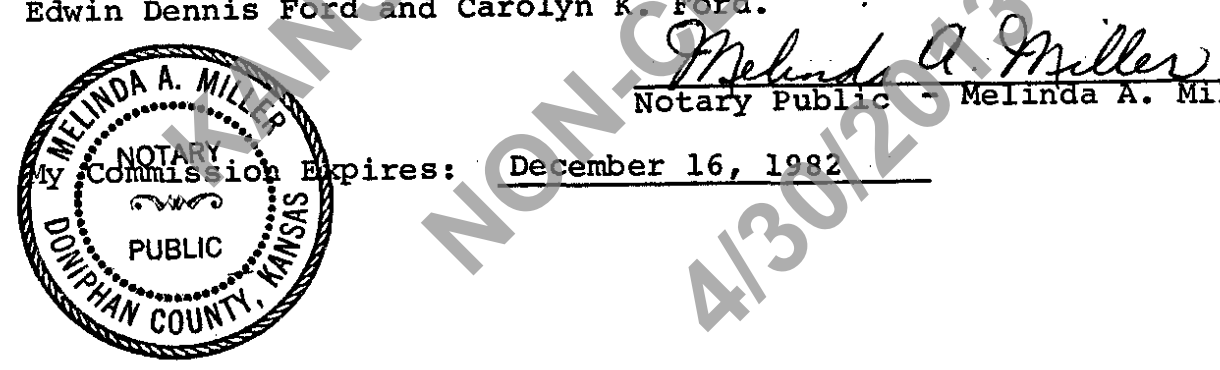
Notary Public - MeIinda A. Miller

December 16,1982 


\section{Contact Information}

Kansas Secretary of State

Ron Thornburgh

Memorial Hall, lst Floor

120 S.W. 10th Avenue

Topeka, KS 66612-1594

(785) 296-4564

kssos@kssos.org

www.kssos.org
Name of the corporation:

\section{Bendena Grain Company}

Name must match the name on record with the secretary of stme

All the stockholders with voting power do hereby consent to the dissolution of the corporation.

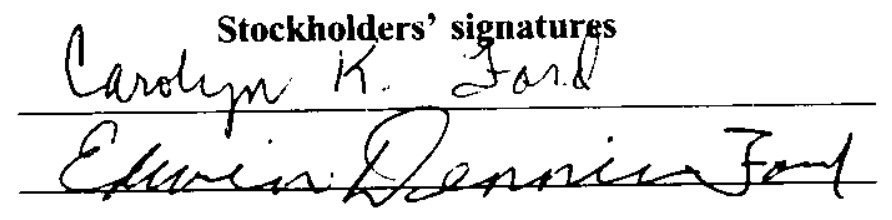

KANSAS SECRETARY OF STATE For Profit Corporation Dissolution by Written Consent

DW

53-01

All information must be completed or this document will not be accepted for filing.

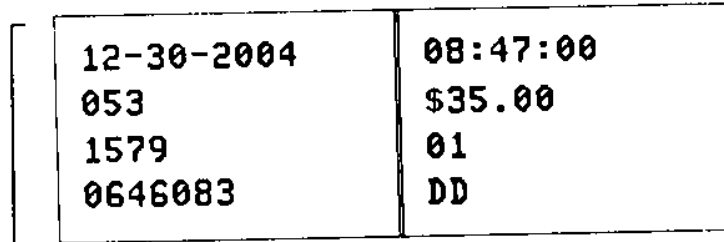

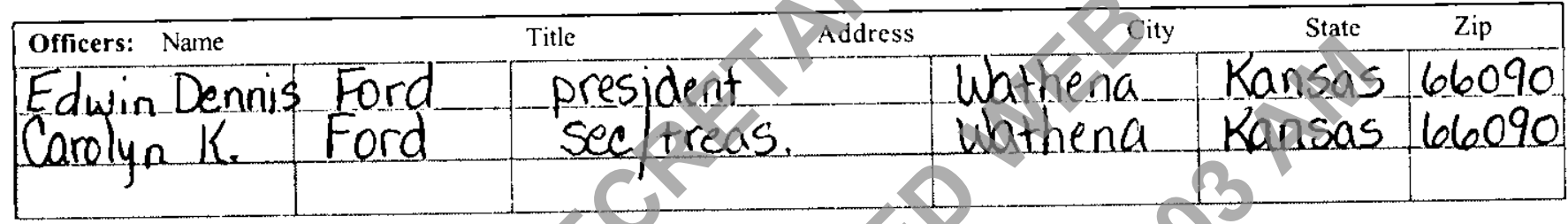

\begin{tabular}{|l|l|l|l|l|}
\hline Directors: Name & City O State & \\
\hline Same as above & & & & \\
\hline & & & & \\
\hline
\end{tabular}

1. Edwin Dennis Ford declare under penalty of perjury under the laws of the state of Kansas, that I am an officer of the above-named compration. that the above consent has been signed by or on behalf of ALL. stockholders entitled to vote on the dissolution, and that the foregoing is true and correct.

Executed on the $\frac{20}{\text { Day }} \frac{\mathrm{DeC}}{\text { Menth }} \cdot \frac{2004}{\text { Yeit }}$

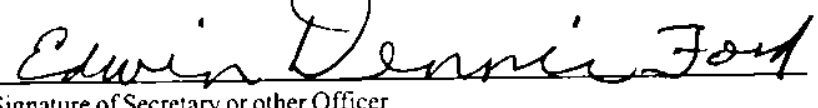

Signature of Secretary or other Officer

\section{Instructions}

1. Submit this form in duplicate with the $\$ 35$ filing fee.

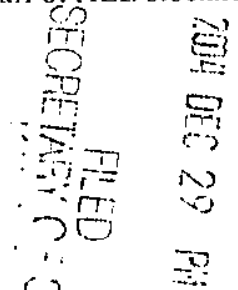

2. If this form is submitted after the close of the corporation's tax year, an annual report and franchise tax must be submitted for that year.

Notice: There is a $\$ 25$ service fee for all returned checks. 
Argonne

Environmental Science Division

Argonne National Laboratory

9700 South Cass Avenue, BIdg. 203

Argonne, IL 60439-4843

www.anl.gov 